\title{
The role of biomarkers in preclinical and prodromal Alzheimer's disease
}

Citation for published version (APA):

Vos, S. J. B. (2014). The role of biomarkers in preclinical and prodromal Alzheimer's disease. [Doctoral Thesis, Maastricht University]. Uitgeverij BOXPress. https://doi.org/10.26481/dis.20140220sv

Document status and date:

Published: 01/01/2014

DOI:

10.26481/dis.20140220sv

Document Version:

Publisher's PDF, also known as Version of record

\section{Please check the document version of this publication:}

- A submitted manuscript is the version of the article upon submission and before peer-review. There can be important differences between the submitted version and the official published version of record.

People interested in the research are advised to contact the author for the final version of the publication, or visit the DOI to the publisher's website.

- The final author version and the galley proof are versions of the publication after peer review.

- The final published version features the final layout of the paper including the volume, issue and page numbers.

Link to publication

\footnotetext{
General rights rights.

- You may freely distribute the URL identifying the publication in the public portal. please follow below link for the End User Agreement:

www.umlib.nl/taverne-license

Take down policy

If you believe that this document breaches copyright please contact us at:

repository@maastrichtuniversity.nl

providing details and we will investigate your claim.
}

Copyright and moral rights for the publications made accessible in the public portal are retained by the authors and/or other copyright owners and it is a condition of accessing publications that users recognise and abide by the legal requirements associated with these

- Users may download and print one copy of any publication from the public portal for the purpose of private study or research.

- You may not further distribute the material or use it for any profit-making activity or commercial gain

If the publication is distributed under the terms of Article $25 \mathrm{fa}$ of the Dutch Copyright Act, indicated by the "Taverne" license above, 


\section{THE ROLE OF BIOMARKERS IN}

PRECLINICAL AND PRODROMAL ALZHEIMER'S DISEASE 
(C).J.B. Vos, Maastricht, 2014

Cover design: Karolien Pauly

Lay out: Stephanie Vos

Printed by: Proefschriftmaken.nl || Uitgeverij BOXPress

Published by: Uitgeverij BOXPress, 's-Hertogenbosch

ISBN: 978-90-8891-806-3 


\section{THE ROLE OF BIOMARKERS IN \\ PRECLINICAL AND PRODROMAL ALZHEIMER'S DISEASE}

PROEFSCHRIFT

Ter verkrijging van de graad van doctor aan de Universiteit Maastricht, op gezag van Rector Magnificius, Prof. dr. L.L.G. Soete, volgens het besluit van het college van Decanen, in het openbaar te verdedigen op donderdag 20 februari 2014 om 16:00 uur

door

Stephanie Jo Berthe Vos

Geboren op 30 juli 1986 te Tongeren (België) 


\section{Promotor:}

Prof. dr. F.R.J. Verhey

\section{Co-promotor:}

Dr. P.J. Visser

\section{Beoordelingscommissie:}

Prof. dr. R.W.H.M. Ponds (voorzitter)

Prof. dr. F. Jessen (University Hospital Bonn, Duitsland)

Dr. S. Köhler

Prof. dr. R.J. van Oostenbrugge

Dr. N.S. Schoonenboom (Kennemer Gasthuis, Haarlem)

The research described in this thesis was performed at the Department of Psychiatry and Neuropsychology, School for Mental Health and Neuroscience, Maastricht University, Alzheimer Center Limburg, Maastricht, the Netherlands; and Department of Neurology, The Knight Alzheimer's Disease Research Center, Washington University School of Medicine, St. Louis, Missouri, USA.

The research presented in this thesis was supported by the Center for Translational Molecular Medicine (www.ctmm.nl) project LeARN (grant 02N-01).

Printing of this thesis was kindly supported by Alzheimer Nederland, Internationale Stichting Alzheimer Onderzoek, Nutricia Advanced Medical Nutrition, Lundbeck, and CTMM LeARN.
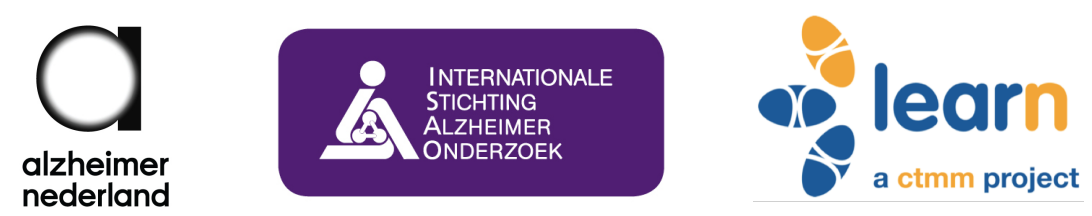


\section{Voor oma}


"In some ways disease does not exist until we have agreed that it does - by perceiving, naming, and responding to it."

Charles E. Rosenberg 


\section{CONTENTS}

CHAPTER 1 General introduction, aims and outline thesis

PART I BIOMARKERS FOR AD-TYPE DEMENTIA IN SUBJECTS WITH MCI

CHAPTER 2 Biomarkers as predictor for conversion from mild cognitive

impairment to Alzheimer-type dementia. Implications for trial design

Journal of Alzheimer's disease 2010

CHAPTER 3 Test sequence of CSF and MRI biomarkers for prediction of AD

in subjects with MCI

Neurobiology of Aging 2012

CHAPTER 4 Prediction of Alzheimer's disease in subjects with amnestic and

non-amnestic MCI

Neurology 2013

CHAPTER 5 Injury markers predict time to dementia in subjects with MCI and amyloid pathology

Neurology 2012

CHAPTER 6 Variability of CSF Alzheimer's disease biomarkers:

Implications for clinical practice

\section{PART II DIAGNOSTIC CRITERIA FOR PREDEMENTIA ALZHEIMER'S DISEASE}

CHAPTER 7 Comparison of International Working Group criteria and National

Institute on Aging-Alzheimer's Association criteria for

Alzheimer's disease

Alzheimer's \& Dementia 2012

CHAPTER 8 Preclinical Alzheimer's disease and its outcome:

a longitudinal cohort study

The Lancet Neurology 2013

CHAPTER 9 Prevalence and prognosis of prodromal Alzheimer's disease:

comparison of International Working Group criteria and National Institute on Aging-Alzheimer's Association criteria 
CHAPTER 10 General discussion

Nederlandse samenvatting 191

Author affiliations 195

$\begin{array}{ll}\text { Publications } & 201\end{array}$

Author information 203

Acknowledgements / dankwoord 205 


\section{CHAPTER 1}

GENERAL INTRODUCTION

AIM

OUTLINE OF THESIS 


\section{GENERAL INTRODUCTION}

\section{Alzheimer's disease}

Alzheimer's disease (AD) is a progressive neurodegenerative disease consisting of a long asymptomatic stage, a stage of mild cognitive impairment and finally a dementia stage in which impaired cognition leads to interference in daily life activities. AD accounts for the majority of dementia cases among people over the age of $65^{1,2}$ and is worldwide one of the fastest growing threats to health with no cure available so far. ${ }^{3}$ The global prevalence of dementia is now 35.6 million $^{4}$ and is expected to affect more than 80 million people worldwide by $2040.5,6$ The cost of dementia care is likely to double by that time. ${ }^{7}$ Advances in early diagnosis of $\mathrm{AD}$ as well as in therapy are needed to anticipate and manage future societal burden and costs. ${ }^{2,8}$

According to the original criteria for AD that were published in 1984, ${ }^{9}$ a clinical diagnosis was only possible when someone is already demented. At that stage extensive neuropathological damage has occurred and treatment has limited effects. ${ }^{10}$ The last decade, tremendous progress has been made in the area of biomarkers for AD neuropathology. This makes it possible to identify subjects with AD before they are demented, which opens new opportunities for treatment and prevention of the disease. ${ }^{11}$ At present, one of the major challenges is to investigate how biomarkers or biomarker combinations can be used for diagnosis and prognosis of AD for clinical and research purposes. In this thesis we will study the use of $\mathrm{AD}$ biomarkers in the stage before dementia, i.e. the predementia stages.

\section{Biomarkers for Alzheimer's disease neuropathology}

The neuropathological hallmarks of AD include extracellular amyloid plaques, composed of aggregated amyloid- $\beta$, and intracellular neurofibrillary tangles, consisting of hyperphosphorylated tau proteins.12,13 Other important pathological features are neuroinflammation and neurodegeneration, which is characterized by synaptic loss, neuron loss, and overall brain atrophy. ${ }^{14}$ AD biomarkers should be surrogate markers of these neuropathological changes. ${ }^{15}$ We can differentiate between biomarkers for amyloid pathology and biomarkers of neuronal injury. Previous studies have shown that decreased amyloid- $\beta$ in cerebrospinal fluid (CSF) ${ }^{16-18}$ and increased binding of positron emission tomography (PET)-amyloid tracers ${ }^{19,20}$ are good markers of amyloid pathology, whereas increased total tau and phosphorylated tau in CSF, ${ }^{16-18}$ hippocampal atrophy on magnetic resonance imaging (MRI) ${ }^{12,21,22}$ and hypometabolism as measured by PET $^{23,24}$ have shown to be good markers of neuronal injury.

\section{Diagnostic criteria for Alzheimer's disease}

Advances in the AD biomarker field have changed our view on AD. This, in turn, has recently led to the development of new diagnostic criteria for AD. ${ }^{25-29}$ Currently, these criteria are considered research criteria although they will likely be applied in clinical practice in the 
future. Below we describe the old and new classification of $\mathrm{AD}$ and the role of biomarkers in the new criteria.

\section{Old classification}

According to the original criteria for $\mathrm{AD},{ }^{9}$ a clinical diagnosis was primarily based on clinical symptoms and was only possible when clinical symptoms surpass the threshold of dementia. These criteria for AD-type dementia required cognitive impairments in memory and at least one other domain that lead to interference in daily living.

Subjects with cognitive impairments that are still able to function independently in daily life were diagnosed with mild cognitive impairment (MCI). ${ }^{30}$ These subjects are at increased risk of developing dementia, with annual progression rates from MCI to a clinical diagnosis of AD-type dementia of 5-15\%. ${ }^{31,32}$ Subjects with amnestic MCI are most likely to progress to AD-type dementia, although $\mathrm{AD}$ may also initially present as non-amnestic MCI. ${ }^{33-37}$ Several subjects with MCI will however remain stable or revert back to normal cognition. ${ }^{38}$ The challenge is to identify those subjects that will develop AD-type dementia as early as possible. Table 1 summarizes these stages of AD.

Table 1. Glossary of AD concepts according to the old and new classification

\begin{tabular}{|c|c|}
\hline OLD CLASSICIATION & \\
\hline Cognitively healthy & No cognitive impairments regardless of underlying AD pathology \\
\hline Amnestic MCI & $\begin{array}{l}\text { Impairments in memory (and possibly other domains) regardless of } \\
\text { underlying AD pathology }\end{array}$ \\
\hline Non-amnestic MCI & $\begin{array}{l}\text { Impairments in non-memory domain(s) regardless of underlying } \\
\text { AD pathology }\end{array}$ \\
\hline AD-type dementia & $\begin{array}{l}\text { Clinical diagnosis of AD-type dementia regardless of underlying } \\
\text { pathology }\end{array}$ \\
\hline NEW CLASSIFICATION & \\
\hline Preclinical AD / asymptomatic AD & Cognitively healthy with underlying AD pathology \\
\hline Prodromal AD / MCI due to AD & Mild cognitive impairment(s) with underlying AD pathology \\
\hline Dementia due to AD & $\begin{array}{l}\text { Clinical diagnosis of AD-type dementia with underlying AD } \\
\text { pathology }\end{array}$ \\
\hline
\end{tabular}

$\mathrm{AD}=$ Alzheimer's disease, $\mathrm{MCI}=$ mild cognitive impairment

\section{New classification}

The key differences of the new $\mathrm{AD}$ criteria from the original criteria are the incorporation of biomarkers and the distinction between different stages of the disease, which allows a diagnosis of AD before the dementia stage, i.e. in the predementia stages.

Alzheimer's disease is now subdivided in different clinical stages depending on cognitive status. Preclinical or asymptomatic $\mathrm{AD}$ is the earliest stage in which $\mathrm{AD}$ neuropathology begins to accumulate but cognitive performance is still normal. Prodromal $\mathrm{AD}$ or $\mathrm{MCI}$ due to $\mathrm{AD}$ is the next disease stage in which individuals experience $\mathrm{MCI}$. Dementia due to AD is the last stage with AD pathology and a clinical diagnosis of AD-type 
dementia. Table 1 summarizes these stages of $\mathrm{AD}$ and Figure 1 shows how these stages relate to the old classification.

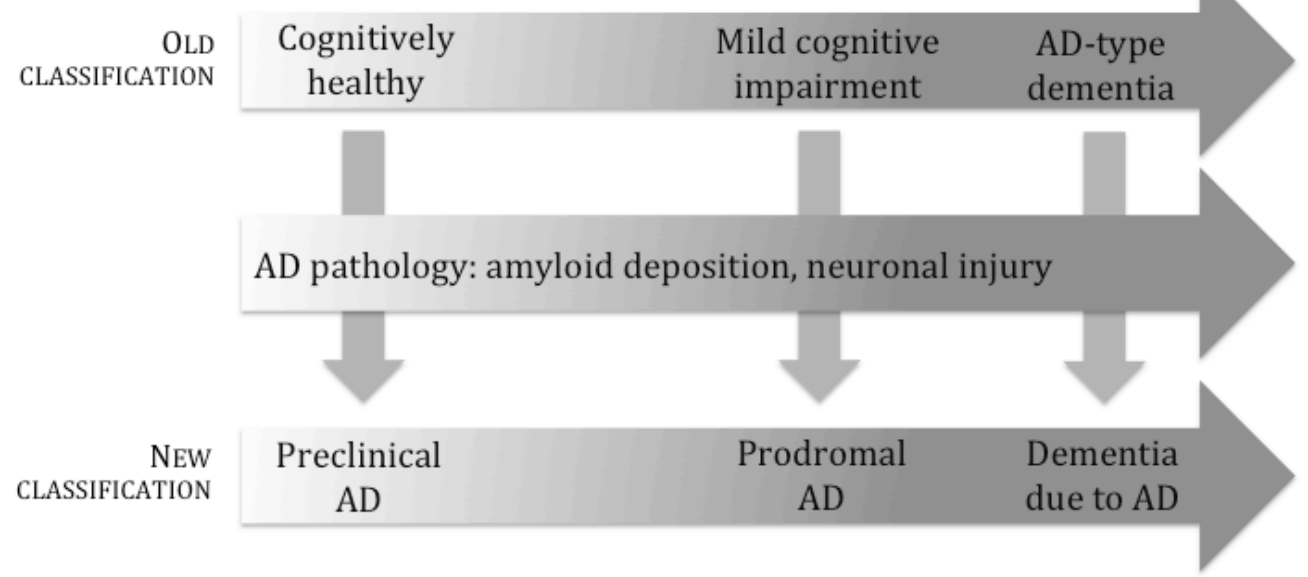

Figure 1. The relation between several AD stages of the old and new classification.

The international working group (IWG) of Dubois and colleagues was the first to present revised AD criteria. ${ }^{25,26}$ The IWG referred to subjects with normal cognition and amyloid pathology as asymptomatic at risk for AD. Furthermore, they introduced the concept of prodromal $\mathrm{AD}$, defined by at least one abnormal well-established $\mathrm{AD}$ biomarker in the presence of episodic memory impairment without interference in daily functioning. In presence of a clinical diagnosis of AD-type dementia, this latter group is referred to as $\mathrm{AD}$ dementia. While memory impairment is highlighted in the IWG criteria, other clinical phenotypes may be associated with atypical forms of AD. Later on, the National Institute on Aging and Alzheimer's Association (NIA-AA) workgroup proposed new revised criteria for AD. ${ }^{39}$ These criteria divide the preclinical AD stage in 3 ordered substages: abnormal amyloid markers (stage 1), abnormal amyloid and neuronal injury markers (stage 2) and abnormal amyloid and neuronal injury markers and subtle cognitive changes (stage 3). ${ }^{27}$ For the diagnosis of $\mathrm{MCI}$ due to $\mathrm{AD}$ or dementia due to $\mathrm{AD}$, the number of positive biomarkers are supposed to be related to the likelihood that the diagnosis is due to AD: both a positive amyloid and injury marker is high likelihood, one positive marker is intermediate likelihood, and both a negative amyloid and injury marker is unlikely that the cognitive/clinical impairments are due to AD. ${ }^{28,29}$ Conflicting amyloid and injury marker results are considered uninformative. The NIA-AA criteria emphasize that subjects can also present initially with non-memory problems. Before implementation of these proposed sets of criteria in clinical routine, the criteria need to be thoroughly validated to see whether they refer to a solid disease construct and whether they reflect different stages of disease severity with different progression rates to AD-type dementia. Figure 2 summarizes the proposed criteria for subjects with predementia AD. 


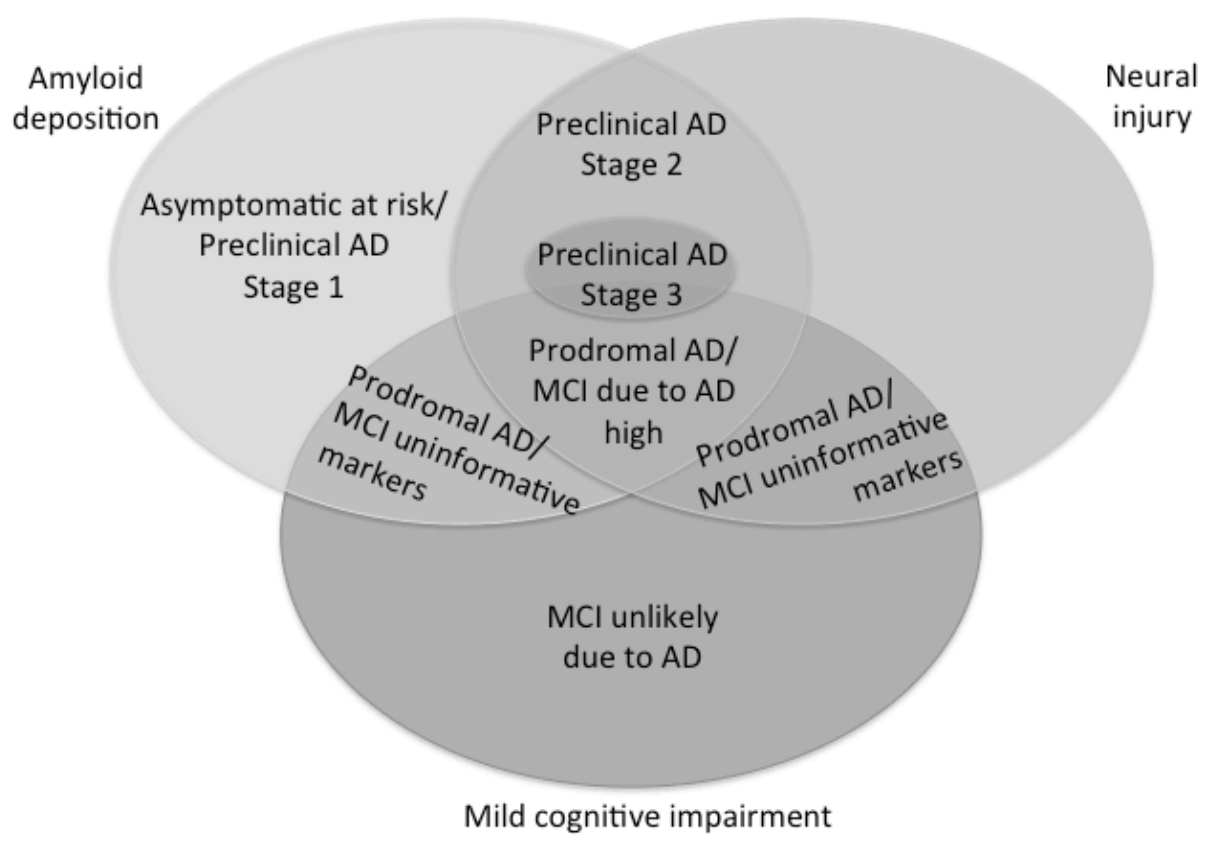

Figure 2. Summary of the proposed IWG and NIA-AA criteria for subjects with predementia AD

\section{Use of biomarkers in the new diagnostic criteria}

Appropriate biomarkers are needed to apply the recently proposed diagnostic criteria for AD. The last decades, $A D$ research has been dominated by the amyloid cascade hypothesis. ${ }^{40,41}$ This hypothesis states that AD pathophysiology starts with amyloid deposition 10 to 15 years before clinical symptoms appear, which in turn initiates multiple downstream events leading to neuronal injury and eventually to clinical symptoms. Based on the position in the amyloid cascade, amyloid markers are considered early AD markers, while neuronal injury markers may better predict future cognitive decline, since they become abnormal at a later stage, closer to clinical symptoms. ${ }^{42,43}$

As biomarkers are starting to find their way into clinical practice, knowledge about how they should be applied in clinical settings is urgently needed. It is not yet clear which biomarker or which combination of biomarkers we should use for optimal prediction of ADtype dementia at different stages of the disease, since the exact temporal order of $A D$ biomarker abnormality is not known yet. Moreover, it is difficult to understand the relative importance of different biomarkers when used together and to interpret biomarker results when they are conflicting. ${ }^{28}$ These issues need to be addressed before biomarkers can be widely applied in clinical practice.

There are also some problems with the CSF analysis itself. Previous studies showed considerable variability between analyses in the same laboratory as well as in different laboratories. ${ }^{44-48}$ The impact of CSF variability on the diagnostic CSF-based AD classification of subjects is not examined yet. Together with the lack of standardized CSF cut-offs to define markers as normal vs. abnormal, ${ }^{49}$ this may have a major influence on diagnosis or treatment decisions in clinical practice. 


\section{AIM OF THE THESIS}

The general aim of this thesis was to investigate the role of biomarkers for diagnosis and prognosis of Alzheimer's disease in cognitively normal subjects, subjects with amnestic MCI, and subjects with non-amnestic MCI.

Our study addressed the following questions:

[1] What is the predictive ability of CSF biomarkers for AD-type dementia in subjects with MCI?

[2] What is the best combination of CSF and MRI biomarkers for predicting AD-type dementia in subjects with MCI?

[3] Is the predictive ability of biomarkers for AD-type dementia different in subjects with amnestic and non-amnestic MCI?

[4] Which biomarkers can predict clinical progression in subjects with abnormal amyloid- $ß$ in CSF?

[5] What is the influence of CSF variability within and between centers on CSF-based AD diagnosis?

[6] What is the prevalence and prognosis of preclinical AD based on the NIA-AA criteria?

[7] What is the prevalence and prognosis of prodromal AD based on the IWG and NIAAA criteria?

\section{OUTLINE OF THE THESIS}

The thesis consists of two parts. In part I, we describe the diagnostic and prognostic ability of single and combined AD biomarkers in subjects with MCI. In part II, we focus on the recently proposed diagnostic criteria for preclinical and prodromal AD. These criteria highlight the use of biomarkers to increase the certainty of whether a subject has underlying AD pathology or not.

The outline is as follows:

\section{PART I}

Chapter 2 provides a review and meta-analysis of the use of CSF and plasma AD biomarkers for the prediction of AD-type dementia in subjects with MCI. 
Chapter 3 compares the predictive ability of CSF and MRI biomarkers for AD-type dementia in subjects with MCI of the European multi-center memory clinic-based DESCRIPA study. We determined the optimal test sequence of these biomarkers.

In Chapter 4 the predictive ability of CSF markers, MRI markers and APOE genotype for ADtype dementia is compared in subjects with amnestic and non-amnestic MCI of the DESCRIPA study.

Chapter 5 assesses the progression rate to dementia and identifies prognostic markers in subjects with MCI and evidence of CSF amyloid pathology recruited from the DESCRIPA study.

In Chapter 6 we report the variability between CSF analyses in the same laboratory and in different laboratories and the influence of this variability on CSF-based AD diagnosis in a memory-clinic population of the Dutch multi-center LeARN study.

\section{$\underline{\text { PART II }}$}

In Chapter 7 we compare the theoretical framework of the International Working Group (IWG) criteria and National Institute on Aging-Alzheimer's Association (NIA-AA) criteria for Alzheimer's disease

Chapter 8 describes the prevalence and prognosis of subjects with preclinical AD according to CSF biomarkers and the proposed NIA-AA criteria. Subjects were recruited from longitudinal studies of memory and aging in cognitively normal community-dwelling volunteers at the Knight Alzheimer's Disease Research Center (ADRC) of the Washington University School of Medicine in St. Louis (USA).

In Chapter 9 the prevalence and prognosis of prodromal AD is examined based on wellestablished biomarkers and the proposed NIA-AA and IWG criteria. Subjects were recruited from multiple centers of the European Alzheimer's Disease Consortium (EADC) and the Alzheimer's Disease Neuroimaging Initiative (ADNI).

Last, Chapter 10 provides a general discussion, summary of the main findings, list of author affiliations, list of publications, information about the author, and acknowledgements. 


\section{REFERENCES}

1. Ferri CP, Prince M, Brayne C, et al. Global prevalence of dementia: a Delphi consensus study. Lancet 2005;366:2112-2117.

2. Hampel H, Prvulovic D, Teipel S, et al. The future of Alzheimer's disease: the next 10 years. Progress in neurobiology 2011;95:718-728.

3. Mangialasche F, Solomon A, Winblad B, Mecocci P, Kivipelto M. Alzheimer's disease: clinical trials and drug development. Lancet neurology 2010;9:702-716.

4. Prince M, Bryce R, Albanese E, Wimo A, Ribeiro W, Ferri CP. The global prevalence of dementia: A systematic review and metaanalysis. Alzheimers \& Dementia 2013;9:63-75.

5. Jones RW, McCrone P, Guilhaume C. Cost effectiveness of memantine in Alzheimer's disease: an analysis based on a probabilistic Markov model from a UK perspective. Drugs \& aging 2004;21:607620.

6. Reitz C, Brayne C, Mayeux R. Epidemiology of Alzheimer disease. Nature reviews Neurology 2011;7:137-152.

7. Hurd MD, Martorell P, Langa KM. Monetary costs of dementia in the United States. The New England journal of medicine 2013;369:489-490.

8. Wimo A, Jonsson L, Bond J, Prince M, Winblad B. The worldwide economic impact of dementia 2010. Alzheimer's \& dementia : the journal of the Alzheimer's Association 2013;9:1-11 e13.

9. McKhann G, Drachman D, Folstein M, Katzman R, Price D, Stadlan EM. Clinical diagnosis of Alzheimer's disease: report of the NINCDS-ADRDA Work Group under the auspices of Department of Health and Human Services Task Force on Alzheimer's Disease. Neurology 1984;34:939-944.

10. Anand R, Gill KD, Mahdi AA. Therapeutics of Alzheimer's disease: Past, present and future. Neuropharmacology 2013.

11. Hampel H, Wilcock G, Andrieu S, et al. Biomarkers for Alzheimer's disease therapeutic trials. Progress in neurobiology 2011;95:579-593.

12. Braak H, Braak E. Frequency of stages of Alzheimer-related lesions in different age categories. Neurobiology of aging 1997;18:351-357.

13. Price JL, Morris JC. Tangles and plaques in nondemented aging and "preclinical" Alzheimer's disease. Annals of neurology 1999;45:358-368.

14. Querfurth HW, LaFerla FM. Alzheimer's disease. The New England journal of medicine 2010;362:329-344.

15. Aronson JK. Research priorities in biomarkers and surrogate end-points. British journal of clinical pharmacology 2012;73:900-907.

16. Shaw LM, Vanderstichele H, Knapik-Czajka M, et al. Cerebrospinal fluid biomarker signature in Alzheimer's disease neuroimaging initiative subjects. Annals of neurology 2009;65:403-413.

17. Fagan AM, Holtzman DM. Cerebrospinal fluid biomarkers of Alzheimer's disease. Biomarkers in medicine 2010;4:51-63.

18. Clark CM, Xie S, Chittams J, et al. Cerebrospinal fluid tau and beta-amyloid: how well do these biomarkers reflect autopsy-confirmed dementia diagnoses? Archives of neurology 2003;60:16961702.

19. Clark CM, Schneider JA, Bedell BJ, et al. Use of florbetapir-PET for imaging beta-amyloid pathology. JAMA : the journal of the American Medical Association 2011;305:275-283.

20. Rowe CC, Villemagne VL. Brain amyloid imaging. Journal of nuclear medicine technology 2013;41:11-18.

21. Jack CR, Jr. Alliance for aging research AD biomarkers work group: structural MRI. Neurobiology of aging 2011;32 Suppl 1:S48-57. 
22. Braak H, Braak E. Evolution of neuronal changes in the course of Alzheimer's disease. Journal of neural transmission Supplementum 1998;53:127-140.

23. Hoffman JM, Welsh-Bohmer KA, Hanson M, et al. FDG PET imaging in patients with pathologically verified dementia. Journal of nuclear medicine : official publication, Society of Nuclear Medicine 2000;41:1920-1928.

24. Silverman DH, Small GW, Chang CY, et al. Positron emission tomography in evaluation of dementia: Regional brain metabolism and long-term outcome. JAMA : the journal of the American Medical Association 2001;286:2120-2127.

25. Dubois B, Feldman HH, Jacova C, et al. Research criteria for the diagnosis of Alzheimer's disease: revising the NINCDS-ADRDA criteria. Lancet neurology 2007;6:734-746.

26. Dubois B, Feldman HH, Jacova C, et al. Revising the definition of Alzheimer's disease: a new lexicon. Lancet neurology 2010;9:1118-1127.

27. Sperling RA, Aisen PS, Beckett LA, et al. Toward defining the preclinical stages of Alzheimer's disease: recommendations from the National Institute on Aging-Alzheimer's Association workgroups on diagnostic guidelines for Alzheimer's disease. Alzheimer's \& dementia : the journal of the Alzheimer's Association 2011;7:280-292.

28. Albert MS, DeKosky ST, Dickson D, et al. The diagnosis of mild cognitive impairment due to Alzheimer's disease: recommendations from the National Institute on Aging-Alzheimer's Association workgroups on diagnostic guidelines for Alzheimer's disease. Alzheimer's \& dementia : the journal of the Alzheimer's Association 2011;7:270-279.

29. McKhann GM, Knopman DS, Chertkow H, et al. The diagnosis of dementia due to Alzheimer's disease: recommendations from the National Institute on Aging-Alzheimer's Association workgroups on diagnostic guidelines for Alzheimer's disease. Alzheimer's \& dementia : the journal of the Alzheimer's Association 2011;7:263-269.

30. Petersen RC. Mild cognitive impairment as a diagnostic entity. J Intern Med 2004;256:183-194.

31. Farias ST, Mungas D, Reed BR, Harvey D, DeCarli C. Progression of mild cognitive impairment to dementia in clinic- vs community-based cohorts. Archives of neurology 2009;66:1151-1157.

32. Petersen RC. Clinical practice. Mild cognitive impairment. The New England journal of medicine 2011;364:2227-2234.

33. Petersen RC, Smith GE, Waring SC, Ivnik RJ, Tangalos EG, Kokmen E. Mild cognitive impairment: clinical characterization and outcome. Archives of neurology 1999;56:303-308.

34. Busse A, Hensel A, Guhne U, Angermeyer MC, Riedel-Heller SG. Mild cognitive impairment: longterm course of four clinical subtypes. Neurology 2006;67:2176-2185.

35. Jungwirth S, Zehetmayer S, Hinterberger M, Tragl KH, Fischer P. The validity of amnestic MCI and non-amnestic MCI at age 75 in the prediction of Alzheimer's dementia and vascular dementia. International psychogeriatrics / IPA 2012;24:959-966.

36. Roberts RO, Geda YE, Knopman DS, et al. The incidence of MCI differs by subtype and is higher in men: the Mayo Clinic Study of Aging. Neurology 2012;78:342-351.

37. Schneider JA, Arvanitakis Z, Leurgans SE, Bennett DA. The neuropathology of probable Alzheimer disease and mild cognitive impairment. Annals of neurology 2009;66:200-208.

38. Manly JJ, Tang MX, Schupf N, Stern Y, Vonsattel JP, Mayeux R. Frequency and course of mild cognitive impairment in a multiethnic community. Annals of neurology 2008;63:494-506.

39. Jack CR, Jr., Albert MS, Knopman DS, et al. Introduction to the recommendations from the National Institute on Aging-Alzheimer's Association workgroups on diagnostic guidelines for Alzheimer's disease. Alzheimer's \& dementia : the journal of the Alzheimer's Association 2011;7:257-262. 
40. Hardy JA, Higgins GA. Alzheimer's disease: the amyloid cascade hypothesis. Science 1992;256:184185.

41. Hardy J, Selkoe DJ. The amyloid hypothesis of Alzheimer's disease: progress and problems on the road to therapeutics. Science 2002;297:353-356.

42. Jack CR, Jr., Knopman DS, Jagust WJ, et al. Hypothetical model of dynamic biomarkers of the Alzheimer's pathological cascade. Lancet neurology 2010;9:119-128.

43. Jack CR, Jr., Knopman DS, Jagust WJ, et al. Tracking pathophysiological processes in Alzheimer's disease: an updated hypothetical model of dynamic biomarkers. Lancet neurology 2013;12:207216.

44. Dumurgier J, Vercruysse 0, Paquet C, et al. Intersite variability of CSF Alzheimer's disease biomarkers in clinical setting. Alzheimer's \& dementia : the journal of the Alzheimer's Association 2013;9:406-413.

45. Lewczuk P, Beck G, Ganslandt 0, et al. International quality control survey of neurochemical dementia diagnostics. Neuroscience letters 2006;409:1-4.

46. Mattsson N, Andreasson U, Persson S, et al. The Alzheimer's Association external quality control program for cerebrospinal fluid biomarkers. Alzheimer's \& dementia : the journal of the Alzheimer's Association 2011;7:386-395 e386.

47. Verwey NA, van der Flier WM, Blennow K, et al. A worldwide multicentre comparison of assays for cerebrospinal fluid biomarkers in Alzheimer's disease. Annals of clinical biochemistry 2009;46:235-240.

48. Mattsson N, Andreasson U, Persson S, et al. CSF biomarker variability in the Alzheimer's Association quality control program. Alzheimer's \& dementia : the journal of the Alzheimer's Association 2013;9:251-261.

49. Hort J, Bartos A, Pirttila T, Scheltens P. Use of cerebrospinal fluid biomarkers in diagnosis of dementia across Europe. European journal of neurology : the official journal of the European Federation of Neurological Societies 2010;17:90-96. 


\section{PART I}

\section{BIOMARKERS FOR ALZHEIMER'S}

DISEASE IN SUBJECTS WITH MCI 



\title{
CHAPTER 2
}

\author{
BIOMARKERS AS PREDICTOR FOR CONVERSION FROM \\ MILD COGNITIVE IMPAIRMENT TO ALZHEIMER-TYPE DEMENTIA \\ IMPLICATIONS FOR TRIAL DESIGN
}

Journal of Alzheimer's Disease 2010;20:881-891

Ineke A. van Rossum, Stephanie Vos, Ron Handels, Pieter Jelle Visser 


\section{ABSTRACT}

Disease modifying drugs for Alzheimer's disease (AD) are likely to be most effective when given in non-demented subjects. In this review we summarized biomarkers in cerebrospinal fluid (CSF) and blood that can predict AD-type dementia in subjects with mild cognitive impairment (MCI). In addition we investigated whether these markers could reduce sample size and costs if used to select subjects for trials on the prevention of AD in subjects with MCI. A meta-analysis of markers that had been investigated in multiple studies showed that the combination of $A \beta 1-42$ and tau in CSF had the best predictive accuracy for AD (odds ratio (OR) 18.1, 95\% confidence interval (CI) 9.6-32.4). A $31-42$, total tau and phosphorylated tau in CSF also predicted conversion, but with lower accuracy (OR 7.5 to 8.1). Plasma level of $A \beta 1-40$ tended to predict conversion to AD (Cohen's delta $0.23,95 \% \mathrm{CI}$ -0.01-0.48, p 0.07). Plasma levels of $A \beta 1-42$, the ratio $A \beta 1-42 / A \beta 1-40$ and homocysteine did not predict outcome. In a fictive trial design, the use of the combination of $A \beta 1-42$ and tau in CSF in the selection of subjects could reduce sample size by $67 \%$ and trial costs by $60 \%$ compared to a trial in which unselected subjects with MCI would be enrolled. In conclusion, the combination of A $\beta 1-42$ and tau in CSF is useful to select subjects for trials that aim to slow down the progression from MCI to AD-type dementia. 


\section{INTRODUCTION}

Disease modifying drugs that may slow down the progression of Alzheimer's disease (AD) are currently under development. These drugs are likely to be most effective when given early in the course of the disease, but diagnostic criteria for AD in non-demented subjects are currently lacking. Predementia AD can not be accurately diagnosed by clinical criteria alone, such as criteria for mild cognitive impairment (MCI). ${ }^{1,2}$ Biomarkers may help to increase the diagnostic accuracy compared to clinical criteria, ${ }^{3}$ because pathological changes of $\mathrm{AD}$ can already be found in non-demented subjects. ${ }^{4}$ Which biomarkers would be most useful as inclusion criterion in trials with disease modifying drugs and to what extent these markers could reduce sample size and costs of such trials is however unknown.

The aim of the present study was to investigate which biomarkers could predict ADtype dementia in subjects with MCI and how the use of these biomarkers as an inclusion criterion for trials with drugs that aim to slow down the progression from MCI to AD-type dementia, could reduce sample size and costs. We limited our study to biomarkers in cerebrospinal fluid (CSF) and blood.

First, we will briefly summarize the pathophysiology in $A D$, since appropriate biomarkers should reflect AD pathology. ${ }^{5}$ Next, we will discuss biomarkers for the key pathological processes in $\mathrm{AD}$ in $\mathrm{CSF}$ and blood and discuss their use as predictor for progression to AD-type dementia in subjects with MCI. Of CSF and blood markers that have been investigated in at least 4 studies, we will calculate pooled measures of predictive accuracy. Finally, we will use the predictive accuracy of the best markers to calculate for a fictive trial how the use of these markers as an inclusion criterion will influence sample size and costs.

\section{PATHOPHYSIOLOGY OF AD}

Abnormalities in amyloid beta $(A \beta)$ processing play a central role in the pathophysiology of $\mathrm{AD}$. In $\mathrm{AD}, \mathrm{A} \beta$ peptides aggregate and form oligomers, fibrils and eventually plaques, $\mathrm{a}$ pathological hallmark of $A D$. Aggregated $A \beta$ is supposed to be neurotoxic and to initiate a cascade of events including oxidative stress, inflammation and dysregulation of lipid metabolism. This cascade eventually leads to loss of synapses and cell death, as shown in figure $1 .{ }^{6}$ Different isoforms of the $A \beta$ peptide are generated from the amyloid beta precursor protein $(A \beta P P)$. In $A D$, especially $A \beta 1-42$ seems to be important as it is more prone to aggregate compared to other isoforms. Cumulating evidence suggests that mainly the oligomer form of $A \beta$ is toxic. ${ }^{7}$ The increase in aggregated $A \beta$ may be caused by either increased production, disturbance of the post-translational modification or reduced clearance of $\mathrm{A} \beta$. Another pathological hallmark of AD are neurofibrillary tangles. These are aggregates of hyperphosphorylated tau and may contribute to neuronal dysfunction. Inflammatory processes, oxidative stress and alteration of lipid metabolism are though to occur as a consequence of $A \beta$ pathology, but may in turn further dysregulate $A \beta$ processing. 
The primary cause for dysregulation of the $A \beta$ metabolism is unknown and multiple factors may be involved, for example genetic or environmental factors.

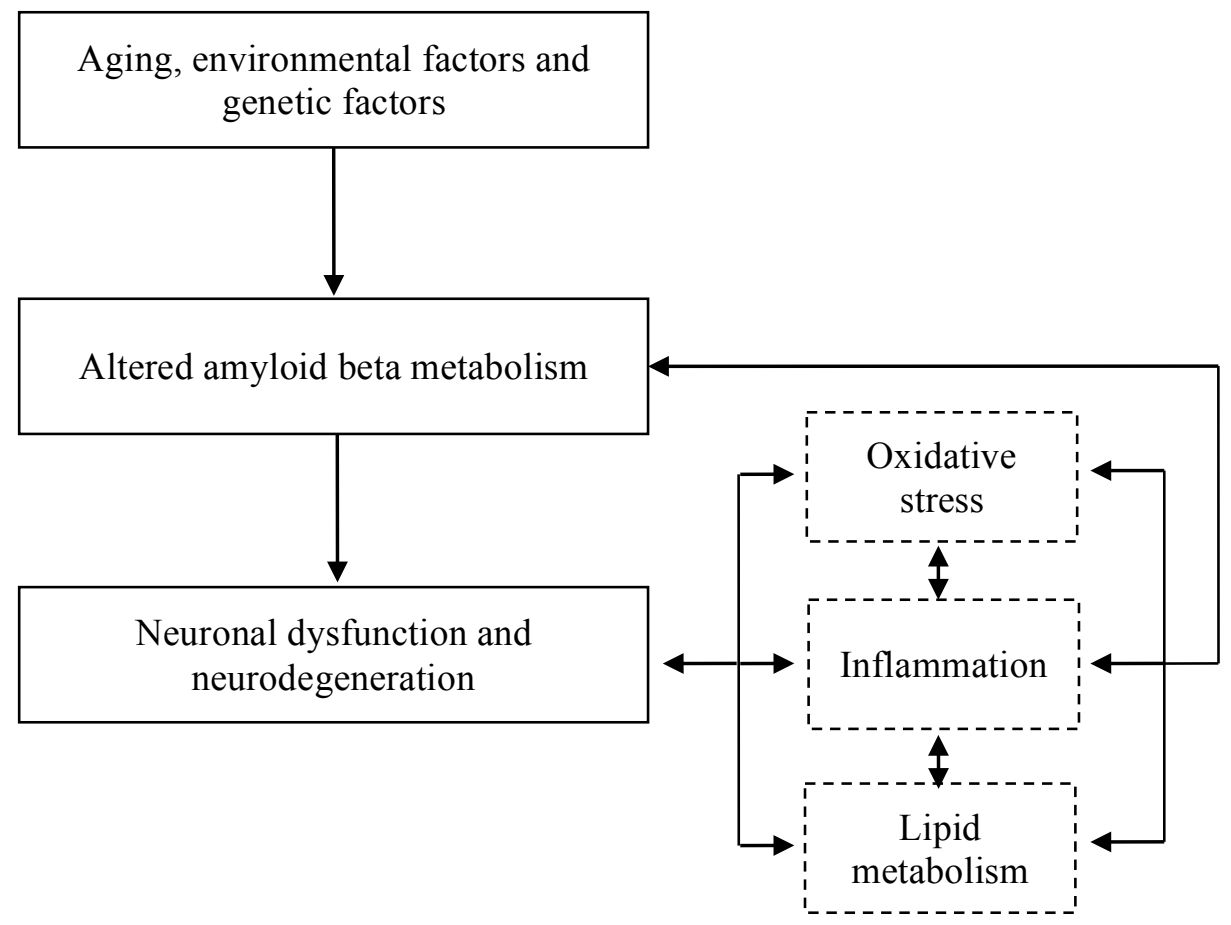

Figure 1. Pathophysiology of Alzheimer's disease

\section{Biomarkers for AD in subjects with MCI}

Many of the biochemical changes related to the pathophysiology of AD can be measured in vivo in body fluids.

\section{Markers for A $\beta$ processing in CSF}

Biomarkers for $A \beta$ processing in CSF include isoforms of the peptide itself as well as markers for its production and clearance. $A \beta$ peptides can be measured with assays that measure monomers or aggregated species, or with assays that are not specific for aggregation status. ${ }^{8-10}$ The concentration $A \beta 1-42$ correlates with the presence of amyloid plaques in the brain. ${ }^{11}$ Many studies have shown that in MCI subjects a low concentration of AB1-42 in CSF can predict conversion to AD-type dementia, as will be discussed in more detail below. Also a combination of different $A \beta$ isoforms in CSF may predict AD-type dementia in subjects with MCI. ${ }^{12}$ Production of $A \beta 1-42$ from $A \beta P P$ is dependent on the $\beta$ site A $\beta P P$ cleaving enzyme (BACE-1). One study showed that increased BACE1 activity could predict $\mathrm{AD}$-type dementia in $\mathrm{MCI}$ subjects. ${ }^{13}$ Of the peptides that may be involved in the clearance of $A \beta$ only few have been investigated in CSF of subjects with MCI. Levels of neprilysin were decreased in subjects with progressive MCI and mild AD-type dementia compared to controls. ${ }^{14} \mathrm{~A} \beta$ binding proteins may also influence $A \beta$ aggregation and a lower level of serum amyloid P component predicted AD-type dementia in MCI subjects. ${ }^{15}$ 


\section{Markers for $A \beta$ processing in plasma}

In plasma, abnormal $A \beta$ concentrations have been associated with $\mathrm{AD}$, although they do not correlate with the occurrence of $A \beta$ plaques in the brain. ${ }^{16,17}$ Four longitudinal studies have assessed predictive value of plasma $A \beta$ levels in MCI subjects as will be discussed in more detail below. In a small study, the A $\beta P P$ isoform ratio in platelets could predict conversion to AD-type dementia in MCI subjects with a sensitivity and specificity of $0.83 .{ }^{18}$

\section{Markers for neurodegeneration}

Many markers of neurodegeneration are available, but most of them are not specific for AD pathology. Concentrations of total tau (t-tau) and phosphorylated tau (p-tau) can be measured in CSF and correlate with the presence of neurofibrillary tangles. ${ }^{11,19}$ In MCI subjects increased CSF level of t-tau and p-tau were associated with an increased risk of progression to AD-type dementia as will be discussed below. In plasma, levels of tau are very low and no reliable assay is available yet. Other markers for neurodegeneration, like visinin-like protein, neurofilaments and GAP43 (neuromodulin) are increased in CSF of AD subjects compared to controls, ${ }^{20}$ but have not been tested as predictor for AD-type dementia in subjects with MCI.

\section{Markers for inflammation}

A wide range of inflammatory markers have been studied in blood and CSF of AD subjects, including cytokines, acute-phase proteins and complement factors, but none of these markers could discriminate between subjects with $\mathrm{AD}$ and healthy controls. ${ }^{21-24}$ In MCI, CSF tumor necrosis factor $\alpha$ (TNF- $\alpha$ ) was increased and transforming growth factor beta (TGF $\beta$ ) was decreased in subjects progressing to AD-type dementia compared to healthy controls, but not compared to subjects with stable MCI. ${ }^{25}$ Another study showed higher levels of TNF receptors in CSF as well as in plasma in MCI subjects that converted to AD-type dementia. ${ }^{26,27}$ Several other cytokines did not predict progression to AD-type dementia. ${ }^{25,28}$ Antibodies against $A \beta$ are found in CSF and plasma of AD subjects. Studies comparing levels of antibodies of $\mathrm{AD}$ subjects and healthy controls showed conflicting results. ${ }^{21}$ The possible use of antibodies as marker in MCI has not been studied yet.

\section{Markers for oxidative stress}

Some markers of lipid peroxidation, protein oxidation and nitrosylation and DNA and RNA oxidation have shown to be abnormal in CSF, plasma or peripheral blood cells of subjects with AD-type dementia. ${ }^{29}$ In MCI subjects, an increased level of F2-isoprostane, a marker of lipid peroxidation was associated with cognitive decline and progression to AD-type dementia. ${ }^{30}$

\section{Markers for lipid metabolism}

Serum cholesterol is suggested to be associated with an increased risk for AD, but results have been conflicting. ${ }^{31}$ In CSF, increased levels of $240 \mathrm{HC}$ and 27-hydroxycholestrol 
(270HC) were found in AD and MCI subjects compared to controls. ${ }^{32-35}$ In plasma, results have been conflicting. ${ }^{31}$ One study in MCI found that CSF levels of sulfatide and the ratio of sulfatide to phosphatidylinositol were decreased in very mildly demented subjects. ${ }^{36}$ However, the predictive accuracy for AD-type dementia in subjects with MCI has not yet been tested.

\section{Other markers}

Homocysteine

Increased plasma homocysteine may be associated with an increased risk for AD, although the exact pathophysiological mechanism behind this is not clear. ${ }^{37}$ Two studies found an association between homocysteine level and incidence of AD in the general population, ${ }^{38,39}$ another study found no association of plasma homocysteine level and the incidence of MCI. ${ }^{40}$ The predictive accuracy of homocysteine for AD-type dementia in subjects with MCI will be discussed in more detail below.

\section{Proteomics}

Unbiased approaches have been useful to select biomarkers in CSF or plasma that may differentiate between AD subjects and healthy controls. ${ }^{23}$ In CSF, a panel of 17 proteins and peptides in CSF could distinguish between subjects with stable MCI and subjects with MCI who progressed to AD-type dementia. ${ }^{41}$ In plasma, a combination of 18 different proteins in plasma predicted progression to AD-type dementia in MCI subjects with over $90 \%$ accuracy. ${ }^{42}$ In a subsequent study that tested for reproducibility of these markers diagnostic accuracy was however lower. ${ }^{43}$

\section{POOLED ANALYSIS OF PREDICTORS FOR AD IN SUBJECTS WITH MCI}

We performed a meta-analysis of markers that had been tested in at least 4 studies to obtain pooled estimates of the predictive accuracy for progression to AD-type dementia in subjects with MCI. These markers were A $\beta 1-42$, t-tau, and p-tau in CSF and A $\beta 1-40, A \beta 1-42$ and homocysteine in plasma. We identified studies through a systematic search in PubMed, Medline and Psychinfo (search terms available on request) and from the references of retrieved studies. If multiple studies reported on the same sample, we included the study with the longest follow up or the largest sample size. Data were pooled in a random effects model with STATA (version 9.2; Stata Corp, College Station, TX). For continuous variables we calculated Cohen's delta as outcome measure. ${ }^{44}$ For dichotomized scores we calculated the sensitivity, specificity, positive predictive value and odds ratio (OR). These dichotomized scores were based on cut-offs provided in the study. If different types of cut-off levels were used within one study, we chose predefined cut-offs over data-driven cut-offs. 


\section{$A \beta 1-42, t$-tau and $p$-tau in CSF}

We identified 19 studies $^{4,25,45-61}$ which provided unique data of CSF levels of A $\beta 1-42$, t-tau, $p$ tau as predictor for AD-type dementia in subjects with MCI (table 1). One multi-centre study may have included data that had been presented in other studies as well, ${ }^{56}$ but exclusion of this study did not change the outcome substantially and therefore the study was included in the analyses.

The mean conversion rate to AD-type dementia in the studies was $37 \%$ during a mean follow up of 2.5 years. Cohen's delta showed that A $\beta 1-42$, t-tau and p-tau were significant predictors of outcome (table 2). The highest odds ratio (OR) for AD-type dementia was found for the combination of A $31-42$ and t-tau with or without p-tau (OR 18.1, 95\% confidence interval (CI) 9.6-34.2). An abnormal combination was defined as an abnormal ratio of these markers or as an abnormal score for at least 2 markers. The combination $A \beta 1$ 42 with only p-tau had a slightly lower OR (17.5, 95\% CI 10-30.6). For the individual markers the OR ranged from $7.54(\mathrm{~A} \beta 1-42)$ to 8.05 (p-tau). For further analysis we selected the combination of A $\beta 1-42$ and t-tau, which was studied in 8 studies with a total of 1236 MCI subjects of which 454 converted to AD-type dementia. The sensitivity of this combination to predict AD-type dementia was 0.87 (95\% CI 0.80-0.95), the specificity 0.70 (95\% CI 0.57-0.83) and the positive predictive value 0.65 (95\% CI 0.53-0.77).

\section{$A \beta 1-40$ and $A \beta 1-42$ in plasma}

We selected 4 studies on the association of $A \beta$ plasma levels with conversion to AD-type dementia in subjects with $\mathrm{MCI}^{62-64}$ in which a total of 244 subjects with MCI was included (table 3). The mean conversion rate to AD-type dementia was $51 \%$ during a mean follow up of 4.0 years. The pooled effect sizes for $A \beta 1-40, A \beta 1-42$ and the ratio $A \beta$ 1-42/A $\beta 1-40$ and conversion to AD-type dementia are shown in table 2 . None of the effect sizes was statistically significant, although increased levels of A $31-40$ tended to be associated with conversion to AD-type dementia (Cohen's delta $0.23, \mathrm{p}=0.07$ (table 3 , figure 2)). No dichotomized scores were available.

\section{Homocysteine in plasma}

Four studies ${ }^{55,65-67}$ with in total 369 subjects investigated the predictive value of homocysteine for AD-type dementia in subjects with MCI (table 3). Mean conversion rate to AD-type dementia was $37 \%$ during a mean follow up of 3.3 years. Homocysteine did not predict AD-type dementia (Cohen's delta 0.21, table 2). No dichotomized scores were available. 


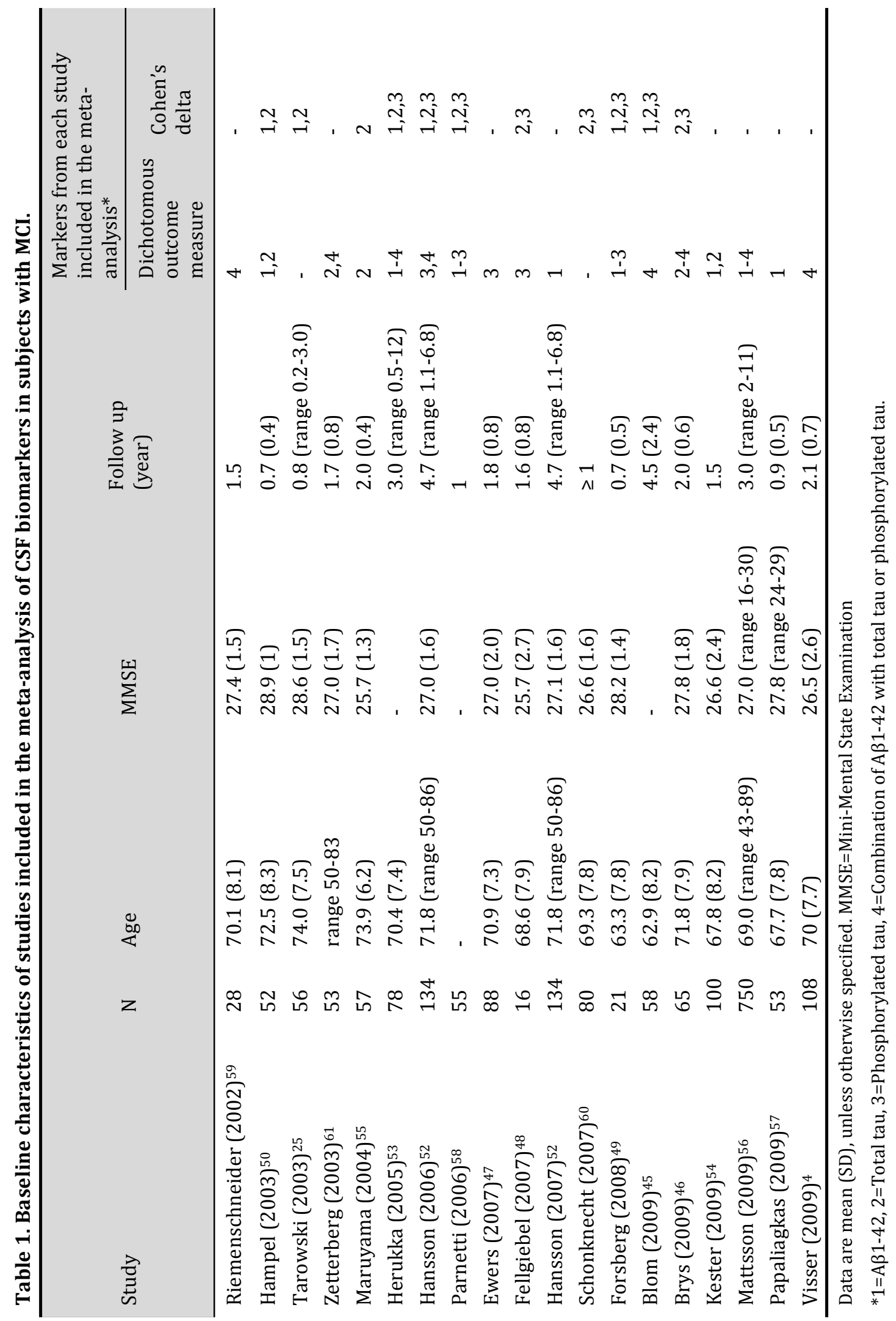


Table 2. Effect sizes of biomarkers in CSF or plasma for prediction of Alzheimer's disease in subjects with MCI

\begin{tabular}{lllll}
\hline & $\begin{array}{l}\text { Number of } \\
\text { studies }\end{array}$ & $\begin{array}{l}\text { Number of subjects } \\
\text { (AD at follow-up/ } \\
\text { no-AD at follow-up) }\end{array}$ & $\begin{array}{l}\text { Cohen's delta } \\
(95 \% \mathrm{CI})\end{array}$ & P-value \\
\hline CSF & 7 & $172 / 252$ & $0.84(0.45-1.24)$ & $<0.001$ \\
A 1 -42 & 11 & $238 / 404$ & $0.90(0.62-1.17)$ & $<0.001$ \\
T-tau & 8 & $161 / 316$ & $1.21(0.85-1.57)$ & $<0.001$ \\
P-tau & & & &
\end{tabular}

Plasma

\begin{tabular}{lllll}
$\mathrm{A} \beta 1-40$ & 4 & $133 / 190$ & $0.23(-0.01-0.48)$ & 0.07 \\
$\mathrm{~A} \beta 1-42$ & 4 & $133 / 190$ & $0.03(-0.31-0.38)$ & 0.55 \\
Ratio $\mathrm{A} \beta 1-42 / \mathrm{A} \beta 1-40$ & 4 & $133 / 190$ & $-0.13(-0.37-0.12)$ & 0.25 \\
Homocysteine & 4 & $138 / 231$ & $0.21(-0.08-0.50)$ & 0.15 \\
\hline
\end{tabular}

$\mathrm{AD}=$ Alzheimer type dementia; $\mathrm{CSF}=$ Cerebrospinal fluid; $\mathrm{A} \beta 1-42=$ Amyloid beta peptide 1-42; $\mathrm{A} \beta 1-40=$ Amyloid beta peptide 1-40; T-tau=Total tau; $\mathrm{P}=$ tau=Phosphorylated tau.

Table 3. Characteristics of studies included in the meta-analysis of plasma markers in subjects with MCI

\begin{tabular}{|c|c|c|c|c|}
\hline Study & $\mathrm{N}$ & Age & MMSE & Follow up (year) \\
\hline \multicolumn{5}{|l|}{$A \beta 1-42$ and $A \beta 1-40$} \\
\hline Hansson (2008) ${ }^{63}$ & 117 & 69.7 , range $50-85$ & $27.0(1.6)$ & 5.2 \\
\hline Hansson $(2008)^{63}$ & 110 & 62.9, range $47-79$ & $27.0(1.6)$ & Range $2-4$ \\
\hline Lopez $(2008)^{64}$ & 42 & $79.8(4.3)$ & - & 4.5 \\
\hline Cammarata (2009) 62 & 79 & $75.2(5.2)$ & $>26$ & 2.0 \\
\hline \multicolumn{5}{|l|}{ Homocysteine } \\
\hline Maruyama (2004)55 & 57 & $73.9(5.7)$ & $25.6(1.3)$ & 2.0 \\
\hline Annerbo $(2006)^{65}$ & 93 & $65.0(8.5)$ & $26.4(2.6)$ & 6.0 \\
\hline Gabryelewicz (2007) ${ }^{66}$ & 105 & $69.3(6.9)$ & $27.3(1.8)$ & 3.0 \\
\hline Siuda $(2009)^{67}$ & 55 & $70.8(5.7)$ & - & 1.0 \\
\hline
\end{tabular}

Data are mean (SD), unless otherwise specified. MMSE=Mini Mental State Examination; A 1 -42= Amyloid beta peptide 1-42; A 1-40=Amyloid beta peptide 1-40. 


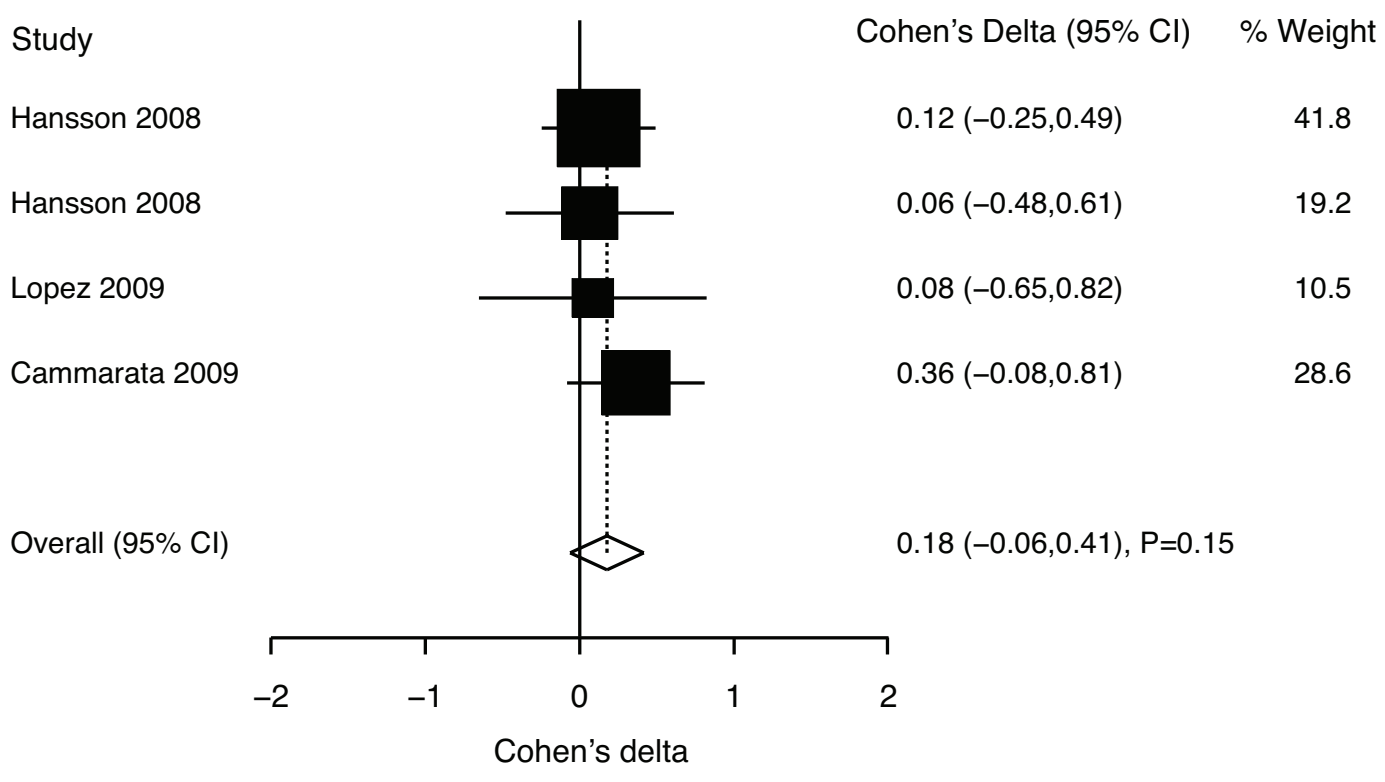

Figure 2. Pooled Cohen's delta of Aß-40 plasma levels for the prediction of AD-type dementia in subjects with MCI.

A positive Cohen's delta indicates that increased plasma levels are associated with an increased risk for AD.

\section{USE OF CSF BIOMARKERS IN SELECTION SUBJECTS FOR DRUG TRIALS}

In the previous section we showed that the combination of $A \beta 1-42$ and tau in CSF had the best predictive accuracy to predict conversion to AD-type dementia in subjects with MCI. In this section we will investigate how the use of these markers for the selection of subjects can reduce sample size and costs of a trial with a drug that could slow down the progression from MCI to AD-type dementia. We compare the costs of 2 strategies. The first strategy is a trial in which subjects with MCI are included. The second strategy is a trial in which subjects are enrolled with $\mathrm{MCI}$ and an abnormal combination of Aß1-42 and tau in CSF. Calculations will be performed from the perspective of the performer of the trial and input estimates are mainly based on expert opinion.

The hypothetical trial is a 3-year placebo-controlled trial with $50 \%$ on active treatment to examine the effectiveness of an oral drug on the delay of progression to ADtype dementia in subjects with MCI. Subjects would be selected from a memory clinic setting, with MCI defined as an abnormal performance on neuropsychological tests in the absence of dementia. ${ }^{68}$ Costs needed to apply the trial inclusion and exclusion criteria (including cognitive tests and imaging) are estimated on $€ 700$ per patient. Costs for the collection and analysis of CSF are estimated on $€ 324$. The costs of the treatment phase of the trial were estimated on $€ 5800$ per included patient. This included costs for 7 assessments with clinical and cognitive testing and blood analysis and costs related to drugs, drug supply, approval of the medical ethical committee and subject reimbursement. Costs related to side effects and adverse events were not included in the analysis. 
The number of subjects was chosen such that the study could detect a relative decrease of $20 \%$ in conversion rate to AD-type dementia over the 3-year period, with a power of $90 \%$, a 2 -sided alpha of $5 \%$, and a drop-out rate of $30 \%$. The number of subjects to be included would depend on the conversion rate to AD-type dementia in each strategy. Based on the meta-analysis above, we estimated that the 3-year conversion rate would be $40 \%$ in subjects with MCI and 70\% in subjects with MCI and an abnormal A $\beta$ and tau in CSF. Based on these conversion rates, the number of subjects to be included in the trial would be 2160 for a study with only subjects with MCI (strategy 1) and 714 for a study with subjects with MCI and an abnormal combination of Aß1-42 and tau in CSF (strategy 2). Based on a sensitivity of 0.87 and a specificity of 0.70 of the CSF test (see previous section) and a failure rate of lumbar puncture of 10\%, 1503 subjects with MCI should undergo lumbar puncture in order to include 714 subjects with an abnormal combination of AB1-42 and tau in CSF. The strategies are visualized in figure 3. This figure shows the rounded number of subjects for each arm, while costs were calculated based on the unrounded number of subjects.

The trial costs of strategy 1 with only subjects with MCI included would be $€$ $14,040,000$ (pre-trial screening costs and trial costs for 2160 subjects, figure 3 ). The trial costs of strategy 2 in which subjects with MCI and an abnormal combination of A $\beta 1-42$ and tau in CSF are included would be $€ 5,681,580$ (pre-trial screening costs and CSF collection and analysis costs for 1503 subjects, and trial costs for 714 subjects, figure 3 ). This is a reduction in costs of $60 \%$ compared to strategy 1.

A one-way sensitivity analysis using the lower bound of the confidence interval of the test-sensitivity ( 0.80 instead of 0.87 ) mentioned above would increase the number of subjects with 208 and trial costs with $€ 536,194$ (reduction costs compared to strategy 1 of $56 \%$ instead of $60 \%$ ). Using the lower bound of the specificity ( 0.57 instead of 0.70 ) would increase the number of subjects with 261 and trial costs with $€$ 1,704,852 (reduction costs compared to strategy 1 of $47 \%$ ). If the lower bound of positive predictive value was used (0.58 instead of 0.70), 839 extra subjects needed to be included and trial costs would increase with $3,171,554$ (reduction costs compared to strategy 1 of $37 \%$ ). A CSF test-price increase of $25 \%$ resulted in an increase of $€ 121,743$ of the total trial costs (reduction costs compared to strategy 1 of 59\%). If trial costs would double to $€ 11,600$ per patient strategy 2 would reduce costs by $63 \%$ compared to strategy 1 . 


\begin{tabular}{|c|c|c|c|c|c|c|c|}
\hline & & & & Costs & & & \\
\hline & & & $\begin{array}{l}\text { Number of } \\
\text { subjects }\end{array}$ & Pre-trial & Test & Trial & Total \\
\hline Strategy 1 & & & & & & & \\
\hline 2160 subjects with & AD positive $(40 \%)$ & & $864^{\#}$ & $€ 700$ & $€ 0$ & $€ 5,800$ & \\
\hline & AD negative $(60 \%)$ & & $1296^{\#}$ & $€ 700$ & $€ 0$ & $€ 5,800$ & \\
\hline Strategy 2 & AD positive $(40 \%)$ & Test positive & $471^{\#}$ & $€ 700$ & $€ 324$ & $€ 5,800$ & \\
\hline 1503 subjects tested & & Test negative & 70 & $€ 700$ & $€ 324$ & & \\
\hline & AD negative $(60 \%)$ & $\begin{array}{l}\text { Test positive } \\
\text { Test negative }\end{array}$ & $\frac{243^{\#}}{568}$ & $\begin{array}{l}€ 700 \\
€ 700\end{array}$ & $\begin{array}{l}€ 324 \\
€ 324\end{array}$ & $€ 5,800$ & \\
\hline & & & & & & & $€ 5,681,580$ * \\
\hline Strategy 3 & AD positive $(40 \%)$ & Test positive & $699^{\#}$ & $€ 700$ & $€ 100$ & $€ 5,800$ & \\
\hline $\begin{array}{l}3013 \text { subjects tested } \\
\text { for plasma marker }\end{array}$ & & Test negative & 506 & $€ 700$ & $€ 100$ & & \\
\hline & AD negative $(60 \%)$ & Test positive & $633^{\#}$ & $€ 700$ & $€ 100$ & $€ 5,800$ & \\
\hline & & Test negative & 1175 & $€ 700$ & $€ 100$ & & $€ 10,134,527$ \\
\hline
\end{tabular}

Figure 3. Costs related to different recruitment strategies in a hypothetical trial.

Strategy 1 refers to a trial in which subjects with MCI are included and strategy 2 to a trial in which subjects with MCI and an abnormal amyloid beta peptide 1-42/tau ratio in CSF are included. It is assumed that $40 \%$ of the subjects with MCI would convert to $\mathrm{AD}$ at follow-up. The number of subjects with a positive and negative test result are based on a sensitivity of 0.87 and a specificity of 0.70 as explained in the text. The rounded number of subjects in each arm is shown, while costs were calculated based on the unrounded numbers of subjects. Test positive=number of subjects with abnormal CSF ratio; test negative=number of subjects with normal CSF ratio; test failed=number of subjects in which lumber puncture failed (10\% of sample). \#Subjects included in trial

\section{DISCUSSION}

Our main findings are that the combination of A $\beta 1-42$ with total tau in CSF is the best predictor for conversion from MCI to AD-type dementia and that use of these markers in the selection of subjects for trials with drugs that aim to prevent the progression from MCI to AD trials can substantially reduce the sample size and trial costs.

Although a large variety of biomarkers for $A D$ can quantify dysregulation of $A \beta$ metabolism or secondary processes in AD like neurodegeneration, inflammation, oxidative stress and altered lipid metabolism in CSF and blood, only few of them have been tested as predictor for AD-type dementia in subjects with $\mathrm{MCI}$ in more than 4 independent studies. Among the markers tested in multiple studies, the combination A 1 1-42 with t-tau in CSF had the best predictive accuracy. CSF levels of A $\beta 1-42$, t-tau and p-tau could also predict conversion to AD-type dementia but the accuracy was lower. In plasma, increased levels of $A \beta 1-40$ tended to be associated with an increased risk for AD-type dementia, although the effect size of 0.23 was small. $A \beta 1-42$, the ratio of $A \beta 1-42 / A \beta 1-40$, and homocysteine levels did not predict AD-type dementia.

In our hypothetical trial on the prevention of progression from MCI to AD-type dementia, use of A 1-42 and tau in CSF for the selection of subjects could reduce trial costs 
up to $60 \%$ relative to a trial that would include subjects with MCI that were not selected by CSF markers. This reduction largely resulted from the fact that fewer subjects were needed to be enrolled in order to obtain the required statistical power. The sample size could be smaller because the conversion rate in subjects with MCI and abnormal A $\beta 1-42$ and tau levels $(70 \%)$ was much higher compared to that in unselected subjects with MCI (40\%). The sensitivity analysis confirmed the strong effect of conversion rate on costs, as the cost reduction of a model with a conversion rate of $58 \%$ in subjects with abnormal A $\beta 1-42$ and tau levels would be much lower (37\%). The sensitivity analyses also showed that the costs of the CSF test had only a minor influence on the cost reduction and that benefits would be larger if the costs for conducting the trial increased.

Our study has several limitations. We may have overestimated the predictive accuracy of the CSF markers as some studies included in the meta-analysis dichotomized scores according to the best cut off in their study. There were differences in study design between the pooled studies but it was outside the scope of the present paper to explore the effect of this variability on the predictive accuracy. Our meta-analysis was based on relatively few studies, which reduced the accuracy of the parameter estimates. Therefore, we used lower bound estimates in the sensitivity analyses of the cost calculations. Still, these were one-way sensitivity analyses and not full sensitivity analyses. Our cost-calculation did not take into account costs associated with side effects of lumbar puncture. The most common side effect is post-lumbar headache in less than $3 \%$ of the subjects, which occasionally needs intervention. ${ }^{69}$

We calculated the effect on costs when CSF markers were used to select subjects for treatment trials. However, when CSF markers are used to select subjects for treatment outside the clinical trial setting, the cost-benefit could be markedly different. In that case, costs need to be calculated from a societal perspective, which includes all costs and effects related to the disease, such as medication use, caregiver time, patient and caregiver quality of life and institutionalization. The cost reduction of the use of CSF tests in the selection of subjects for treatment in such a setting is likely to be much lower as subjects with a falsenegative diagnosis or subjects that could not undergo the diagnostic test would not receive treatment and could not benefit from the reduction in societal costs associated with treatment.

\section{Clinical implications}

Our findings suggest that the design of trials on the prevention of progression from MCI to AD-type dementia could have a higher cost-benefit ratio if subjects are included based on abnormal CSF markers. A possible disadvantage of the use of CSF markers, however, is that the logistics of such trial could become more complex. CSF collection may not be available in all settings and subjects may refuse to undergo lumbar puncture or have a contraindication for it. In addition, facilities should be set-up for central analysis of CSF samples, which can provide a standardized cut-off. ${ }^{70}$ If lumbar puncture is performed on a large scale, rare side effects may become apparent. 


\section{Future perspectives}

Since lumbar puncture is relatively invasive, a plasma biomarker for AD would be more attractive. So far attempts to develop an accurate plasma AD marker have been unsuccessful. It seems unlikely that new biomarkers in either CSF or blood may reach or improve the diagnostic accuracy of that of A $1-42$ and tau in CSF in the near future. New markers that have been tested in subjects with AD-type dementia versus healthy control have generally yielded a lower diagnostic accuracy compared to A $\beta 1-42$ and tau in CSF. A promising alternative may be the combination of different plasma or CSF markers or gene expression profiles, but so far replication of such multiparameter approaches has been difficult. ${ }^{43}$ Development of diagnostic markers from proof of concept to large-scale application may take up to 15 years, as was the case for A $\beta 1-42$ and tau in CSF. Future studies should compare the use of CSF makers in trials with that of other diagnostic modalities such as PET or MRI imaging. Finally, it should be investigated whether biomarkers could increase the cost-benefit ratio in the selection of subjects in prevention trials in subjects without MCI or outside a clinical setting, in which the conversion rate to AD-type dementia is much lower. 


\section{REFERENCES}

1. Petersen RC, Doody R, Kurz A, et al. Current Concepts in Mild Cognitive Impairment. Arch Neurol 2001;58(12):1985-1992.

2. Visser PJ, Verhey FR. Mild cognitive impairment as predictor for Alzheimer's disease in clinical practice: effect of age and diagnostic criteria. Psychol Med 2008 Jan;38(1):113-122.

3. Dubois B, Feldman HH, Jacova C, et al. Research criteria for the diagnosis of Alzheimer's disease: revising the NINCDS-ADRDA criteria. Lancet Neurol 2007 Aug;6(8):734-746.

4. Visser PJ, Verhey F, Knol DL, et al. Prevalence and prognostic value of CSF markers of Alzheimer's disease pathology in patients with subjective cognitive impairment or mild cognitive impairment in the DESCRIPA study: a prospective cohort study. The Lancet Neurology 2009;8(7):619-627.

5. Consensus report of the Working Group on: "Molecular and Biochemical Markers of Alzheimer's Disease". The Ronald and Nancy Reagan Research Institute of the Alzheimer's Association and the National Institute on Aging Working Group. Neurobiol Aging 1998 Mar;19(2):109-116.

6. Hardy J, Selkoe DJ. The amyloid hypothesis of Alzheimer's disease: progress and problems on the road to therapeutics. Science 2002 Jul 19;297(5580):353-356.

7. Walsh DM, Selkoe DJ. A beta oligomers - a decade of discovery. J Neurochem 2007 Jun;101(5):11721184.

8. Blennow K, Hampel H. CSF markers for incipient Alzheimer's disease. Lancet Neurol 2003 Oct;2(10):605-613.

9. Funke SA, Birkmann E, Henke F, et al. Single particle detection of Abeta aggregates associated with Alzheimer's disease. Biochem Biophys Res Commun 2007 Dec 28;364(4):902-907.

10. Portelius E, Westman-Brinkmalm A, Zetterberg H, Blennow K. Determination of beta-amyloid peptide signatures in cerebrospinal fluid using immunoprecipitation-mass spectrometry. $J$ Proteome Res 2006 Apr;5(4):1010-1016.

11. Tapiola T, Alafuzoff I, Herukka SK, et al. Cerebrospinal fluid beta-amyloid 42 and tau proteins as biomarkers of Alzheimer-type pathologic changes in the brain. Arch Neurol 2009 Mar;66(3):382389.

12. Hoglund K, Hansson O, Buchhave P, et al. Prediction of Alzheimer's disease using a cerebrospinal fluid pattern of C-terminally truncated beta-amyloid peptides. Neurodegener Dis 2008;5(5):268276.

13. Zetterberg H, Andreasson U, Hansson O, et al. Elevated cerebrospinal fluid BACE1 activity in incipient Alzheimer disease. Arch Neurol 2008 Aug;65(8):1102-1107.

14. Maruyama M, Higuchi M, Takaki Y, et al. Cerebrospinal fluid neprilysin is reduced in prodromal Alzheimer's disease. Ann Neurol 2005 Jun;57(6):832-842.

15. Verwey NA, Schuitemaker A, van der Flier WM, et al. Serum amyloid p component as a biomarker in mild cognitive impairment and Alzheimer's disease. Dement Geriatr Cogn Disord 2008;26(6):522527.

16. Fagan AM, Mintun MA, Mach RH, et al. Inverse relation between in vivo amyloid imaging load and cerebrospinal fluid Abeta42 in humans. Ann Neurol 2006 Mar;59(3):512-519.

17. Freeman SH, Raju S, Hyman BT, Frosch MP, Irizarry MC. Plasma Abeta levels do not reflect brain Abeta levels. J Neuropathol Exp Neurol 2007 Apr;66(4):264-271.

18. Borroni B, Colciaghi F, Caltagirone C, et al. Platelet amyloid precursor protein abnormalities in mild cognitive impairment predict conversion to dementia of Alzheimer type: a 2-year follow-up study. Arch Neurol 2003 Dec;60(12):1740-1744.

19. Buerger K, Ewers M, Pirttila T, et al. CSF phosphorylated tau protein correlates with neocortical neurofibrillary pathology in Alzheimer's disease. Brain 2006 Nov;129(Pt 11):3035-3041. 
20. Blennow K. Cerebrospinal fluid protein biomarkers for Alzheimer's disease. NeuroRx 2004 Apr;1(2):213-225.

21. Britschgi M, Wyss-Coray T. Systemic and acquired immune responses in Alzheimer's disease. Int Rev Neurobiol 2007;82:205-233.

22. Craig-Schapiro R, Fagan AM, Holtzman DM. Biomarkers of Alzheimer's disease. Neurobiol Dis 2009 Aug;35(2):128-140.

23. Song F, Poljak A, Smythe GA, Sachdev P. Plasma biomarkers for mild cognitive impairment and Alzheimer's disease. Brain Res Rev 2009 Oct;61(2):69-80.

24. Teunissen CE, de VJ, Steinbusch HW, De BC. Biochemical markers related to Alzheimer's dementia in serum and cerebrospinal fluid. Neurobiol Aging 2002 Jul;23(4):485-508.

25. Tarkowski E, Andreasen N, Tarkowski A, Blennow K. Intrathecal inflammation precedes development of Alzheimer's disease. J Neurol Neurosurg Psychiatry 2003;74(9):1200-1205.

26. Buchhave P, Janciauskiene S, Zetterberg H, Blennow K, Minthon L, Hansson O. Elevated plasma levels of soluble CD40 in incipient Alzheimer's disease. Neurosci Lett 2009 Jan 23;450(1):56-59.

27. Buchhave P, Zetterberg H, Blennow K, Minthon L, Janciauskiene S, Hansson O. Soluble TNF receptors are associated with Abeta metabolism and conversion to dementia in subjects with mild cognitive impairment. Neurobiol Aging 2008 Dec 12; doi:10.1016/j.neurobiolaging.2008.10.012.

28. Galimberti D, Schoonenboom N, Scheltens P, et al. Intrathecal chemokine synthesis in mild cognitive impairment and Alzheimer disease. Arch Neurol 2006 Apr;63(4):538-543.

29. Mangialasche F, Polidori MC, Monastero R, et al. Biomarkers of oxidative and nitrosative damage in Alzheimer's disease and mild cognitive impairment. Ageing Res Rev 2009 Oct;8(4):285-305.

30. de Leon MJ, Mosconi L, Li J, et al. Longitudinal CSF isoprostane and MRI atrophy in the progression to AD. J Neurol 2007 Dec;254(12):1666-1675.

31. Solfrizzi V, D'Introno A, Colacicco AM, et al. Circulating biomarkers of cognitive decline and dementia. Clin Chim Acta 2006 Feb;364(1-2):91-112.

32. Bretillon L, Siden A, Wahlund LO, et al. Plasma levels of 24S-hydroxycholesterol in patients with neurological diseases. Neurosci Lett 2000 Oct 27;293(2):87-90.

33. Leoni V, Shafaati M, Salomon A, Kivipelto M, Bjorkhem I, Wahlund LO. Are the CSF levels of 24Shydroxycholesterol a sensitive biomarker for mild cognitive impairment? Neurosci Lett 2006 Apr 10;397(1-2):83-87.

34. Papassotiropoulos A, Lutjohann D, Bagli M, et al. 24S-hydroxycholesterol in cerebrospinal fluid is elevated in early stages of dementia. J Psychiatr Res 2002 Jan;36(1):27-32.

35. Schonknecht P, Lutjohann D, Pantel J, et al. Cerebrospinal fluid 24S-hydroxycholesterol is increased in patients with Alzheimer's disease compared to healthy controls. Neurosci Lett 2002 May 10;324(1):83-85.

36. Han X, Fagan AM, Cheng H, Morris JC, Xiong C, Holtzman DM. Cerebrospinal fluid sulfatide is decreased in subjects with incipient dementia. Ann Neurol 2003 Jul;54(1):115-119.

37. Seshadri S. Elevated plasma homocysteine levels: risk factor or risk marker for the development of dementia and Alzheimer's disease? J Alzheimers Dis 2006 Aug;9(4):393-398.

38. Ravaglia G, Forti P, Maioli F, et al. Homocysteine and folate as risk factors for dementia and Alzheimer disease. Am J Clin Nutr 2005 Sep;82(3):636-643.

39. Seshadri S, Beiser A, Selhub J, et al. Plasma homocysteine as a risk factor for dementia and Alzheimer's disease. N Engl J Med 2002 Feb 14;346(7):476-483.

40. Reitz C, Tang MX, Miller J, Green R, Luchsinger JA. Plasma homocysteine and risk of mild cognitive impairment. Dement Geriatr Cogn Disord 2009;27(1):11-17. 
41. Simonsen AH, McGuire J, Hansson 0, et al. Novel panel of cerebrospinal fluid biomarkers for the prediction of progression to Alzheimer dementia in patients with mild cognitive impairment. Arch Neurol 2007 Mar;64(3):366-370.

42. Ray S, Britschgi M, Herbert C, et al. Classification and prediction of clinical Alzheimer's diagnosis based on plasma signaling proteins. Nat Med 2007 Nov;13(11):1359-1362.

43. Soares HD, Chen Y, Sabbagh M, Rohrer A, Schrijvers E, Breteler M. Identifying early markers of Alzheimer's disease using quantitative multiplex proteomic immunoassay panels. Ann N Y Acad Sci 2009 Oct;1180:56-67.

44. Cohen J. A power primer. Psychological Bulletin 1992;112(1):155-159.

45. Blom ES, Giedraitis V, Zetterberg H, et al. Rapid progression from mild cognitive impairment to Alzheimer's disease in subjects with elevated levels of tau in cerebrospinal fluid and the APOE epsilon4/epsilon4 genotype. Dement Geriatr Cogn Disord 2009;27(5):458-464.

46. Brys M, Pirraglia E, Rich K, et al. Prediction and longitudinal study of CSF biomarkers in mild cognitive impairment. Neurobiol Aging 2009 May;30(5):682-690.

47. Ewers MP, Buerger KM, Teipel SJM, et al. Multicenter assessment of CSF-phosphorylated tau for the prediction of conversion of MCI. Neurology 2007;69(24):2205-2212.

48. Fellgiebel A, Scheurich A, Bartenstein P, Müller MJ. FDG-PET and CSF phospho-tau for prediction of cognitive decline in mild cognitive impairment. Psychiatry Research: Neuroimaging 2007;155(2):167-171.

49. Forsberg A, Engler H, Almkvist O, et al. PET imaging of amyloid deposition in patients with mild cognitive impairment. Neurobiology of Aging 2008;29(10):1456-1465.

50. Hampel H, Teipel SJ, Fuchsberger T, et al. Value of CSF [beta]-amyloid1-42 and tau as predictors of Alzheimer's disease in patients with mild cognitive impairment. Molecular Psychiatry 2003;9(7):705-710.

51. Hansson O, Zetterberg H, Buchhave P, et al. Prediction of Alzheimer's Disease Using the CSF Ab42/Ab40 Ratio in Patients with Mild Cognitive Impairment. Dementia and Geriatric Cognitive Disorders 2007;23(5):316-320.

52. Hansson O, Zetterberg H, Buchhave P, Londos E, Blennow K, Minthon L. Association between CSF biomarkers and incipient Alzheimer's disease in patients with mild cognitive impairment: a followup study. The Lancet Neurology 2006;5(3):228-234.

53. Herukka SK, Hallikainen MMDP, Soininen HMDP, Pirttila TMDP. CSF A[beta]42 and tau or phosphorylated tau and prediction of progressive mild cognitive impairment. Neurology 2005;64(7):1294-1297.

54. Kester MI, Verwey NA, van Elk EJ, Blankenstein MA, Scheltens P, van der Flier WM. Progression from MCI to AD: Predictive value of CSF A[beta]42 is modified by APOE genotype. Neurobiology of Aging 9 A.D.;doi:10.1016/j.neurobiolaging.2009.08.006.

55. Maruyama M, Matsui T, Tanji H, et al. Cerebrospinal fluid tau protein and periventricular white matter lesions in patients with mild cognitive impairment: implications for 2 major pathways. Arch Neurol 2004 May;61(5):716-720.

56. Mattsson N, Zetterberg H, Hansson O, et al. CSF Biomarkers and Incipient Alzheimer Disease in Patients With Mild Cognitive Impairment. JAMA 2009;302(4):385-393.

57. Papaliagkas VT, Anogianakis G, Tsolaki MN, Koliakos G, Kimiskidis VK. Progression of Mild Cognitive Impairment to Alzheimer's Disease: Improved Diagnostic Value of the Combined Use of N200 Latency and b-Amyloid(1--42) Levels. Dementia and Geriatric Cognitive Disorders 2009;28(1):30-35. 
58. Parnetti L, Lanari A, Silvestrelli G, Saggese E, Reboldi P. Diagnosing prodromal Alzheimer's disease: Role of CSF biochemical markers. Mechanisms of Ageing and Development 2006;127(2):129-132.

59. Riemenschneider M, Lautenschlager N, Wagenpfeil S, Diehl J, Drzezga A, Kurz A. Cerebrospinal Fluid Tau and \{beta\}-Amyloid 42 Proteins Identify Alzheimer Disease in Subjects With Mild Cognitive Impairment. Arch Neurol 2002;59(11):1729-1734.

60. Schonknecht P, Pantel J, Kaiser E, Thomann P, Schröder J. Increased tau protein differentiates mild cognitive impairment from geriatric depression and predicts conversion to dementia. Neuroscience Letters 2007;416(1):39-42.

61. Zetterberg H, Wahlund LO, Blennow K. Cerebrospinal fluid markers for prediction of Alzheimer's disease. Neuroscience Letters 2003;352(1):67-69.

62. Cammarata S, Borghi R, Giliberto L, et al. Amyloid-beta42 plasma levels are elevated in amnestic mild cognitive impairment. J Alzheimers Dis 2009;18(2):267-271.

63. Hansson O, Zetterberg H, Vanmechelen E, et al. Evaluation of plasma Abeta(40) and Abeta(42) as predictors of conversion to Alzheimer's disease in patients with mild cognitive impairment. Neurobiol Aging 2008 May 16;31(3):357-367.

64. Lopez OL, Kuller LH, Mehta PD, et al. Plasma amyloid levels and the risk of AD in normal subjects in the Cardiovascular Health Study. Neurology 2008 May 6;70(19):1664-1671.

65. Annerbo S, Wahlund LO, Lokk J. The significance of thyroid-stimulating hormone and homocysteine in the development of Alzheimer's disease in mild cognitive impairment: a 6-year follow-up study. Am J Alzheimers Dis Other Demen 2006 Jun;21(3):182-188.

66. Gabryelewicz T, Styczynska M, Luczywek E, et al. The rate of conversion of mild cognitive impairment to dementia: predictive role of depression. Int J Geriatr Psychiatry 2007 Jun;22(6):563567.

67. Siuda J, Gorzkowska A, Patalong-Ogiewa M, et al. From mild cognitive impairment to Alzheimer's disease - influence of homocysteine, vitamin B12 and folate on cognition over time: results from one-year follow-up. Neurol Neurochir Pol 2009 Jul;43(4):321-329.

68. Petersen RC, Doody R, Kurz A, et al. Current Concepts in Mild Cognitive Impairment 17. Arch Neurol 2001;58(12):1985-1992.

69. Blennow K, Wallin A, Hager O. Low frequency of post-lumbar puncture headache in demented patients. Acta Neurol Scand 1993 Sep;88(3):221-223.

70. Verwey NA, van der Flier WM, Blennow K, et al. A worldwide multicentre comparison of assays for cerebrospinal fluid biomarkers in Alzheimer's disease. Ann Clin Biochem 2009 May;46(Pt 3):235240. 


\section{CHAPTER 3}

TEST SEQUENCE OF CSF AND MRI BIOMARKERS FOR

PREDICTION OF AD IN SUBJECTS WITH MCI

Neurobiology of Aging 2012;33:2272-81

Stephanie Vos, Ineke van Rossum, Leah Burns, Dirk Knol, Philip Scheltens, Hilkka Soininen, Lars-Olof Wahlund, Harald Hampel, Magda Tsolaki, Lennart Minthon, Ron Handels, Gilbert L'Italien, Wiesje van der Flier, Pauline Aalten, Charlotte Teunissen, Frederik Barkhof, Kaj Blennow, Robin Wolz, Daniel Rueckert, Frans Verhey, Pieter Jelle Visser 


\section{ABSTRACT}

\section{Objective}

Our aim was to identify the best diagnostic test sequence for predicting Alzheimer's disease (AD)-type dementia in subjects with mild cognitive impairment (MCI) using cerebrospinal fluid (CSF) and MRI biomarkers.

\section{Methods}

We selected 153 subjects with MCI from a multicenter memory-clinic-based cohort. We tested the CSF A $\beta 1-42 /$ tau ratio using ELISA and hippocampal volumes (HCVs) using the atlas-based LEAP method. Outcome measure was progression to AD-type dementia in 2 years.

\section{Results}

At follow-up, 48 (31\%) subjects converted to AD-type dementia. In multivariable analyses, CSF A $\beta 1-42 /$ tau and HCV predicted AD-type dementia regardless of APOE genotype and cognitive scores. Test sequence analyses showed that CSF A $\beta 1-42 /$ tau increased predictive accuracy in subjects with normal HCV $(p<0.001)$ and abnormal HCV $(p=0.025)$. HCV increased predictive accuracy only in subjects with normal CSF A $\beta 1-42 / \operatorname{tau}(p=0.014)$. Slope analyses for annual cognitive decline yielded similar results. For selection of subjects for a prodromal AD trial, the best balance between sample size and number of subjects needed to screen was obtained with CSF markers.

\section{Conclusions}

These results provide further support for the use of CSF and MRI biomarkers to identify prodromal AD. 


\section{INTRODUCTION}

Diagnosis of Alzheimer's disease (AD) in the stage of mild cognitive impairment (MCI) is important in order to give patients a prognosis. Moreover, disease-modifying drugs for AD might be most effective in this early course of the disease. Several biomarkers of AD are now available that can help to identify AD pathology including abnormal levels of beta amyloid $(\mathrm{A} \beta) 1-42$ and tau in cerebrospinal fluid $(\mathrm{CSF})^{1-5}$ and medial temporal lobe atrophy on magnetic resonance imaging (MRI). ${ }^{6-9}$

For clinical practice and for selection of subjects for prodromal AD trials, it is of major importance to investigate which combination of CSF and MRI biomarkers could best predict AD-type dementia in subjects with MCI. Some studies found that CSF biomarkers could best predict AD-type dementia, ${ }^{10-13}$ but others studies found that MRI biomarkers were the best predictor. $^{14,15}$ Moreover, several studies showed that a combination of these biomarkers could predict AD-type dementia in subjects with MCI more accurately than each biomarker alone. ${ }^{10-12,14-16}$

These previous studies, however, had several limitations. They had a small sample size or were performed in a highly selected research sample. 10-12,14-16 Moreover, some studies included only subjects with aMCI while subjects with MCI due to AD may also present as non-amnestic MCI (naMCI). ${ }^{17}$ The use of a broad definition of MCI is also recommended in the new criteria for MCI due to AD of the National Institute on Aging and Alzheimer's Association workgroup. ${ }^{18}$

Our aim was to identify the best combination of CSF and MRI biomarkers to predict conversion to AD-type dementia after 2 years in a large clinically representative sample of subjects with MCI, such that the number of tests could be reduced and as a consequence reduce patients' burden and costs. ${ }^{19}$ We performed test sequence analyses of CSF and MRI measures and examined how the use of different combinations of biomarkers as inclusion criterion influenced the sample size and number of subjects required for screening for a fictive trial in prodromal AD. Although conversion to AD-type dementia after a 2-year follow-up is a clinically relevant outcome, the potential of CSF and MRI biomarkers to identify prodromal AD may be underestimated as subjects may convert at longer follow-up intervals. Therefore, we additionally performed slope analyses with annual cognitive decline as outcome measure. ${ }^{20}$

\section{METHODS}

\section{Subjects}

Subjects were recruited from the DESCRIPA cohort and the Alzheimer Center of the VU University medical center (VUmc) cohort. For the present study, inclusion criteria were baseline diagnosis of MCI, age $\geq 55$ years, availability of data on the CSF ratio of A $\beta 1-42$ to total tau (t-tau) and automatically measured hippocampal volumes (HCVs) on MRI, and being newly referred for assessment of cognitive complaints. Exclusion criteria were 
diagnosis of dementia at baseline or any other somatic, psychiatric or neurological disorder that might have caused the cognitive impairment.

DESCRIPA is a European multicenter study performed in a memory clinic setting. ${ }^{21}$ Subjects were recruited from 6 centers, as both MRI and CSF data were only available at these centers. Of the 207 eligible subjects enrolled between 2003 and 2005 at these centers, 74 had data for both measures. The VUmc center was one of the DESCRIPA centers and contributed an additional sample of subjects that were seen outside the DESCRIPA inclusion period. Of the 217 additional eligible subjects that were included between 1998 and 2007 at this center, 91 had baseline data for both MRI and CSF.

Subjects for whom both MRI and CSF data were available differed from subjects for whom MRI and CSF data were not both available with regard to age (69.3 vs. 71.2 years; p $<0.001$ ), MMSE score (26.6 vs. 27, p=0.019), and MCI type (68 vs. $59 \%$ aMCI; $p=0.016$ ) at baseline. The CSF A $\beta 1-42$ /tau ratio and HCV were similar in both groups. The total sample size was 165 . The medical ethics committee at each center approved the study. All patients provided informed consent.

\section{Clinical and cognitive assessment}

Assessment of the subjects included a clinical history, medical and neurological examination, laboratory tests, functional evaluation with the clinical dementia rating scale, rating scales for neuropsychiatric symptoms, scoring on the MMSE, and neuropsychological assessment. ${ }^{10,21}$ All data were collected by investigators who were blinded to the results of biomarker analyses. Follow-up assessment was performed annually up to 5 years. Primary outcome measure was conversion to AD-type dementia after 2 years according to the DSMIV $^{22}$ and NINCDS-ADRDA criteria. ${ }^{23}$ Secondary outcome measure was annual cognitive decline on the MMSE and a cognitive composite score.

Baseline diagnosis of MCI was made according to the criteria of Petersen et al. ${ }^{24}$ Raw scores on neuropsychological tests were corrected for age, education, and sex, in accordance with locally collected or published normative data and expressed as z-scores; the z-scores were used for further analysis. ${ }^{21}$ Subjects with a z-score $<-1.5$ SD on the learning measure or delayed recall of a word list learning test or equivalent memory test were classified as having aMCI $(n=102)$. Subjects with a z-score $<-1.5$ SD on the trail making test (TMT) part A, TMT part B, verbal fluency, Rey figure copy test or equivalent test but no memory impairment were classified as having naMCI $\left(n=48\right.$; see appendix S1). ${ }^{21}$ The MCI subtype diagnosis was missing for 3 subjects, as they did not undergo a neuropsychological assessment. We calculated a cognitive composite score as the average z-score of 5 tests (learning and delayed recall score of the word learning list, TMT A and B, verbal fluency, copy figures), if scores were available for at least 3 cognitive tests. ${ }^{17}$

\section{CSF analyses}

CSF was collected by lumbar puncture, centrifuged, and stored at $-80^{\circ} \mathrm{C}$ in polypropylene tubes. One sample was thawed twice but analyses without this sample revealed similar 
results. CSF A $\beta 1-42$ and t-tau were measured using commercially available sandwich ELISAs (Innotest $\beta$-amyloid 1-42; Innotest hTAU-Ag; Innogenetics, Ghent, Belgium), specially constructed to measure $A \beta 1-42$ and $t-t^{25,26}$ by experienced technicians at the lab in Gothenburg for the DESCRIPA cohort and in Amsterdam for the VUmc cohort. We corrected for interlaboratory ELISA differences by analyzing 33 samples at both labs and we adjusted VUmc values to those of DESCRIPA using the following formula: Gothenborg=(SD Gothenborg/SD VUmc)*VUmc+average Gothenborg-((SD Gothenborg /SD VUmc)*average VUmc). ${ }^{27}$

As CSF measure, we used the ratio of A $\beta 1-42$ to t-tau, which is the best CSF biomarker according to a recent meta-analysis ${ }^{28}$ and accepted by the European Medical Agency for inclusion of subjects for prodromal AD trials. ${ }^{29}$ The CSF ratio was dichotomized by defining a cut-off point (1.14) that could best predict AD-type dementia after 2 years based on the Youden index from a time-dependent ROC curve in $\mathrm{R},{ }^{30}$ including all subjects with CSF data in both cohorts ( $\mathrm{n}=219)$. In 5 subjects, CSF was obtained at follow-up. As these subjects had a normal CSF ratio score, they were considered to have a normal CSF score at baseline as well.

\section{MRI analyses}

For the DESCRIPA cohort, subjects were scanned according to the routine MRI protocol at each site (appendix S2). Scanning was performed at 1.0 or $1.5 \mathrm{~T}$ and included a threedimensional T1-weighted gradient echo sequence and a fast fluid attenuated inversion recovery (FLAIR) sequence. ${ }^{31}$

As measure of medial temporal lobe atrophy, we used HCV measured centrally at the Department of Computing at Imperial College London by experienced technicians, using LEAP. ${ }^{32}$ LEAP is an automated structural segmentation, performed by propagating multiple manually segmented atlas images to a diverse image set in a stepwise fashion, ensuring that image registration needed to be performed between similar images only. All labels propagated to a target image were considered together with a structures intensity distribution to estimate the final segmentation (e.g. supplemental figure S1). Quality control was performed by visual inspection of the segmentation on transverse, coronal and sagittal slices. HCVs were normalized to intracranial volume using MNI scaling. The sum of left and right HCV was dichotomized by defining a cut-off $\left(5.3 \mathrm{~cm}^{3}\right)$ that could best predict AD-type dementia after 2 years based on the Youden index from a time-dependent ROC curve in $\mathrm{R},{ }^{33}$ including all subjects with HCV data in both cohorts $(n=339)$. In 6 subjects, MRI was performed at follow-up. As these subjects had a normal HCV score, they were considered to have a normal HCV score at baseline as well.

\section{APOE genotype}

APOE genotype was determined by polymerase chain reaction of genomic DNA extracted from EDTA anticoagulated blood in 138 subjects. Subjects were classified as APOE- 44 carriers or non-carriers. 


\section{Statistical analyses}

Statistical analyses were done with SPSS version 16.0 (Chicago, IL, USA) and statistical software package $\mathrm{R}$ version 2.10.1.33 Significance was set at $\mathrm{p}<0.05$ and $95 \%$ confidence intervals (CI) were calculated. Differences between groups were analyzed using a t-test for continuous variables and chi-square test for categorical variables.

Univariable and multivariable logistic regression analyses were performed for dichotomized CSF and MRI markers to assess whether a combination of markers was better predictive for AD-type dementia. Sensitivity, specificity, positive predictive value, negative predictive value, Youden index (sensitivity+specificity-1) and odds ratio (OR) were calculated. Multivariable analyses with correction for center yielded similar findings. Therefore, we did not correct for center in the final analyses.

Change on the MMSE and cognitive composite score were assessed by use of slope analyses with mixed models. The analyses included the baseline score and available followup scores up to 5 years after baseline. We used an unstructured covariance structure with center as a random effect as this model provided the best -2 log likelihood compared to models with simpler covariance structures. ${ }^{17}$ We examined whether the slopes of cognition were different for subjects with and without abnormal CSF or MRI biomarker scores and whether the combination of both biomarkers increased predictive accuracy for cognitive decline.

Test sequence analyses were performed to investigate the added predictive value of a second biomarker for AD-type dementia or cognitive decline in specific subgroups. We identified the best test sequence of CSF $A \beta 1-42 /$ tau ratio and HCV assessment and visualized it using probability-modifying plots. ${ }^{34}$

The relation between biomarkers, the sample size, and number of subjects required for screening, was tested for a fictive placebo-controlled AD drug trial targeting amyloid pathology in subjects with MCI. The number of subjects was calculated in such a way that the study could detect a relative decrease of $25 \%$ in conversion rate to AD-type dementia over a 2-year period in subjects with AD pathology, which was defined as an abnormal CSF A $\beta 1-42 /$ tau ratio, with a power of $90 \%$, a 2 -sided alpha of $5 \%$, a drop-out rate of $30 \%$ and equally sized treatment groups. ${ }^{28}$ The prevalence of abnormal biomarkers and conversion rate were based on the observed data in our study.

\section{RESULTS}

\section{Sample characteristics}

153 subjects had at least one follow-up assessment (average 3.7 years ( $\mathrm{SD}=1.4)$ ). $48(31 \%)$ subjects converted to AD-type dementia after 2 years, 6 (4\%) subjects converted to other types of dementia and were included in the non-AD group. Subjects with at least one followup had similar baseline characteristics as those without follow-up (supplemental table S1). 


\section{Predictors of AD-type dementia}

\section{Univariable predictors}

Subjects with AD-type dementia at 2-year follow-up had lower MMSE scores ( $p=0.014)$, lower cognitive composite score $(p=0.009)$, lower CSF levels of $A \beta 1-42(p<0.001)$, higher CSF levels of t-tau $(p<0.001)$, a lower CSF A $\beta 1-42 /$ tau ratio $(p<0.001)$, smaller HCV $(\mathrm{p}<0.001)$, a higher frequency of APOE- $\varepsilon 4$ alleles $(\mathrm{p}=0.011)$, and tended to have more often aMCI (75 vs. $65 \%, p>0.10$ ), compared to subjects without AD-type dementia (table 1). Scores on verbal fluency $(p=0.052)$ and delayed recall $(p=0.069)$ tended to be lower for subjects who progressed to AD-type dementia compared to those who did not progress. The overall predictive accuracy (OR) was more than twice as high for the CSF A $\beta 1-42 /$ tau ratio (OR=9.2, 95\% CI 3.9-22, p<0.001) than for HCV (OR=3.7, 95\% CI 1.8-7.6, p<0.001, table 2). Of the subjects without dementia at follow-up, 14 had a follow-up shorter than 1.5 years. When we repeated analyses after exclusion of these subjects, results were similar.

Table 1. Baseline characteristics of subjects with MCI by outcome at 2-year follow-up

\begin{tabular}{lll}
\hline & MCI-non AD & MCI-AD \\
\hline $\mathrm{N}^{\ddagger}$ & 105 & 48 \\
Age & $68.8(7.3)$ & $70.4(7.2)$ \\
Female, n (\%) & $43(41.0)$ & $24(50.0)$ \\
aMCI, n (\%) & $67(65.0)$ & $35(74.5)$ \\
Years of education & $10.7(3.2)$ & $11.2(3.0)$ \\
MMSE & $26.9(2.6)$ & $25.8(2.8)^{*}$ \\
Delayed recall, z-score & $-1.34(1.1)$ & $-1.76(1.0)$ \\
Learning, z-score & $-1.15(1.1)$ & $-1.44(1.1)$ \\
Verbal fluency, z-score & $-0.66(1.1)$ & $-1.04(0.9)$ \\
TMT A, z-score & $-0.44(1.6)$ & $-0.87(1.8)$ \\
TMT B, z-score & $-0.60(1.3)$ & $-0.85(1.3)$ \\
Visuo-construction, z-score & $0.26(1.2)$ & $0.18(1.2)$ \\
Cognitive composite score, z-score & $-0.63(0.7)$ & $-0.95(0.7)^{\dagger}$ \\
APOE $\varepsilon 4$ carrier, n (\%) & $42(44.2)$ & $29(67.4)^{*}$ \\
Hippocampal volume, cm ${ }^{3}$ & $5.7(0.7)$ & $5.3(0.6)^{\ddagger}$ \\
A $\beta 1-42$, pg/ml & $682(309)$ & $470(145)^{\ddagger}$ \\
T-tau, pg/ml & $409(219)$ & $686(377)^{\ddagger}$ \\
Ratio A $\beta 1-42 / t$-tau & $2.3(1.7)$ & $0.8(0.5)^{\ddagger}$ \\
\hline
\end{tabular}

Data are mean (SD), unless specified otherwise. ${ }^{*} \mathrm{P}<0.05 ;{ }^{\dagger} \mathrm{p}<0.01 ;{ }^{\ddagger} \mathrm{p}<0.001$ compared to MCI-non AD. Group size is different for cognitive tests, APOE, hippocampal volume, and CSF measures. MCI-nonAD=subjects with MCI who did not convert to AD-type dementia at follow-up; $\mathrm{MCI}-\mathrm{AD}=$ subjects with $\mathrm{MCI}$ who converted to AD-type dementia at follow-up; aMCI=amnestic mild cognitive impairment; MMSE=Mini-Mental State Examination; TMT=trail making test; APOE=apolipoprotein E genotype; $A \beta 1-42=$ beta amyloid 1-42; $\mathrm{t}$-tau=total tau.

\section{Multivariable predictors}

When both the CSF A $\beta 1-42 /$ tau ratio and HCV were entered in the same model, the markers predicted AD-type dementia independently from each other (OR CSF ratio=7.9, 95\% CI 3.3- 
19, $\mathrm{p}<0.001 ; \mathrm{OR} \mathrm{HCV}=2.8,95 \%$ CI 1.3-6.2, $\mathrm{p}=0.01$ ). The combination increased the predictive accuracy relative to the model with only the CSF $A \beta 1-42 /$ tau ratio $\left(\chi^{2}=6.6, d f=1\right.$, $\mathrm{p}=0.01$ ). Next, we tested the added diagnostic value of CSF $A \beta 1-42 /$ tau ratio and $\mathrm{HCV}$ compared to models with other markers that could predict AD-type dementia in the univariable analyses and age. When age, MMSE score, and APOE genotype were entered in the first step, CSF A $\beta 1-42 /$ tau ratio and HCV increased the predictive accuracy in the second step $\left(\chi^{2}=24.1, \mathrm{df}=2, \mathrm{p}<0.001\right)$. Both markers were significant predictors (OR CSF ratio=7.9, 95\% CI 2.8-22, p<0.001; OR HCV=2.8, 95\% CI 1.1-6.9, p=0.03), whereas age, MMSE score, and APOE genotype no longer predicted outcome ( $p>0.42)$. When we tested a multivariable model in which the cognitive composite score was entered in the first step $(p=0.012)$ and CSF A $\beta 1-42 /$ tau ratio and HCV in the second step, both biomarkers predicted AD-type dementia $(p<0.05)$ but the cognitive composite score not $(p=0.13)$.

Table 2. Predictive accuracy of CSF and MRI markers for AD-type dementia after 2 years

\begin{tabular}{lllllll}
\hline Markers & Sensitivity & Specificity & PPV & NPV & $\begin{array}{l}\text { Youden } \\
\text { Index }\end{array}$ & $\begin{array}{l}\text { Odds } \\
\text { ratio }^{*}\end{array}$ \\
\hline CSF A $\beta 1-42 /$ tau & 0.83 & 0.65 & 0.52 & 0.89 & 0.48 & 9.2 \\
& $(0.73-0.94)$ & $(0.56-0.74)$ & $(0.41-0.63)$ & $(0.83-0.96)$ & $(0.34-0.62)$ & $(3.9-22)$ \\
HCV & 0.56 & 0.74 & 0.50 & 0.79 & 0.31 & 3.7 \\
& $(0.42-0.70)$ & $(0.66-0.83)$ & $(0.37-0.63)$ & $(0.71-0.87)$ & $(0.14-0.47)$ & $(1.8-7.6)$ \\
CSF A $\beta 1-42 /$ tau & 0.94 & 0.52 & 0.47 & 0.95 & 0.46 & 17 \\
or HCV abnormal & $(0.87-1.00)$ & $(0.43-0.62)$ & $(0.37-0.57)$ & $(0.89-1.00)$ & $(0.34-0.58)$ & $(4.8-56)$ \\
CSF A $\beta 1-42 /$ tau & 0.46 & 0.87 & 0.61 & 0.78 & 0.33 & 5.5 \\
and HCV abnormal & $(0.32-0.60)$ & $(0.80-0.93)$ & $(0.45-0.77)$ & $(0.70-0.85)$ & $(0.17-0.48)$ & $(2.5-12)$ \\
\hline
\end{tabular}

*All p-values $<0.001$. Results are presented with $95 \%$ confidence intervals.

Youden index=(sensitivity+specificity-1). CSF=cerebrospinal fluid; MRI=magnetic resonance imaging; $\mathrm{MCI}=\mathrm{mild}$ cognitive impairment; HCV=hippocampal volume; PPV=positive predictive value; NPV=negative predictive value.

\section{Test sequence}

In further analyses, we only included the CSF and HCV measures because other covariates did not contribute to the overall predictive accuracy.The added predictive value of the CSF $\mathrm{A} \beta 1-42 /$ tau ratio and HCV to the other marker is presented in supplemental S2 and figure 1. If the CSF A $\beta 1-42 /$ tau ratio was analyzed first, assessment of HCV increased predictive accuracy for AD-type dementia in subjects with a normal CSF $A \beta 1-42 /$ tau ratio (OR=7.1, $\mathrm{p}=0.014$ ), but not in subjects with an abnormal CSF $A \beta 1-42 /$ tau ratio $(\mathrm{OR}=2.0, \mathrm{p}=0.13)$. If HCV was analyzed first, assessment of CSF A $\beta 1-42 /$ tau ratio increased predictive accuracy both in subjects with normal HCV (OR=14.4, p<0.001) and with abnormal HCV (OR=4.1, $\mathrm{p}=0.025)$. Figure 1 shows probability plots of $A D$-type dementia for different sequences of CSF A $\beta 1-42 /$ tau ratio and HCV assessment, thereby visualizing the change in AD probability after adding the results for each biomarker. 
A

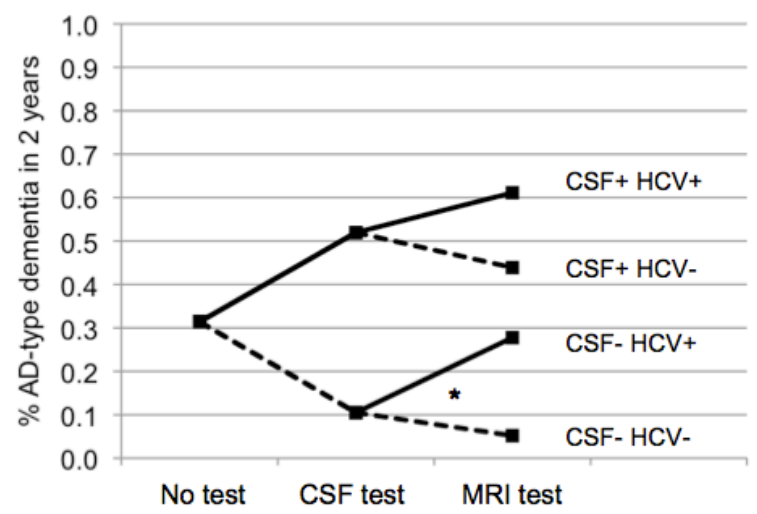

B

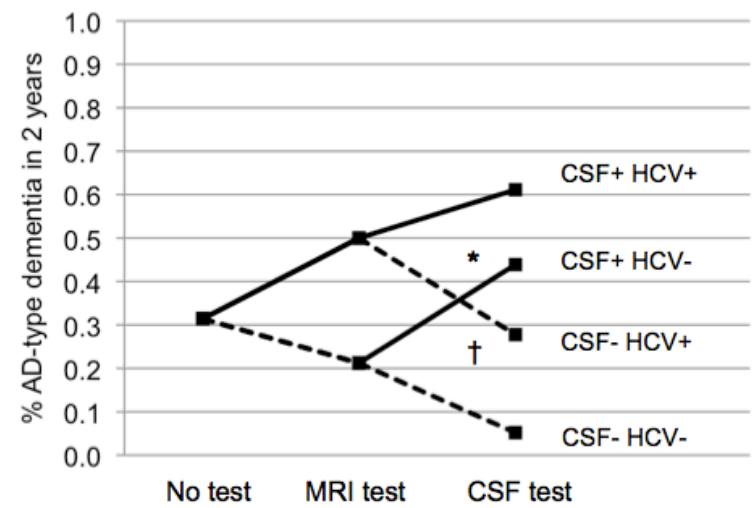

Figure 1. Probability of AD-type dementia in 2 years related to the sequence of CSF and HCV assessment.

The graph shows the change in $\mathrm{AD}$ probability according to biomarker scores. The starting point represents the $\mathrm{AD}$ conversion rate in the total MCI population. Dotted lines represent negative (normal) test results and full lines represent positive (abnormal) test results. (A) First CSF assessment then HCV assessment; (B) First HCV assessment then CSF assessment. 36 subjects had both and abnormal CSF ratio and HCV (with 22 converters to AD-type dementia), 41 subjects had an abnormal CSF ratio and a normal HCV (18 converters), 18 subjects had a normal CSF ratio and an abnormal HCV (5 converters), 58 subjects had both a normal CSF ratio and HCV (3 converters). $\mathrm{AD}=$ Alzheimer's disease; $\mathrm{CSF}=$ cerebrospinal fluid; $\mathrm{HCV}=$ hippocampal volume. ${ }^{*} \mathrm{P}<0.05$; ${ }^{\dagger} \mathrm{p}<0.001$ : difference in $\mathrm{AD}$ probability in 2 years between subjects with normal and abnormal biomarkers after second biomarker assessment.

\section{Predictors of cognitive decline}

Although conversion to AD-type dementia after 2-year follow-up is a clinically relevant outcome measure, the potential of CSF and MRI biomarkers to identify prodromal AD may be underestimated as subjects may convert at longer follow-up intervals. Therefore, we additionally performed slope analyses for annual cognitive decline over the 5-year followup period.

\section{Univariable predictors}

The CSF Ab1-42/tau ratio at baseline predicted cognitive decline on the MMSE and cognitive composite score (all $\mathrm{p}<0.001)$, while HCV only predicted decline on the MMSE $(\mathrm{p}<0.008$; table 3). The CSF Ab1-42/tau ratio was a better predictor for decline on the MMSE compared to HCV (difference $-2 \log$ likelihood (LL)=15.4; CSF F=27.6, p<0.001; MRI F=7.5, $\mathrm{p}=0.008)$.

\section{Multivariable predictors}

Multivariable analyses were performed with decline on the MMSE as outcome, since only for this outcome measure both biomarkers predicted decline in univariable analyses. When the CSF Ab1-42/tau ratio was entered first, addition of HCV significantly increased the overall predictive accuracy (decrease in $-2 \mathrm{LL}=6.5, \mathrm{~F}=4.72$, $\mathrm{p}=0.011$ ).

\section{Test sequence}

The added predictive value for decline on the MMSE of the CSF A $\beta 1-42 /$ tau ratio and HCV to the other marker is presented in supplemental table S3 and figure 2. If the CSF A $\beta 1-42 /$ tau 
ratio was analyzed first, assessment of HCV increased predictive accuracy for cognitive decline in subjects with a normal CSF $A \beta 1-42 /$ tau ratio $(p=0.001)$, but not in subjects with an abnormal CSF A $\beta 1-42$ /tau ratio ( $p>0.3$ ). If HCV was analyzed first, assessment of CSF A $\beta 1-42$ /tau ratio increased predictive accuracy both in subjects with normal HCV $(\mathrm{p}<0.001)$ and with abnormal HCV ( $\mathrm{p}=0.038)$.

Table 3. Annual cognitive decline over 5 years of follow-up according to CSF and MRI biomarker results at baseline

\begin{tabular}{lllllll}
\hline & & \multicolumn{2}{l}{ MMSE } & & \multicolumn{2}{l}{ Cognitive composite score } \\
\cline { 3 - 4 } \cline { 5 - 6 } & & Baseline & Slope & & Baseline score & Slope \\
& score & & & \\
\hline CSF A $\beta 1-42 /$ tau & Normal & $27.5(0.82)^{\S}$ & $-0.28^{*} \neq(0.13)$ & & $-0.70(0.10)^{\S}$ & $0.001^{\ddagger}(0.02)$ \\
& Abnormal & $26.6(0.83)$ & $-1.24^{\dagger}(0.12)$ & & $-0.93(0.11)$ & $-0.25^{\dagger}(0.04)$ \\
HCV & Normal & $27.3(0.73)^{¥}$ & $-0.61^{\dagger ¥}(0.13)$ & & $-0.76(0.09)$ & $-0.05(0.02)$ \\
& Abnormal & $26.1(0.80)$ & $-1.17^{\dagger}(0.16)$ & & $-0.89(0.11)$ & $-0.14^{*}(0.04)$ \\
\hline
\end{tabular}

Data are mean (SE). Slope refers to annual change on the test. A negative slope indicates cognitive decline. $\mathrm{CSF}=$ Cerebrospinal fluid, HCV=Hippocampal volume, MMSE=Mini Mental State Examination. ${ }^{*} \mathrm{P}<0.05,{ }^{\dagger} \mathrm{p}<0.001 \mathrm{for}$ slope different from zero (this means a statistically significant change over time in test score); $¥ \mathrm{P}<0.001,{ }^{\sharp} \mathrm{p}<0.01$, $\S p<0.05$ for baseline score or slope compared to baseline score or slope in abnormal biomarker group.

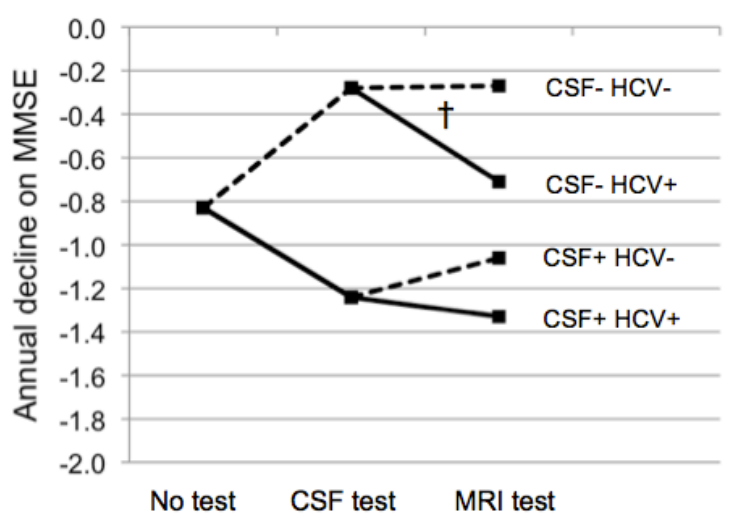

B

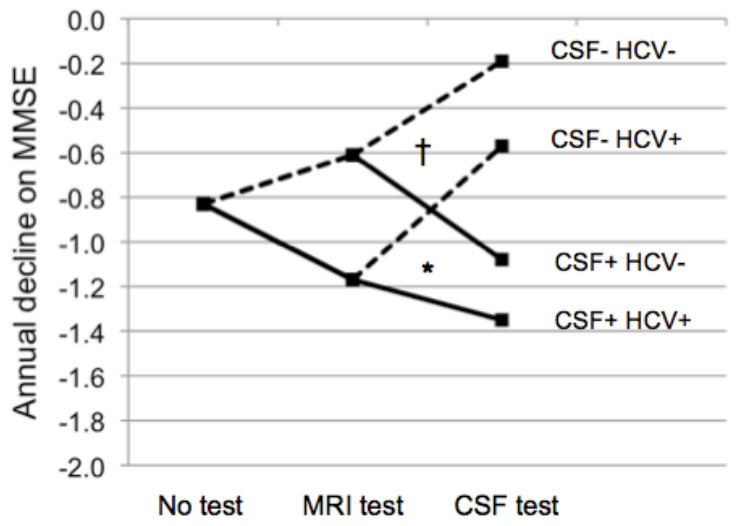

Figure 2. Average annual cognitive decline on MMSE related to the sequence of CSF and HCV assessment.

The graph shows change in annual cognitive decline according to biomarker scores. The starting point represents the decline on MMSE in the total MCI population regardless of biomarker results. Dotted lines represent negative (normal) test results and full lines represent positive (abnormal) test results. (A) First CSF assessment then HCV assessment; (B) First HCV assessment then CSF assessment. MMSE=Mini Mental State Examination; $\mathrm{CSF}=$ cerebrospinal fluid; HCV=hippocampal volume. As the sample size of the subgroups was different, different slopes may be presented for the same final groups. ${ }^{*} \mathrm{P}<0.05$; ${ }^{\dagger} \mathrm{p}<0.001$ : difference in annual cognitive decline on MMSE between subjects with normal and abnormal biomarkers after second biomarker assessment. 


\section{Biomarkers as inclusion criterion for prodromal AD trial}

We used the different combinations of the CSF A $\beta 1-42 /$ tau ratio and HCV shown in table 2 to select subjects with MCI for a fictive amyloid targeting AD trial with conversion to ADtype dementia after 2 years as outcome. The sample sizes, numbers required for screening, and screening failure rates varied considerably between the different combinations (table 4). The sample size was smallest for the strategy that required both an abnormal CSF A $\beta 1$ 42 /tau ratio and abnormal HCV at baseline (642 vs. 880-1381). The number required for screening was lowest if only the CSF A $\beta 1-42 /$ tau ratio was required to be abnormal (1761 vs. 2228-3931). The screening failure rate was lowest if either the CSF A $\beta 1-42 /$ tau ratio or HCV was required to be abnormal (38\% vs. 50-76\%). The strategy that required only abnormal CSF markers had the best trade-off between sample size $(n=880)$ and number required for screening ( $\mathrm{n}=1761)$, as numbers were best (screening) or second best (sample size).

Table 4. Number of subjects required for AD trial design based on biomarker inclusion criteria

\begin{tabular}{lccc}
\hline Biomarkers & $\begin{array}{c}\text { Number required for } \\
\text { screening }\end{array}$ & $\begin{array}{c}\text { Sample size } \\
\text { for trial }\end{array}$ & $\begin{array}{c}\text { Screening } \\
\text { failure rate }\end{array}$ \\
\hline CSF A $\beta 1-42$ tau abnormal & 1761 & 880 & 0.50 \\
HCV abnormal & 3931 & 1376 & 0.65 \\
CSF A $\beta 1-42 /$ tau or HCV abnormal & 2228 & 1381 & 0.38 \\
CSF A $\beta 1-42 /$ tau and HCV & 2675 & 642 & 0.76 \\
abnormal & & & \\
\hline
\end{tabular}

The assumptions with regard to trial design are described in the methods section. Screening failure rate=1-sample size divided by number required for screening, i.e. the proportion of subjects that were screened but not included in the AD trial, as they did not have abnormal biomarkers. Note that this failure rate does not take into account failure rates of $\mathrm{CSF}$ collection. $\mathrm{AD}=$ Alzheimer's disease; $\mathrm{CSF}=$ cerebrospinal fluid; $\mathrm{HCV}=$ hippocampal volume.

\section{DISCUSSION}

We showed that the CSF $A \beta 1-42 /$ tau ratio was the best predictor for AD-type dementia at follow-up and for cognitive decline in subjects with MCI. HCV could increase the predictive accuracy of the CSF $A \beta 1-42 /$ tau ratio. This increase in predictive accuracy for AD-type dementia and cognitive decline was because HCV predicted AD-type dementia and cognitive decline in subjects with a normal CSF A $\beta 1-42 /$ tau ratio.

Test sequence analyses suggested that CSF assessment should be performed first for prediction of AD-type dementia at follow-up. HCV assessment may be considered in subjects who have a normal CSF A $\beta 1-42 /$ tau ratio. A strategy in which HCV assessment is performed first and CSF assessment next seems less efficient as CSF assessment would further increase predictive accuracy regardless of the outcome of the HCV analysis. However, with respect to feasibility, MRI scanning may be more acceptable than CSF collection in daily practice. MRI 
is often already routinely done to exclude other diseases. Still, volumetric assessment of the hippocampus is not widely available.

Furthermore, the current study showed that for a fictive AD trial targeting amyloid pathology in subjects with MCI, subjects could be best selected based on CSF biomarkers because this provided the best balance between sample size and number of subjects required for screening. Still, the sample size was smaller if both CSF A $\beta 1-42 /$ tau ratio and HCV were required to be abnormal. However, the screening failure rate was very high for this combination, which may limit its clinical utility. The screening failure rate was lowest if subjects were required to have either an abnormal CSF A $\beta 1-42 /$ tau ratio or an abnormal $\mathrm{HCV}$. A disadvantage of this approach was the much higher sample size compared to that of the selection based on an abnormal CSF A $\beta 1-42 /$ tau ratio alone. Selection based on an abnormal HCV alone was least efficient because both the number required for screening and sample size were among the highest of the four approaches. This may be partly because the fictive trial was designed for targeting amyloid pathology such that only subjects with an abnormal CSF A $\beta 1-42 /$ tau ratio were supposed to respond to therapy.

This is the first large-scale multicenter study conducted in a memory clinic setting of subjects classified according to a broad definition of MCI, including aMCI (68\%) and naMCI that compared CSF and MRI markers for prediction of cognitive decline. Our study corroborates the findings of studies of subjects with aMCI, ${ }^{14-16}$ that the combination of the CSF ratio and measure of medial temporal lobe atrophy predicted AD-type dementia better than each biomarker alone. This also confirms the findings of smaller single-center studies performed in subjects with MCI. ${ }^{10-12}$ It validates the use of biomarkers for prediction of ADtype dementia in subjects who meet the criteria of a broad definition of $\mathrm{MCI}$, including aMCI and naMCI. This supports the new NIA-AA criteria for MCI due to AD, which uses a broad definition of MCI as well. ${ }^{18}$ The conversion rate of $31 \%$ to AD-type dementia after 2 years in the present study is comparable to conversion rates in previous studies with a similar follow-up period. ${ }^{11,12,15}$

The overall predictive accuracy (OR) for AD-type dementia of the CSF A $\beta 1-42 /$ tau ratio was twice as high as that of the HCV. This was mainly due to the higher sensitivity of the CSF A $\beta 1-42 /$ tau ratio compared to that of the HCV (table 2). This is in line with the hypothetical dynamic model of AD biomarkers, ${ }^{35}$ stating that CSF biomarkers become abnormal at an earlier stage than MRI biomarkers. We also found that the specificity of the HCV was higher than that of the CSF A $\beta 1-42 /$ tau ratio. This may be considered unexpected as medial temporal lobe atrophy is not specific for $\mathrm{AD}$ and also observed in other neurodegenerative and vascular disorders. However, the higher specificity of the HCV could also be explained by the hypothetical dynamic model of AD biomarkers. As CSF markers are expected to become abnormal before the $\mathrm{HCV},{ }^{35}$ there will be more MCI subjects with prodromal $\mathrm{AD}$ with an abnormal CSF $\mathrm{A} \beta 1-42 /$ tau ratio who have not yet converted to ADtype dementia within 2 years than there will be $\mathrm{MCI}$ subjects with prodromal AD with an abnormal HCV who have not yet converted to AD-type dementia within 2 years. 
Test sequence analyses revealed the presence of a small group of subjects ( $n=18,12 \%$ of the total sample) with a normal CSF A $\beta 1-42$ /tau ratio but abnormal HCV and a conversion rate of $28 \%$ to AD-type dementia after 2 years. This is an interesting group because it is not in line with the hypothetical dynamic model of AD biomarkers, ${ }^{35}$ as abnormal CSF biomarkers did not precede medial temporal lobe atrophy. Further research is needed to investigate whether these subjects have an atypical presentation of $\mathrm{AD}$ or whether they are misclassified cases either because of the applied cut-offs or because AD is not the cause of the dementia.

MMSE score, a cognitive composite score, and APOE genotype predicted AD-type dementia in univariable analyses, however, in the combined model with CSF and MRI markers, these variables were no longer significant predictors. This indicates that CSF and MRI can be used independent of cognitive scores and APOE genotype for short-term prediction of AD-type dementia. Nevertheless, our findings are inconsistent with some previous studies of subjects with aMCI. One study showed that MRI and APOE genotype did not significantly add to the predictive accuracy of neuropsychological tests. ${ }^{36}$ Another study found that the optimal biomarker combination and neuropsychological tests were equally predictive for AD-type dementia. ${ }^{14}$ The more severely impaired cohort that was used in the former study and the inclusion of only subjects with aMCI in both studies could explain the inconsistent findings.

Although a 2-year follow-up is clinically relevant, the potential of CSF and MRI biomarkers to identify prodromal AD may be underestimated. ${ }^{20}$ Therefore, we also performed slope analyses for annual cognitive decline over 5 years. These analyses yielded very similar findings as our analysis on conversion to AD-type dementia after 2 years. The CSF A $\beta 1-42 /$ tau ratio predicted annual cognitive decline better than HCV. In the sequence analysis with decline on MMSE as outcome, HCV increased predictive accuracy only in subjects with a normal CSF A $\beta 1-42 /$ tau ratio but not in subjects with an abnormal ratio, while the CSF A $\beta 1-42$ /tau ratio improved predictive accuracy regardless of HCV. The lower predictive accuracy of HCV can again in part be explained by a lower sensitivity of this marker for prodromal AD. This is supported by the observation that decline in subjects with a normal HCV is higher than in subjects with a normal CSF ratio in table 3. This higher decline is probably driven by subjects with an abnormal CSF ratio among subjects with a normal HCV.

This study had several limitations. As the findings were based on memory clinic populations, they may not be generalized to other settings, including the general population. Furthermore, the cut-offs of the CSF A $\beta 1-42 /$ tau ratio and HCV were determined within a study population that also included the subjects from the present analyses. Although this may have led to an overestimation of the predictive accuracy of the biomarkers, it is unlikely that it influences our findings with respect to the differences in predictive accuracy between the CSF A $\beta 1-42 /$ tau ratio and HCV, as we used the same method to define the cut-point for each biomarker. We defined cut-offs regardless of age, although biomarkers may change with age. However, when we repeated analyses using age-adjusted cut-offs $(\leq 70$ vs. $>70$ 
years) analyses yielded similar findings. In addition, there was variability in scanners used, which may have influenced the volumetric measurements and predictive accuracy of the HCV. However, the LEAP approach has been shown to be robust for scanner variability. ${ }^{32}$ The follow-up period to AD-type dementia was relatively short but for clinical trials shortterm prognosis may be important. However, we used a longer follow-up period to investigate the predictive accuracy of CSF and MRI biomarkers for annual cognitive decline over 5 years. Moreover, the diagnosis of AD-type dementia at follow-up was not neuropathologically validated. This may have led to the misclassification of some cases.

A major strength of this study was the large sample size. The overall multicenter design favors generalizability to other memory clinic settings, although a considerable amount of subjects was recruited from one single center. Furthermore, this is the first study of predictive accuracy of the combination of CSF and MRI markers for AD-type dementia and cognitive decline that investigated the test sequence of CSF and HCV assessments in an unselected population with subjects classified according to a broad definition of MCI.

In sum, test sequence analyses may lead to cost reduction and to a decrease in patients' burden. Future research is needed to evaluate the ratio of costs and effectiveness for patients regarding biomarker assessment, as this may eventually require adjustments to patient policy. Also the combination of CSF and MRI biomarkers with PIB-PET, and FDG-PET should be investigated, since this may further aid to define an algorithm of markers for the accurate prediction of AD-type dementia in subjects with MCI. While hippocampal atrophy and CSF markers are given equal diagnostic accuracy in recent criteria for prodromal AD or MCI-due to AD, our findings suggest that this may not be the case and a CSF A $\beta 1-42 /$ tau ratio may be preferred over HCV assessment because of its higher sensitivity. 


\section{REFERENCES}

1. Blennow K, Hampel H. CSF markers for incipient Alzheimer's disease. Lancet Neurol 2003;2:605613.

2. Hansson O, Zetterberg H, Buchhave P, Londos E, Blennow K, Minthon L. Association between CSF biomarkers and incipient Alzheimer's disease in patients with mild cognitive impairment: a followup study. Lancet Neurol 2006;5:228-234.

3. Herukka SK, Hallikainen M, Soininen H, Pirttila T. CSF Abeta42 and tau or phosphorylated tau and prediction of progressive mild cognitive impairment. Neurology 2005;64:1294-1297.

4. Mattsson N, Zetterberg H, Hansson O, et al. CSF biomarkers and incipient Alzheimer disease in patients with mild cognitive impairment. JAMA 2009;302:385-393.

5. Shaw LM, Vanderstichele H, Knapik-Czajka M, et al. Cerebrospinal fluid biomarker signature in Alzheimer's disease neuroimaging initiative subjects. Ann Neurol 2009;65:403-413.

6. Devanand DP, Pradhaban G, Liu X, et al. Hippocampal and entorhinal atrophy in mild cognitive impairment: prediction of Alzheimer disease. Neurology 2007;68:828-836.

7. Geroldi C, Rossi R, Calvagna C, et al. Medial temporal atrophy but not memory deficit predicts progression to dementia in patients with mild cognitive impairment. J Neurol Neurosurg Psychiatry 2006;77:1219-1222.

8. Jack CR, Jr., Petersen RC, Xu YC, et al. Prediction of AD with MRI-based hippocampal volume in mild cognitive impairment. Neurology 1999;52:1397-1403.

9. Visser PJ, Verhey FR, Hofman PA, Scheltens P, Jolles J. Medial temporal lobe atrophy predicts Alzheimer's disease in patients with minor cognitive impairment. J Neurol Neurosurg Psychiatry 2002;72:491-497.

10. Bouwman FH, Schoonenboom SN, van der Flier WM, et al. CSF biomarkers and medial temporal lobe atrophy predict dementia in mild cognitive impairment. Neurobiol Aging 2007;28:1070-1074.

11. Eckerstrom C, Andreasson U, Olsson E, et al. Combination of hippocampal volume and cerebrospinal fluid biomarkers improves predictive value in mild cognitive impairment. Dement Geriatr Cogn Disord 2010;29:294-300.

12. Galluzzi S, Geroldi C, Ghidoni R, et al. The new Alzheimer's criteria in a naturalistic series of patients with mild cognitive impairment. J Neurol 2010;257:2004-2014.

13. Landau SM, Harvey D, Madison CM, et al. Comparing predictors of conversion and decline in mild cognitive impairment. Neurology 2010;75:230-238.

14. Ewers M, Walsh C, Trojanowski JQ, et al. Prediction of conversion from mild cognitive impairment to Alzheimer's disease dementia based upon biomarkers and neuropsychological test performance. Neurobiol Aging 2012;33:1203-1214.

15. Vemuri P, Wiste HJ, Weigand SD, et al. MRI and CSF biomarkers in normal, MCI, and AD subjects: predicting future clinical change. Neurology 2009;73:294-301.

16. Heister D, Brewer JB, Magda S, Blennow K, McEvoy LK. Predicting MCI outcome with clinically available MRI and CSF biomarkers. Neurology 2011;77:1619-1628.

17. Visser PJ, Verhey F, Knol DL, et al. Prevalence and prognostic value of CSF markers of Alzheimer's disease pathology in patients with subjective cognitive impairment or mild cognitive impairment in the DESCRIPA study: a prospective cohort study. Lancet Neurol 2009;8:619-627.

18. Albert MS, Dekosky ST, Dickson D, et al. The diagnosis of mild cognitive impairment due to Alzheimer's disease: Recommendations from the National Institute on Aging-Alzheimer's Association workgroups on diagnostic guidelines for Alzheimer's disease. Alzheimers Dement 2011;7:270-279. 
19. Hampel H, Wilcock G, Andrieu S, et al. Biomarkers for Alzheimer's disease therapeutic trials. Prog Neurobiol 2011;95:579-593.

20. Aisen PS, Andrieu S, Sampaio C, et al. Report of the task force on designing clinical trials in early (predementia) AD. Neurology 2011;76:280-286.

21. Visser PJ, Verhey FR, Boada M, et al. Development of screening guidelines and clinical criteria for predementia Alzheimer's disease. The DESCRIPA Study. Neuroepidemiology 2008;30:254-265.

22. American. Psychiatric Association: Diagnostic and Statistical Manual of Mental Disorders,. 4th ed. Washington DC: American Psychiatric Association, 1994.

23. McKhann G, Drachman D, Folstein M, Katzman R, Price D, Stadlan EM. Clinical diagnosis of Alzheimer's disease: report of the NINCDS-ADRDA Work Group under the auspices of Department of Health and Human Services Task Force on Alzheimer's Disease. Neurology 1984;34:939-944.

24. Petersen RC. Mild cognitive impairment as a diagnostic entity. J Intern Med 2004;256:183-194.

25. Andreasen N, Hesse C, Davidsson P, et al. Cerebrospinal fluid beta-amyloid(1-42) in Alzheimer disease: differences between early- and late-onset Alzheimer disease and stability during the course of disease. Arch Neurol 1999;56:673-680.

26. Blennow K, Wallin A, Agren H, Spenger C, Siegfried J, Vanmechelen E. Tau protein in cerebrospinal fluid: a biochemical marker for axonal degeneration in Alzheimer disease? Mol Chem Neuropathol 1995;26:231-245.

27. Kolen M BR. Test Equating: Methods and Practices. New York: Springer-Verlag, 1995.

28. van Rossum IA, Vos S, Handels R, Visser PJ. Biomarkers as predictors for conversion from mild cognitive impairment to Alzheimer-type dementia: implications for trial design. J Alzheimers Dis 2010;20:881-891.

29. Isaac M, Vamvakas S, Abadie E, Jonsson B, Gispen C, Pani L. Qualification opinion of novel methodologies in the predementia stage of Alzheimer's disease: cerebro-spinal-fluid related biomarkers for drugs affecting amyloid burden--regulatory considerations by European Medicines Agency focusing in improving benefit/risk in regulatory trials. Eur Neuropsychopharmacol 2011;21:781-788.

30. Heagerty PJ, Lumley T, Pepe MS. Time-dependent ROC curves for censored survival data and a diagnostic marker. Biometrics 2000;56:337-344.

31. van de Pol LA, Verhey F, Frisoni GB, et al. White matter hyperintensities and medial temporal lobe atrophy in clinical subtypes of mild cognitive impairment: the DESCRIPA study. J Neurol Neurosurg Psychiatry 2009;80:1069-1074.

32. Wolz R, Aljabar P, Hajnal JV, Hammers A, Rueckert D. LEAP: learning embeddings for atlas propagation. Neuroimage 2010;49:1316-1325.

33. R Development Core Team. R: A language and environment for statistical computing. Vienna, 2010.

34. Severens JL, Sonke G, Laheij RJ, Verbeek AL, De Vries Robbe PF. Efficient diagnostic test sequence: applications of the probability-modifying plot. J Clin Epidemiol 2001;54:1228-1237.

35. Jack CR, Jr., Knopman DS, Jagust WJ, et al. Hypothetical model of dynamic biomarkers of the Alzheimer's pathological cascade. Lancet Neurol 2010;9:119-128.

36. Fleisher AS, Sun S, Taylor C, et al. Volumetric MRI vs clinical predictors of Alzheimer disease in mild cognitive impairment. Neurology 2008;70:191-199. 


\section{SUPPLEMENTAL DATA}

Supplemental Table S1. Baseline characteristics for subjects with and without follow-up

\begin{tabular}{llll}
\hline & MCI all & MCI FU & MCI no FU \\
\hline $\mathrm{N}^{*}$ & 165 & 153 & 12 \\
Age & $69.3(7.3)$ & $69.3(7.3)$ & $70.3(6.8)$ \\
Female, n (\%) & $73(44.2)$ & $67(43.8)$ & $6(50.0)$ \\
aMCI, n (\%) & $112(67.9)$ & $102(66.7)$ & $10(83.3)$ \\
Years of education & $10.8(3.1)$ & $10.9(3.2)$ & $10.2(2.8)$ \\
MMSE & $26.6(2.6)$ & $26.6(2.7)$ & $26.6(2.1)$ \\
APOE $\varepsilon 4$ carrier, n (\%) & $75(50.3)$ & $71(51.4)$ & $4(36.4)$ \\
Hippocampal volume, cm ${ }^{3}$ & $5.6(0.7)$ & $5.6(0.7)$ & $5.8(0.1)$ \\
A $31-42, \mathrm{pg} / \mathrm{ml}$ & $609(282)$ & $616(285)$ & $527(236)$ \\
T-tau, pg/ml & $487(301)$ & $496(305)$ & $377(219)$ \\
Ratio A $\beta 1-42 / t$-tau & $1.9(1.6)$ & $1.8(1.6)$ & $2.0(1.6)$ \\
\hline
\end{tabular}

Data are mean (SD), unless specified otherwise. ${ }^{*}$ Group size is different for APOE, hippocampal volume and CSF measures. No significant statistical differences were found between subjects with and without follow-up. FU=followup; aMCI=amnestic mild cognitive impairment; MMSE=Mini-Mental State Examination; APOE=apolipoprotein E genotype; $A \beta 1-42=$ beta amyloid 1-42; t-tau=total tau.

\section{Supplemental Figure S1. Image of hippocampal LEAP segmentation.}

This figure shows an example of an image of hippocampal volume segmentation for horizontal, coronal, and sagittal sections using the atlas-based LEAP method.

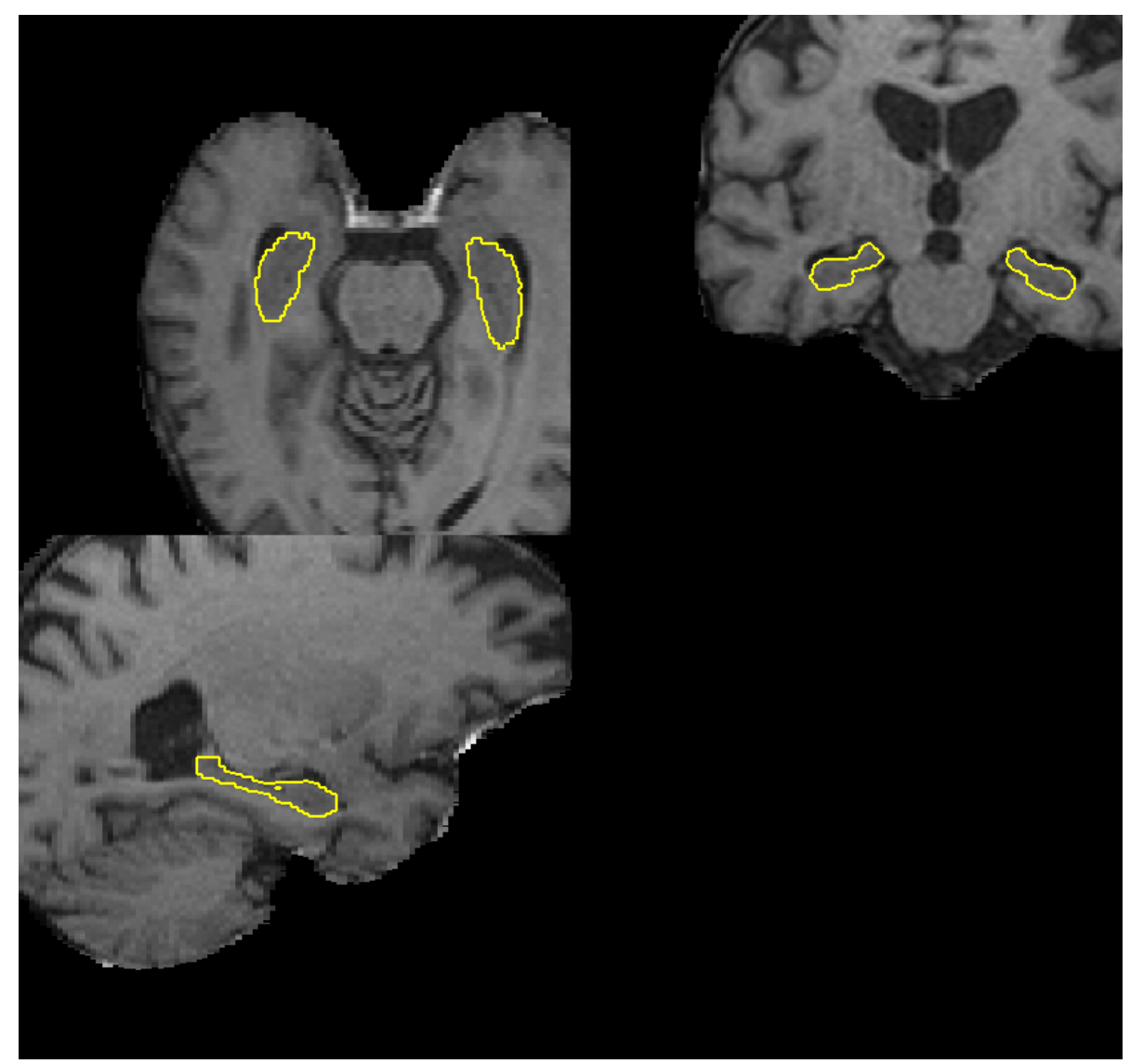


Supplemental Table S2. Added predictive value of a second biomarker for AD-type dementia after 2-year follow-up

\begin{tabular}{llllll}
\hline $\begin{array}{l}\text { Biomarker status } \\
\text { after first test }\end{array}$ & $\begin{array}{l}\text { Second } \\
\text { biomarker tested }\end{array}$ & $\mathrm{N}$ & LR+ & LR- & Odds ratio \\
\hline $\begin{array}{l}\text { CSF A } \beta 1-42 / \mathrm{tau} \\
\text { ratio normal }\end{array}$ & $\mathrm{HCV}$ & 76 & $3.3(1.6-6.8)$ & $0.46(0.19-1.14)$ & $7.1^{*}(1.5-33)$ \\
$\begin{array}{l}\mathrm{CSF} A \beta 1-42 / \mathrm{tau} \\
\text { ratio abnormal }\end{array}$ & $\mathrm{HCV}$ & 77 & $1.5(0.88-2.4)$ & $0.72(0.47-1.11)$ & $2(0.8-5.0)$ \\
$\begin{array}{l}\text { HCV normal } \\
\text { HCV abnormal }\end{array}$ & $\begin{array}{l}\mathrm{CSF} \mathrm{A} \beta 1-42 / \mathrm{tau} \\
\text { ratio }\end{array}$ & 99 & $2.9(2.0-4.3)$ & $0.2(0.07-0.58)$ & $14^{\dagger}(3.9-54)$ \\
& $\begin{array}{l}\mathrm{CSF} \mathrm{A} \beta 1-42 / \mathrm{tau} \\
\text { ratio }\end{array}$ & 54 & $1.6(1.1-2.4)$ & $0.38(0.16-0.93)$ & $4.1^{*}(1.2-14)$ \\
\hline
\end{tabular}

Results are presented with $95 \%$ confidence intervals. $\mathrm{AD}=$ Alzheimer's disease; $\mathrm{MCI}=$ mild cognitive impairment; $\mathrm{CSF}=$ cerebrospinal fluid; HCV=hippocampal volume; $\mathrm{LR}+=$ positive likelihood ratio; LR-=negative likelihood ratio. ${ }^{*} \mathrm{P}<0.05 ;{ }^{\dagger} \mathrm{p}<0.001$.

\section{Supplemental Table S3. Added predictive value of a second biomarker for cognitive decline on MMSE}

\begin{tabular}{|c|c|c|c|c|}
\hline Biomarker status after first test & $\begin{array}{l}\text { Second biomarker } \\
\text { tested }\end{array}$ & $\begin{array}{l}\text { Biomarker } \\
\text { status }\end{array}$ & Slope & $\begin{array}{l}\text { P-value } \\
\text { difference }\end{array}$ \\
\hline \multirow[t]{2}{*}{ CSF $A \beta 1-42 /$ tau ratio normal } & $\mathrm{HCV}$ & Normal & $-0.27 \ddagger(0.1)$ & 0.001 \\
\hline & & Abnormal & $-0.71 \neq(0.1)$ & \\
\hline \multirow[t]{2}{*}{ CSF $\mathrm{A} \beta 1-42 /$ tau ratio abnormal } & $\mathrm{HCV}$ & Normal & $-1.06^{\ddagger}(0.2)$ & 0.333 \\
\hline & & Abnormal & $-1.33 \ddagger(0.2)$ & \\
\hline \multirow[t]{2}{*}{ HCV normal } & CSF $A \beta 1-42 /$ tau ratio & Normal & $-0.19(0.1)$ & $<0.001$ \\
\hline & & Abnormal & $-1.08 \ddagger(0.1)$ & \\
\hline \multirow[t]{2}{*}{ HCV abnormal } & CSF $A \beta 1-42 /$ tau ratio & Normal & $-0.57(0.3)$ & 0.038 \\
\hline & & Abnormal & $-1.35 \ddagger(0.2)$ & \\
\hline
\end{tabular}

Data are slopes (SE) for annual decline on MMSE. A negative slope indicates cognitive decline. As the sample size of the subgroups was different, different slopes may be presented for the same final groups. CSF=Cerebrospinal fluid, HCV=Hippocampal volume, MMSE=Mini Mental State Examination. $¥ \mathrm{P}<0.001$. 


\section{Appendix S1. Neuropsychological tests used to diagnose MCI}

Neuropsychological tests were used to assess cognitive performance in the domains of memory, language, executive function and attention, and visuoconstruction. Because the DESCRIPA study followed the routine practice at each centre, the tests used to assess each domain varied among centers.

At each center, we selected a primary test for each cognitive domain that was the same as or similar to the tests that were used at the other centers (Visser et al 2008). The primary tests to assess memory were the learning and delayed recall measure of the Rey auditory verbal learning test (4 centers) and the word list of the consortium to establish a registry for $\mathrm{AD}$ (CERAD) test battery ( 2 centers). The primary test to assess language was the 1-min verbal fluency for animals (all centers). The primary test to assess executive function and attention was the trail making test (TMT) parts A and B (all centers). The primary tests to assess visuoconstruction were the copy subtest of the Rey-Osterrieth complex figure (4 centers) or the copy of the CERAD figures ( 2 centers). If patients could not be classified on the basis of these primary tests because of missing data, we used alternative tests, qualitative rating by a neuropsychologist, or a subscore on the MMSE.

\section{Appendix S2. Scan parameters and MRI protocols used at each center}

CENTER AMSTERDAM VUMC: Siemens Magnetom Impact Expert 1.0 T, 3D scan, 168 slices, FOV $250 \mathrm{~mm}$, matrix $256 \times 256$; slice thickness $1.5 \mathrm{~mm}$, TE: $7 \mathrm{~ms}$, TR: $15 \mathrm{~ms}$, TI $300 \mathrm{~ms}$, flip angle $15^{\circ}$.

CENTER HUDDINGE: Siemens Avanto 1.5 T, 21 slices, FOV 220 mm, FOV phase 87.1, distance factor 30, phase $\mathrm{R}>\mathrm{L}$, slice thickness $5.0 \mathrm{~mm}$, TE: $96 \mathrm{~ms}$, TR: $4000 \mathrm{~ms}$, flip angle 150。, number of averages 1; and Siemens Symphony 1.5 T, 21 slices, FOV 220 mm, FOV phase 75.0, distance factor 30, phase R>L, slice thickness $5.0 \mathrm{~mm}$, TE: $99 \mathrm{~ms}$, TR: $4100 \mathrm{~ms}$, flip angle $150^{\circ}$, number of averages 2 .

CENTER KUOPIO: Siemens Vision 1.5 T, T1 3D-scan, MPRAGE OBL; COR>TRA, FOV 250, mat $256 \times 256,128$ slices, TR $9.7 \mathrm{~ms}$, TE $4 \mathrm{~ms}$, Slice th $2.0 \mathrm{~mm}$, no slice gap, flip angle 12 。

CENTER MALMO: Siemens Sonata 1.5 T, MPRAGE + lmpr-cor, 144 slices, FOV $250 \mathrm{~mm}$, phase $\mathrm{R}>\mathrm{L}$, TR 1970, TE 3.93, distance factor 50 , slice thickness $1.5 \mathrm{~mm}$, flip angle 15 .

CENTER MUNICH: Siemens Magnetom Vision; 1.5 T; MPRAGE; Slice thickness $1.05 \mathrm{~mm}$, TR 11.4, TE 4.4, TI 300; FOV 256*256; Flip angle 8graden; number of averages 1.

CENTER THESSALONIKI: Siemens Expert Plus unit 1.0 T, 3D-MPR: 15 (TR),7 TE ,8 FLIP ANG., 250 (Slabth), 1,49Ef thick, 168 Partitions, 250 FOV,256x192 Matrix, 1 Aquis. ,ACQ TIME 10,21min. 



\section{CHAPTER 4}

\section{PREDICTION OF ALZHEIMER'S DISEASE IN SUBJECTS WITH AMNESTIC AND NON-AMNESTIC MCI}

Neurology 2013; 80(12):1124-32

Stephanie J.B. Vos, Ineke A. van Rossum, Frans Verhey, Dirk L. Knol, Hilkka Soininen, LarsOlof Wahlund, Harald Hampel, Magda Tsolaki, Lennart Minthon, Giovanni B. Frisoni, Lutz Froelich, Flavio Nobili, Wiesje van der Flier, Kaj Blennow, Robin Wolz, Philip Scheltens, Pieter Jelle Visser 


\section{ABSTRACT}

\section{Objective}

To compare the predictive accuracy of beta amyloid $(A \beta) 1-42$ and total tau in cerebrospinal fluid (CSF), hippocampal volume (HCV), and apolipoprotein E (APOE) genotype for AD-type dementia in subjects with amnestic MCI (aMCI) and non-amnestic MCI (naMCI).

\section{Methods}

We selected 399 subjects with aMCI and 226 subjects with naMCI from a multicenter memory clinic-based cohort. We measured CSF A $\beta 1-42$ and tau by ELISA ( $n=231)$, HCV on MRI (n=388), and APOE- $44(n=523)$. Follow-up was performed annually up to 5 years. Outcome measures were progression to AD-type dementia and cognitive decline.

\section{Results}

At least 1 follow-up was available for 538 subjects (86\%). 132 (38\%) subjects with aMCI and 39 (20\%) subjects with naMCI progressed to AD-type dementia after an average followup of 2.5 years. CSF A $\beta 1-42$, tau and A $\beta 1-42 /$ tau ratio, HCV, and APOE- $\varepsilon 4$ predicted AD-type dementia in each MCI subgroup with the same overall diagnostic accuracy. However, CSF A 1-42 concentration was higher and hippocampal atrophy less severe in subjects with naMCI compared to aMCI. This reduced the sensitivity but increased the specificity of these markers for AD-type dementia in subjects with naMCI.

\section{Conclusions}

$\mathrm{AD}$ biomarkers are useful to predict AD-type dementia in subjects with aMCI and naMCI. Biomarkers may, however, not be as sensitive for early diagnosis of AD in naMCI compared to aMCI. This may have implications for clinical implementation of the NIA-AA criteria. 


\section{INTRODUCTION}

The new research criteria for mild cognitive impairment (MCI) due to Alzheimer's disease (AD) proposed by the National Institute on Aging and Alzheimer's association (NIA-AA) consider both amnestic MCI (aMCI) and non-amnestic MCI (naMCI) as possible prodromal stages of AD-type dementia. ${ }^{1}$ While previous studies on aMCI showed that biomarkers in cerebrospinal fluid (CSF), hippocampal volume (HCV) and apolipoprotein E (APOE) genotype could predict $\mathrm{AD}$-type dementia in subjects with $\mathrm{aMCI},{ }^{2-5}$ the predictive accuracy of these markers in subjects with naMCI remains to be established.

Recent studies showed that AD pathology is common in subjects with naMCI.6 AD may, however, present differently in naMCI than in $\mathrm{aMCI}^{7}$ and $\mathrm{AD}$ biomarkers may therefore have a different predictive accuracy for AD-type dementia. Information on the predictive accuracy of AD biomarkers in naMCI will be important both for clinical practice and AD trial design. ${ }^{8}$

We previously reported the predictive accuracy of HCV and the CSF A $\beta 1-42 /$ tau ratio for AD-type dementia after 2 years in a subset of our subjects with $\mathrm{MCI}^{9}$ and reported the predictive accuracy of neuronal injury markers for cognitive decline in subjects with CSF amyloid pathology ${ }^{10}$. The aim of the present study was to compare the predictive accuracy of biomarkers for AD-type dementia and cognitive decline after up to 5 years in subjects with aMCI with that in subjects with naMCI. As predictors we used CSF beta amyloid (A $\beta) 1$ 42, tau, A $\beta 1-42 /$ tau ratio, HCV, and APOE genotype. We also tested short-term AD prediction after 2 years.

\section{METHODS}

\section{Subjects}

Subjects were recruited from the DESCRIPA cohort ${ }^{11}$ and Alzheimer Center of the VU University medical center (VUmc). ${ }^{12}$ Inclusion criteria were baseline diagnosis of aMCI or naMCI, age $\geq 55$ years, data on either CSF A $\beta 1-42$ and tau, HCV, or APOE genotype, and being newly referred for the assessment of cognitive complaints. Exclusion criteria were diagnosis of dementia at baseline or any other somatic, psychiatric or neurological disorder that might have caused the cognitive impairment.

DESCRIPA is a European multicenter study performed in a memory-clinic setting and enrolled subjects between 2003 and 2005. ${ }^{11}$ For this study, 431 eligible subjects were selected from 16 participating centers in which CSF was collected, MRI was performed, or APOE genotype was determined. The VUmc center was one of the DESCRIPA centers and contributed an additional sample of 194 subjects that were seen outside the DESCRIPA inclusion period with data on CSF, MRI, or APOE measures. No differences in biomarkers were found between subjects from the VUmc center in the DESCRIPA study and those from the additional VUmc sample. Subjects with baseline data on CSF, MRI or APOE genotype $(n=625)$ differed from subjects in whom CSF, MRI, and APOE genotype data were not 
available $(\mathrm{n}=126)$ with regard to gender (female 54 vs. 67\%; $=0.034$ ), and MCI type (aMCI 64 vs. $52 \%$; $=0.003$ ) at baseline.

\section{Standard Protocol Approvals, Registrations, and Patient Consents}

The medical ethics committee at each center approved the study. All subjects provided informed consent.

\section{Clinical and cognitive assessment}

We performed a clinical history, medical and neurological examination, laboratory tests, functional evaluation with the clinical dementia rating scale, rating scales for neuropsychiatric symptoms, scoring on the Mini-Mental State Examination (MMSE), and a neuropsychological assessment.11,12 Data was collected blinded to results of biomarker analyses.

Baseline diagnosis of MCI was made according to the criteria of Petersen et al. ${ }^{13}$ Raw scores on neuropsychological tests were corrected for age, gender, and education, and expressed as z-scores. ${ }^{11}$ Subjects with a z-score $<-1.5$ SD on the learning measure or delayed recall of a word list learning test or equivalent memory test were classified as having aMCI. Subjects with a z-score $<-1.5$ SD on the trail making test (TMT) part A, TMT part B, verbal fluency, Rey figure copy test or equivalent test but no memory impairment were classified as having naMCI. Subjects with aMCI were subclassified as aMCI-single domain (SD) if they only had an impairment in memory and as aMCI-multi domain (MD) if they had also impairments in non-memory domains. We calculated a memory composite score as the average z-score of the learning and delayed recall score of the word list learning test and a cognitive composite score as the average z-score of all 6 cognitive tests, if scores were available for at least 3 tests.

Cerebrovascular measures were hypertension $(n=486$; systolic blood pressure $\geq 140$ mmHG or diastolic blood pressure $\geq 90 \mathrm{mmHG}$ ), hypercholesterol $(\mathrm{n}=483)$, and white matter lesions (WML; $n=361$ ). WML were scored by the PVL Scheltens scale ${ }^{14}$ for the Descripa cohort and by the Fazekas scale ${ }^{15}$ for the additional VUmc sample. We scored the PVL scores as Fazekas score: PVL 0=Fazekas 0, PVL 1-2=Fazekas 1, PVL 3=Fazekas 2, and PVL $\geq 4$ $=$ Fazekas 3. Final WML scores were dichotomized as 0-1 vs. 2-3.

Follow-up assessment was performed annually up to 5 years. Primary outcome measures were conversion to AD-type dementia at follow-up according to the DSM-IV ${ }^{16}$ and NINCDS-ADRDA criteria ${ }^{17}$ and annual cognitive decline on MMSE, memory and overall cognition. Secondary outcome measure was short-term conversion to AD-type dementia after 2 years.

\section{CSF analyses}

CSF data were available for 231 subjects. These subjects showed minor differences in age (69.7 vs. 71.3; $\mathrm{p}=0.035$ ), MMSE (26.6 vs. 27.1;p=0.042), and gender (female 44 vs. 
$59 \% ; p=0.02$ ) compared to subjects without CSF data. 214 subjects with CSF data had a least one follow-up assessment.

CSF was collected by lumbar puncture, centrifuged, and stored at $-80^{\circ} \mathrm{C}$ in polypropylene tubes. Three samples were thawed twice but analyses without these samples revealed similar results. CSF A $\beta 1-42$ and total tau ( $\mathrm{t}$-tau) were measured by experienced technicians using commercially available sandwich ELISAs (Innotest $\beta$-amyloid 1-42; Innotest hTAU-Ag; Innogenetics, Ghent, Belgium), specially constructed to measure A $\beta 1-42$ and $\mathrm{t}$-tau, ${ }^{18,19}$ at the lab in Gothenburg for the DESCRIPA cohort and in Amsterdam for the additional subjects of the VUmc cohort. We corrected for interlaboratory ELISA differences by analyzing 33 samples at both labs and we adjusted VUmc values to those of DESCRIPA using the following formula: Gothenborg=(SD Gothenborg/SD VUmc)*VUmc+average Gothenborg-((SD Gothenborg /SD VUmc)*average VUmc). ${ }^{20}$

The CSF measures were dichotomized based on prespecified cut-offs. ${ }^{21,22}$ The cut-off was $<500 \mathrm{pg} / \mathrm{ml}$ for $\mathrm{A} \beta 1-42,>450 \mathrm{pg} / \mathrm{ml}$ for t-tau in subjects below 70 years and $>500$ $\mathrm{pg} / \mathrm{ml}$ for t-tau in subjects above 70 years, and $A \beta 1-42 /(240+[1.18 * t-t a u])<1.0$ for $A \beta 1$ $42 / \mathrm{t}$-tau ratio.

\section{MRI analyses}

MRI scans that allowed automated measurements of HCV were available for 388 subjects. These subjects did not differ from subjects without data of automatically measured HCVs on demographical variables. 329 subjects with MRI data had a least one follow-up assessment.

For the DESCRIPA cohort, at each site, subjects were scanned according to the routine MRI protocol. All scanning was performed at 1.0 or $1.5 \mathrm{~T}$ and included a three-dimensional T1-weighted gradient echo sequence and a fast fluid attenuated inversion recovery (FLAIR) sequence. ${ }^{9} \mathrm{HCV}$ was measured centrally at the Department of Computing at Imperial College London, using learning embeddings for atlas propagation (LEAP), an automated segmentation technique based on atlas registration., 23

The sum of the left and right HCV was dichotomized by defining a cut-off $\left(5.389 \mathrm{~cm}^{3}\right)$ that could best differentiate healthy controls from subjects with AD-type dementia in the ADNI cohort, based on the Youden index from a time-dependent ROC curve in R. ${ }^{24}$ The cutoff was similar to the optimal cut-off in our own cohort. ${ }^{9}$

\section{APOE genotype}

APOE data were available for 523 subjects. These subjects did not differ from subjects without APOE data on demographical variables. 464 subjects with APOE data had a least one follow-up assessment. APOE genotype was determined by polymerase chain reaction of genomic DNA extracted from EDTA anticoagulated blood. Subjects were classified as APOEe4 carriers or non-carriers. 


\section{Statistical analyses}

Statistical analyses were done with SPSS version 19.0 (Chicago, IL, USA) and statistical software package $\mathrm{R}$ version $2.10 .1,^{25}$ with significance set at $\mathrm{p}<0.05$. All analyses were corrected for age, gender, and education.

We calculated hazard ratios (HRs) using Cox regression to investigate the predictive accuracy for AD-type dementia of CSF A $\beta 1-42$, tau, A $\beta 1-42 /$ tau ratio, $\mathrm{HCV}$, and APOE genotype in both MCI groups. For analyses with continuous variables, variables were log transformed and expressed as z-scores.

The relation between biomarkers and change on the MMSE, memory, and overall cognition in each MCI subgroup was assessed by slope analyses with mixed models. The analyses included the baseline score and available follow-up scores up to 5 years. The model was specified with center as a random effect, as this model provided the best $-2 \log$ likelihood compared to models with simpler covariance structures. The median number of measurements per subject was 3 (range 1-6) for the MMSE and 2 (range 1-6) for memory with minor differences between MCI subgroups and between predictor variables.

We calculated odds ratios (ORs) to investigate predictive accuracy for AD-type dementia after 2 years. Additionally, we calculated sensitivity, specificity, positive predictive value, and negative predictive value.

\section{RESULTS}

\section{Sample characteristics}

$399(64 \%)$ subjects were classified as aMCI and 226 (36\%) as naMCI. Of the aMCI subjects, 174 (44\%) had aMCI-SD and 225 (56\%) aMCI-MD. Subjects with aMCI had poorer MMSE scores and memory scores, better TMT part A scores, lower levels of CSF A $\beta 1-42$, higher levels of CSF tau, a lower CSF A $\beta 1-42$ /tau ratio, and a smaller HCV than subjects with naMCI at baseline (table 1). Subjects with aMCI-MD had poorer cognitive scores than subjects with aMCI-SD but they did not differ in biomarker scores. 538 (86\%) subjects had at least one follow-up assessment (average 2.5 years $(S D=1.0)$ ). Progression rate to AD-type dementia was higher in aMCI (38\%) than in naMCI (20\%), and higher in aMCI-MD (46\%) than in aMCI-SD (29\%). 17 (5\%) of the subjects with aMCI progressed to other dementias (7 frontotemporal dementia, 7 Lewy body dementia, 1 Parkinson dementia, 1 alcohol dementia, and 1 vascular dementia) and $15(8 \%)$ of the subjects with naMCI progressed to other dementias ( 3 frontotemporal dementia, 6 Lewy body dementia, 5 vascular dementia, 1 normal pressure hydrocephalus). These subjects were included in the no-AD dementia group. 
Table 1. Baseline characteristics of subjects with aMCI and naMCI

\begin{tabular}{|c|c|c|c|c|}
\hline & $\begin{array}{l}\text { naMCI } \\
(n=226)\end{array}$ & $\begin{array}{l}\text { aMCI } \\
(n=399)\end{array}$ & $\begin{array}{l}\text { aMCI single } \\
\text { domain } \\
(\mathrm{n}=174)\end{array}$ & $\begin{array}{l}\text { aMCI multi } \\
\text { domain } \\
(\mathrm{n}=225)\end{array}$ \\
\hline Age & $70.7(7.6)$ & 70.7 (7.8) & $70.3(7.6)$ & $71.0(8.0)$ \\
\hline Female, $\mathrm{n}$ & $111(49 \%)$ & $224(56 \%)$ & $76(44 \%)^{\ddagger}$ & $148(66 \%)^{* *}$ \\
\hline Years of education & $10.0(4.1)$ & $10.2(3.9)$ & $10.8(3.5)^{\pi}$ & $9.8(4.1)$ \\
\hline CDR-SOB & $1.1(1.0)$ & $1.3(1.0)$ & $1.2(1.0)$ & $1.4(1.0)^{*}$ \\
\hline MMSE & $27.5(2.1)$ & $26.5(2.5)^{* * *}$ & $27.1(2.2)^{\ddagger}$ & $26.1(2.6)^{* * *}$ \\
\hline Memory score (z-score) & $-0.3(0.7)$ & $-2.0(0.6)^{* * *}$ & $-1.8(0.5)^{* * *} \neq$ & $-2.1(0.6)^{* * *}$ \\
\hline Verbal fluency (z-score) & $-0.6(1.2)$ & $-0.8(1.2)$ & $-0.3(1.0)^{* *} \ddagger$ & $-1.2(1.2)^{* * *}$ \\
\hline TMT part A (z-score) & $-1.2(1.7)$ & $-0.8(1.6)^{*}$ & $0.1(0.8)^{* * *} \ddagger$ & $-1.5(1.8)$ \\
\hline TMT part B (z-score) & $-1.5(1.9)$ & $-1.2(1.9)$ & $0.04(0.8)^{* * *} \neq$ & $-2.2(1.9)^{* * *}$ \\
\hline Visuoconstruction (z-score) & $-0.1(1.3)$ & $0.1(1.1)$ & $0.6(0.7)^{* * * \neq}$ & $-0.2(1.2)$ \\
\hline $\mathrm{A} \beta 1-42, \mathrm{pg} / \mathrm{ml}$ & $624(283)$ & $550(267)^{* *}$ & $589(270)$ & $512(261)^{*}$ \\
\hline Tau, pg/ml & 427 (261) & $524(322)^{*}$ & $533(300)^{*}$ & $488(241)$ \\
\hline Ratio A $\beta 1-42$ /tau & $0.96(0.6)$ & $0.78(0.5)^{*}$ & $0.84(0.5)$ & $0.73(0.5)^{*}$ \\
\hline Hippocampal volume, $\mathrm{cm}^{3}$ & $5.89(0.7)$ & $5.55(0.8)^{* * *}$ & $5.59(0.8)^{* *}$ & $5.53(0.8)^{* * *}$ \\
\hline APOE- $\varepsilon 4, n$ & $73(40 \%)$ & $158(47 \%)$ & $79(54 \%)^{* \pi}$ & $79(41 \%)$ \\
\hline Hypertension, $\mathrm{n}$ & $97(47 \%)$ & $144(41 \%)$ & $50(34 \%)^{* \pi}$ & $94(46 \%)$ \\
\hline Hypercholesterol, n & $56(27 \%)$ & $116(33 \%)$ & $39(27 \%)^{\pi}$ & $77(38 \%)^{*}$ \\
\hline White matter hyperintensities, $\mathrm{n}$ & $60(37 \%)$ & $79(30 \%)$ & $37(29 \%)$ & $42(31 \%)$ \\
\hline Follow-up, n & $192(85 \%)$ & $346(87 \%)$ & $155(89 \%)$ & $191(85 \%)$ \\
\hline AD conversion rate, $\mathrm{n}$ & $39(20 \%)$ & $132(38 \%)^{* * *}$ & $45(29 \%)^{\dagger}$ & $87(46 \%)^{* * *}$ \\
\hline No-AD dementia conversion rate, $\mathrm{n}$ & $15(8 \%)$ & $17(5 \%)$ & $3(2 \%)$ & $14(7 \%)$ \\
\hline Time to $\mathrm{AD}, \mathrm{y}$ & $1.9(1.1)$ & $1.9(1.0)$ & $2.2(1.2)$ & $1.8(1.0)$ \\
\hline $\begin{array}{l}\text { Follow-up time non-converters and } \\
\text { no-AD dementia converters, y }\end{array}$ & $2.6(1.0)$ & $2.4(1.0)$ & $2.4(1.0)$ & $2.3(1.0)$ \\
\hline
\end{tabular}

Data are mean (SD), unless specified otherwise. Sample size APOE: aMCI n=339, naMCI n=184; hippocampal volume: aMCI $n=241$, naMCI $n=147$; CSF: aMCI n=156, naMCI $n=75$; blood pressure: aMCI $n=353$, naMCI $n=205$; cholesterol: aMCI $n=351$, naMCI $n=204$; white matter lesions: aMCI $n=260$, naMCI $n=164$. aMCI=amnestic mild cognitive impairment, naMCI=non-amnestic mild cognitive impairment, single=single domain impaired (memory), multi=multiple domains impaired, CDR-SOB=Clinical Dementia Rating Scale Sum of Boxes, MMSE=Mini-Mental State Examination, $\mathrm{TMT}=$ trail making test, $\mathrm{A} \beta 1-42=$ beta amyloid 1-42, APOE=apolipoprotein $\mathrm{E}, \mathrm{AD}=\mathrm{Alzheimer}$ 's disease. ${ }^{*} \mathrm{P}<0.05,{ }^{* *} \mathrm{p}<0.01,{ }^{* * *} \mathrm{p}<0.001$ for aMCI all, single aMCI or multi aMCI compared to naMCI; $\uparrow \mathrm{p}<0.05,+\mathrm{p}<0.01,{ }^{\ddagger} \mathrm{p}<0.001$ for aMCI single domain compared to aMCI multi domain. 
Table 2. Predictive accuracy of biomarkers for AD-type dementia

\begin{tabular}{|c|c|c|c|c|c|}
\hline Biomarkers & Group & $\begin{array}{l}\text { HR for continuous } \\
\text { predictor variable }\end{array}$ & P-value & $\begin{array}{l}\text { HR for } \\
\text { dichotomous } \\
\text { predictor variable }\end{array}$ & P-value \\
\hline \multirow[t]{4}{*}{ CSF A $\beta 1-42$} & naMCI & $1.8(1.0-3.1)$ & 0.034 & $3.3(1.4-7.9)$ & 0.008 \\
\hline & aMCI & $1.7(1.3-2.2)$ & $<0.001$ & $3.5(2.0-6.3)$ & $<0.001$ \\
\hline & aMCI single & $2.0(1.4-3.0)$ & $<0.001$ & $3.8(1.6-9.0)$ & 0.002 \\
\hline & aMCI multi & $1.7(1.1-2.4)$ & 0.007 & $3.2(1.4-7.4)$ & 0.005 \\
\hline \multirow[t]{4}{*}{ CSF t-tau } & naMCI & $2.7(1.6-4.6)$ & $<0.001$ & $5.2(2.1-13)$ & $<0.001$ \\
\hline & aMCI & $2.1(1.5-2.9)$ & $<0.001$ & $3.0(1.7-5.3)$ & $<0.001$ \\
\hline & aMCI single & $2.6(1.5-4.5)$ & 0.001 & $3.9(1.6-9.9)$ & 0.004 \\
\hline & aMCI multi & $2.0(1.2-3.4)$ & 0.006 & $2.2(1.0-7.4)$ & 0.034 \\
\hline \multirow[t]{4}{*}{ CSF ratio } & naMCI & $2.6(1.6-4.4)$ & $<0.001$ & $7.1(1.6-31)$ & 0.009 \\
\hline & aMCI & $2.0(1.6-2.6)$ & $<0.001$ & $14(3.4-58)$ & $<0.001$ \\
\hline & aMCI single & $2.1(1.5-3.0)$ & $<0.001$ & $17(2.3-127)$ & 0.006 \\
\hline & aMCI multi & $2.5(1.6-3.8)$ & $<0.001$ & $12(1.5-87)$ & 0.018 \\
\hline \multirow[t]{4}{*}{$\mathrm{HCV}$} & naMCI & $2.1(1.1-3.8)$ & 0.016 & $2.3(0.9-6.0)$ & 0.085 \\
\hline & $\mathrm{aMCI}$ & $1.6(1.3-2.1)$ & $<0.001$ & $3.0(1.8-4.9)$ & $<0001$ \\
\hline & aMCI single & $1.4(0.9-2.1)$ & 0.107 & $2.2(1.0-4.8)$ & 0.045 \\
\hline & aMCI multi & $1.8(1.3-2.5)$ & $<0.001$ & $3.1(1.6-6.0)$ & 0.001 \\
\hline \multirow[t]{4}{*}{ APOE-e4 } & naMCI & - & & $1.9(0.9-3.9)$ & 0.085 \\
\hline & aMCI & - & & $1.8(1.2-2.6)$ & 0.003 \\
\hline & aMCI single & - & & $5.6(2.2-14)$ & $<0.001$ \\
\hline & aMCI multi & - & & $1.3(0.8-2.1)$ & 0.215 \\
\hline
\end{tabular}

Data are hazard ratios $(95 \% \mathrm{CI})$, corrected for age, gender, and education. Scores were dichotomized based on prespecified cut-offs (see methods). $\mathrm{AD}=$ Alzheimer's disease, $\mathrm{aMCI}=$ amnestic mild cognitive impairment, naMCI=nonamnestic mild cognitive impairment, $\mathrm{CSF}=$ cerebrospinal fluid, A $31-42=$ beta amyloid 1-42, HCV=hippocampal volume, APOE=apolipoprotein E. Hazard ratio aMCI vs naMCI: all p>0.12.

\section{Prediction of AD-type dementia}

CSF A $\beta 1-42$, tau, A $\beta 1-42 /$ tau ratio, and HCV all predicted AD-type dementia during the 5year follow-up both in subjects with aMCI and naMCI (table 2). When scores were dichotomized, similar results were obtained. Also presence of the APOE- $\varepsilon 4$ allele predicted AD-type dementia in each MCI group, but with only a trend in naMCI $(\mathrm{p}=0.085)$. The HRs for AD-type dementia did not differ between aMCI and naMCI (all $\mathrm{p}>0.12$ ). They did also not differ between aMCI-SD and aMCI-MD, except for APOE- $\varepsilon 4$. However, the HR of APOE-e4 was not different between naMCI and aMCI subtypes.

The ability of the biomarkers to predict outcomes after 2 years was generally similar to what was seen at the longer time point for aMCI vs. naMCI (table 3) and for aMCI-SD vs. aMCI-MD (data not shown). However, CSF and MRI markers showed a higher sensitivity and lower specificity in aMCI than naMCI (table 3 ) because both aMCI subjects with and without AD-type dementia at follow-up had more abnormal biomarker scores compared to subjects with naMCI (supplemental table e-1). 


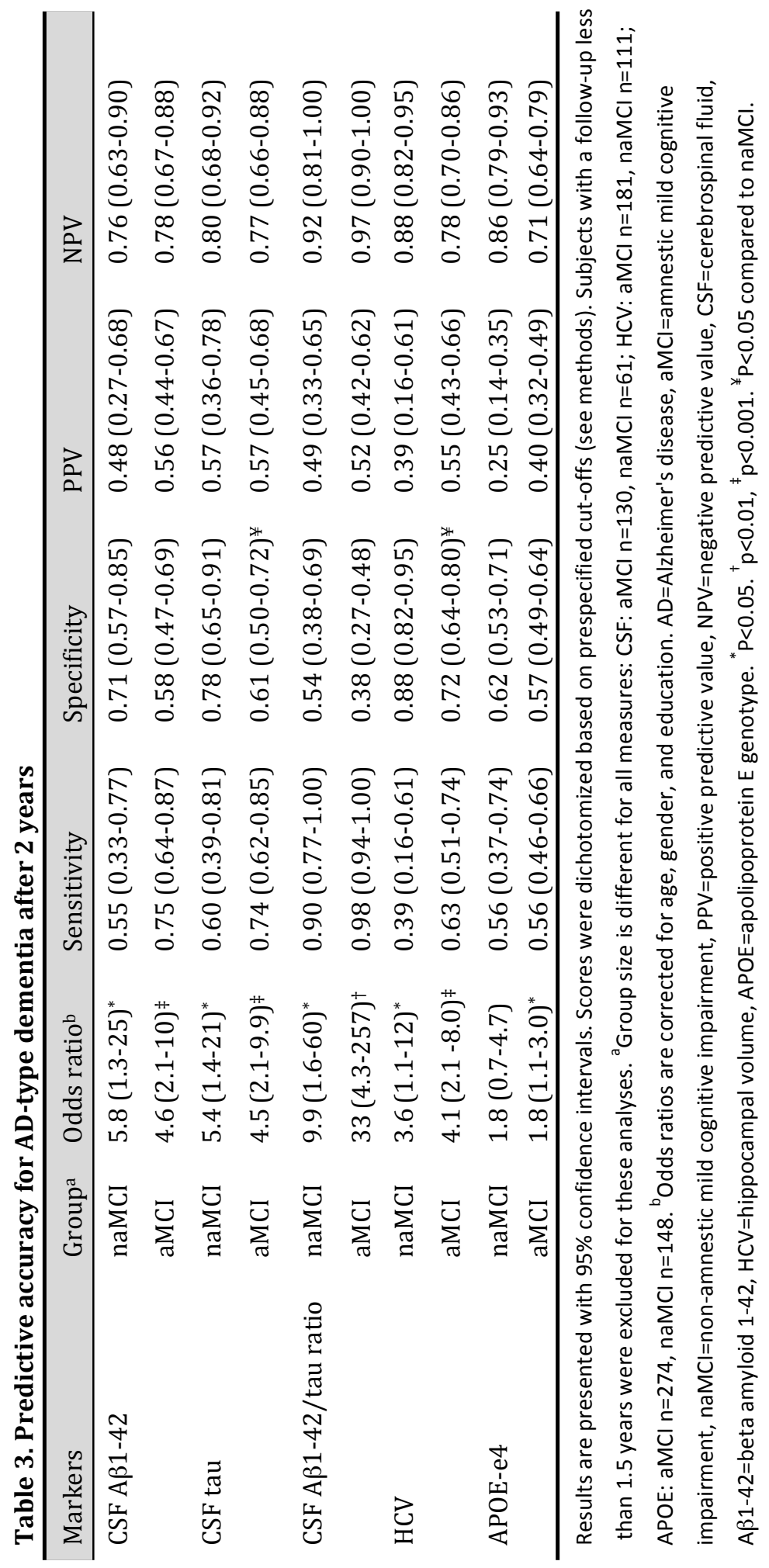




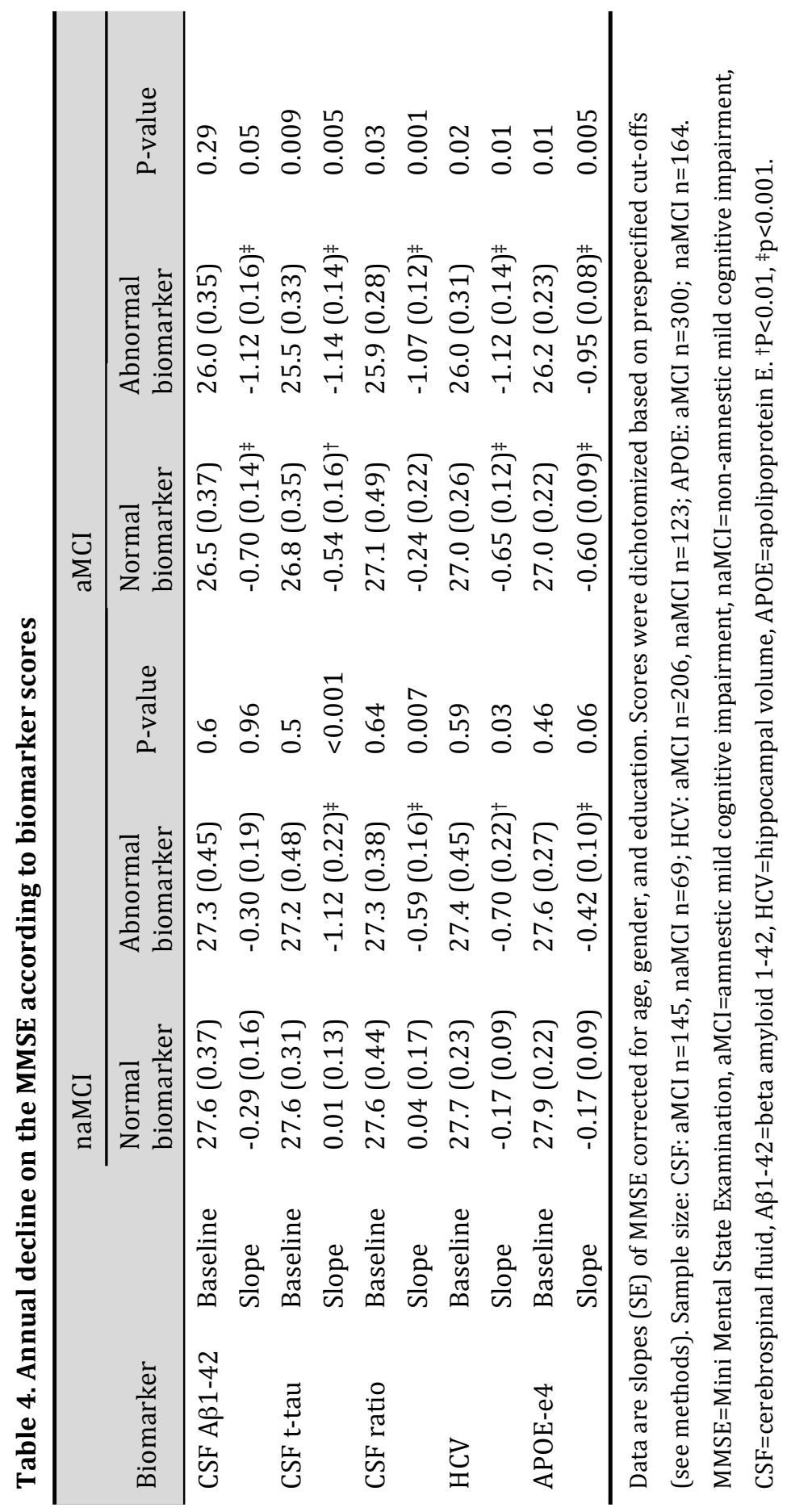


MMSE
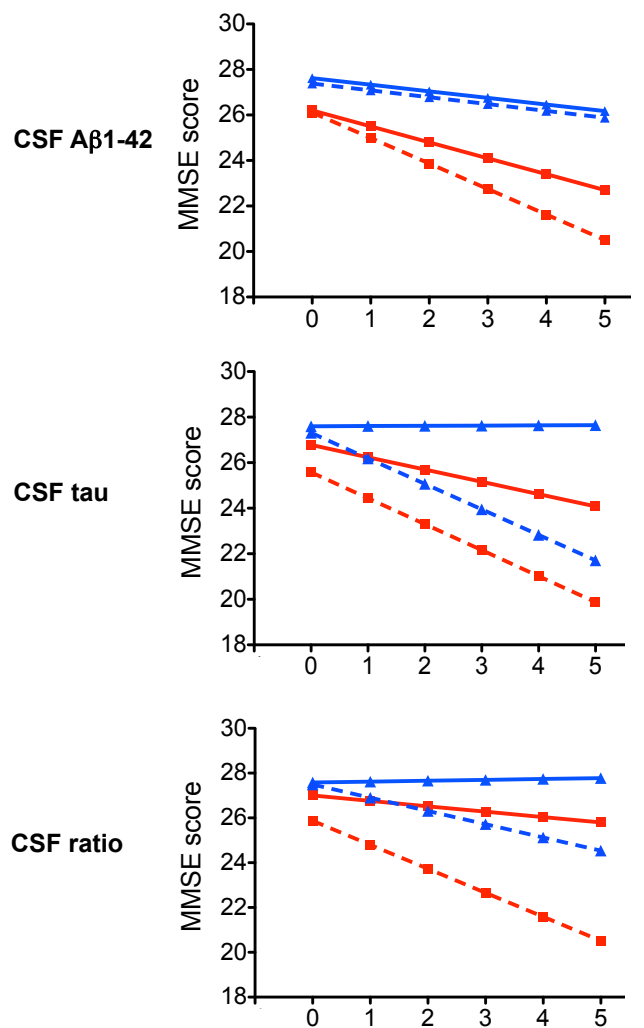

HCV
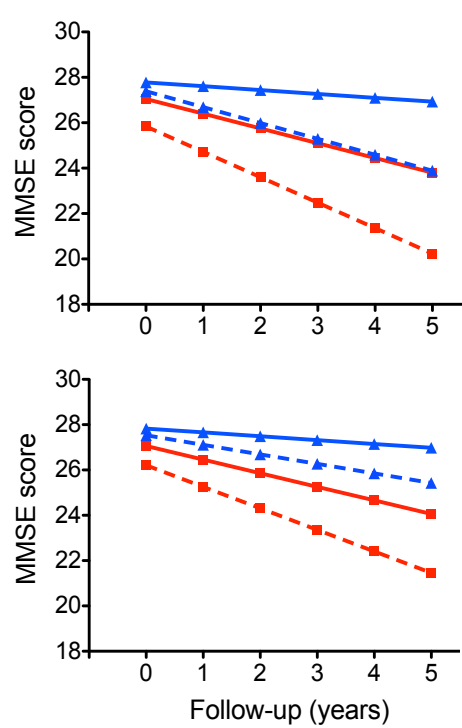

Memory
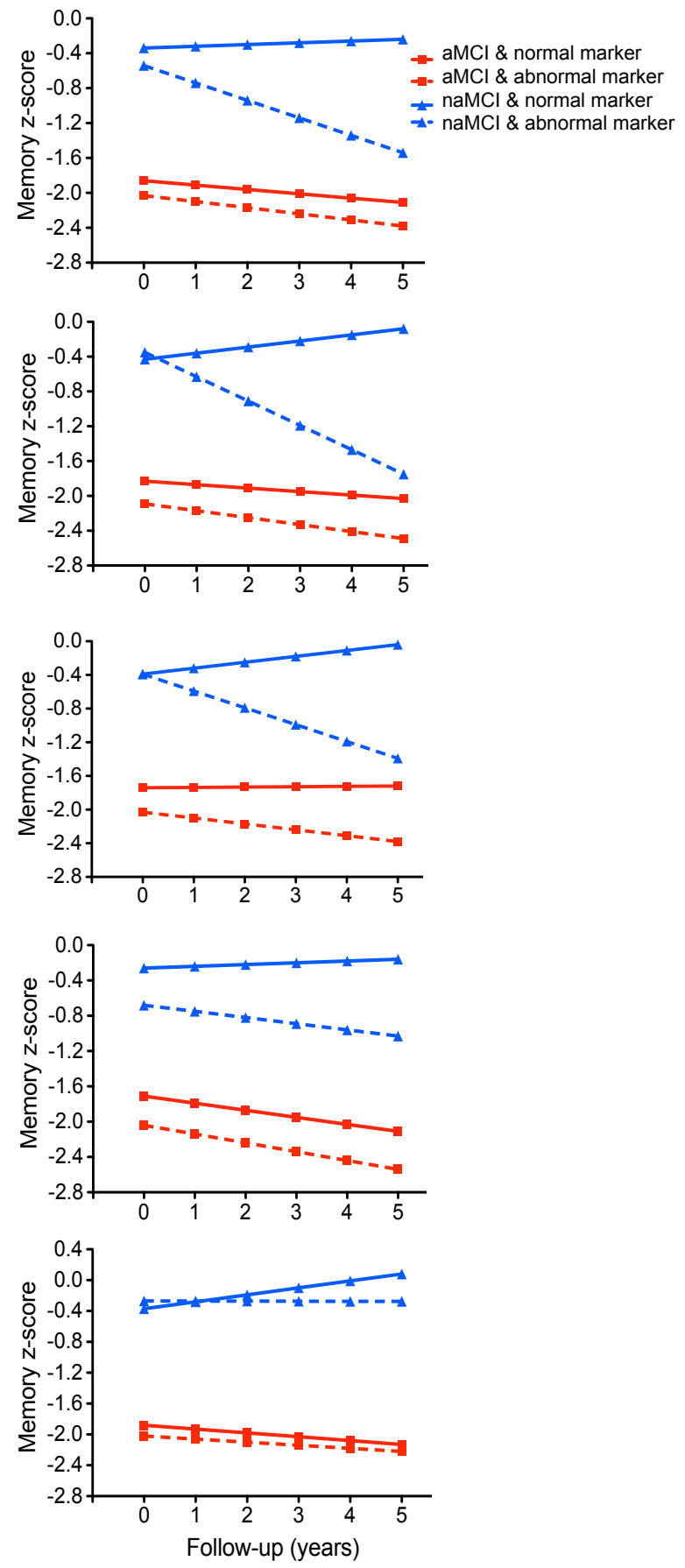

Figure 1. Annual decline on MMSE and memory scores in subjects with aMCI and naMCI by biomarker scores.

The figure shows slopes of MMSE scores (left) and memory z-scores (right) for subjects with aMCI and naMCI according to baseline biomarker profiles of CSF A $\beta 1-42$, CSF tau, CSF A $1-42 /$ tau ratio, HCV, and APOE genotype. Red solid lines (squares) represent subjects with aMCI and a normal biomarker score; Red dotted lines (squares) represent subjects with aMCI and an abnormal biomarker score; Blue solid lines (triangles) represent subjects with naMCI and a normal biomarker score; Blue dotted lines (triangles) represent subjects with naMCI and an abnormal biomarker score. aMCI=amnestic mild cognitive impairment; naMCI=non-amnestic mild cognitive impairment; MMSE=Mini-Mental State Examination; A $\beta 1-42=$ beta amyloid 1-42; HCV=hippocampal volume; APOE=apolipoprotein E. 


\section{Prediction of annual cognitive decline}

All biomarkers predicted decline on the MMSE in subjects with aMCI and naMCI, except for CSF A $\beta 1-42$ in naMCI (table 4, figure 1). In subjects with aMCI, none of the biomarkers predicted change in memory score or overall cognition (figure 1 , supplemental table e-2). In subjects with naMCI, CSF $A \beta 1-42$, tau, and $A \beta 1-42 /$ tau ratio predicted decline in memory and overall cognition, with only a trend for CSF A $\beta 1-42$ (figure 1, supplemental table e-2). Results remained similar after exclusion of subjects who progressed to no-AD dementias. Slopes did not differ between aMCI-SD and aMCI-MD, except for APOE- $\varepsilon 4$ and decline on the MMSE and the CSF ratio and decline in memory (data not shown).

\section{Post-hoc analyses}

Since CSF and MRI biomarker scores differed between subjects with aMCI and naMCI, we calculated subgroup specific cut-offs. In each MCI group, we defined a cut-off point that maximized the Youden index for the prediction of AD-type dementia after 2 years. For subjects with aMCI, the cut-offs were similar to the prespecified cut-offs, except for the CSF A $\beta 1-42 /$ tau ratio for which a lower cut-off was selected. For subjects with naMCI, the optimal cut-offs for CSF A $\beta 1-42$ and HCV were substantially different from the prespecified cut-offs (supplemental table e-3). Using these cut-off points, the odds ratio remained similar to the predictive accuracy using the prespecified cut-offs. The sensitivity increased but at the cost of a decrease in specificity (supplemental table e-3).

\section{DISCUSSION}

This study tested the predictive accuracy of CSF markers, MRI markers and genotype for AD-type dementia in subjects with naMCI. We found that the predictive accuracy was similar to that in subjects with aMCI. Biomarkers also predicted decline on the MMSE in both MCI groups and decline in memory and overall cognition in subjects with naMCI. While there are differences in the relationship of biomarkers to outcome in aMCI and naMCI, our findings suggest that the symptomatic manifestations of the $A D$ pathophysiological process is broader than the amnestic presentation at the MCI stage and also includes non-amnestic MCI.

Although the overall predictive accuracy for AD-type dementia was similar for subjects with aMCI and naMCI, sensitivity of CSF and MRI markers was higher and specificity lower in subjects with aMCI. While most of the differences in sensitivity and specificity between MCI subtypes were not statistically significant, they may be clinically relevant when biomarker cut-offs are applied to define abnormal AD markers and predict clinical progression. Differences were observed because subjects with aMCI had more abnormal biomarker scores than subjects with naMCI. The finding that subjects with naMCI and AD-type dementia at follow-up had less abnormal amyloid markers than subjects with aMCI and AD-type dementia is remarkable. This suggests that subjects with naMCI may have comorbidity, for example vascular lesions, such that less Alzheimer pathology is needed to 
reach the dementia threshold. ${ }^{26}$ However, post-hoc analyses showed no difference in white matter hyperintensities, blood pressure, and cholesterol levels between subjects with naMCI and aMCI with AD-dementia at follow-up (supplemental table e-2). Alternatively, subjects with naMCI may be in an earlier stage of the disease. This would be supported by higher MMSE and cognitive scores, and larger HCV in subjects with naMCI at baseline. However, the time to dementia was the same in subjects with naMCI and aMCI. It is also possible that naMCI is an atypical presentation of AD with a different expression of amyloid pathology in CSF.

The biomarkers predicted decline on MMSE scores generally equally well in subjects with aMCI and naMCI. However, biomarkers predicted decline in memory and overall cognition only in subjects with naMCI but not in subjects with aMCI. This was probably because of floor effects on the memory tests in subjects with aMCI, which reduced the ability to detect further cognitive decline.

For dichotomized measures, CSF A $\beta 1-42 /$ tau ratio was the best predictor for AD-type dementia in each MCI subgroup. This is consistent with recent meta-analyses that showed that the combination of these markers is a better predictor than each separate CSF marker and that the odds ratio of APOE genotype for AD-type dementia is lower than that of CSF and MRI measures. ${ }^{27,28}$ The odds ratio for AD-type dementia of the CSF ratio in subjects with aMCI was very high $(>30)$. However, because only one subject with AD at follow-up had a normal ratio, the $95 \%$ confidence interval was very wide, which limits the interpretation of this finding.

The observation that APOE- $\varepsilon 4$ frequency was similar between subjects with aMCI and naMCI is inconsistent with previous studies showing that the absence of APOE- 44 is related to less memory impairment. ${ }^{29} \mathrm{It}$ is possible that subjects with naMCI in our study have had a late onset of memory impairment as we found that subjects with naMCI and abnormal AD biomarkers showed a rapid decline in memory at follow-up.

Progression rate to AD-type dementia was higher in aMCI than in naMCI while progression to no-AD dementia was similar for MCI subtypes, which is in line with a previous study. ${ }^{30}$ This made also the ratio of $\mathrm{AD}$ dementia converters to no-AD dementia converters different between the groups ( $p=0.005)$.

Our study had several limitations. As the findings were based on memory clinic populations, they may not be generalized to other settings. Biomarkers were analyzed in different subgroups which optimized statistical power for each analysis but which limited the opportunity to compare the predictive accuracy between biomarkers since differences in accuracy could result form differences in subject characteristics. For the main objective of our study, however, this was not a limitation as we aimed to compare the predictive accuracy between MCI subtypes for each biomarker. Moreover, post-hoc analysis in subjects with all biomarkers available (aMCI $n=91$, naMCI $n=42$ ) showed that biomarkers had a generally similar hazard ratio for AD-type dementia compared to the analysis in the total sample. There was variability in scanners used, which may have influenced the volumetric measurements and predictive accuracy of the HCV. Nevertheless, the LEAP approach has 
been shown to be robust for scanner variability. ${ }^{20}$ In addition, the diagnosis of AD-type dementia at follow-up was not neuropathologically validated. This may have led to misclassification. The major strengths of this study were the large sample size and relatively long follow-up period. However, not all subjects had a 5-year follow-up. Furthermore, the multicenter design favors generalization to other memory clinic settings.

Our findings suggest that AD biomarkers are useful to predict AD-type dementia both in subjects with aMCI and naMCI. The differences in sensitivity and specificity for AD-type dementia may have important implications for clinical implementation of the NIA-AA criteria and AD trial design. AD biomarkers, and especially CSF A $\beta 1-42$ levels, may not be as sensitive for early diagnosis of AD in subjects with naMCI compared to aMCI when the same cut-offs are applied in each subgroup. More lenient cut-offs may optimize the sensitivity in subjects with naMCI, however, at the expense of a decrease in specificity. Longitudinal biomarker studies are needed to investigate the change in biomarkers over time to clarify the pathology and prognosis of subjects with naMCI. 


\section{REFERENCES}

1. Albert MS, DeKosky ST, Dickson D, et al. The diagnosis of mild cognitive impairment due to Alzheimer's disease: Recommendations from the National Institute on Aging and Alzheimer's Association workgroup. Alzheimers Dement 2011;7:270-279.

2. Landau SM, Harvey D, Madison CM, et al. Comparing predictors of conversion and decline in mild cognitive impairment. Neurology 2010;75:230-238.

3. Shaw LM, Vanderstichele H, Knapik-Czajka M, et al. Cerebrospinal fluid biomarker signature in Alzheimer's Disease Neuroimaging Initiative subjects. Ann Neurol 2009;65(4), 403-413.

4. Devanand DP, Pradhaban G, Liu X, et al. Hippocampal and entorhinal atrophy in mild cognitive impairment: Prediction of Alzheimer disease. Neurology 2007; 68:828-836.

5. Fleisher AS, Sowell BB, Taylor C, et al. Clinical predictors of progression to Alzheimer disease in amnestic mild cognitive impairment. Neurology 2007;68:1588-1595.

6. Schneider JA, Arvanitakis Z, Leurgans SE, Bennett DA. The neuropathology of probable Alzheimer disease and mild cognitive impairment. Ann Neurol 2009;66:200-208.

7. Visser PJ, Verhey F, Knol DL, et al. Prevalence and prognostic value of CSF markers of Alzheimer's disease pathology in patients with subjective cognitive impairment or mild cognitive impairment in the DESCRIPA study: a prospective cohort study. Lancet Neurol 2009;8:619-627.

8. Hampel H, Wilcock G, Andrieu S, et al. Biomarkers for Alzheimer's disease therapeutic trials. Prog Neurobiol 2011;95(4):579-593.

9. Vos S, van Rossum I, Burns Leah, et al. Test sequence of CSF and MRI biomarkers for prediction of AD in subjects with MCI. Neurobiol Aging 2012;33:2272-2281.

10. van Rossum IA, Vos SJB, Burns L, et al. Injury markers predict time to dementia in subjects with MCI and amyloid pathology. Neurology 2012;79(17):1809-1816.

11. Visser PJ, Verhey FRJ, Boada M, et al. Development of Screening Guidelines and Clinical Criteria for Predementia Alzheimer's Disease. Neuroepidemiology 2008;30:254-265.

12. Bouwman FH, Schoonenboom SNM, van der Flier WM, et al. CSF biomarkers and medial temporal lobe atrophy predict dementia in mild cognitive impairment. Neurobiol Aging 2007;28:1070-1074.

13. Petersen RC. Mild cognitive impairment as a diagnostic entity. J Intern Med 2004; 256(3):183-194.

14. Scheltens $\mathrm{P}, \mathrm{Barkhof} \mathrm{F}$, Leys $\mathrm{D}$, et al. A semiquantative rating scale for the assessment of signal hyperintensities on magnetic resonance imaging. J Neurologic Sci 1993;114: 7-12.

15. Fazekas F, Kleinert R, Offenbacher $\mathrm{H}$, et al. Pathologic correlates of incidental MRI white matter signal hyperintensities. Neurology. 1993;43:1683-1689.

16. American Psychiatric Association: Diagnostic and Statistical Manual of Mental Disorders, 4th ed. Washington DC: American Psychiatric Association, 1994.

17. McKhann G, Drachman D, Folstein M, Katzman R, Price D, EM S. Clinical diagnosis of Alzheimer's disease: report of the NINCDS-ADRDA Work-Group under the auspices of the Department of Health and Human Services Task Force on Alzheimer's disease. Neurology 1984;34:939-944.

18. Andreasen N, Hesse C, Davidsson P, et al. Cerebrospinal Fluid \{beta\}-Amyloid(1-42) in Alzheimer Disease: Differences Between Early- and Late-Onset Alzheimer Disease and Stability During the Course of Disease. Arch Neurol 1999;56:673-680.

19. Blennow K, Wallin A, Ågren H, Spenger C, Siegfried J, Vanmechelen E. Tau protein in erebrospinal fluid: a biochemical diagnostic marker for axonal degeneration in Alzheimer's disease? Mol Chem Neuropathol 1995;26:231-245.

20. Kolen M, Brennan R. Test Equating: Methods and Practices. New York: Springer-Verlag, 1995. 
21. Sjogren M, Vanderstichele H, Ågren H, et al. Tau and Aß42 in Cerebrospinal Fluid from Healthy Adults 21-93 Years of Age: Establishment of Reference Values. Clinical Chemistry 2001;47:17761781.

22. Hulstaert F, Blennow K, Ivanoiu A, et al. Improved discrimination of AD patients using [beta]amyloid(1-42) and tau levels in CSF. Neurology 1999;52:1555-1562.

23. Wolz R, Aljabar P, Hajnal JV, Hammers A, Rueckert D. LEAP: Learning embeddings for atlas propagation. NeuroImage 2010;49:1316-1325.

24. Heagerty PJ, Lumley TL, Pepe MS. Time-Dependent ROC Curves for Censored Survival Data and a Diagnostic Marker. Biometrics 2000;56:337-344.

25. R development core team. R: A language and environment for statistical computing. R Foundation for Statistical Computing. 2009.

26. Jack CR, Wiste HJ, Vemuri, P et al. Brain beta-amyloid measures and magnetic resonance imaging atrophy both predict time-to-progression from mild cognitive impairment to Alzheimer's disease. Brain 2010;133:3336-3348.

27. van Rossum IA, Vos S, Handels R, Visser PJ. Biomarkers as Predictors for Conversion from Mild Cognitive Impairment to Alzheimer-Type Dementia: Implications for Trial Design. J Alzheimers Dis 2010;20(3):881-891.

28. Elias-Sonnenschein LS, Viechtbauer W, Ramakers IH, Verhey FR, Visser PJ. Predictive value of APOE-e4 allele for progression from MCI to AD-type dementia: a meta-analysis. J Neurol Neurosurg Psychiatry 2011;82(10):1149-1156.

29. Leoni V. The effect of apolipoprotein E (ApoE) genotype on biomarkers of amyloidogenesis, tau pathology and neurodegeneration in Alzheimer's disease. Clin Chem Lab Med 2011;49:375-383.

30. Jungwirth S, Zehetmayer S, Hinterberger M, Tragl KH, Fischer P. The validity of amnestic MCI and non-amnestic MCI at age 75 in the prediction of Alzheimer's dementia and vascular dementia. Int Psychogeriatr 2012;24:959-966. 


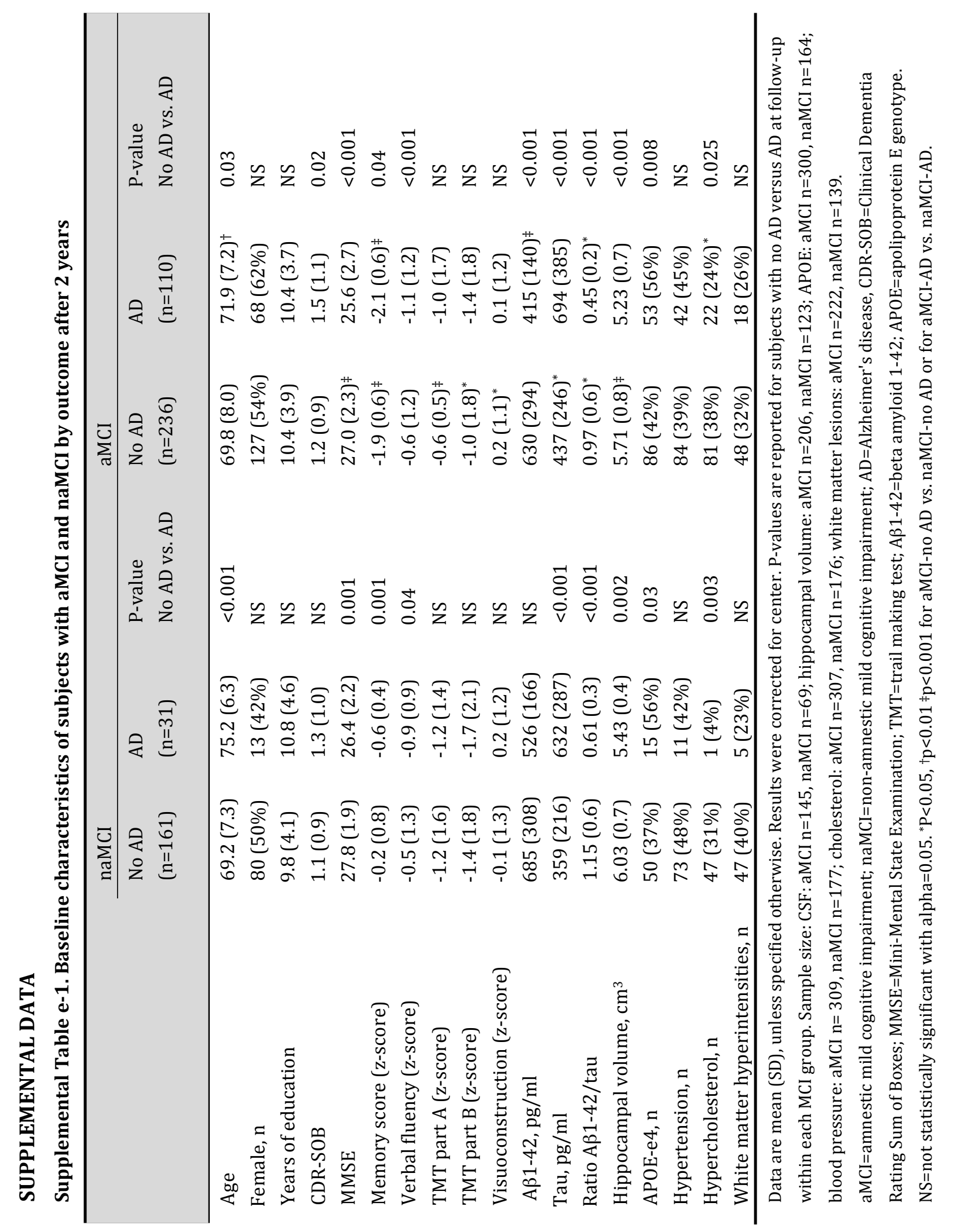




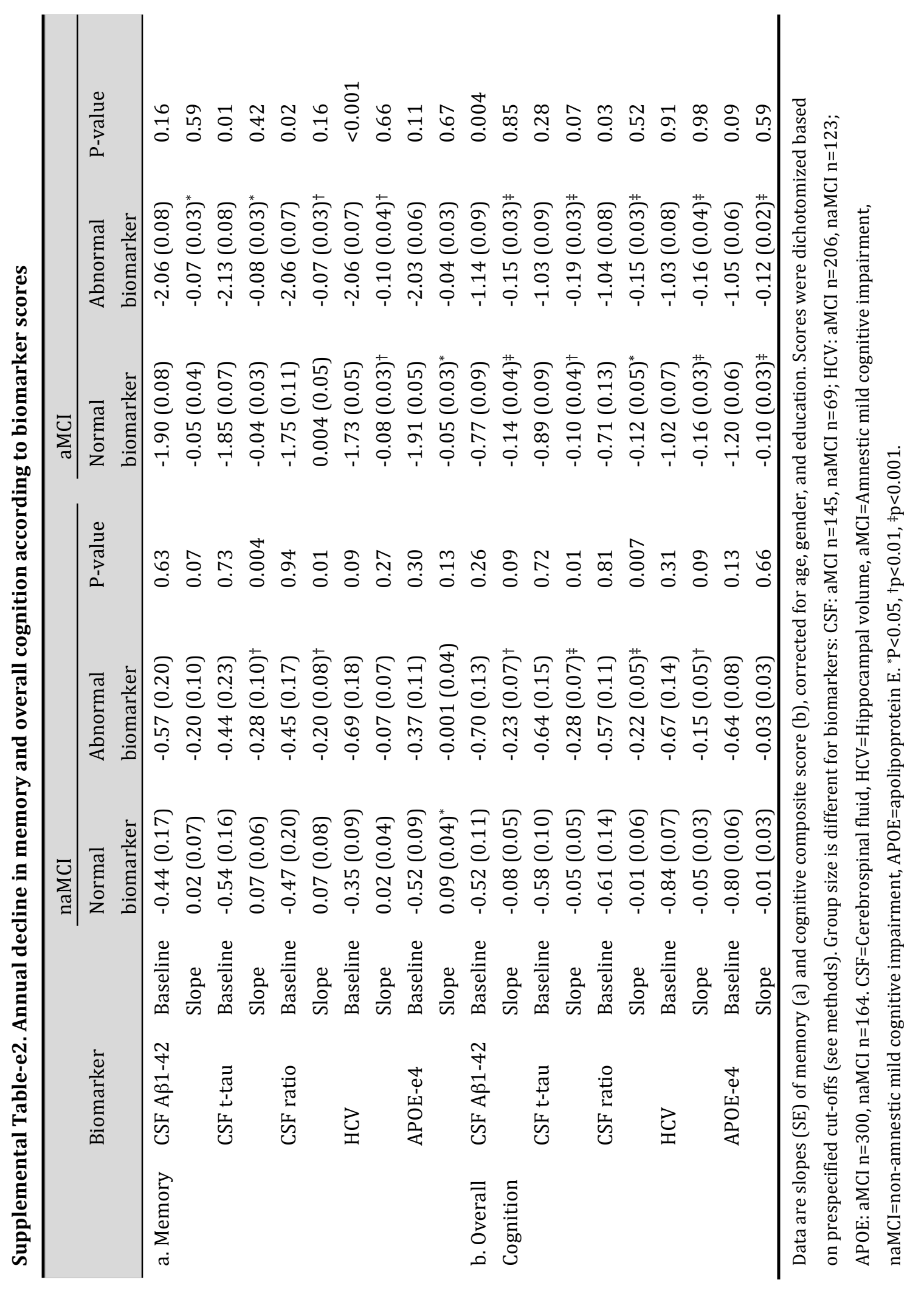




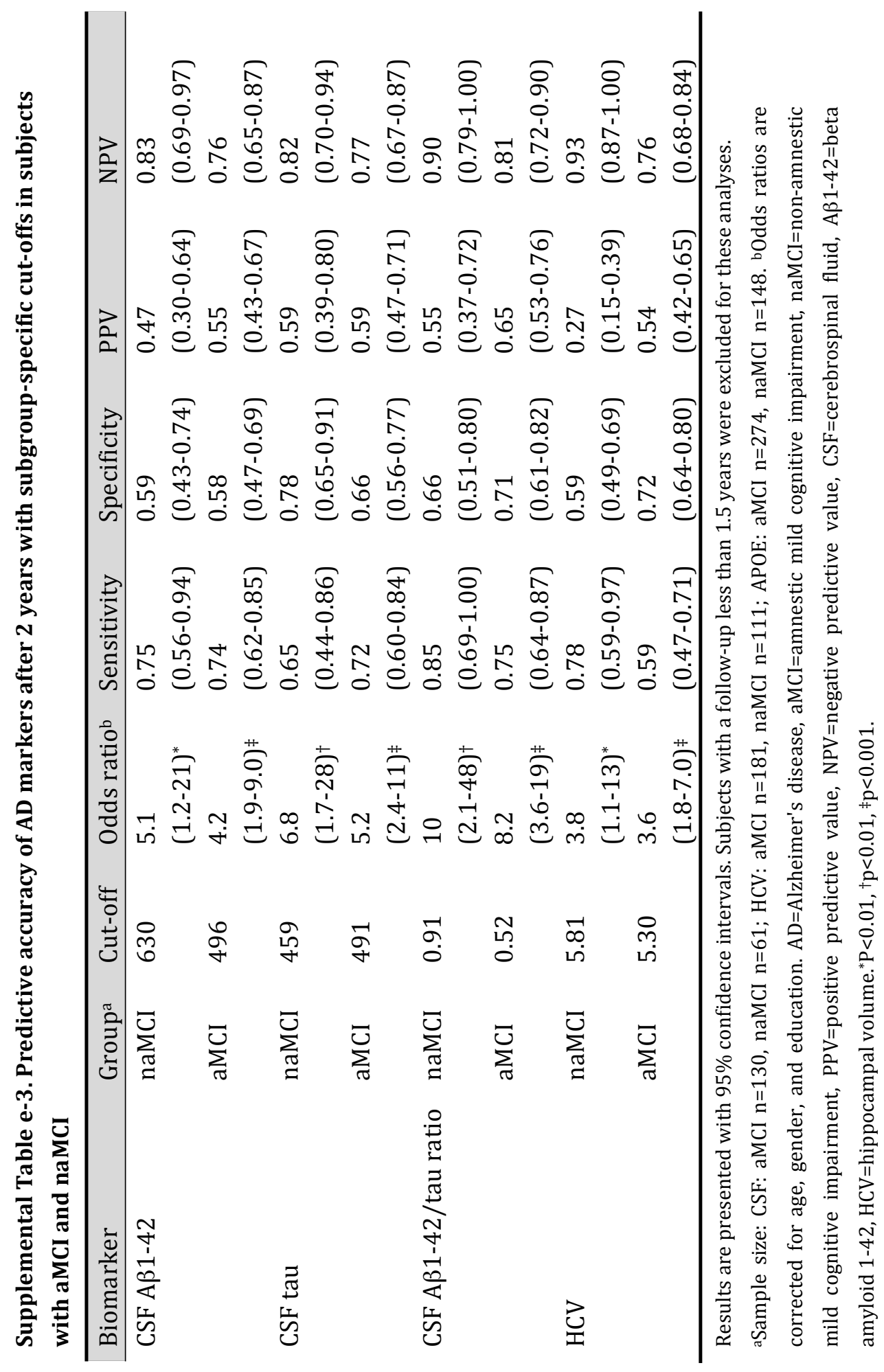





\title{
CHAPTER 5
}

\author{
INJURY MARKERS PREDICT TIME TO DEMENTIA IN \\ SUBJECTS WITH MCI AND AMYLOID PATHOLOGY
}

Neurology 2012;79:1809-16.

Ineke A. van Rossum, Stephanie J.B. Vos, Leah Burns, Dirk L. Knol, Philip Scheltens, Hilkka Soininen, Lars-Olof Wahlund, Harald Hampel, Magda Tsolaki, Lennart Minthon, Gilbert L'Italien, Wiesje M. van der Flier, Charlotte E. Teunissen, Kaj Blennow, Frederik Barkhof, Daniel Rueckert, Robin Wolz, Frans Verhey, Pieter Jelle Visser 


\section{ABSTRACT}

Objectives

Alzheimer's disease (AD) can now be diagnosed in subjects with mild cognitive impairment (MCI) using biomarkers. However, little is known about the rate of decline in those subjects. In this cohort study we aimed to assess the conversion rate to dementia and identify prognostic markers in subjects with MCI and evidence of amyloid pathology.

\section{Methods}

We pooled subjects from the VUmc Alzheimer Center and the DESCRIPA study. We included subjects with MCI, an abnormal level of Aß1-42 in the cerebrospinal fluid (CSF) and at least one diagnostic follow-up visit. We assessed the effect of APOE-genotype, CSF t-tau and p-tau and hippocampal volume on time to AD-type dementia using Cox proportional hazard models and on decline on the MMSE using linear mixed models.

\section{Results}

We included 110 MCI subjects with abnormal CSF Aß1-42 and a mean MMSE of 26.3 \pm 2.8 . During a mean follow up of $2.2 \pm 1.0$ (range $0.4-5.0$ ) years, 63 subjects (57\%) progressed to AD-type dementia. Abnormal CSF t-tau (hazard ratio (HR) 2.3, 95\% CI 1.1-4.6, p=0.03) and CSF p-tau (HR 3.5, 1.3-9.2, p=0.01) concentration and hippocampal atrophy (HR 2.5, 1.1-5.6, $\mathrm{p}=0.02$ ) predicted time to dementia. For subjects with both abnormal t-tau concentration and hippocampal atrophy HR was 7.3 (1.0-55.9, p=0.06). Furthermore, abnormal CSF t-tau and p-tau concentrations and hippocampal atrophy predicted decline in MMSE score.

\section{Conclusions}

In subjects with $\mathrm{MCI}$ and evidence of amyloid pathology, the injury markers CSF t-tau and ptau and hippocampal atrophy can predict further cognitive decline. 


\section{INTRODUCTION}

Recently, two sets of research criteriaa $^{1,2}$, were established allowing a diagnosis of Alzheimer's disease (AD) in subjects with mild cognitive impairment (MCI) and biomarker evidence of $\mathrm{AD}$ pathology. An international working group defined criteria for 'prodromal $\mathrm{AD}^{\prime}$ in $2007^{2}$ and in 2011 the National Institute of Aging and the Alzheimer Association published criteria for 'MCI due to $\mathrm{AD}^{\prime} .{ }^{1}$ However, at this moment, prognosis of subjects fulfilling these criteria is largely unknown, which limits the use of the criteria in clinical practice. Prognostic markers for cognitive decline in subjects with $\mathrm{MCI}$ due to $\mathrm{AD}^{1}$ or prodromal $\mathrm{AD}^{2}$ are therefore urgently needed.

Subjects can be diagnosed with $\mathrm{MCI}$ due to $\mathrm{AD}^{1}$ or prodromal $\mathrm{AD}^{2}$ when they have a clinical diagnosis of MCI and biomarker evidence of either amyloid-beta pathology, ADrelated neuronal injury or both. Abnormal amyloid markers may already be present at the earliest stage of the disease and reach a plateau in a very early stage of the disease and can therefore be useful as an early diagnostic marker. ${ }^{3-5}$ Markers of the subsequent neuronal injury on the other hand, such as cerebrospinal fluid (CSF) tau and hippocampal atrophy on MRI, may reflect more advanced pathology and might be useful as prognostic markers. ${ }^{3-5}$

For the present study we selected subjects with MCI and evidence of amyloid pathology, defined by an abnormal level of amyloid-beta 1-42 (Aß1-42) in the CSF. We hypothesized that the injury markers tau ( $\mathrm{t}$-tau) and phopshorylated tau (p-tau) ${ }^{6-8}$ in CSF and hippocampal atrophy on $\mathrm{MRI}^{9,10}$ would be associated with progression to AD-type dementia and cognitive decline.

\section{METHODS}

\section{Subjects}

We selected subjects from the DESCRIPA cohort and the memory clinic of the Alzheimer Center of the VU University medical center (VUmc). DESCRIPA is a European multicenter study performed in a memory clinic setting. ${ }^{11}$ The VUmc was one of the DESCRIPA partners and contributed an additional sample of subjects that were seen outside the DESCRIPA inclusion period. Inclusion criteria were a clinical diagnosis of MCI, an abnormal level of CSF Aß1-42, based on a clinically validated cut-off $(\leq 550 \mathrm{pg} / \mathrm{ml}),{ }^{12}$ and at least one follow up diagnosis. Subjects with obvious causes for MCI other than AD, such as alcohol abuse or severe depression, were excluded. In 10 of the participating centers CSF was collected. Of the subjects enrolled at these centers between 2003 and 2005, 64 subjects fulfilled inclusion criteria. From the VUmc 46 additional subjects were included.

\section{Standard protocol approvals, registrations and patient consents}

The medical ethics committee at each center approved the study. All patients provided written informed consent. 


\section{Clinical assessment}

Diagnosis of MCI was made according to the criteria of Petersen. ${ }^{13}$ Raw scores on neuropsychological tests were corrected for age, gender and educational level in accordance with locally collected or published normative data and expressed as z-scores. MCI was defined as a z-score below -1.5 SD on any of the following tests: the learning measure or delayed recall of a verbal memory task, trail making test (TMT) part A, TMT part B, verbal fluency or Rey figure copy or equivalent test, as described in more detail previously. ${ }^{11,14}$ Follow-up assessment was performed annually up to 5 years. For subjects from the Alzheimer center of the VUmc, follow up was part of regular patient care. Diagnosis of ADtype dementia was made according to the DSM-IV ${ }^{15}$ and NINCDS-ADRDA criteria. ${ }^{16}$ Time to dementia was defined as the time between baseline visit and the date AD-type dementia was diagnosed.

\section{CSF analyses}

CSF was collected by lumbar puncture, centrifuged, and stored at $-80^{\circ} \mathrm{C}$ in polypropylene tubes. One sample was thawed twice but analyses without this sample revealed similar results. CSF A $31-42$, t-tau and tau phosphorylated at threonine 181 (p-tau) were measured with Innotest sandwich ELISA (Innogenetics, Ghent, Belgium) in Gothenburg for the DESCRIPA cohort and in Amsterdam for the VUmc cohort. We corrected for interlaboratory ELISA differences by means of 33 samples that were analysed at both labs and adjusted VUmc values to those of DESCRIPA using the equating formula: Gothenborg=(SD Gothenborg/SD VUmc)*VUmc+average Gothenborg-((SD Gothenborg/SD VUmc)*average VUmc). ${ }^{17}$

\section{MRI analyses}

For the DESCRIPA cohort, subjects were scanned according to the routine MRI protocol at each site. Scanning was performed at 1.0 or $1.5 \mathrm{~T}$ and included a three-dimensional $\mathrm{T} 1$ weighted gradient echo sequence with near-isotropic voxels and a fast fluid attenuated inversion recovery (FLAIR) sequence. ${ }^{14,18}$

Hippocampal volume (HCV) was measured at the Department of Computing of Imperial College London, using LEAP, a segmentation technique based on atlas registration. ${ }^{19}$ We tested whether the MRI field strength influenced the LEAP scores in 348 subjects with MCI from the Descripa cohort. Field strength did not affect the LEAP score (difference of $0.07 \%$, p-value $=0.8$ after correction for age, gender, educational level, baseline MMSE score and follow up diagnosis) and therefore we used data from both field strengths without correction.

MRI data were available in 35 of the 64 subjects (55\%) from the DESCRIPA cohort and in 30 of the 46 subjects (65\%) from VUmc. Subjects with and without MRI data available did not differ with respect to age, gender, educational level, APOE status, CSF markers or score on the Mini-Mental State Examination (MMSE) ${ }^{20}$ at baseline. 


\section{APOE genotyping}

DNA was isolated from $10 \mathrm{ml}$ EDTA blood for apolipoprotein E (APOE) genotyping, using the light cycler APOE mutation detection kit (Roche Diagnostics GmbH, Mannheim, Germany). APOE genotype was determined in 99 subjects (90\%). Subjects in whom no APOE status was determined scored higher on the MMSE at baseline (27.7 vs 26.1, $\mathrm{p}=0.005$ ). There were no differences with respect to age, gender, educational level, medial temporal lobe atrophy or CSF markers between subjects with and without APOE data available. Subjects were classified as APOE- $\varepsilon 4$ positive when having one or two APOE- $\varepsilon 4$ alleles.

\section{Statistical analyses}

Analyses were performed with SPSS 18.0 for the Macintosh.

For group comparisons of subjects with and without AD-type dementia at follow up we used chi-squared tests for categorical variables and Student's t-tests for continuous variables. Data of the CSF markers were log transformed in order to obtain an approximately normal distribution. For further analyses we used dichotomized values of the respective markers. We used clinically validated cut-off points for CSF t-tau $(>375 \mathrm{pg} / \mathrm{ml})$ and p-tau $(>52$ $\mathrm{pg} / \mathrm{ml}){ }^{12}$ For hippocampal volume we used a summed volume of the left and right hippocampus of $5.39 \mathrm{~cm}^{3}$ as cut-off point. This cut-off point could best differentiate between healthy controls and subjects with AD-type dementia in the ADNI cohort (Vos et al., submitted), based on the Youden index using R. ${ }^{21,22}$ This cut-off point was similar to the cutoff point of $5.34 \mathrm{~cm}^{3}$ that could best predict AD-type dementia in our own dataset. ${ }^{23}$

We assessed the effect of APOE genotype, CSF levels of t-tau and p-tau, hippocampal atrophy on time to dementia using Cox proportional hazards with correction for age, gender, education and MMSE score at baseline. Analyses were performed for each variable alone and with all variables together using a stepforward model to select the variables that could best predict AD-type dementia.

We also assessed the association of CSF t-tau and p-tau and hippocampal volume with decline in MMSE score. We performed mixed models analyses with an unstructured covariance structure with correction for age, gender, educational level and center. ${ }^{24}$

\section{RESULTS}

\section{Baseline characteristics}

We included 110 subjects with MCI and abnormal CSF Aß1-42. Subjects were on average \pm standard deviation (SD) $70.8 \pm 7.7$ years old, $46 \%$ was female and $62 \%$ had at least 1 APOE- $\varepsilon 4$ allele. Mean MMSE score was $26.3 \pm 2.8$. Baseline characteristics of the subjects are shown in Table 1. Two subjects progressed to other types of dementia (one subject with vascular dementia and one subject with Parkinsons disease dementia). They were included in the group of subjects that did not progress to AD-type dementia. Excluding those two subjects from the analyses did not change the results (data not shown). 
Table 1. Baseline characteristics according to diagnosis at follow up

\begin{tabular}{|c|c|c|c|}
\hline & All subjects & $\begin{array}{l}\text { No AD-type } \\
\text { dementia at follow } \\
\text { up }\end{array}$ & $\begin{array}{l}\text { AD-type } \\
\text { dementia at } \\
\text { follow up }\end{array}$ \\
\hline $\mathrm{N}$ & 110 & 47 & 63 \\
\hline Age & $70.8 \pm 7.7$ & $70.1 \pm 8.1$ & $71.3 \pm 7.4$ \\
\hline Female, n (\%) & $51(46)$ & $20(43)$ & $31(49)$ \\
\hline Education, years & $10.8 \pm 3.5$ & $10.5 \pm 3.5$ & $11.1 \pm 3.4$ \\
\hline Follow up, years & $2.2 \pm 1.0$ & $2.3 \pm 1.1$ & $2.0 \pm 0.9$ \\
\hline APOE- $\varepsilon 4$ positive, $n(\%)^{\$}$ & $61(62)$ & $23(54)$ & $38(68)$ \\
\hline $\mathrm{A} \beta 1-42, \mathrm{pg} / \mathrm{ml}$ & $382 \pm 98$ & $369 \pm 100$ & $392 \pm 97$ \\
\hline T-tau, pg/ml & $564 \pm 345$ & $421 \pm 252$ & $670 \pm 368^{\#}$ \\
\hline T-tau, abnormal*, n (\%) & $81(74)$ & $28(60)$ & $53(84)^{\#}$ \\
\hline P-tau, pg/ml & $89 \pm 49$ & $71 \pm 35$ & $103 \pm 54^{\#}$ \\
\hline P-tau, abnormal*, n (\%) & $90(82)$ & $32(68)$ & $58(92)^{\#}$ \\
\hline Hippocampal volume, $\mathrm{cm}^{3 \dagger}$ & $5.4 \pm 0.7$ & $5.8 \pm 0.8$ & $5.2 \pm 0.6^{\#}$ \\
\hline Hippocampal atrophy\&, n (\%) & $35(54)$ & $8(31)$ & $27(69)^{\#}$ \\
\hline MMSE score & $26.3 \pm 2.8$ & $26.8 \pm 2.6$ & $25.9 \pm 2.8$ \\
\hline $\begin{array}{l}\text { Verbal memory, learning } \\
\text { (z-score) }\end{array}$ & $-1.5 \pm 1.0$ & $-1.4 \pm 1.1$ & $-1.6 \pm 0.9$ \\
\hline $\begin{array}{l}\text { Verbal memory, delayed recall } \\
\text { (z-score) }\end{array}$ & $-1.6 \pm 1.0$ & $-1.3 \pm 1.1$ & $-1.9 \pm 0.8^{\#}$ \\
\hline Verbal fluency (z-score) & $-0.8 \pm 1.1$ & $-0.7 \pm 1.3$ & $-1.0 \pm 0.9$ \\
\hline TMT part A (z-score) & $-0.8 \pm 1.8$ & $-0.7 \pm 1.6$ & $-0.9 \pm 2.0$ \\
\hline TMT part B (z-score) & $-1.1 \pm 1.6$ & $-1.0 \pm 1.6$ & $-1.2 \pm 1.6$ \\
\hline Visuoconstruction (z-score) & $0.2 \pm 1.1$ & $0.03 \pm 1.2$ & $0.3 \pm 1.0$ \\
\hline
\end{tabular}

Data are mean \pm standard deviation unless otherwise indicated. \$APOE genotype was determined in 99 subjects, thippocampal volume was determined in 65 subjects, ${ }^{*}$ abnormal values were defined as $>375 \mathrm{pg} / \mathrm{ml}$ for CSF t-tau and $>52 \mathrm{pg} / \mathrm{ml}$ for CSF p-tau, \&hippocampal atrophy was defined as a summed volume of left and right hippocampus of $<5.39 \mathrm{~cm}^{3}$. \# $\mathrm{p}<0.005$ compared to no dementia at follow up, $\neq \mathrm{p}<0.05$ compared to no dementia at follow up. APOE=apolipoprotein $\quad E, \quad C S F=$ cerebrospinal fluid, $A \beta 1-42=$ beta amyloid1-42, $t$-tau=total tau, $p$-tau=tau phosphorylated at threonine 181, MMSE=Mini-Mental State Examination.

\section{Predictors of progression to AD-type dementia}

During a mean follow up of $2.2 \pm 1.0$ years (median 2.0 years, range $0.4-5.0$ years) 63 subjects (57\%) progressed to AD-type dementia. These subjects had higher levels of CSF t-tau (mean \pm SD $670 \pm 368$ vs $421 \pm 252 \mathrm{pg} / \mathrm{ml}, \mathrm{p}<0.001$ ) and p-tau $(103 \pm 54$ vs $71 \pm 35 \mathrm{pg} / \mathrm{ml}$, $\mathrm{p}<0.001)$, a smaller hippocampal volume $\left(5.2 \pm 0.6 \mathrm{~cm}^{3}\right.$ vs $\left.5.8 \pm 0.8 \mathrm{~cm}^{3}, \mathrm{p}=0.002\right)$ and a lower score on the delayed recall of a verbal memory task ( $\mathrm{z}$-score $-1.9 \pm 0.8$ vs $-1.3 \pm 1.1, \mathrm{p}=0.004$ ) than subjects that did not progress to AD-type dementia (Table 1).

\section{Predictors of time to AD-type dementia}

Survival analyses using Cox proportional hazards model with correction for age, gender and education showed that time to dementia was predicted by abnormal CSF t-tau (hazard ratio 
(HR) 2.3, 95\% CI 1.1-4.6, p=0.03), abnormal CSF p-tau (HR 3.5, 1.3-9.2, p=0.01) and hippocampal atrophy (HR 2.5, 1.1-5.6, p=0.02, Figure 1, Table e-1). After correction for baseline MMSE score results remained essentially the same, with an HR of 2.0 (1.0-4.2, $\mathrm{p}=0.06)$ for CSF t-tau, $3.1(1.2-8.4, \mathrm{p}=0.03)$ for CSF $\mathrm{p}$-tau and $2.2(1.0-5.0, \mathrm{p}=0.06)$ for hippocampal atrophy. Of the neuropsychological measures only delayed recall predicted AD-type dementia (HR 2.1 (1.0-4.3), $\mathrm{p}=0.05$, Table e-1). The APOE- $\varepsilon 4$ genotype, age, gender and education did not predict time to dementia (Table e-1). Cox multivariate analyses with forward-step selection and biomarkers entered as log-transformed continuous variables selected only CSF p-tau ( $\beta$ 1.2, HR 3.3 (1.4-7.5) p=0.005). In the multivariate analysis we did not find a significant interaction between CSF p-tau or t-tau with hippocampal atrophy $(\mathrm{p}=0.8)$.

\section{MMSE slope analyses}

Subjects with abnormal CSF t-tau more rapidly declined on the MMSE, with an annual decline of -1.1, compared to -0.4 for subjects with normal CSF t-tau (Table 2). At baseline there were no differences in MMSE score between subjects with normal and abnormal CSF ttau (26.5 and 26.3 respectively). For CSF p-tau results were similar (Table 2). Subjects with hippocampal atrophy showed more rapid decline in MMSE score compared to subjects without hippocampal atrophy (average annual decline -1.2 vs $-0.5, p=0.09$ ) (Table 2). At baseline, subjects with hippocampal atrophy had lower MMSE score than subjects without hippocampal atrophy (25.6 vs 27.0 , p=0.02).

\section{Biomarker subgroup analyses}

In order to investigate the effect of the combination of abnormal CSF t-tau and hippocampal atrophy on progression to AD-type dementia and cognitive decline, we subdivided subjects with both CSF and MRI available ( $\mathrm{N}=65)$ into three groups, depending on their biomarker status at baseline (Figure e-1): 1. normal CSF t-tau and no hippocampal atrophy ( $\mathrm{N}=9$, of whom 1 progressed to AD-type dementia), 2. either abnormal CSF t-tau or hippocampal atrophy ( $\mathrm{N}=28$, of whom 16 progressed to AD-type dementia), 3. both abnormal CSF t-tau and hippocampal atrophy ( $\mathrm{N}=28$, of whom 22 progressed to AD-type dementia). Compared to subjects with normal CSF t-tau and no hippocampal atrophy, subjects with either abnormal CSF t-tau or hippocampal atrophy had an HR (95\% CI) of $5.2(0.7-40.3, p=0.1)$ for progression to AD-type dementia. For subjects with both abnormal CSF t-tau and hippocampal atrophy the HR was $7.3(1.0-55.9, \mathrm{p}=0.06)$ (Table 3). 
Table 2. Predictors for decline in MMSE score

\begin{tabular}{llllccc}
\hline & & $\mathrm{N}$ & Baseline MMSE & p-value \pm & Slope & p-value $^{ \pm}$ \\
\hline CSF t-tau & $\geq 375 \mathrm{pg} / \mathrm{ml}$ & 81 & $26.2(25.1-27.4)$ & 0.6 & $-1.1(-1.4--0.8)$ & 0.02 \\
& $<375 \mathrm{pg} / \mathrm{ml}$ & 29 & $26.5(25.3-27.9)$ & & $-0.4(-0.9-0.2)$ & \\
CSF p-tau & $\geq 52 \mathrm{pg} / \mathrm{ml}$ & 90 & $26.2(25.1-27.3)$ & 0.4 & $-1.1(-1.3--0.8)$ & 0.005 \\
& $<52 \mathrm{pg} / \mathrm{ml}$ & 20 & $26.7(25.3-28.2)$ & & $-0.04(-0.7-0.6)$ & \\
Hippocampal & $<5.39 \mathrm{~cm}^{3}$ & 35 & $25.6(23.8-27.5)$ & 0.02 & $-1.2(-1.5--0.8)$ & 0.01 \\
volume & $\geq 5.39 \mathrm{~cm}^{3}$ & 30 & $27.0(25.2-28.8)$ & & $-0.5(-0.9--0.1)$ & \\
\hline
\end{tabular}

Baseline MMSE-scores and slope values of annual change in MMSE-score estimated using mixed models with correction for age, gender, educational level and center. Values are estimated assuming subjects are $50 \%$ female, 70 years of age and with 11 years of education. Data are mean (95\% confidence interval). ${ }^{ \pm} \mathrm{p}$-value of the difference between subjects with normal and abnormal values for each biomarker. CSF=cerebrospinal fluid, t-tau=total tau, p-tau=tau phosphorylated at threonine 181, MMSE=Mini-Mental State Examination.

Table 3. Progression to AD-type dementia and rate of cognitive decline with respect to biomarker status at baseline

\begin{tabular}{lcllll}
\hline $\begin{array}{l}\text { CSF t-tau and } \\
\text { hippocampal } \\
\text { volume* }\end{array}$ & $\mathrm{N}$ & $\begin{array}{l}\text { Dementia free } \\
\text { survival after } 4 \\
\text { years }\end{array}$ & HR dementia & Baseline MMSE\$ & Slope \\
\hline Both normal & 9 & $0.73 \pm 0.06$ & reference & $27.4(25.2-29.6)$ & $-0.1(-0.9-0.7)$ \\
One abnormal & 28 & $0.19 \pm 0.08$ & $5.2(0.7-40.3)$ & $26.6(24.7-28.5)$ & $-0.8(-1.2--0.4)^{\#}$ \\
Both abnormal & 28 & $0.09 \pm 0.03$ & $7.3(1.0-55.9)$ & $25.5(23.6-27.4)$ & $-1.1(-1.5--0.7)^{ \pm}$ \\
\hline
\end{tabular}

Dementia free survival and the hazard ratio were calculated using Cox regression analyses with correction for age, gender and educational level. Baseline MMSE-scores and slope values of annual change in MMSE-score estimated using mixed models with correction for age, gender, educational level and center. Values are estimated assuming subjects are 50\% female, 71 years of age and with 11 years of education. Data are mean (95\% confidence interval) or mean \pm SE. *Abnormal CSF t-tau was defined as a value $>375 \mathrm{pg} / \mathrm{ml}$, Hippocampal atrophy was defined as a volume of both left and right hippocampus of $<5.39 \mathrm{~cm}^{3}$. \$ Differences in baseline MMSE between the groups were not statistically significant. \#p-value compared to both markers normal=0.1, ${ }^{ \pm} p$-value compared to both markers normal=0.02. $\mathrm{CSF}=$ cerebrospinal fluid, $\mathrm{t}$-tau=total tau, HR=hazard ratio, MMSE=MiniMental State Examination, SE=standard error. 


\section{A CSF t-tau}

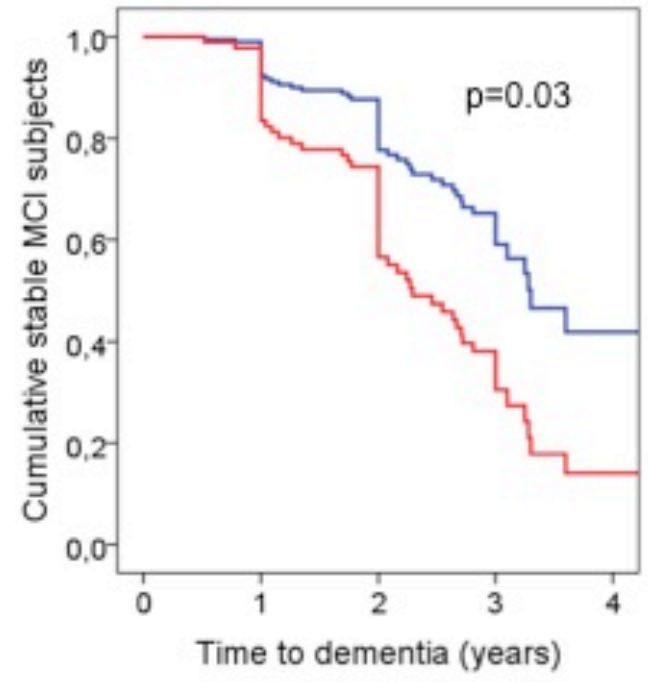

1.C Hippocampal atrophy

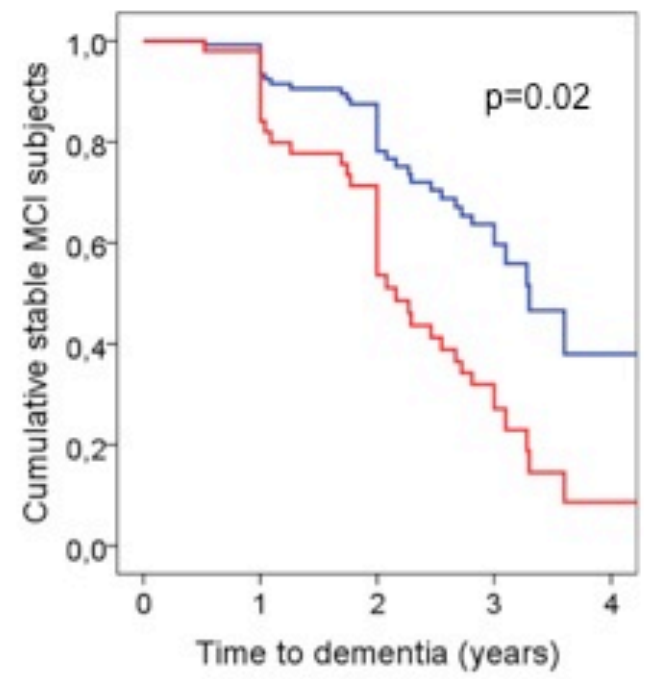

1.B CSF p-tau

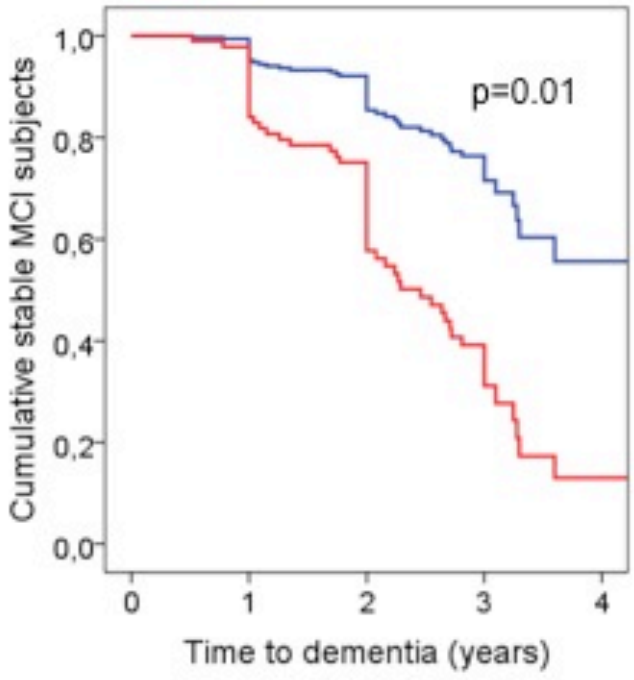

Figure 1. Survival curves for time to dementia in subjects with MCI and abnormal CSF Aß1-42, corrected for age, gender and education

Red lines indicate the subjects with an abnormal value of each respective marker, defined as CSF t-tau $>375 \mathrm{pg} / \mathrm{ml}$ (A), CSF p-tau $>52 \mathrm{pg} / \mathrm{ml}$ (B) and hippocampal volume $<5.39 \mathrm{~cm}^{3}$ (C). Blue lines indicate the subjects with normal values of each marker. $\mathrm{MCI}=$ mild cognitive impairment, $\mathrm{CSF}=$ cerebrospinal fluid, $\mathrm{A} \beta 1-42=$ beta amyloid1-42, $\mathrm{t}$ tau=total tau, p-tau=tau phosphorylated at threonine 181. 


\section{A. CSF t-tau}

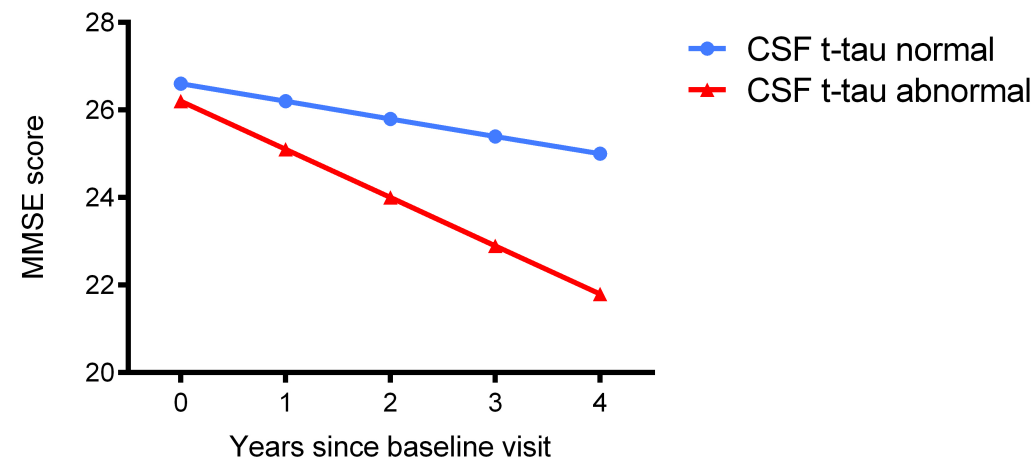

B. Hippocampal volume

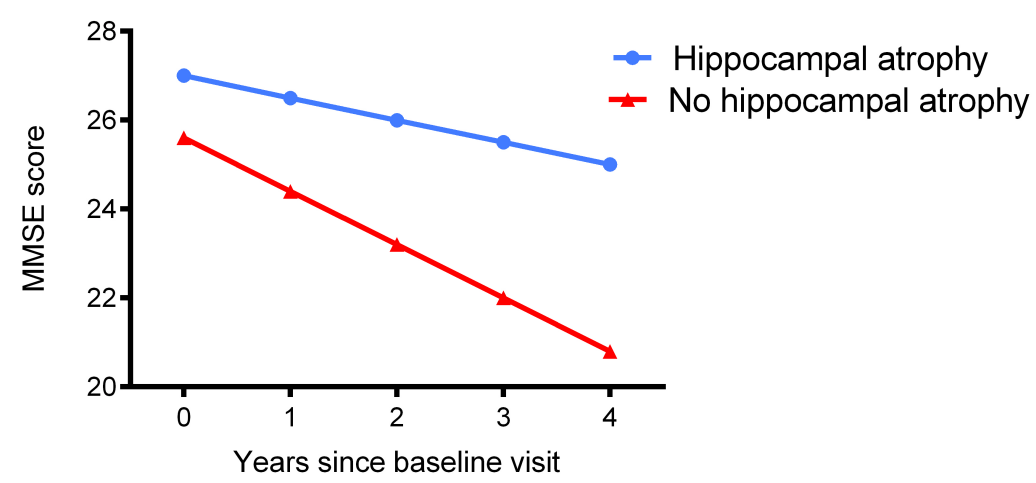

C. Combination of CSF t-tau and hippocampal volume

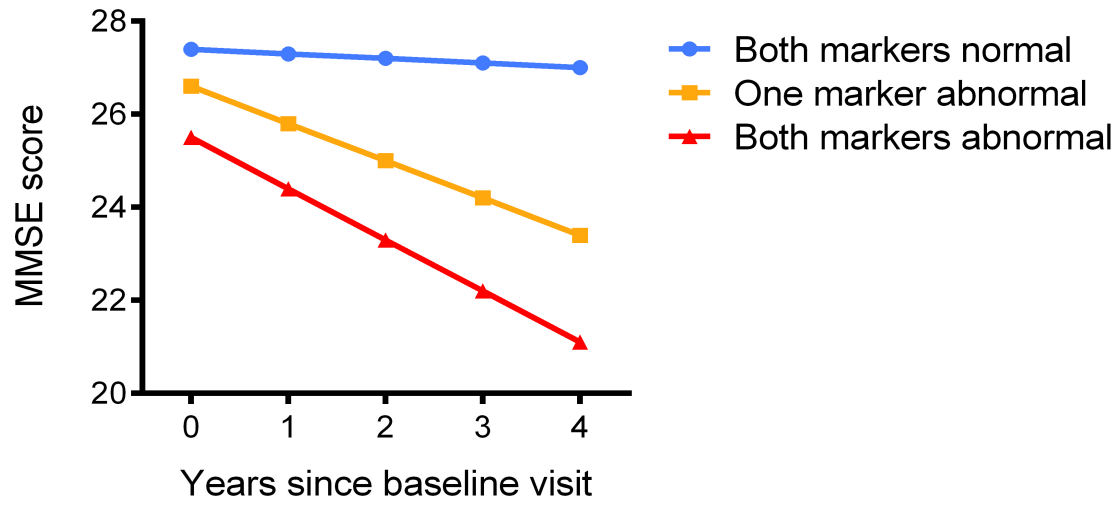

Figure 2. Decline in MMSE score in subjects with MCI and abnormal CSF Aß1-42 according to CSF ttau and hippocampal volume

Slopes of decline in MMSE score in subjects with MCI and abnormal CSF Aß1-42. Subjects were classified according to their CSF t-tau levels and hippocampal volume at baseline. Abnormal values were defined as CSF tau $>375 \mathrm{pg} / \mathrm{ml}$ and hippocampal volume $<5.39 \mathrm{~cm}^{3}$. Figure A+B: the blue (dot) line indicates the subjects with normal values. The red (triangle) line indicates subjects with abnormal values. Figure C: the blue (dot) line indicates the subjects with normal values of both. The red (triangle) line indicates subjects with both markers abnormal. The orange (square) line indicates subjects with either abnormal CSF t-tau or hippocampal atrophy. CSF=cerebrospinal fluid, $\mathrm{t}$-tau=total tau, MMSE=Mini-Mental State Examination. 
The annual decline in MMSE score was -0.1 (p-value slope=0.8) for subjects with normal CSF t-tau and no hippocampal atrophy, $-0.8(\mathrm{p}=0.001)$ for subjects with either abnormal CSF $\mathrm{t}$ tau or hippocampal atrophy and $-1.1(\mathrm{p}<0.001)$ for subjects with both abnormal CSF t-tau and hippocampal atrophy (Table 3, Figure 2). The slopes of decline of subjects with one or two abnormal markers differed from the slope of subjects with both markers normal, but not from each other. For subjects with only abnormal CSF t-tau $(\mathrm{N}=21)$ the annual decline in MMSE score was $-0.6(-1.0--0.2, \mathrm{p}=0.006)$. For subjects with only hippocampal atrophy, no slope analyses could be performed, due to small sample size $(\mathrm{N}=7)$.

\section{DISCUSSION}

In this prospective study of subjects who fulfilled the criteria for 'MCI due to $\mathrm{AD}^{1}$ and 'prodromal $\mathrm{AD}^{2}$ based on abnormal CSF Aß1-42, we found that during a mean follow up of 2.2 years 63 subjects (57\%) progressed to AD-type dementia. High CSF levels of t-tau and ptau and hippocampal atrophy predicted progression to dementia and decline in MMSE score.

The overall annual conversion rate to dementia of around $20 \%$ in this study was higher than the conversion rate typically observed in subjects with MCI unselected for biomarkers status. ${ }^{25}$ For comparison, subjects with MCI and a normal concentration of CSF Aß1-42 in our dataset had an annual conversion rate of less than $10 \%$ (data not shown). Still, a considerable percentage of our subjects did not develop AD-type dementia within the follow up period. Since abnormal beta amyloid is suggested to be an early marker for $\mathrm{AD}^{3}$, higher progression rates to AD-type dementia might be expected with a longer follow up period.

The rapid decline to dementia in subjects with high CSF levels of t-tau and p-tau and hippocampal atrophy could mean that these subjects either suffered from a more aggressive course of the disease or were already in a more advanced stage when assessed at baseline. Slope analyses suggested that they suffered from a more aggressive course of the disease since they showed a more rapid decline in MMSE score compared to subjects with normal values of these markers at baseline. This is in line with previous studies that found a more rapid cognitive decline in subjects with AD-type dementia with high levels of CSF tau. ${ }^{26,27}$ Subjects with hippocampal atrophy may have also been already in a more advanced stage of the disease at baseline as they had lower MMSE scores at baseline compared to subjects without hippocampal atrophy. This is consistent with the previously suggested order of events in the amyloid cascade, ${ }^{3,4}$ with hippocampal atrophy being a relatively late feature of AD pathology. In a previous study in subjects with MCI and biomarker evidence of beta amyloid pathology, hippocampal atrophy also predicted time to dementia. ${ }^{28}$ In another study in subjects with MCI who all progressed to AD-type dementia, CSF t-tau, CSF p-tau and hippocampal atrophy were also associated with rapid progression from MCI to AD-type dementia, while CSF Aß1-42 was not. ${ }^{5}$ Our finding that the predictive value of the respective CSF and MRI markers for progression to AD-type dementia remained after correction for 
baseline MMSE score indicates that AD-biomarkers can have prognostic value in addition to clinical measures alone.

The predictive accuracy of CSF t-tau and p-tau and hippocampal atrophy we observed in our MCI subjects with abnormal CSF Aß1-42 was lower than that reported in studies conducted in subjects with MCI regardless of amyloid biomarker status ${ }^{7-9,23}$ Most likely this is because in our analyses only the additional predictive effect relative to abnormal amyloid was tested, although differences could partly also be due to differences in setting and other study characteristics.

We found no differences in age, gender and APOE status between subjects with and without dementia at follow up, although age, gender and APOE genotype are known risk factors for $\mathrm{AD}$ in the general population. A possible explanation for this finding could be that advanced age and APOE- $\varepsilon 4$ genotype are risk factors for development of abnormal beta amyloid processing, but do not influence clinical progression once abnormal beta amyloid processing is established.

We included subjects with MCI and abnormal amyloid. According to the criteria of the National Institute of Aging and the Alzheimer Association, ${ }^{1}$ these subjects would meet criteria of 'MCI due to AD-intermediate likelihood'. Of the 65 subjects with both CSF and MRI available 9 subjects (14\%) had both normal CSF t-tau and normal hippocampal volume and met criteria of 'MCI, biomarker evidence uninformative'. The course of the disease in these subjects was relatively benign with a $27 \%$ conversion rate to AD-type dementia after 4 years, although the interpretation is limited by the small sample size. 28 subjects (43\%) had both abnormal CSF t-tau and hippocampal atrophy and fulfilled criteria for 'MCI due to AD, high likelihood'1. Their prognosis was poor, with 91\% progressing to AD-type dementia after 4 years. In 28 subjects (43\%), the injury markers were conflicting, with either CSF t-tau abnormal or hippocampal volume abnormal. According to the NIA-AA criteria it is not clear whether these subjects should be diagnosed as 'MCI, biomarker evidence uninformative' or 'MCI due to AD high likelihood'. ${ }^{1}$ Our data suggest that these subjects should be considered as 'MCI due to AD-high likelihood' since the decline in MMSE score and progression rate to AD-type dementia (81\%) was similar to that of subjects with both markers abnormal while the rate of decline on the MMSE was worse than that of subjects with both markers normal, although group comparisons are hampered by the small sample size.

Two subjects included in the study progressed to other types of dementia, despite abnormal CSF A $\beta 1-42$ levels ate baseline. One subject, aged 75, had extrapyramidal signs at baseline and was later diagnosed with Parkinson's disease dementia. CSF Aß1-42 was 326 $\mathrm{pg} / \mathrm{ml}$, CSF t-tau and CSF p-tau were normal and hippocampal volume was not available. Decreased CSF Aß1-42 has been described before in subjects with alphasynucleiopathies. ${ }^{29}$ This highlights the importance of ruling out causes for the cognitive symptoms other than AD before the criteria for MCI due to AD can be applied. ${ }^{1}$ The other subject, aged 61, was diagnosed with vascular dementia at follow up. She had a CSF Aß1-42 concentration of 357 $\mathrm{pg} / \mathrm{ml}$, and abnormal CSF t-tau and p-tau concentrations. On the MRI scan she had multiple 
vascular white matter lesions and parietal atrophy, in the absence of hippocampal atrophy. In retrospect this subject may have suffered from mixed dementia with both vascular and Alzheimer's pathology.

A major limitation of our study is that we did not have MRI data available of all subjects, which limited possibilities for multivariate analyses. Another limitation is the limited follow up. Studies with longer clinical follow up are needed to assess whether all subjects with MCI due to AD will indeed develop dementia eventually.

Our results implicate that markers of AD-related neuronal injury as CSF level of t-tau, p-tau and hippocampal atrophy, could help to identify those subjects with MCI due to AD that will more rapidly progress to dementia. Subjects with both abnormal CSF A $\beta 1-42$ and abnormal injury markers, thereby fulfilling criteria for 'MCI due to AD-high likelihood', showed most rapid cognitive decline and a high progression rate to AD-type dementia, even within our limited follow up period. 


\section{REFERENCES}

1. Albert MS, Dekosky ST, Dickson D, et al. The diagnosis of mild cognitive impairment due to Alzheimer's disease: Recommendations from the National Institute on Aging-Alzheimer's Association workgroups on diagnostic guidelines for Alzheimer's disease. Alzheimers Dement 2011;7:270-279.

2. Dubois B, Feldman HH, Jacova C, et al. Research criteria for the diagnosis of Alzheimer's disease: revising the NINCDS-ADRDA criteria. Lancet Neurol 2007;6:734-746.

3. Jack CR, Jr., Knopman DS, Jagust WJ, et al. Hypothetical model of dynamic biomarkers of the Alzheimer's pathological cascade. Lancet Neurol 2010;9:119-128.

4. Jack CR, Jr., Vemuri P, Wiste HJ, et al. Evidence for ordering of Alzheimer disease biomarkers. Arch Neurol 2011;68:1526-1535.

5. van Rossum IA, Visser PJ, Knol DL, et al. Injury Markers but not Amyloid Markers are Associated with Rapid Progression from Mild Cognitive Impairment to Dementia in Alzheimer's Disease. J Alzheimers Dis 2012.

6. Hampel H, Teipel SJ, Fuchsberger T, et al. Value of CSF beta-amyloid1-42 and tau as predictors of Alzheimer's disease in patients with mild cognitive impairment. Mol Psychiatry 2004;9:705-710.

7. Hansson O, Zetterberg H, Buchhave P, Londos E, Blennow K, Minthon L. Association between CSF biomarkers and incipient Alzheimer's disease in patients with mild cognitive impairment: a followup study. Lancet Neurol 2006;5:228-234.

8. Herukka SK, Hallikainen M, Soininen H, Pirttila T. CSF Abeta42 and tau or phosphorylated tau and prediction of progressive mild cognitive impairment. Neurology 2005;64:1294-1297.

9. Korf ES, Wahlund LO, Visser PJ, Scheltens P. Medial temporal lobe atrophy on MRI predicts dementia in patients with mild cognitive impairment. Neurology 2004;63:94-100.

10. Jack CR, Jr., Petersen RC, Xu YC, et al. Prediction of AD with MRI-based hippocampal volume in mild cognitive impairment. Neurology 1999;52:1397-1403.

11. Visser PJ, Verhey FR, Boada M, et al. Development of screening guidelines and clinical criteria for predementia Alzheimer's disease. The DESCRIPA Study. Neuroepidemiology 2008;30:254-265.

12. Mulder C, Verwey NA, van der Flier WM, et al. Amyloid-beta(1-42), total tau, and phosphorylated tau as cerebrospinal fluid biomarkers for the diagnosis of Alzheimer disease. Clin Chem 2010;56:248-253.

13. Petersen RC, Smith GE, Waring SC, Ivnik RJ, Tangalos EG, Kokmen E. Mild cognitive impairment: clinical characterization and outcome. Arch Neurol 1999;56:303-308.

14. Bouwman FH, Schoonenboom SN, van der Flier WM, et al. CSF biomarkers and medial temporal lobe atrophy predict dementia in mild cognitive impairment. Neurobiol Aging 2007;28:1070-1074.

15. American Psychiatric Association: Diagnostic and Statistical Manual of Mental Disorders,. 4th ed. Washington DC: American Psychiatric Association, 1994.

16. McKhann G, Drachman D, Folstein M, Katzman R, Price D, Stadlan EM. Clinical diagnosis of Alzheimer's disease: report of the NINCDS-ADRDA Work Group under the auspices of Department of Health and Human Services Task Force on Alzheimer's Disease. Neurology 1984;34:939-944.

17. Kolen M BR. Test Equating: Methods and Practices. New York: Springer-Verlag, 1995.

18. van de Pol LA, Verhey F, Frisoni GB, et al. White matter hyperintensities and medial temporal lobe atrophy in clinical subtypes of mild cognitive impairment: the DESCRIPA study. J Neurol Neurosurg Psychiatry 2009;80:1069-1074.

19. Wolz R, Aljabar P, Hajnal JV, Hammers A, Rueckert D. LEAP: learning embeddings for atlas propagation. Neuroimage 2010;49:1316-1325. 
20. Folstein MF, Folstein SE, McHugh PR. "Mini-mental state". A practical method for grading the cognitive state of patients for the clinician. J Psychiatr Res 1975;12:189-198.

21. Heagerty PJ, Lumley T, Pepe MS. Time-dependent ROC curves for censored s urvival data and a diagnostic marker. Biometrics 2000;56:337-344.

22. R Development Core Team. R: A language and environment for statistical computing. Vienna, 2010.

23. Vos S, van Rossum I, Burns L, et al. Test sequence of CSF and MRI biomarkers for prediction of AD in subjects with MCI. Neurobiol Aging 2012.

24. Visser PJ, Verhey F, Knol DL, et al. Prevalence and prognostic value of CSF markers of Alzheimer's disease pathology in patients with subjective cognitive impairment or mild cognitive impairment in the DESCRIPA study: a prospective cohort study. Lancet Neurol 2009;8:619-627.

25. Visser PJ, Kester A, Jolles J, Verhey F. Ten-year risk of dementia in subjects with mild cognitive impairment. Neurology 2006;67:1201-1207.

26. Kester MI, van der Vlies AE, Blankenstein MA, et al. CSF biomarkers predict rate of cognitive decline in Alzheimer disease. Neurology 2009;73:1353-1358.

27. Samgard K, Zetterberg H, Blennow K, Hansson O, Minthon L, Londos E. Cerebrospinal fluid total tau as a marker of Alzheimer's disease intensity. Int J Geriatr Psychiatry 2010;25:403-410.

28. Jack CR, Jr., Wiste HJ, Vemuri P, et al. Brain beta-amyloid measures and magnetic resonance imaging atrophy both predict time-to-progression from mild cognitive impairment to Alzheimer's disease. Brain 2010;133:3336-3348.

29. Schoonenboom NS, Reesink FE, Verwey NA, et al. Cerebrospinal fluid markers for differential dementia diagnosis in a large memory clinic cohort. Neurology 2012;78:47-54. 


\section{SUPPLEMENTAL DATA}

Supplemental Table e-1. Predictors for AD-type dementia

\begin{tabular}{lllll}
\hline & $\begin{array}{l}\text { Hazard ratio } \\
\text { uncorrected }\end{array}$ & p-value & $\begin{array}{l}\text { Hazard ratio } \\
\text { corrected }\end{array}$ & p-value \\
\hline CSF t-tau & $2.3(1.2-4.6)$ & 0.02 & $2.3(1.1-4.6)$ & 0.03 \\
CSF p-tau & $3.4(1.4-8.8)$ & 0.009 & $3.5(1.3-9.2)$ & 0.01 \\
Hippocampal atrophy & $1.7(0.9-3.4)$ & 0.1 & $2.5(1.1-5.6)$ & 0.02 \\
MMSE score & $1.8(1.1-3.0)$ & 0.02 & $2.3(1.3-4.0)$ & 0.003 \\
APOE genotype & $1.1(0.6-1.9)$ & 0.7 & $1.0(0.6-1.8)$ & 0.9 \\
Memory, learning & $1.1(0.6-2.1)$ & 0.8 & $1.1(0.6-2.2)$ & 0.7 \\
Memory, delayed recall & $2.2(1.1-4.3)$ & 0.03 & $2.1(1.0-4.3)$ & 0.05 \\
Verbal fluency & $1.7(1.0-2.9)$ & 0.06 & $1.5(0.8-2.9)$ & 0.2 \\
TMT part A & $1.1(0.6-2.0)$ & 0.7 & $1.1(0.6-2.0)$ & 0.8 \\
TMT part B & $1.4(0.7-2.5)$ & 0.3 & $1.5(0.8-2.9)$ & 0.3 \\
Visuoconstruction & $0.9(0.3-2.5)$ & 0.8 & $1.0(0.4-3.0)$ & 0.9 \\
\hline
\end{tabular}

${ }^{*}$ Analyses corrected for age, gender and educational level. All predictor variable were dichotomized scores. Hazard ratio's are provided with 95\% confidence intervals. Abnormal values were defined as: CSF t-tau $>375 \mathrm{pg} / \mathrm{ml}, \mathrm{CSF} \mathrm{p}-$ tau $>52 \mathrm{pg} / \mathrm{ml}$, hippocampal volume volume of $<5.39 \mathrm{~cm}^{3}$ (sum left and right), MMSE score $\leq 26$ at baseline or at least one APOE- $\varepsilon 4$ allele and a $z$-score of $\leq-1.5$ standard deviation on the neuropsychological tests. Abbreviations: $\mathrm{CSF}=$ cerebrospinal fluid, $\mathrm{A} \beta 1-42=$ beta amyloid1-42, $\mathrm{t}$-tau=total tau, $\mathrm{p}$-tau=tau phosphorylated at threonine 181, MMSE=Mini-Mental State Examination, APOE=apolipoprotein E, TMT=trail making test. 


\section{Supplemental Figure e-1. Selection of included subjects}

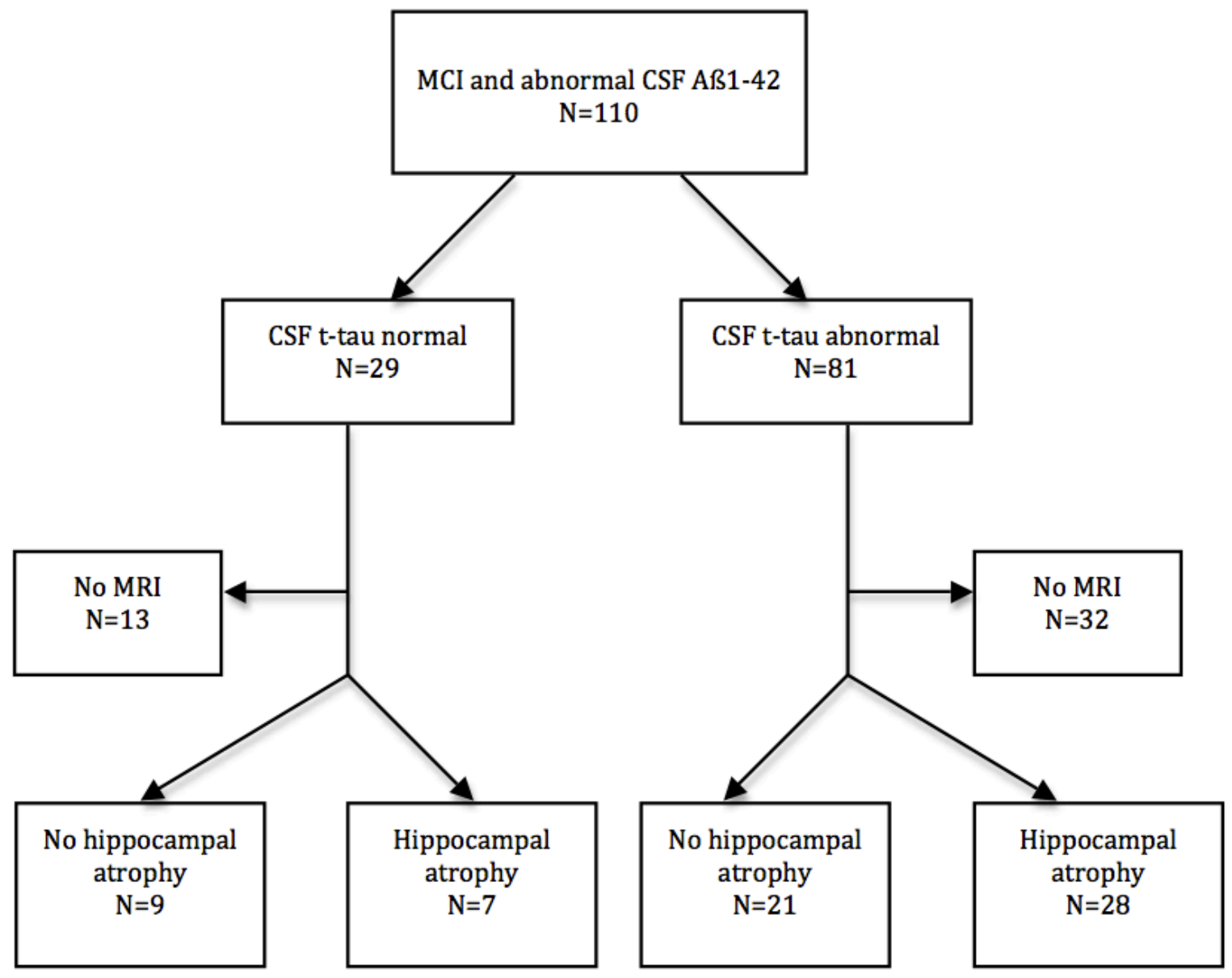

Flowchart of subjects included in the analyses depending on their biomarker status at baseline. $\mathrm{CSF}=$ cerebrospinal fluid, $\mathrm{t}$-tau=total tau, MMSE=Mini-Mental State Examination. 



\section{CHAPTER 6}

VARIABILITY OF CSF ALZHEIMER'S DISEASE BIOMARKERS:

IMPLICATIONS FOR CLINICAL PRACTICE

Stephanie J.B. Vos, Pieter Jelle Visser, Frans Verhey, Pauline Aalten, Inez Ramakers, Philip Scheltens, Marcel G.M. Olde Rikkert, Marcel M. Verbeek, Charlotte E. Teunissen 


\section{ABSTRACT}

\section{Background}

Cerebrospinal fluid (CSF) biomarkers are increasingly being used for diagnosis of Alzheimer's disease (AD).

\section{Objective}

We investigated the influence of CSF intralaboratory and interlaboratory variability on diagnostic CSF-based AD classification of subjects and identified causes of variation.

\section{Methods}

We measured CSF amyloid- $\beta(A \beta) 1-42$, total tau (t-tau), and phosphorylated tau ( $p$-tau) by INNOTEST enzyme-linked-immunosorbent assays (ELISA) in a memory clinic population $(n=126)$. Samples were measured twice in a single or two laboratories that served as reference labs for CSF analyses in the Netherlands. Predefined cut-offs were used to classify CSF biomarkers as normal or abnormal/AD pattern.

\section{Results}

CSF intralaboratory variability was higher for $A \beta 1-42$ than for t-tau and p-tau. Reanalysis led to a change in biomarker classification (normal vs. abnormal) of $26 \%$ of the subjects based on A $\beta 1-42,10 \%$ based on t-tau, and $29 \%$ based on p-tau. The changes in absolute biomarker concentrations were paralleled by a similar change in levels of internal control samples between different assay lots. CSF interlaboratory variability was higher for p-tau than for A $\beta 1-42$ and t-tau, and reanalysis led to a change in biomarker classification of $12 \%$ of the subjects based on $A \beta 1-42,1 \%$ based on t-tau, and $22 \%$ based on p-tau.

\section{Conclusions}

Intralaboratory and interlaboratory CSF variability frequently led to change in diagnostic CSF-based AD classification for A $\beta 1-42$ and p-tau. Lot-to-lot variation was a major cause of intralaboratory variability. This will have implications for the use of these biomarkers in clinical practice. 


\section{INTRODUCTION}

Amyloid- $\beta$ (A $\beta$ )1-42, total tau (t-tau), and phosphorylated tau ( $\mathrm{p}$-tau) proteins in cerebrospinal fluid (CSF) are well-established biomarkers for Alzheimer's disease (AD) ${ }^{[1-3]}$ and are increasingly being used for diagnosis in clinical practice. Previous studies reported considerable intra- or interlaboratory variability of CSF analyses, ${ }^{[4-7]}$ which may influence the diagnostic classification. In this study, we performed a large-scale CSF multicenter study and investigated the exact influence of intra- and interlaboratory variability on CSF-based AD classification of subjects.

We hypothesized that the change of diagnosis would be largest for classification of subjects based on CSF $A \beta 1-42$, as previous studies showed larger variability for CSF A $\beta 1-42$ than for t-tau and p-tau. ${ }^{[5-7]}$ It was also hypothesized that change of diagnosis would be lower for analyses performed in the same laboratory than for analyses performed in different laboratories because CSF intralaboratory variability has been reported to be lower than interlaboratory variability $(2.3-25 \%$ vs. $13-38 \%) .{ }^{[5-7]}$

We investigated intralaboratory and interlaboratory variability of CSF A $\beta 1-42$, t-tau, and p-tau analyses by INNOTEST enzyme-linked-immunosorbent assays (ELISA). Samples were measured twice in one or two laboratories that served as reference labs for AD CSF analyses in the Netherlands. We classified subjects based on validated cut-offs and examined how often CSF-based AD diagnosis changed after the second analysis.

\section{MATERIALS AND METHODS}

\section{CSF samples}

CSF samples were collected from subjects included in the Leiden Alzheimer Research Netherlands (LeARN) study. ${ }^{[8]}$ LeARN is a Dutch multicenter study performed in a memoryclinic setting that included subjects between October 2009 and May 2011 that had been newly referred for the assessment of cognitive complaints. Inclusion criteria were baseline diagnosis of subjective cognitive impairment ( $\mathrm{SCI}$ ), mild cognitive impairment (MCI) or dementia, Mini-Mental State Examination (MMSE) $\geq 20$, clinical dementia rating scale (CDR) of maximal 1. Exclusion criteria were somatic, psychiatric or neurological disorders that could have caused the cognitive impairment.

In 3 of the 4 participating centers (Amsterdam, Maastricht, and Nijmegen), 126 CSF samples were analyzed in twofold, the first time as part of clinical routine and secondly for the LeARN study (Supplemental Figure 1). For routine practice, samples were analyzed in Amsterdam and Nijmegen, which serve as national CSF AD biomarker centers. For the LeARN study, all samples were analyzed in a single batch in Amsterdam. The samples collected in Amsterdam ( $\mathrm{n}=50)$ were measured twice in the same lab and were used to assess intralaboratory variability. Samples collected in Nijmegen $(n=32)$ and Maastricht $(n=44)$ were measured twice in different laboratories, i.e. in Nijmegen for clinical routine and in Amsterdam for the LeARN study, and were used to study interlaboratory variability 
(total $\mathrm{n}=76$ ). Supplemental Table 1 provides baseline patient demographics. The medical ethics committee at each center approved the study. All subjects provided informed consent.

\section{CSF procedures and analyses}

CSF was obtained by lumbar puncture between the L3/L4 or L4/L5 intervertebral space, and collected and aliquoted into polypropylene tubes. Samples for clinical routine of Maastricht were transported the same day on room temperature or stored at $-20^{\circ} \mathrm{C}$ and transported on dry ice within one week to the Nijmegen laboratory for analysis. Samples for clinical routine analysis were stored at $-20^{\circ} \mathrm{C}$ for up to 4 weeks (Amsterdam cohort) or at $80^{\circ} \mathrm{C}$ for up to 2 weeks (Maastricht/Nijmegen cohort) before analysis. Research samples (i.e. samples of the LeARN study) were stored at $-80^{\circ} \mathrm{C}^{9}$ at each center and samples of Maastricht and Nijmegen were transported on dry ice to Amsterdam for analysis after up to 2.5 years. Both laboratories used the commercially available INNOTEST enzyme-linked immunosorbent assays (ELISAs; Innogenetics, Ghent, Belgium) to quantify CSF A $\beta 1-42$, CSF t-tau, and CSF p-tau, all performed by experienced laboratory technicians. For analysis of the research samples, the same lot number was used for all analyses, while for clinical routine analyses different lots were used in Nijmegen as well as in Amsterdam. Due to insufficient fluid material, CSF p-tau values were only available for 49 samples for intralaboratory analyses.

We also analyzed internal control samples from the Amsterdam lab to investigate the influence of lot-to-lot variation on measured CSF concentrations. One control sample had an AD typical profile and the other a normal CSF profile. The internal controls were aliquots for single use obtained by pooling surplus CSF. These aliquots were stored at $-80^{\circ} \mathrm{C}$ and all internal controls used in the current study were from the same batch of pools.

To study differences in biomarker classification as normal versus abnormal between CSF measurements, we dichotomized the CSF variables according to routinely used validated cut-offs of each lab. In Amsterdam, cut-offs were determined that could differentiate subjects with SCI from subjects with AD-type dementia with $85 \%$ sensitivity: CSF A $\beta 1-42 \leq 550 \mathrm{pg} / \mathrm{ml}$, t-tau $>375 \mathrm{pg} / \mathrm{ml}$, and p-tau $>52 \mathrm{pg} / \mathrm{ml} .{ }^{[10]}$ In Nijmegen, cut-offs were determined that could differentiate cognitively normal controls from subjects with ADtype dementia with a specificity of 95\%: CSF A $\beta 1-42<500 \mathrm{pg} / \mathrm{ml}$, t-tau $>350 \mathrm{pg} / \mathrm{ml}$, and ptau $>85 \mathrm{pg} / \mathrm{ml}$.[11] Given that a different approach was used to define cut-offs in Amsterdam and Nijmegen, we performed interlaboratory analyses with the same cut-offs (Amsterdam cut-offs) as well as with lab-specific cut-offs (Amsterdam and Nijmegen cut-offs).

\section{Statistical analyses}

Statistical analyses were done with SPSS version 19.0 (Chicago, IL, USA) and GraphPad Prism 5, with significance set at $\mathrm{p}<0.05$. Intralaboratory and interlaboratory coefficients of variation (CV) were calculated as the standard deviation (SD) divided by the mean of the measurements of each sample for each biomarker. Subsequently, a mean CV was calculated. 
We performed paired t-tests to investigate the intralaboratory and interlaboratory variability between CSF analyses of CSF A 1-42, t-tau, and p-tau. In addition, we calculated Pearson correlations $r$ and Intraclass Correlation Coefficients (ICC). An ICC score ranges from 0 to 1 , representing virtually no (0.00-0.10), a slight (0.11-0.40), fair (0.41-0.60), moderate (0.61-0.80), or substantial (0.81-1.00) level of agreement between the analyses.[12] Confidence intervals were calculated for both the Pearson correlations and ICC scores. We made Bland-Altman plots to visualize the agreement between the CSF analyses ${ }^{[13]}$ and calculated the percentage of subjects with change of AD marker classification after the second analysis using routine CSF cut-offs of each laboratory.

\section{RESULTS}

\section{Intralaboratory variability}

CSF A 1-42 and t-tau levels were higher and p-tau levels were lower after reanalysis in the same laboratory $(p<0.05$ for all analysis, Figure $1 \mathrm{~A}$, Supplemental Figure $2 \mathrm{~A})$. The mean intralaboratory $\mathrm{CV}$ was $14.4 \%$ for $\mathrm{A} \beta 1-42,8.5 \%$ for $\mathrm{t}$-tau, and $12.6 \%$ for p-tau. For CSF $\mathrm{A} \beta 1$ 42 , the correlation (0.85) and ICC (0.76) were moderate and lower than that of CSF t-tau $(r=0.98$ and ICC $=0.97)$ and $p$-tau $(r=0.95$ and ICC $=0.90$; Table 1, Figure $2 \mathrm{~A})$. Internal quality control samples that were analyzed at each measurement also showed higher A $\beta 1-42$ levels, slightly higher t-tau levels, and lower p-tau levels in the second measurement compared to the first measurement (Figure 3).

\section{Interlaboratory variability}

CSF A 1-42 levels were lower, $t$-tau levels higher, and $p$-tau levels lower after reanalysis in the second laboratory ( $\mathrm{p}<0.05$ for all analysis, Figure 1B, Supplemental Figure 2B). The interlaboratory $\mathrm{CV}$ was $7.3 \%$ for $\mathrm{A} \beta 1-42,6.7 \%$ for $\mathrm{t}$-tau, and $27.6 \%$ for p-tau. For CSF p-tau, the correlation (0.94) and the ICC (0.73) were high to moderate and lower than that of CSF Aß1-42 ( $r=0.94$ and ICC=0.92) and t-tau ( $r=0.98$ and ICC=0.98; Table 1, Figure 2B).

Table 1. Agreement between CSF analyses

\begin{tabular}{lllllll}
\hline & \multicolumn{2}{l}{ Intralaboratory analyses } & & \multicolumn{2}{l}{ Interlaboratory analyses } \\
\cline { 2 - 3 } \cline { 6 - 7 } & \multicolumn{2}{l}{ Correlation* $^{*}$} & ICC & & Correlation & ICC $^{*}$ \\
\hline CSF A $\beta 1-42$ & $0.85(0.74-0.91)$ & $0.76(0.43-0.89)$ & & $0.94(0.91-0.96)$ & $0.92(0.85-0.96)$ \\
CSF t-tau & $0.98(0.97-0.99)$ & $0.97(0.92-0.99)$ & & $0.98(0.96-0.99)$ & $0.98(0.96-0.99)$ \\
CSF p-tau & $0.95(0.92-0.97)$ & $0.90(0.53-0.97)$ & & $0.94(0.90-0.96)$ & $0.73(0.07-0.92)$ \\
\hline
\end{tabular}

Results are Pearson correlation and ICC (95\% CI) for intra- and interlaboratory CSF analyses. ICC=Intraclass coefficients, ratio $=A \beta 1-42 / \mathrm{t}$-tau, $\mathrm{CSF}=$ cerebrospinal fluid, $\mathrm{A} \beta=$ amyloid beta, $\mathrm{t}$-tau=total tau, $\mathrm{p}$-tau=phosphorylated tau. *All $\mathrm{p}<0.001$. 

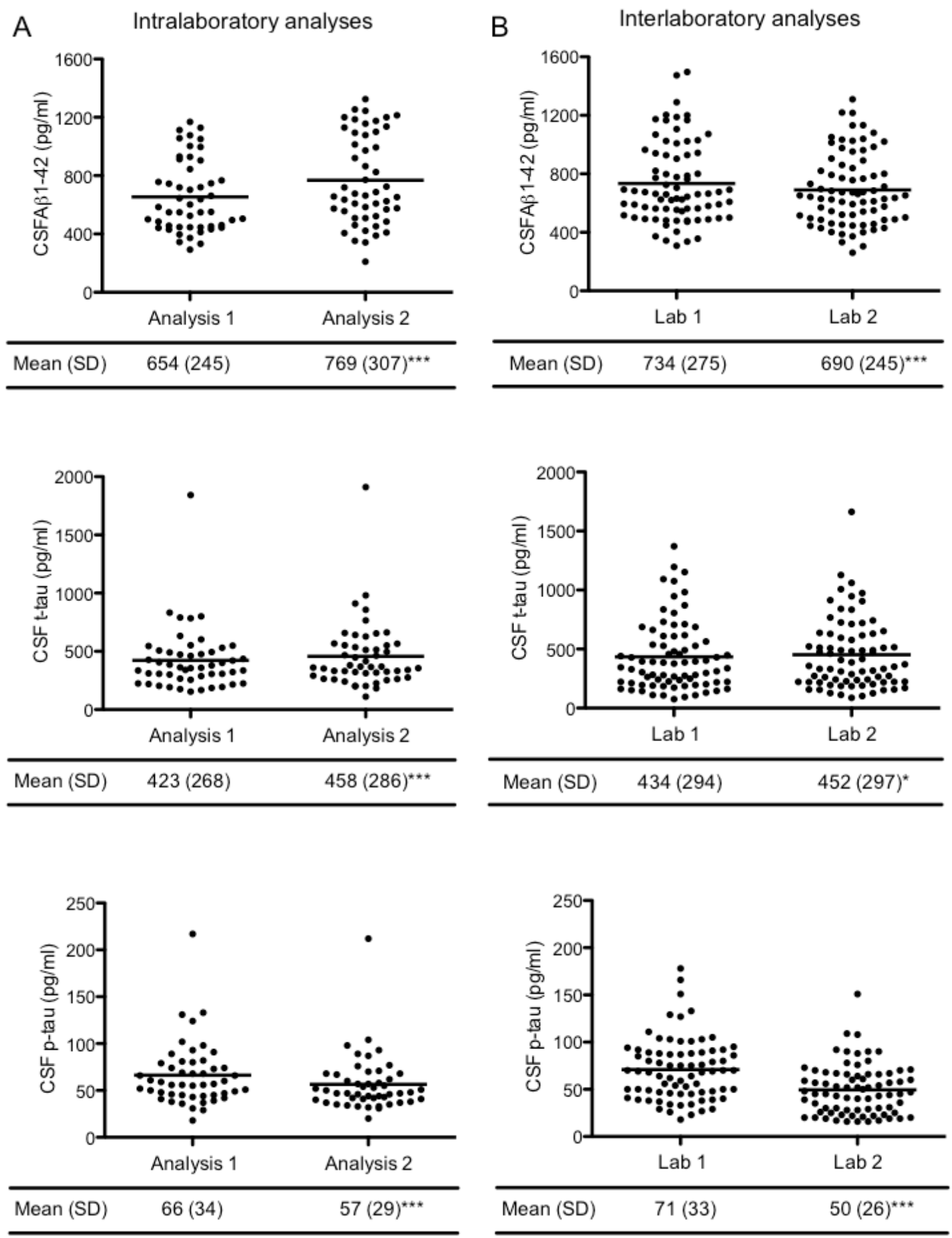

Figure 1. CSF levels by analysis and marker

Results are frequencies and mean (SD) for each CSF marker on the left (A) for CSF intralaboratory analyses and on the right (B) for CSF interlaboratory analyses. The solid line represents the mean CSF levels. Analysis 1 is routine practice and analysis 2 is performed as part of the LeARN study. CSF=cerebrospinal fluid, $A \beta=$ amyloid beta, $t$-tau=total tau, $p$ tau=phosphorylated tau. ${ }^{* *} \mathrm{P}<0.001,{ }^{*} \mathrm{p}<0.05$ compared to CSF analysis 1 or lab 1. 
A Intralaboratory analyses

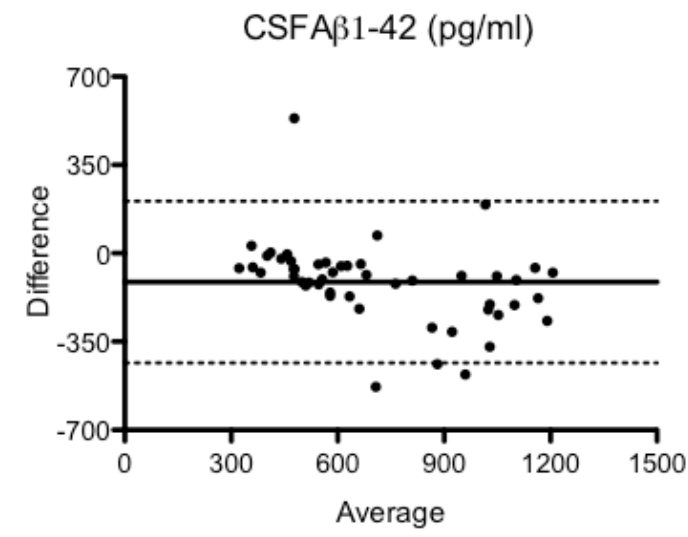

CSF t-tau $(\mathrm{pg} / \mathrm{ml})$

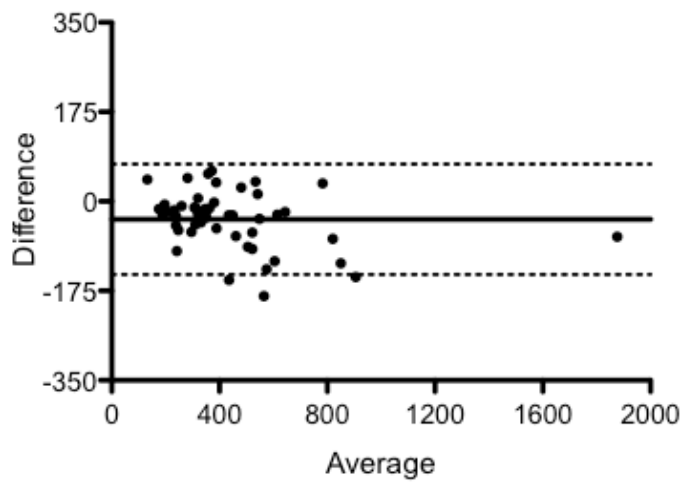

CSF p-tau $(p g / m l)$

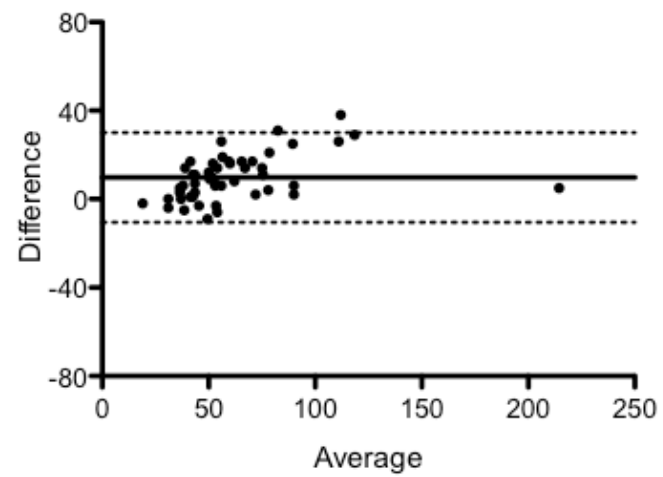

B Interlaboratory analyses

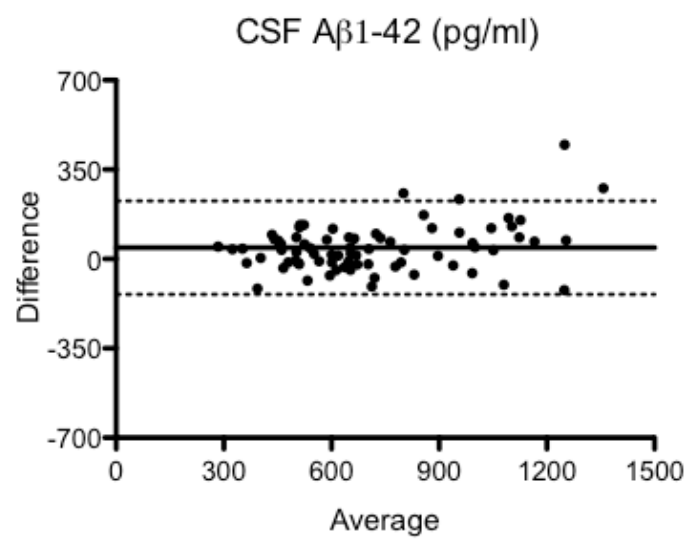

CSF t-tau $(\mathrm{pg} / \mathrm{ml})$

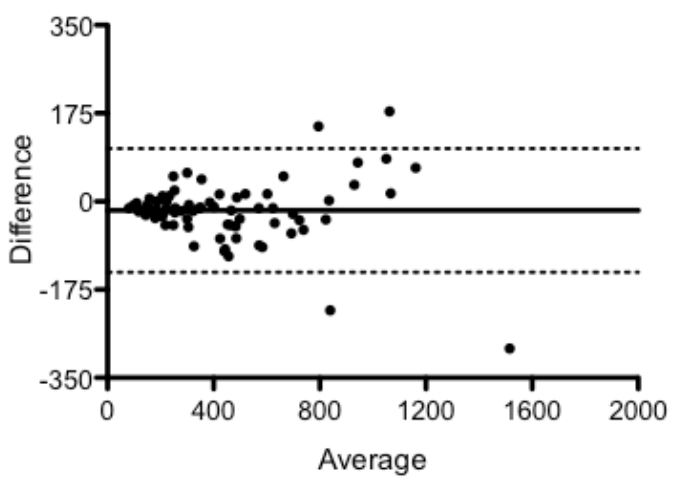

CSF p-tau $(p g / m l)$

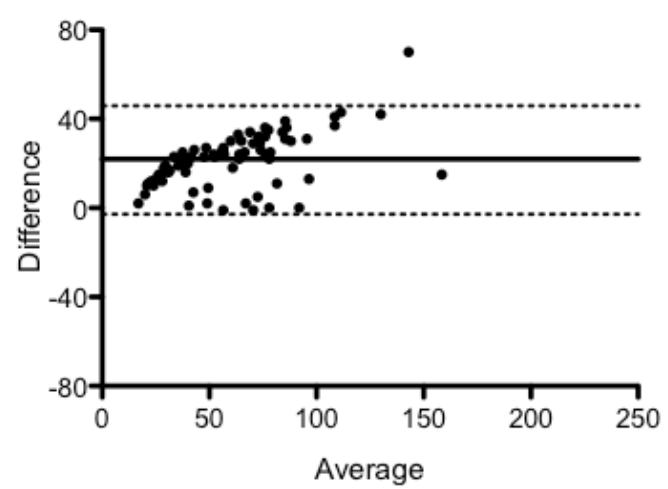

Figure 2. Bland-Altman plots of variability between CSF analyses

The average of CSF analysis 1 and 2 is plotted against the difference between both analyses, on the left (A) for CSF intralaboratory analyses and on the right (B) for CSF interlaboratory analyses. The solid line represents the mean and the dotted lines the upper and lower $1.95 \mathrm{SD}$. CSF=cerebrospinal fluid, $A \beta=a$ myloid beta, $t$-tau=total tau, $p$ tau=phosphorylated tau. 

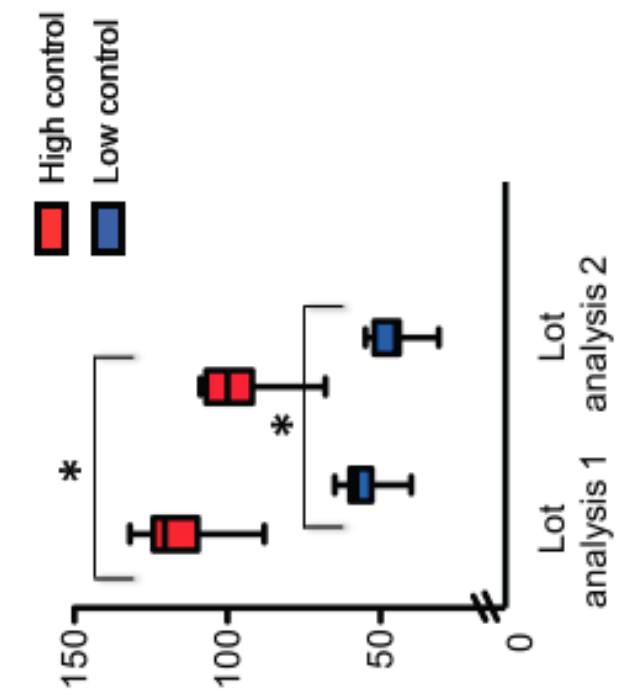

(ךm/6d) nę-d 」Sว

$\cup$

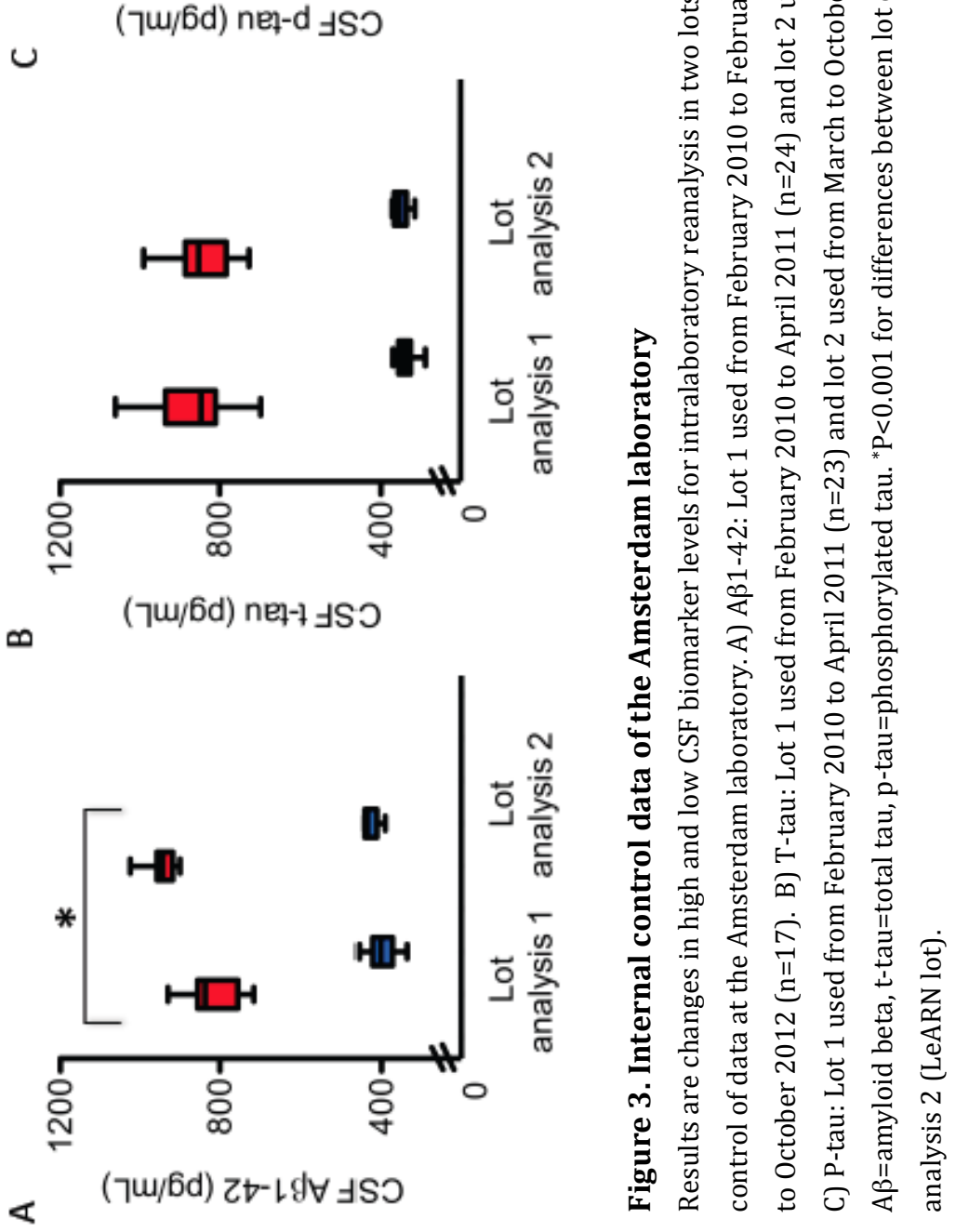




\section{Change in AD classification}

We investigated how reanalysis changed the CSF AD classification based on individual CSF markers and based on the combination of CSF markers with an AD profile being defined as abnormal A 1 1-42 and abnormal t-tau or p-tau. Using predefined cutoffs, repeated CSF analyses in the same laboratory led to a change in biomarker classification (normal vs. abnormal) of $26 \%$ of subjects based on A $\beta 1-42,10 \%$ based on t-tau, $29 \%$ based on p-tau, and $16 \%$ based on the AD profile (Table 2). Repeated CSF analyses in different laboratories using the cut-offs from the Amsterdam lab led to a change in biomarker classification of $12 \%$ of subjects based on CSF A $\beta 1-42,1 \%$ based on t-tau, $22 \%$ based on p-tau, and $14 \%$ based on the AD profile (Table 2). When we applied lab-specific cut-offs to define an abnormal score, the repeated CSF analyses in different laboratories led to a change in biomarker classification of $17 \%$ of subjects based on CSF A $\beta 1-42,1 \%$ based on t-tau, $12 \%$ based on p-tau, and $12 \%$ based on the AD profile (Table 2). Figure 4 shows the change in CSF levels for each biomarker for intra- and interlaboratory analyses of subjects in whom reanalysis led to a different biomarker classification as normal vs. abnormal when Amsterdam cut-offs were applied. Most of the subjects with a change in AD classification after reanalysis in the same laboratory as well as in a different laboratory had CSF biomarker values around the cut-off points. 


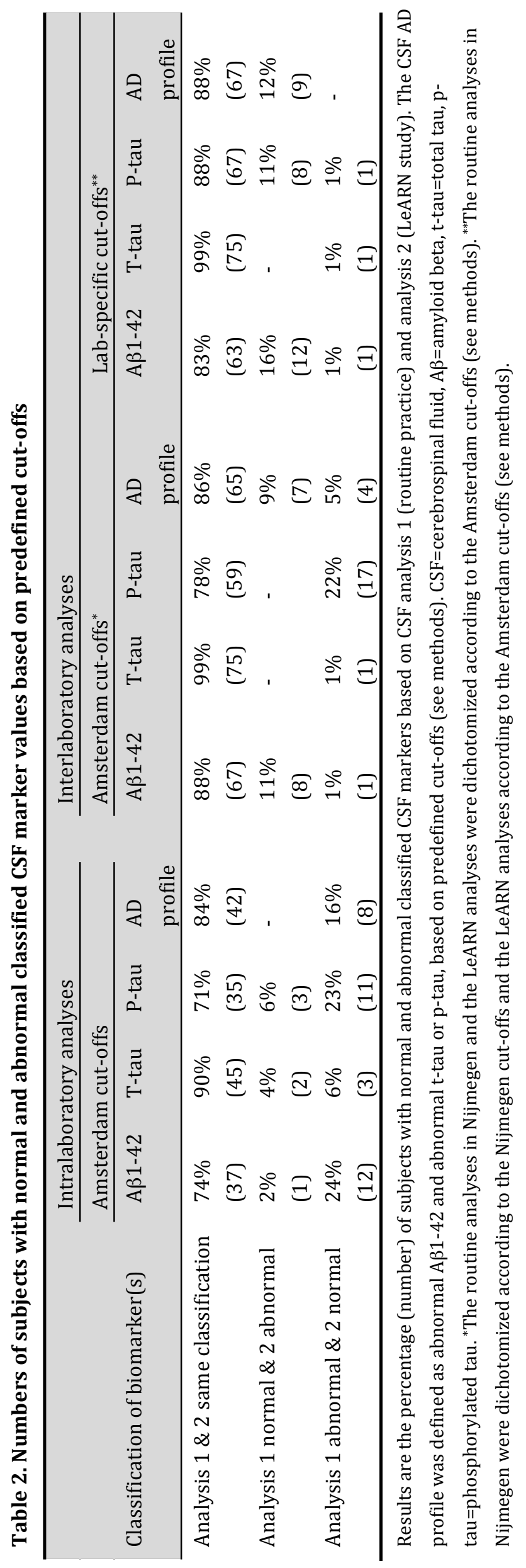



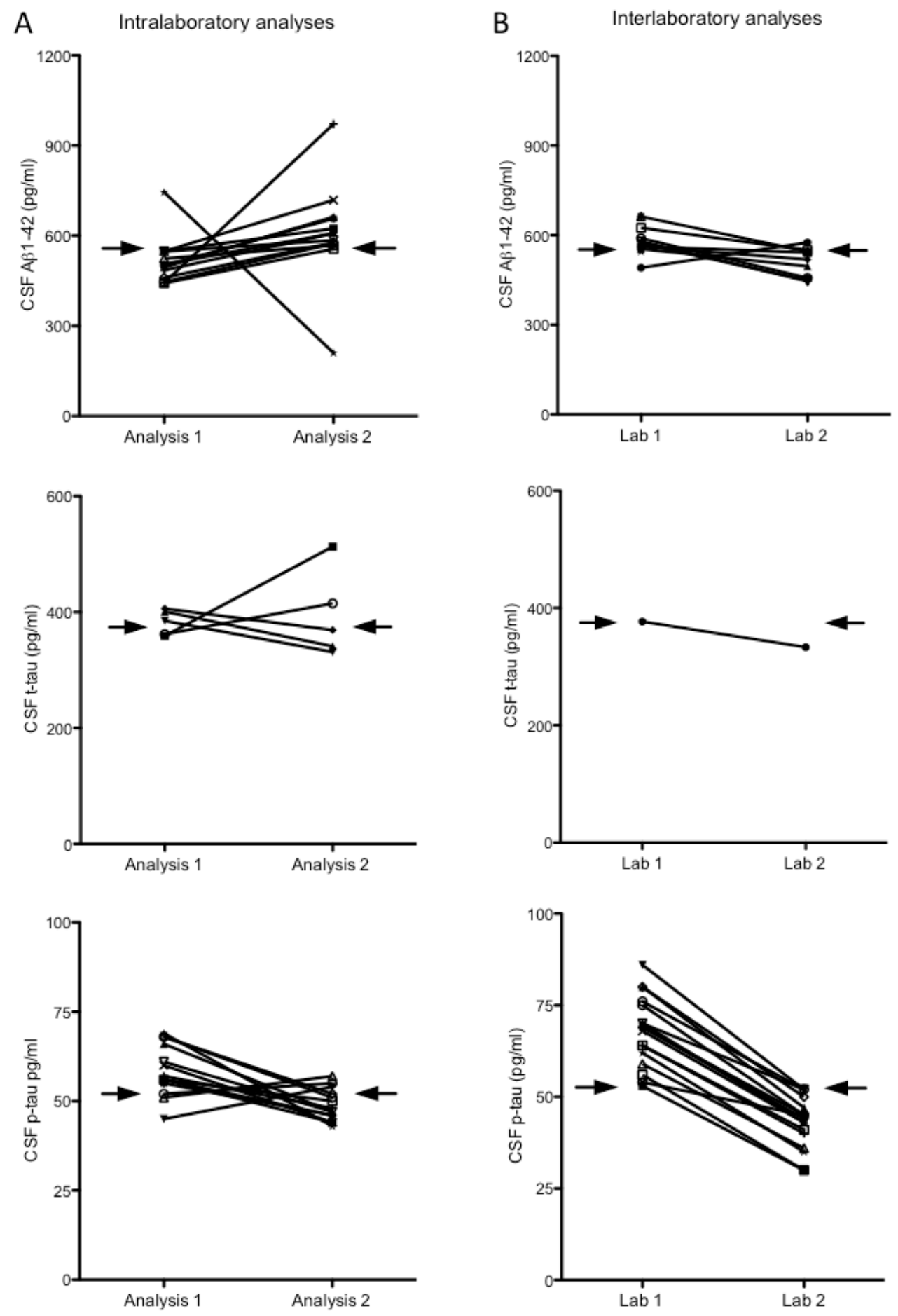

Figure 4. Change in CSF marker classification according to cut-offs

Results are CSF levels only for subjects in which reanalysis let to a different biomarker classification using the cut-offs from Amsterdam to define abnormal CSF values. On the left (A) are changes in biomarker levels for intralaboratory reanalysis and on the right (B) for interlaboratory reanalysis. The arrow represents the applied Amsterdam CSF cutoff. Analysis 1 is routine practice and analysis 2 is performed as part of the LeARN study. CSF=cerebrospinal fluid, $\mathrm{A} \beta=$ amyloid beta, $\mathrm{t}$-tau=total tau, $\mathrm{p}$-tau=phosphorylated tau . 


\section{DISCUSSION}

We observed clear variability in CSF AD biomarker levels between repeated analyses in the same laboratory as well as between two different laboratories. Our study is the first to show that this variability frequently led to a change in CSF-based AD diagnosis when predefined cut-offs for abnormal CSF values were applied.

CSF intralaboratory variability (based on CV) was largest for A $\beta 1-42$, consistent with previous studies. ${ }^{[5-7]}$ Change in AD-like scores after repeated analyses in the same laboratory was, however, highest for CSF p-tau (29\%) followed by A $\beta 1-42$ (26\%) and t-tau $(10 \%)$. This large change for p-tau is likely due to a smaller range of values of p-tau and values being closer to the cut-off compared to other markers, such that a small change in observed concentration more easily leads to a different classification.

CSF interlaboratory variability (based on CV) was largest for p-tau, unlike findings from previous studies, which found that interlaboratory variability was largest for A $\beta 1-42 .{ }^{[5-}$ 7] Change in AD-like scores after repeated analyses in different laboratories was also higher for CSF p-tau (22\%) than for A $\beta 1-42(12 \%)$ and $t$-tau $(1 \%)$ when the same cut-offs were used for each site. A rather unexpected finding was that the interlaboratory variability of A 1-42 was smaller than the intralaboratory variability.

A major finding was that CSF analytical variability frequently led to a change in diagnostic CSF-based AD classification. Importantly, also a diagnosis of a CSF AD profile based on A $\beta 1-42$ and t-tau or p-tau changed in 12-16\% of the cases. As most subjects whose $\mathrm{AD}$ classification changed after reanalysis had values around the cut-off points, it could be helpful to use a range around a cut-off point rather than a fixed cut-off point.

The intra- and interlaboratory variability in CSF results can result from differences in preanalytical and analytical procedures, and lot-to-lot variation of analytical kits. ${ }^{[9,15]}$ The intralaboratory variability is likely due to lot-to-lot variation as other procedures remained essentially the same. Additional support for this comes from the observation that internal control values showed a striking lot-to-lot variation of up to $20 \%$. The change in internal control values was of the same order of magnitude and same direction of change as the change in CSF scores from the patient samples. Another possible explanation for the intralaboratory variability is variability in freezing conditions and storage time. However, previous studies showed that these factors have a minor impact on variability of CSF values. ${ }^{[16,17]}$

Interlaboratory variability may also be caused by lot-to-lot variation. Indeed, lots used in each lab showed only minor overlap (data not shown). Differences in analytical procedures may also have contributed to the interlaboratory variability. However, both laboratories used similar protocols, were both trained in a hands-on workshop, ${ }^{[9]}$ and were similarly experienced.

Another source of variability between laboratories, which may influence CSF-based AD classification, is the difference in cut-offs used. We, therefore, tested interlaboratory variability for CSF-based AD classification both with the same CSF cut-offs as well as labspecific cut-offs. While lab-specific cut-offs for A $\beta 1-42$ and t-tau did not differ much, there 
was a large difference for p-tau (85 pg/ml in Nijmegen vs. $52 \mathrm{pg} / \mathrm{ml}$ in Amsterdam). This was also reflected in the interlaboratory variability in CSF-based AD classification. Using the same cut-off, abnormal p-tau was more common when samples were analyzed in Nijmegen than in Amsterdam. Change in classification was mostly due to subjects with scores around the cut-off (Figure 4B). Using the lab-specific cut-off, however, abnormal p-tau was more common when samples were analyzed in Amsterdam than in Nijmegen. Here change in classification was mainly due to differences in cut-offs. This clearly indicates that labspecific cut-offs may also influence comparability between laboratories.

Our study has several limitations. The cut-offs that were used may have influenced our findings on change in CSF-based AD diagnosis. However, as no universal CSF cut-offs are available, we applied routinely used validated cut-offs. One of the major strengths of this study was the large number of CSF samples used to study interlaboratory variability, as most previous studies were based only on a few samples. ${ }^{[5-7]}$ In addition, our study design allows generalization to other CSF centers that analyze CSF AD biomarkers using ELISA, as it reflects CSF procedures in general clinical practice.

Together, our findings suggest that variability in CSF analyses is common between and within laboratories, in particular for A $\beta 1-42$ and p-tau. A substantial part of this variability seems to be explained by lot-to-lot variation of analytical kits. The variability has a large impact on CSF-based AD diagnosis or treatment decisions in clinical settings, suggesting that we should be careful when interpreting CSF findings and always interpret them within a clinical context ${ }^{17}$ and with reference to internal standards. For the moment this rightly restricts the indication for CSF biomarker testing in diagnostic guidelines as complimentary and non-obligatory. ${ }^{[19,20]}$ The recent consensus on standardization of preanalytical aspects of CSF analyses, ${ }^{[15,17]}$ as well as the ongoing worldwide quality control study[6] and standardization projects (www.neurodegenerationresearch.eu) will help to move towards a standardized and harmonized implementation of CSF markers in clinical routine. 


\section{REFERENCES}

1. Blennow K, Hampel H (2003) CSF markers for incipient Alzheimer's disease. Lancet Neurol 2, 605613.

2. Fagan AM, Holtzman DM (2010) Cerebrospinal fluid biomarkers of Alzheimer's disease. Biomark Med 4, 51-63.

3. Vos SJB, van Rossum IA, Verhey F, Knol DL, Soininen H, Wahlund L-O, Hampel H, Tsolaki M, Minthon L, Frisoni GB, Froelich L, Nobili F, van der Flier W, Blennow K, Wolz R, Scheltens P, Visser PJ (2013) Prediction of Alzheimer's disease in subjects with amnestic and non-amnestic MCI. Neurology 80, 1124-1132.

4. Dumurgier J, Vercruysse O, Paquet C, Bombois S, Chaulet C, Laplanche JL, Peoc'h K, Schraen S, Pasquier F, Touchon J, Hugon J, Lehmann S, Gabelle A (2012) Intersite variability of CSF Alzheimer's disease biomarkers in clinical setting. Alzheimers Dement. DOI: 10.1016/j.jalz.2012.06.006

5. Lewczuk P, Beck G, Ganslandt O, Esselmann H, Deisenhammer F, Regeniter A, Petereit HF, Tumani H, Gerritzen A, Oschmann P, Schröder J, Schönknecht P, Zimmermann K, Hampel H, Bürger K, Otto M, Haustein S, Herzog K, Dannenberg R, Wurster U, Bibl M, Maler JM, Reubach U, Kornhuber J, Wiltfang J (2006) International quality control survey of neurochemical dementia diagnostics. Neurosci Lett 409, 1-4.

6. Mattsson N, Andreasson U, Persson S, Arai H, Batish SD, Bernardini S, Bocchio-Chiavetto L, Blankenstein MA, Carrillo MC, Chalbot S, Coart E, Chiasserini D, Cutler N, Dahlfors G, Duller S, Fagan AM, Forlenza O, Frisoni GB, Galasko D, Galimberti D, Hampel H, Handberg A, Heneka MT, Herskovits AZ, Herukka SK, Holtzman DM, Humpel C, Hyman BT, Iqbal K, Jucker M, Kaeser SA, Kaiser E, Kapaki E, Kidd D, Klivenyi P, Knudsen CS, Kummer MP, Lui J, Lladó A, Lewczuk P, Li QX, Martins R, Masters C, McAuliffe J, Mercken M, Moghekar A, Molinuevo JL, Montine TJ, Nowatzke W, O'Brien R, Otto M, Paraskevas GP, Parnetti L, Petersen RC, Prvulovic D, de Reus HP, Rissman RA, Scarpini E, Stefani A, Soininen H, Schröder J, Shaw LM, Skinningsrud A, Skrogstad B, Spreer A, Talib L, Teunissen C, Trojanowski JQ, Tumani H, Umek RM, Van Broeck B, Vanderstichele H, Vecsei L, Verbeek MM, Windisch M, Zhang J, Zetterberg H, Blennow K (2011) The Alzheimer's Association external quality control program for cerebrospinal fluid biomarkers. Alzheimers Dement 7, 386-395.

7. Verwey NA, van der Flier WM, Blennow K, Clark C, Sokolow S, De Deyn PP, Galasko D, Hampel H, Hartmann T, Kapaki E, Lannfelt L, Mehta PD, Parnetti L, Petzold A, Pirttila T, Saleh L, Skinningsrud A, Swieten JC, Verbeek MM, Wiltfang J, Younkin S, Scheltens P, Blankenstein MA (2009) A worldwide multicentre comparison of assays for cerebrospinal fluid biomarkers in Alzheimer's disease. Ann Clin Biochem 46, 235-240.

8. Handels RL, Aalten P, Wolfs CA, Olde Rikkert M, Scheltens P, Visser PJ, Joore MA, Severens JL, Verhey FR (2012) Diagnostic and economic evaluation of new biomarkers for Alzheimer's disease: the research protocol of a prospective cohort study. BMC Neurol 12, 72.

9. Teunissen CE, Verwey NA, Kester MI, van Uffelen K, Blankenstein MA (2010) Standardization of Assay Procedures for Analysis of the CSF Biomarkers Amyloid beta((1-42)), Tau, and Phosphorylated Tau in Alzheimer's Disease: Report of an International Workshop. Int J Alzheimers Dis. doi: 10.4061/2010/635053.

10. Mulder C, Verwey NA, van der Flier WM, Bouwman FH, Kok A, van Elk EJ, Scheltens P, Blankenstein MA (2010) Amyloid-beta(1-42), total tau, and phosphorylated tau as cerebrospinal fluid biomarkers for the diagnosis of Alzheimer disease. Clin Chem 56, 248-253.

11. de Jong D, Kremer BP, Olde Rikkert MG, Verbeek MM (2007) Current state and future directions of neurochemical biomarkers for Alzheimer's disease. Clin Chem Lab Med 45, 1421-1434. 
12. Shrout PE (1998) Measurement reliability and agreement in psychiatry. In Stat Methods Med Res, 7, pp. 301-317.

13. Bland JM, Altman DG (1986) Statistical methods for assessing agreement between two methods of clinical measurement. Lancet 1, 307-310.

14. Hulstaert F, Blennow K, Ivanoiu A, Schoonderwaldt HC, Riemenschneider M, De Deyn PP, Bancher C, Cras P, Wiltfang J, Mehta PD, Iqbal K, Pottel H, Vanmechelen E, Vanderstichele H. (1999) Improved discrimination of AD patients using [beta]-amyloid(1-42) and tau levels in CSF. Neurology 52, 15551562.

15. del Campo M, Mollenhauer B, Bertolotto A, Engelborghs S, Hampel H, Simonsen AH, Kapaki E, Kruse N, Le Bastard N, Lehmann S, Molinuevo JL, Parnetti L, Perret-Liaudet A, Sáez-Valero J, Saka E, Urbani A, Vanmechelen E, Verbeek M, Visser PJ, Teunissen C (2012) Recommendations to standardize preanalytical confounding factors in Alzheimer's and Parkinson's disease cerebrospinal fluid biomarkers: an update. Biomark Med 6, 419-430.

16. Schoonenboom NS, Mulder C, Vanderstichele H, Van Elk EJ, Kok A, Van Kamp GJ, Scheltens P, Blankenstein MA (2005) Effects of processing and storage conditions on amyloid beta (1-42) and tau concentrations in cerebrospinal fluid: implications for use in clinical practice. Clin Chem 51, 189-195.

17. Vanderstichele H, Bibl M, Engelborghs S, Le Bastard N, Lewczuk P, Molinuevo JL, Parnetti L, PerretLiaudet A, Shaw LM, Teunissen C, Wouters D, Blennow K (2012) Standardization of preanalytical aspects of cerebrospinal fluid biomarker testing for Alzheimer's disease diagnosis: a consensus paper from the Alzheimer's Biomarkers Standardization Initiative. Alzheimers Dement 8, 65-73.

18. Spies PE, Claassen JA, Peer PG, Blankenstein MA, Teunissen CE, Scheltens P, van der Flier WM, Olde Rikkert MG, Verbeek MM (2012) A prediction model to calculate probability of Alzheimer's disease using cerebrospinal fluid biomarkers. Alzheimers Dement; doi: 10.1016/j.jalz.2012.01.010.

19. Hort J, O'Brien JT, Gainotti G, Pirttila T, Popescu BO, Rektorova I, Sorbi S, Scheltens P; EFNS Scientist Panel on Dementia (2010). EFNS guidelines for the diagnosis and management of Alzheimer's disease. Eur J Neurol 17, 1236-1248.

20. Jack CR, Albert MS, Knopman DS, McKhann GM, Sperling RA, Carrillo MC, Thies B, Phelps CH (2011) Introduction to the recommendations from the National Institute on Aging-Alzheimer's Association workgroups on diagnostic guidelines for Alzheimer's disease. Alzheimers Dement 7, 257-262. 


\section{SUPPLEMENTAL DATA}

Supplemental Table 1. Baseline patient demographics

\begin{tabular}{llll}
\hline & $\begin{array}{l}\text { Total sample } \\
(\mathrm{n}=126)\end{array}$ & $\begin{array}{l}\text { Amsterdam cohort } \\
(\mathrm{n}=50)\end{array}$ & $\begin{array}{l}\text { Maastricht \& Nijmegen } \\
\text { cohort (n=76) }\end{array}$ \\
\hline Age & $67.0(9.2)$ & $65.0(7.7)$ & $68.3(9.8)$ \\
Female, $\mathrm{n}$ & $36(29 \%)$ & $12(24 \%)$ & $24(32 \%)$ \\
MMSE & $26.1(2.9)$ & $25.2(3.0)$ & $26.8(2.7)$ \\
CDR & $0.6(0.3)$ & $0.7(0.4)$ & $0.5(0.3)$ \\
Clinical diagnosis, $\mathrm{n}$ & & & $32(42 \%)$ \\
SCI & $41(33 \%)$ & $9(18 \%)$ & $25(33 \%)$ \\
MCI & $33(26 \%)$ & $8(16 \%)$ & $15(20 \%)$ \\
AD-type dementia & $32(25 \%)$ & $17(34 \%)$ & $4(5 \%)$ \\
Other dementia & $20(16 \%)$ & $16(32 \%)$ & \\
\hline
\end{tabular}

Results are mean (SD) or number (\%), presented for the total sample and separately for the sample for intralaboratory analyses (Amsterdam cohort) and the sample for interlaboratory analyses (Maastricht \& Nijmegen cohort). MMSE=Mini-Mental State Examination, $C D R=C l i n i c a l$ dementia rating scale, SCI=subjective cognitive impairment, $\mathrm{MCI}=$ mild cognitive impairment, $\mathrm{AD}=$ Alzheimer's disease.

\begin{tabular}{|l|l|l|}
\hline $\begin{array}{l}\text { Amsterdam } \\
\text { (samples Amsterdam, } \mathrm{n}=49-50)\end{array}$ & Intra-assay variation & $\begin{array}{l}\text { Amsterdam } \\
\text { (all samples) }\end{array}$ \\
$\begin{array}{l}\text { Nijmegen } \\
\text { (samples Maastricht and Nijmegen, } \mathrm{n}=76)\end{array}$ & Interassay variation \\
\cline { 1 - 2 }
\end{tabular}

Analysis 1: routine

Analysis 2: LeARN

\section{Supplemental Figure 1. CSF analyses by laboratory}

Laboratories that performed CSF analyses for clinical routine are presented on the left (analysis 1); the laboratory that performed CSF analyses for the LeARN study is presented on the right (analysis 2). 
A
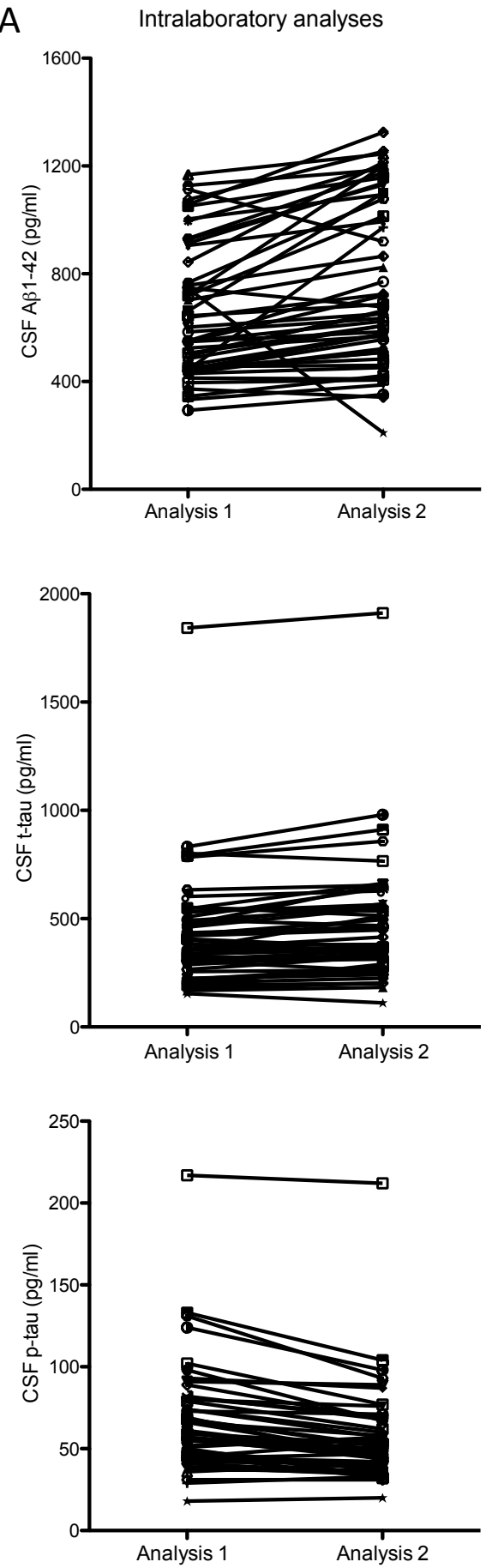

B Interlaboratory analyses
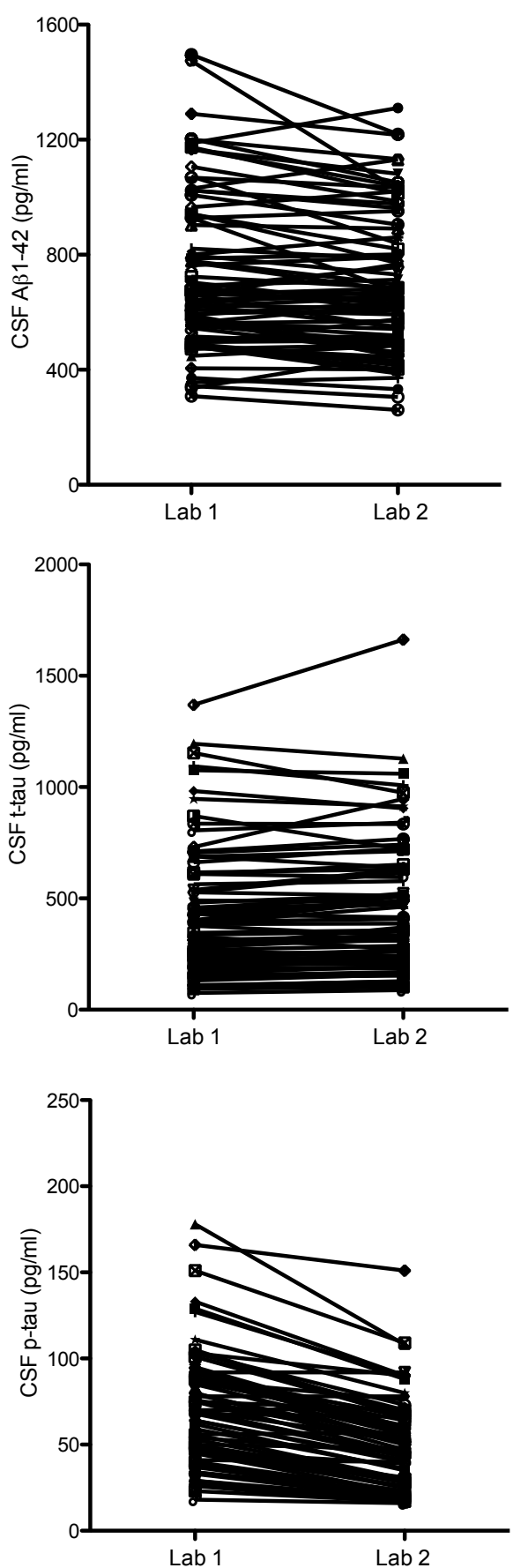

\section{Supplemental Figure 2. Change in CSF levels after reanalysis}

Results on the left (A) are changes in CSF biomarker levels for intralaboratory reanalysis and on the right (B) for interlaboratory reanalysis. Analysis 1 is routine practice and analysis 2 is performed as part of the LeARN study. $\mathrm{CSF}=$ cerebrospinal fluid, $\mathrm{A} \beta=$ amyloid beta, $\mathrm{t}$-tau=total tau, $\mathrm{p}$-tau=phosphorylated tau. 



\section{PART II}

\section{DIAGNOSTIC CRITERIA FOR}

\section{PREDEMENTIA}

ALZHEIMER'S DISEASE 



\section{CHAPTER 7}

COMPARISON OF INTERNATIONAL WORKING GROUP CRITERIA AND NATIONAL INSTITUTE ON AGING - ALZHEIMER'S ASSOCIATION CRITERIA FOR ALZHEIMER'S DISEASE

Alzheimer's \& Dementia 2012;8:560-563

Pieter Jelle Visser, Stephanie Vos, Ineke van Rossum, Philip Scheltens 


\section{ABSTRACT}

Two sets of research criteria for Alzheimer's disease (AD) are now available: those published by an International Working Group (IWG) in 2007 and the recommendations published by the National Institute of Aging and the Alzheimer Association (NIA-AA) in 2011. They provide both guidelines for the diagnosis of asymptomatic and symptomatic AD. The co-existence of two sets of criteria for the same disorder raises the question as to which set of criteria should be preferred. A comparison of the criteria revealed differences in approach, terminology and use of cognitive markers and biomarkers. Most persons who meet the IWG criteria will also meet the NIA-AA criteria and vice versa. However, the NIAAA criteria allow for a subclassification of persons based on biomarker results within each diagnostic category. Further research is needed to validate the criteria. Modifications are likely to be made before the criteria can be used in daily practice. 


\section{INTRODUCTION}

Two sets of research criteria for Alzheimer's disease (AD) are now available: those published by an International Working Group (IWG) in 2007 and those published by the National Institute of Aging and the Alzheimer Association (NIA-AA) in this Journal in 2011 [1-4]. The co-existence of two sets of criteria for the same disorder raises the question as to which set of criteria should be used. In this commentary, we will compare the two sets of criteria, highlight their strengths and limitations, and discuss future perspectives.

\section{APPROACH AND TERMINOLOGY}

$\mathrm{AD}$ can be subdivided in three stages based on the presence of AD biomarkers and cognitive impairments: a prepathology stage (biomarkers normal, no cognitive impairments) an asymptomatic stage (biomarkers abnormal, no cognitive impairments), and a symptomatic stage (biomarkers abnormal, cognitive impairments). The symptomatic stage can be further subdivided in a subjective cognitive impairments stage (AD-SCI), a mild cognitive impairment stage (AD-MCI), and a dementia stage (AD-dementia). Both the IWG criteria and NIA-AA recommendations deal with the asymptomatic and symptomatic stages of AD but the approaches and terminology differ (figure).

\section{IWG criteria}

The IWG proposes two sets of criteria. Both sets of criteria require assessment of AD biomarkers. The first set of criteria refers to subjects with asymptomatic $\mathrm{AD}$ and is referred to as 'preclinical AD'. In the 2010 revision, 'preclinical AD' is subdivided in 'asymptomatic at risk for $\mathrm{AD}^{\prime}$ and 'presymptomatic $\mathrm{AD}^{\prime}$ [5]. This latter category applies to asymptomatic subjects who are carrier of autosomal dominant AD mutations. The second set of criteria is for subjects with symptomatic $\mathrm{AD}$ and is referred to as 'AD'. Subjects who meet these criteria can be subclassified as 'prodromal AD' or 'predementia AD' (the AD-MCI stage in the figure) or 'AD dementia' (the AD-dementia stage in the figure). Persons with AD-SCI cannot yet be classified by the criteria. The authors indicate that further research is needed before it can be decided whether subjects with AD-SCI should be considered as having AD or as being asymptomatic at risk for $\mathrm{AD}[5]$.

\section{NIA-AA recommendations}

The NIA-AA proposes 3 sets of criteria in case biomarkers are available: one for the asymptomatic stage called 'preclinical $\mathrm{AD}^{2}$ ', one for the $\mathrm{AD}-\mathrm{MCI}$ stage called 'MCI due to $\mathrm{AD}$ ', and one for the AD-dementia stage called 'dementia due to AD'. In case no biomarkers can be used for the diagnosis, subjects should be classified according to clinical criteria. Persons with dementia can be diagnosed as probable or possible AD and persons with MCI as MCI. 


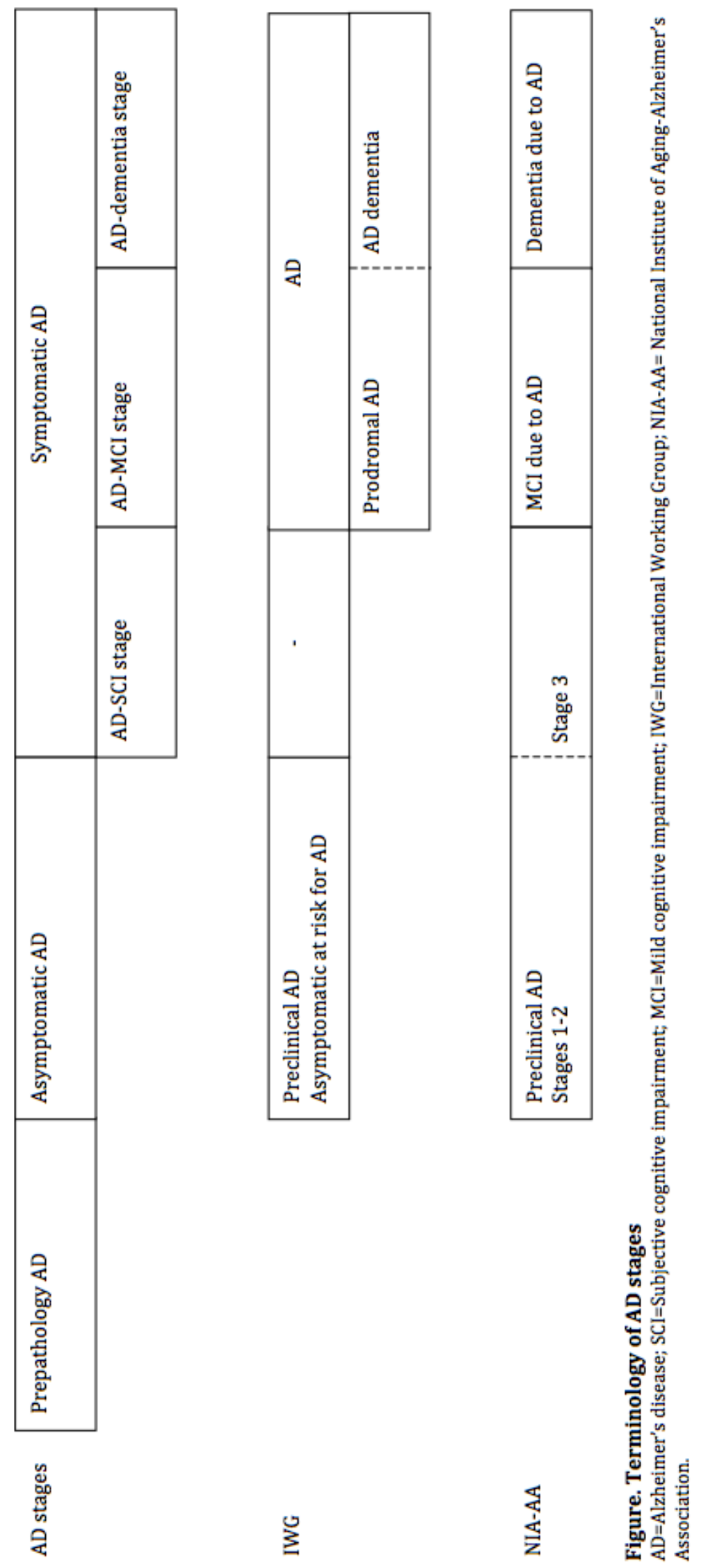




\section{COGNITIVE CRITERIA}

The main difference between the cognitive criteria in the two sets is in how they deal with non-memory impairments.

\section{IWG criteria}

The $\mathrm{AD}$ criteria require impairment on an episodic memory test that preferably has encoding specificity. Dementia is not necessary, although a subdivision in prodromal AD and $\mathrm{AD}$ dementia can be made as described above. In the 2010 update, persons with nonmemory impairments can meet the AD criteria as well but only if they have a well-defined syndrome such as primary progressive non-fluent aphasia, logopenic aphasia, frontal variant of AD, or posterior cortical atrophy. These subjects are referred to as atypical AD [5].

\section{NIA-AA recommendations}

In the NIA-AA criteria memory impairment is not necessary for the diagnosis. The criteria for $\mathrm{MCI}$ due to $\mathrm{AD}$ require $\mathrm{MCI}$, either amnestic or non-amnestic. These $\mathrm{MCI}$ criteria are similar to those published in 2004 [6-7]. The criteria for dementia due to AD require impairments in multiple cognitive domains and interference in activities of daily living. For the diagnosis of dementia, memory impairment is not necessary either.

\section{BIOMARKER CRITERIA}

Both sets of criteria rely essentially on the same biomarkers for AD pathology, i.e. amyloid beta 1-42 in cerebrospinal fluid (CSF), amyloid deposits as measured by PET scanning, tau in CSF, hippocampal atrophy, or hypoperfusion or hypometabolism as measured by PET or SPECT imaging. They differ in the way they how they combine these biomarkers for the diagnosis.

\section{IWG criteria}

In the original criteria biomarkers were subdivided according to modality (CSF, PET, SPECT, or MRI). In the 2010 revision biomarkers were classified as pathophysiological markers (markers for amyloid or tau) and topographical markers (other biomarkers) [5]. An abnormal score for any AD biomarker (pathophysiological or topographical) is sufficient to fulfill the biomarker criterion for $\mathrm{AD}$ (table 2). In subjects with preclinical $A D$, pathophysiological markers should be abnormal (table 1). 
Table 1. IWG and NIA-AA criteria for asymptomatic AD

\begin{tabular}{lllll}
\hline & IWG & NIA-AA & & \\
& & Stage 1 & Stage 2 & Stage 3 \\
\hline $\begin{array}{l}\text { Cognitive } \\
\text { criteria }\end{array}$ & No impairment & No impairment & No impairment & $\begin{array}{l}\text { Subtle cognitive } \\
\text { decline }\end{array}$ \\
$\begin{array}{l}\text { Biomarker } \\
\text { criteria }\end{array}$ & Amyloid or tau & Amyloid abnormal & Amyloid and injury & Amyloid and injury \\
& abnormal & & marker abnormal & marker abnormal \\
\hline
\end{tabular}

IWG=International Working Group; NIA-AA= National Institute of Aging-Alzheimer's Association.

Table 2. IWG and NIA-AA criteria for AD-MCI and AD-dementia

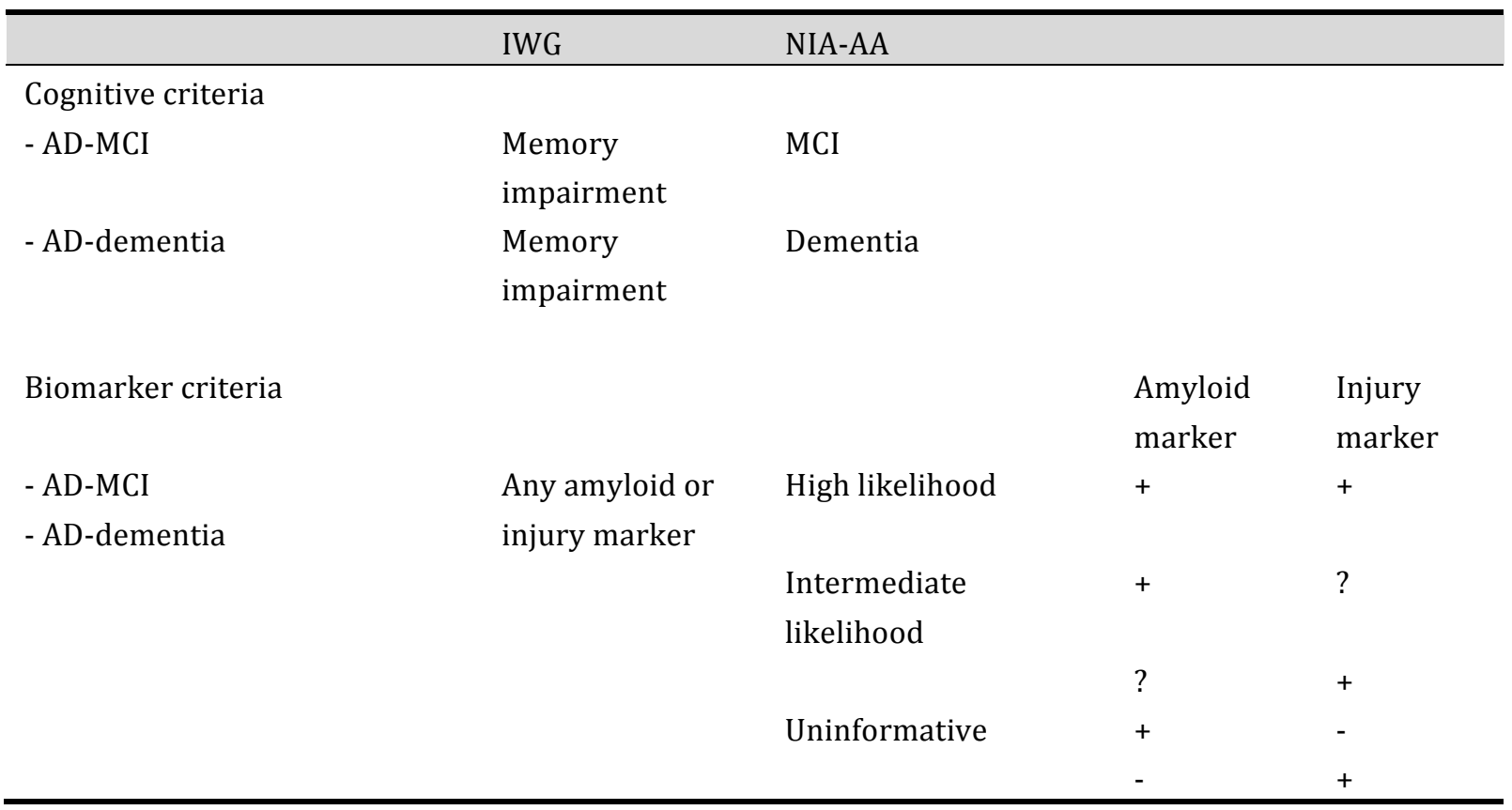

IWG=International Working Group; NIA-AA= National Institute of Aging-Alzheimer's Association; AD=Alzheimer's disease; $\mathrm{MCI}=$ Mild cognitive impairment. + = Abnormal; - = Normal; $?=$ Not tested.

\section{NIA-AA recommendations}

The NIA-AA criteria make a distinction between amyloid markers and neuronal injury markers (other biomarkers). Persons with preclinical AD are classified in three stages based on biomarkers and cognitive markers (table 1). Persons with abnormal amyloid markers only are in stage 1 , subjects with both abnormal amyloid and injury markers are in stage 2 . Persons with both abnormal amyloid and injury markers and subtle cognitive impairments, including SCI, are in stage 3.

Persons with MCI or dementia due to AD are classified based on amyloid and neuronal injury markers (table 2). If both amyloid and neuronal injury markers are abnormal, the likelihood for AD is high. If only amyloid or neuronal injury markers have been measured and the test is abnormal, the likelihood is intermediate. If the amyloid marker is abnormal and the neuronal injury marker normal or vice versa, the biomarkers are considered uninformative. 


\section{DISCUSSION}

\section{Strengths and limitations}

A strength of the IWG criteria for AD is that that they cover the clinical spectrum from MCI to dementia. This circumvents the arbitrary and sometimes difficult distinction between MCI and dementia. Limitations are the strong emphasis on memory impairment as nondemented subjects with AD may present with non-memory impairments [8-9]. Moreover, the term prodromal AD for subjects with AD-MCI may be confusing. The authors use this term to indicate that these subjects are prodromal to the dementia stage. However, 'prodromal' could be easily understood as prodromal to AD itself, although these subjects meet the IWG AD criteria.

A strength of the NIA-AA recommendations is the distinction between amyloid and injury markers in the criteria for symptomatic AD. Also the broad definition of cognitive impairment including non-amnestic MCI is useful. A limitation is the use of three sets of criteria for a single disease. This entails that the diagnosis change with disease progression, which may cause confusion to clinicians and patients. Another limitation is that the ranking of biomarkers in persons with MCI or dementia may be difficult to apply. Moreover, a substantial number of subjects may have 'uninformative' biomarker test results. Unpublished data from our own cohorts showed that $60 \%$ of the subjects with MCI had conflicting scores for CSF amyloid beta 1-42, CSF tau, and hippocampal atrophy.

A limitation of both sets of criteria is that in persons with MCI and dementia the amyloid and injury markers are given the same diagnostic accuracy for AD. The amyloid cascade hypothesis, however, states that amyloid pathology marks the start of AD and that neuronal injury is a secondary event [10]. Amyloid markers will therefore have a higher sensitivity for AD than injury markers while injury markers will have a higher specificity [11].

\section{Classification of subjects according to the criteria}

Most persons that meet the IWG criteria will also meet the NIA-AA criteria and vice versa. Subjects with non-amnestic MCI who meet the NIA-AA MCI due to AD criteria but not the IWG criteria may form the only exception. An important difference between the NIA-AA criteria and the IWG criteria is that the NIA-AA criteria make a subclassification based on biomarker results within each diagnostic group. For example, a person with MCI who meets the IWG criteria will meet the MCI due to AD NIA-AA criteria as well and will then be subclassified to have a high or intermediate likelihood for $\mathrm{AD}$ or to have uninformative biomarker results.

\section{Use of the criteria}

The current use of the criteria will be for research, as emphasized by both the IWG and the NIA-AA working group. Which set of criteria should be preferred will depend on the purpose of the study. For example, recent AD-MCI trials with amyloid modifying treatments selected subjects with amyloid markers [12-13]. These persons would meet the IWG AD 
criteria or the NIA-AA MCI due to AD criteria with intermediate likelihood for AD. In case a study requires including subjects with an increased risk for cognitive decline, a combination of amyloid and injury markers could be used, such as the NIA-AA MCI or dementia due to AD criteria with a high likelihood for AD [14-18].

\section{Future perspectives}

The new criteria are useful attempts to translate the results from AD biomarker studies into guidelines for diagnosis. Further research is needed to validate the criteria. It seems useful to reconsider the ranking of biomarkers in the criteria such that amyloid markers are used for the diagnosis and injury markers for the staging of the disease and for prognosis [14-18]. In the meantime, clinicians can apply biomarkers for diagnostic purposes outside the framework of diagnostic criteria. In persons with dementia biomarkers may for example help to differentiate between AD and frontotemporal lobar dementia [19]. In persons with $\mathrm{MCI}, \mathrm{AD}$ biomarkers may be tested if the patient asks to know what is the likely cause of the cognitive impairments [20]. Patients should not be tested for AD biomarkers with the only goal to classify them according to the new criteria. 


\section{REFERENCES}

1. Dubois B, Feldman HH, Jacova C, Dekosky ST, Barberger-Gateau P, Cummings J, et al. Research criteria for the diagnosis of Alzheimer's disease: revising the NINCDS-ADRDA criteria. Lancet Neurol 2007;6:734-746.

2. Albert MS, DeKosky ST, Dickson D, Dubois B, Feldman HH, Fox NC, et al. The diagnosis of mild cognitive impairment due to Alzheimer's disease: Recommendations from the National Institute on Aging and Alzheimer's Association workgroup. Alzheimer's and Dementia 2011.

3. McKhann GM, Knopman DS, Chertkow H, Hyman BT, Jack CR, Kawas CH, et al. The diagnosis of dementia due to Alzheimer's disease: Recommendations from the National Institute on Aging and the Alzheimer's Association workgroup. Alzheimer's and Dementia 2011.

4. Sperling RA, Aisen PS, Beckett LA, Bennett DA, Craft S, Fagan AM, et al. Toward defining the preclinical stages of Alzheimer's disease: Recommendations from the National Institute on Aging and the Alzheimer's Association workgroup. Alzheimer's and Dementia 2011.

5. Dubois B, Feldman HH, Jacova C, Cummings JL, Dekosky ST, Barberger-Gateau P, et al. Revising the definition of Alzheimer's disease: a new lexicon. Lancet Neurol 2010;9:1118-1127.

6. Winblad B, Palmer K, Kivipelto M, Jelic V, Fratiglioni L, Wahlund LO, et al. Mild cognitive impairment--beyond controversies, towards a consensus: report of the International Working Group on Mild Cognitive Impairment. Journal of Internal Medicine 2004;256:240-246.

7. Petersen RC. Mild cognitive impairment as a diagnostic entity. J Intern Med 2004;256:183-194.

8. Visser PJ, Verhey F, Knol DL, Scheltens P, Wahlund LO, Freund-Levi Y, et al. Prevalence and prognostic value of CSF markers of Alzheimer's disease pathology in patients with subjective cognitive impairment or mild cognitive impairment in the DESCRIPA study: a prospective cohort study. Lancet Neurol 2009;8:619-627.

9. Schneider JA, Arvanitakis Z, Leurgans SE, Bennett DA. The neuropathology of probable Alzheimer disease and mild cognitive impairment. Ann Neurol 2009;66:200-208.

10. Jack CR, Jr., Knopman DS, Jagust WJ, Shaw LM, Aisen PS, Weiner MW, et al. Hypothetical model of dynamic biomarkers of the Alzheimer's pathological cascade. Lancet Neurol 2010;9:119-128.

11. Vos S, Soininen H, Visser PJ, Blennow K, Verhey F, Burns L, et al. CSF and MRI biomarkers as predictors for Alzheimer's-type dementia in subjects with mild cognitive impairment. Alzheimer's \& Dementia 2011;7:S105

12. A Multicenter, Double Blind, Placebo-Controlled, Safety and Tolerability Study of BMS-708163 in Patients With Prodromal Alzheimer's Disease [online]. Available at: http://clinicaltrials.gov/ct2/show/NCT00890890. Accessed 23 July 2011.

13. A Study of Gantenerumab in Patients With Prodromal Alzheimer's Disease [online]. Available at: http://clinicaltrials.gov/ct2/show/NCT01224106. Accessed 23 July 2011.

14. van Rossum IA, Visser PJ, van der Flier W, Blankenstein MA, Scheltens P. Predictors of time to AD diagnosis in subjects with MCI. Alzheimer's \& Dementia 2010;6:S99.

15. Jack CR, Wiste HJ, Vemuri P, Weigand SD, Senjem ML, Zeng G, et al. Brain beta-amyloid measures and magnetic resonance imaging atrophy both predict time-to-progression from mild cognitive impairment to Alzheimer's disease. Brain 2010;133:3336-3348.

16. Hansson O, Buchhave P, Zetterberg H, Blennow K, Minthon L, Warkentin S. Combined rCBF and CSF biomarkers predict progression from mild cognitive impairment to Alzheimer's disease. Neurobiol Aging 2007.

17. Wallin AK, Blennow K, Zetterberg H, Londos E, Minthon L, Hansson O. CSF biomarkers predict a more malignant outcome in Alzheimer disease. Neurology 2010;74:1531-1537. 
18. Kester MI, van der Vlies AE, Blankenstein MA, Pijnenburg YA, van Elk EJ, Scheltens P, et al. CSF biomarkers predict rate of cognitive decline in Alzheimer disease. Neurology 2009;73:1353-1358.

19. Engler H, Santillo AF, Wang SX, Lindau M, Savitcheva I, Nordberg A, et al. In vivo amyloid imaging with PET in frontotemporal dementia. Eur J Nucl Med Mol Imaging 2008;35:100-106.

20. Porteri C, Galluzzi S, Geroldi C, Frisoni GB. Diagnosis disclosure of prodromal Alzheimer diseaseethical analysis of two cases. Can J Neurol Sci 2010;37:67-75. 


\section{CHAPTER 8}

PRECLINICAL ALZHEIMER'S DISEASE AND ITS OUTCOME:

A LONGITUDINAL COHORT STUDY

The Lancet Neurology 2013;12(10):957-65

Stephanie J.B. Vos, Chengjie Xiong, Pieter Jelle Visser, Mateusz S. Jasielec, Jason Hassenstab, Elizabeth A. Grant, Nigel J. Cairns, John C. Morris, David M. Holtzman, Anne M. Fagan 


\section{ABSTRACT}

\section{Background}

New research criteria for preclinical Alzheimer's disease have been proposed, which include stages for cognitively normal individuals with abnormal amyloid markers (stage 1), abnormal amyloid and neuronal injury markers (stage 2), or abnormal amyloid and neuronal injury markers and subtle cognitive changes (stage 3). We aimed to investigate the prevalence and long-term outcome of preclinical Alzheimer's disease according to these criteria.

\section{Methods}

Participants were cognitively normal (clinical dementia rating $[\mathrm{CDR}]=0$ ) communitydwelling volunteers aged at least 65 years who were enrolled between 1998 and 2011 at the Washington University School of Medicine (MO, USA). CSF amyloid- $\beta 1-42$ and tau concentrations and a memory composite score were used to classify participants as normal (both markers normal), preclinical Alzheimer's disease stage 1-3, or suspected nonAlzheimer pathophysiology (SNAP, abnormal injury marker without abnormal amyloid marker). The primary outcome was the proportion of participants in each preclinical AD stage. Secondary outcomes included progression to CDR at least 0.5 , symptomatic Alzheimer's disease (score of at least 0.5 for memory and at least one other domain and cognitive impairments deemed to be due to Alzheimer's disease) and mortality. We undertook survival analyses using subdistribution and standard Cox hazards models and linear mixed models.

\section{Findings}

Of 311 participants, 129 (41\%) were classed as normal, 47 (15\%) as stage 1, $36(12 \%)$ as stage $2,13(4 \%)$ as stage 3 , and $72(23 \%)$ as SNAP, and $14(5 \%)$ remained unclassified. The 5-year progression rate to CDR at least 0.5, symptomatic Alzheimer's disease was $2 \%$ for participants classed as normal, $11 \%$ for stage $1,26 \%$ for stage $2,56 \%$ for stage 3 , and $5 \%$ for SNAP. Compared with individuals classed as normal, participants with preclinical Alzheimer's disease had an increased risk of death after adjusting for covariates (hazard ratio $6.2,95 \%$ CI 1.1-35.0; $\mathrm{p}=0.040$ ).

\section{Interpretation}

Preclinical Alzheimer's disease is common in cognitively normal elderly people and is associated with future cognitive decline and mortality. Thus, preclinical Alzheimer's disease could be an important target for therapeutic intervention. 


\section{INTRODUCTION}

Alzheimer's disease (AD) starts with a preclinical phase in which AD neuropathological abnormalities begin to accumulate but cognitive ability is normal. ${ }^{1-3}$ Now that biomarkers for AD have become available, identification of preclinical AD in vivo in cognitively normal individuals is possible. ${ }^{4}$ Information regarding the occurrence and outcome of preclinical AD is crucial for the understanding of $\mathrm{AD}$ pathophysiology and the design of secondary prevention trials.

Research criteria for preclinical AD have been proposed by the Preclinical Working Group of the National Institute on Aging (NIA) and Alzheimer's Association (AA). ${ }^{5}$ The NIAAA criteria for preclinical AD propose ordered stages for cognitively normal individuals with abnormal amyloid markers (stage 1), abnormal amyloid and neuronal injury markers (stage 2), and abnormal amyloid and neuronal injury markers and subtle cognitive changes (stage 3). ${ }^{5}$ In a 2012 study in which structural and amyloid imaging markers were used to categorise individuals according to these stages, ${ }^{6}$ the rate of short-term ( 1 year) progression to mild cognitive impairment (MCI) or dementia increased with advancing preclinical AD stage.

The aim of this study was to identify the prevalence and long-term outcome of preclinical $\mathrm{AD}$ according to these criteria in a cohort of cognitively normal individuals. We used CSF markers to define NIA-AA preclinical AD stages and assessed the long-term cognitive and mortality outcomes of participants in each stage. We also tested whether the proportion and cognitive outcome of preclinical AD were affected by age or APOE genotype.

\section{METHODS}

\section{Participants}

Participants were cognitively normal community-dwelling volunteers enrolled between June, 1998, and September, 2011, in longitudinal studies of memory and ageing at the Knight Alzheimer's Disease Research Center (KADRC) of the Washington University School of Medicine (St Louis, MO, USA). Details of recruitment and assessment methods for these participants have been published. ${ }^{7}$ Participants in the KADRC cohort were living independently in the community at study entry and underwent annual clinical assessment unless prevented by death, illness, refusal, or relocation from St Louis. Participants were selected from the larger KADRC cohort based on the following criteria: completion of baseline cognitive and CSF assessment; baseline clinical dementia rating (CDR) score of 0; at least 65 years of age at the time of lumbar puncture; at least one annual clinical follow-up assessment; and good general health. The Human Research Protection Office at Washington University School of Medicine approved the KADRC studies, including the Healthy Aging and Senile Dementia study (P01AG003991), the Alzheimer's Disease Research Center study (P50AG05681), and the Antecedent Biomarkers for AD: the Adult Children Study (P01AG026276). Written informed consent was obtained from all participants at enrolment. 


\section{Procedures}

Participants underwent annual cognitive assessment, which included CDR and CDR sum of boxes (CDR-SB), ${ }^{8}$ mini-mental state examination (MMSE), and a psychometric test battery. ${ }^{9}$ The CDR is a global dementia staging system that assesses the presence or absence of dementia and, when present, its severity. The global CDR stages are 0 , indicating cognitive normality, and $0.5,1,2$, and 3 , indicating very mild impairment or very mild dementia, and mild, moderate, and severe dementia, respectively. ${ }^{10}$ The CDR-SB is a more quantitative representation of cognitive impairment than the global CDR and is derived directly from individual ratings in six cognitive and functional domains, or boxes (memory, orientation, judgment and problem solving, community affairs, home and hobbies, and personal care). The CDR-SB is the total score of all the separate boxes (range $0-18$, with 0 as the best score). Participants with a CDR score of 0 typically have scores of 0 on all box scores; however, a global CDR score of 0 can also be assigned in the presence of one box score of 0.5 in a nonmemory domain. Baseline CDR score and diagnosis were assigned by trained clinicians and were based on the cognitive assessment closest to the time of the lumbar puncture.

We used CSF markers to define NIA-AA preclinical AD stages. CSF amyloid- $\beta_{1-42}\left(A \beta_{1-}\right.$ 42) was used as a marker of amyloid and CSF tau was used as a marker of neuronal injury. CSF samples (20-25 mL) were collected once at study entry at $0800 \mathrm{~h}$ after overnight fasting. Lumbar punctures (lumbar vertebrae L4/L5) were done by trained neurologists using a 22-gauge atraumatic Sprotte spinal needle (Pajunk Medical Systems, Norcross, GA, USA). Samples were gently inverted to avoid possible gradient effects, briefly centrifuged at low speed, and aliquoted $(0.5 \mathrm{~mL})$ into polypropylene tubes before being frozen at $-84^{\circ} \mathrm{C}$. Samples were analysed for total tau ( $\mathrm{t}$-tau), phosphorylated $\operatorname{tau}_{181}\left(\mathrm{p}-\mathrm{tau}_{181}\right)$, and $\mathrm{A} \beta_{1-42}$ by ELISA (INNOTEST; Innogenetics, Ghent, Belgium).

CSF markers were dichotomised (normal or abnormal) by defining a cutoff that could best differentiate participants in our cohort who had CDR 0 at baseline from those in an independent cohort who had CDR 0.5, symptomatic AD (for demographics of this cohort, see appendix), on the basis of the Youden index (sensitivity+specificity-1). The resultant optimum cutoffs for abnormal were less than $459 \mathrm{pg} / \mathrm{mL}$ for $A \beta_{1-42}$, greater than $339 \mathrm{pg} / \mathrm{mL}$ for t-tau, and greater than $67 \mathrm{pg} / \mathrm{mL}$ for $\mathrm{p}$-tau 181 .

The NIA-AA criteria for preclinical AD do not define the subtle cognitive changes needed for classification as stage 3 . Since episodic memory is usually the earliest cognitive domain to be affected in $\mathrm{AD},{ }^{11,12}$ we used an episodic memory composite score as the measure of cognition to define stage 3 . The composite score was based on factor analyses ${ }^{9}$ and consisted of the sum of the three free recall trials from the Buschke free and cued selective reminding test, ${ }^{13}$ total scores from the easy and hard trials of associate learning from the Wechsler memory scale-revised, ${ }^{14}$ and the total number of correctly recalled units from the Wechsler memory scale-revised logical memory immediate recall test. Raw scores from each test were converted to $\mathrm{Z}$ scores using a normative reference sample of participants from the KADRC who were cognitively normal at enrolment and who did not progress to a dementia diagnosis during follow-up (ie, remained CDR 0). ${ }^{15}$ The normative 
reference sample, as described in detail by Johnson and colleagues, ${ }^{15}$ was composed of participants who were enrolled in ongoing studies at the KADRC between Oct 1, 1979, and Dec 31, 2006. 82 (26\%) of the 311 participants in the present study contributed cognitive scores (almost exclusively from annual visits before CSF collection) to the normative reference sample. The mean of the three tests was used to create a composite episodic memory score and was converted again into a $\mathrm{Z}$ score within the population. The cutoff at the lowest tenth percentile of the distribution in our sample (less than $-1.25 \mathrm{SD}$ ) was applied to signify memory impairment.

At baseline, participants were classified as normal if both episodic memory and CSF markers met our criteria for normal, in stage 1 if only $A \beta_{1-42}$ was abnormal, in stage 2 if $A \beta_{1-}$ 42 and either t-tau or p-tau 181 were abnormal, and in stage 3 if additionally the participant's cognitive ability was below the memory test threshold (panel 1). Participants were classified in the suspected non-Alzheimer pathophysiology (SNAP) group if they had abnormal t-tau or $\mathrm{p}$-tau 181 in the presence of normal $A \beta_{1-42}$, regardless of episodic memory ability (panel 1). ${ }^{16}$ Participants who did not fit within one of the groups were included in the unclassified group and, in view of the uncertainty of their classification, were excluded from the main analyses.

\section{Panel 1. Glossary of preclinical AD stages and symptomatic AD}

\begin{tabular}{ll}
\hline Normal group & CDR 0 , amyloid $(-)$, neural injury $(-)$, subtle cognitive decline $(-)$ \\
Preclinical AD stage 1 & CDR 0, amyloid $(+)^{2}$, neural injury $(-)$, subtle cognitive decline $(-)$ \\
Preclinical AD stage 2 & CDR 0, amyloid $(+)$, neural injury $(+)^{3}$, subtle cognitive decline $(-)$ \\
Preclinical AD stage 3 & CDR 0, amyloid $(+)$, neural injury $(+)$, subtle cognitive decline $(+)^{4}$ \\
SNAP group 5 & CDR 0, amyloid $(-)$, neural injury $(+)$, subtle cognitive decline $(-/+)$ \\
Unclassified group & CDR 0, amyloid $(-/+)$, neural injury $(-)$, subtle cognitive decline $(+)$ \\
Symptomatic AD & CDR $>0$, memory and at least one other domain received a score of $\geq 0.5$ and \\
& the clinician felt the cognitive impairments to be due to AD (probable AD), no \\
& reference to biomarkers
\end{tabular}

${ }^{1}$ CDR 0, Clinical Dementia Rating=0, no dementia, ${ }^{2}$ Amyloid, CSF Ab42 (+) <459pg/mL, 3Neural injury, CSF tau (+) $>339 \mathrm{pg} / \mathrm{mL}$ or ptau181 (+) $>67 \mathrm{pg} / \mathrm{mL}$, ${ }^{4}$ Subtle cognitive decline, episodic memory composite score (+) in the lowest $10^{\text {th }}$ percentile, 5 SNAP, Suspected Non-Alzheimer Pathophysiology, ${ }^{6} \mathrm{CDR}>0, \mathrm{CDR} 0.5=$ very mild dementia, CDR $1=$ mild dementia, $\operatorname{CDR} 2=$ moderate dementia, $\operatorname{CDR} 3=$ severe dementia.

The primary outcome measure was the proportion of participants in each preclinical AD stage, as defined by CSF markers and scores on episodic memory tests. Secondary outcome measures were cognitive decline on the CDR-SB and MMSE, progression to CDR at least 0.5, symptomatic $\mathrm{AD}$ at the latest available follow-up before dropout, and mortality.

In our KADRC research cohort, the clinical diagnosis of AD in individuals with a CDR score of 0.5 or greater is based on criteria from the National Institute of Neurological and Communicative Disorders and Stroke and the Alzheimer's Disease and Related Disorders Association, ${ }^{17}$ in accordance with standard protocols. ${ }^{7}$ In individuals with a CDR score of 0.5 , $\mathrm{AD}$ was diagnosed if a score of at least 0.5 was given for memory and at least one other 
domain and the clinician deemed the cognitive impairments to be due to AD (probable AD; referred to as CDR 0.5, symptomatic AD). CDR 0.5, symptomatic AD differs from MCI as defined in the criteria of $\mathrm{MCI}$ due to $\mathrm{AD}$ or prodromal $\mathrm{AD}, 18,19$ although it is similar (appendix). As part of the assessment to define whether a participant was cognitively impaired with or without symptomatic $\mathrm{AD}$, a thorough, informant-based and participantbased interview was completed. Cognitive examination, consisting of assessment of recent and long-term memory, executive function, reasoning, language, and visuospatial function, was also undertaken by trained clinicians. The CDR rating and diagnosis are based on both present and historical cognitive ability. In contrast to $\mathrm{MCI}$, an absolute cutoff score on a specific cognitive test is not used to define the presence of cognitive impairment. Moreover, classification as CDR 0.5, symptomatic AD requires clinical change in two cognitive domains, whereas subjective complaints and impairment in any cognitive domain is sufficient to meet the criteria for MCI (appendix).

In a subset of participants for whom autopsy samples were available, neuropathological examination was undertaken using established protocols. ${ }^{20} \mathrm{AD}$ neuropathological changes were rated using the NIA-AA guidelines. ${ }^{21}$ We also tested whether the prevalence and long-term outcome of preclinical AD were affected by age or APOE genotype. TaqMan assays (Applied Biosystems, Foster City, CA, USA) for both ABI\#C_3084793_20 and ABI\#C_904973_10 were used for APOE genotyping, as described previously. ${ }^{22}$

\section{Statistical analysis}

Baseline differences between the stages were analysed using ANOVA for continuous variables and Fisher's exact tests and logistic regression models for categorical variables. Missing data from cognitive tests at follow-up were modelled with mixed models. We first undertook an omnibus test for joint significance of the stage variables and proceeded with subgroup analyses only if this overall test was statistically significant. We did competingrisks survival analyses using Fine and Gray's ${ }^{23}$ subdistribution hazards model (subdistribution hazards ratio [SHR]) to investigate the predictive accuracy of the preclinical $A D$ stages for progression to $C D R$ at least 0.5 , symptomatic $A D$ during the available follow-up period, with normal individuals as a reference group, uncorrected and corrected for baseline age, sex, education, and APOE genotype. Unlike standard Cox hazards models that usually treat mortality as censoring, these models regard mortality as a competing event that can impede progression to symptomatic AD. Standard Cox proportional hazards models (hazard ratio [HR]) were used to assess the predictive capacity of preclinical AD stages for mortality during the follow-up period, in both unadjusted and adjusted analyses. The relation between the stages and rate of change in CDR-SB and MMSE over time were assessed with general linear mixed models including linear time effects, ${ }^{24}$ adjusted for baseline age, sex, education, and APOE genotype. Analyses included baseline score and all available follow-up scores. The final models were specified with a random intercept and slope, because these models provided the best measures on Akaike's 
information criterion ${ }^{25}$ for analysis of the corresponding clinical and cognitive measures compared with models with other covariance structures. Specific values for each covariate were used to create adjusted plots for the survival analyses and mixed models. Predicted curves or slopes for each of the AD stage groups were plotted within each combination of APOE $\varepsilon 4$ and sex with age (72.6 years) and education (15.5 years) fixed at the sample means. These means represent the total group of participants, excluding the unclassified group because this group was not included in these analyses. Subdistribution hazards models were implemented using the STCRREG command in STATA 12 (Stata, College Station, TX, USA). All other statistical analyses were done with SPSS version 19.0 (Chicago, IL, USA), with significance set at $\mathrm{p}<0.05$.

\section{RESULTS}

Table 1 lists demographics and baseline characteristics. 129 of 311 (41\%) participants were classified in the normal group, $47(15 \%)$ as stage 1, $36(12 \%)$ as stage $2,13(4 \%)$ as stage 3 , $72(23 \%)$ as being in the SNAP group, and $14(5 \%)$ remained unclassified. The appendix shows the distribution of participants across the stages. MMSE and memory scores were lower in stage 3 than in the other groups (table 1). Preclinical AD (stage 1-3) was more prevalent in individuals older than 72 years (median age of sample) than in those aged 72 years or younger ( $37 \%$ vs $26 \%$; $=0.044$ ) and was more prevalent in APOE $\varepsilon 4$ carriers than in non-carriers ( $47 \%$ vs 23\%; $<<0.0001$; appendix). The mean interval between lumbar puncture and the closest cognitive assessment was 2.6 months (SD 2.1).

110 (35\%) participants were available at 5 years of follow-up and 14 (5\%) were available at 10 years. 39 patients (13\%) were lost to follow-up and 20 (6\%) died during follow-up. After a median follow-up of 3.9 years (range 1-15), progression to CDR at least 0.5 , symptomatic $\mathrm{AD}$ had occurred in two (2\%) participants in the group classed as normal, six $(13 \%)$ in stage 1 , nine $(25 \%)$ in stage 2 , seven $(54 \%)$ in stage 3 , four $(6 \%)$ in the SNAP group, and four (29\%) in the unclassified group (table 2). Of the 32 participants who progressed, 22 (69\%) were diagnosed with CDR 0.5, symptomatic AD at their last follow-up, six (19\%) had CDR 1, symptomatic AD, and four (13\%) had CDR 2, symptomatic AD. 


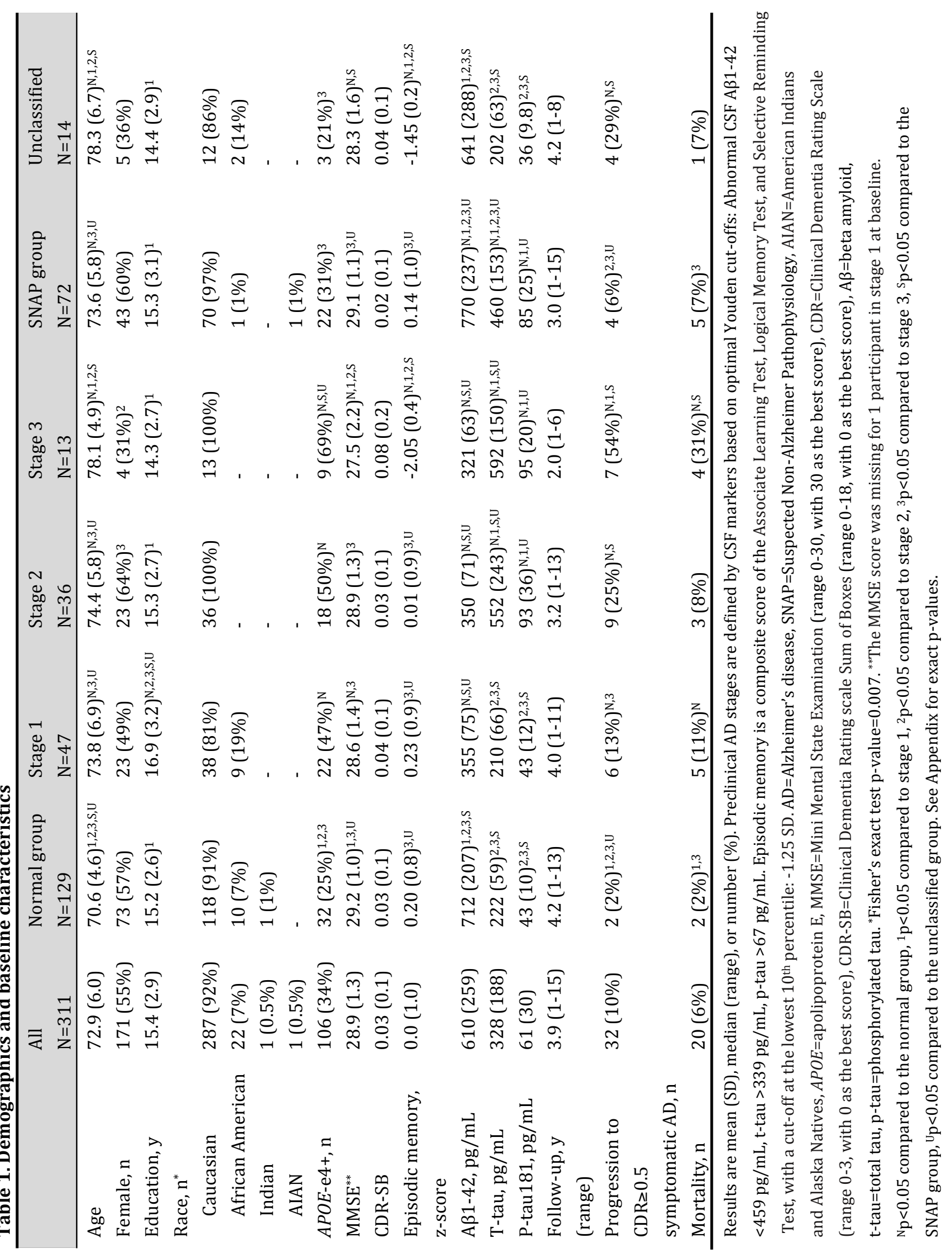




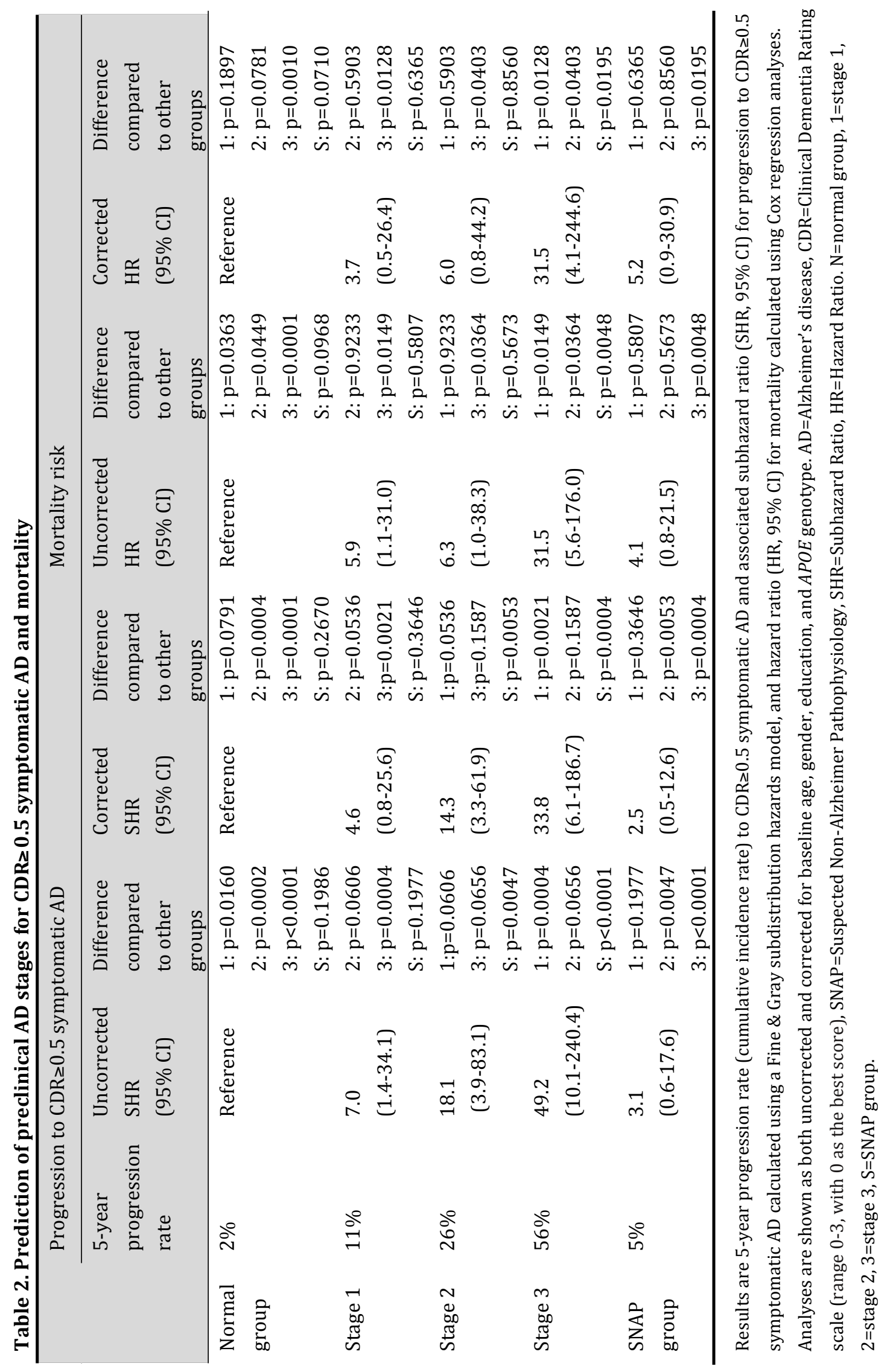


Survival analyses showed that, taking into account mortality, participants in each preclinical AD stage had a higher risk of progression to CDR at least 0.5, symptomatic AD than did participants classed as normal (stage 1 SHR 7.0, 95\% CI 1.4-34.1, p=0.016; stage 2 SHR 18.1, 3.9-83.1, p=0.0002; stage 3 SHR 49.2, 10.1-240.4, $<<0.0001$; table 2, figure). Preclinical AD stages also differed from each other, with more severe stages associated with higher risk of progression to symptomatic $\mathrm{AD}$, although the difference between stages 2 and 3 was not significant $(\mathrm{p}=0.066)$. The progression rate of participants in the SNAP group did not differ from that of individuals classed as normal $(p=0.20)$. Only plots for APOE $\varepsilon 4$ negative women are shown because this is the most populous group of the four combinations from the two dichotomous factors, APOE $\varepsilon 4$ and sex. However, effects of preclinical AD stages (either on risk of converting to a higher CDR or on rate of change in MMSE) remained the same regardless of the combinations of APOE $\varepsilon 4$ and sex. After correction for covariates, results remained essentially the same, except that progression in stage 1 was no longer different from that of individuals classed as normal (SHR 4.6, 95\% CI 0.8-25.6, p=0.079), which was mainly driven by the correction for age (data not shown). The estimated 5 -year progression (cumulative incidence) rate to $\mathrm{CDR}$ at least 0.5 , symptomatic $\mathrm{AD}$ was $2 \%$ for participants classed as normal, $11 \%$ for those in stage $1,26 \%$ for those in stage $2,56 \%$ for those in stage 3 , and $5 \%$ for those in the SNAP group. The risk of progression was not different between older ( $>72$ years) and younger ( $\leq 72$ years) individuals with preclinical AD (SHR 2.0, 95\% CI $0.7-5.5 ; \mathrm{p}=0.19$ ) or between APOE $\varepsilon 4$ carriers and non-carriers with preclinical AD (SHR 1.1, $0.5-2.6, \mathrm{p}=0.76$; appendix).

$20(6 \%)$ participants died during follow-up (table 1). Compared with individuals classed as normal, participants with preclinical AD (stage 1-3) had an increased risk of death after adjusting for covariates (HR 6.2, 95\% CI 1.1-35.0; $\mathrm{p}=0.040$ ), which increased with advancing stage (table 2). There was weak evidence of a difference in risk of death in the SNAP group compared with the normal group (HR after adjustment 5.2, 95\% CI 0.930.9; $\mathrm{p}=0.071$ ). Of the nine participants with preclinical AD who underwent autopsy, eight received a neuropathological diagnosis of $A D$ with intermediate-to-high neuropathological $\mathrm{AD}$ change and one received a diagnosis of $\mathrm{AD}$ with low neuropathological AD change (table 3). Three of four participants with SNAP who underwent autopsy had low level neuropathological AD change according to NIA-AA criteria; thus this pathological change is unlikely to explain the cognitive impairments. Of these three individuals with SNAP, two were in NIA-AA $A \beta$ stage 1 and one was in NIA-AA A $\beta$ stage 2 but this individual had a low neurofibrillary tangle score and vascular comorbidity. ${ }^{21}$ All participants with SNAP had a neuritic plaque score of 0 . Other coexisting pathological abnormalities in these individuals were minor and were deemed unlikely to have contributed substantially to the cognitive status. The time to death after baseline lumbar puncture ranged from 2 to 11 years for all participants; therefore, AD pathology might have accumulated by the time of autopsy.

The annual rate of increase in CDR-SB was higher in each preclinical stage than in the normal group (stage $1 \mathrm{p}=0.029$, stage $2 \mathrm{p}<0.0001$, stage $3 \mathrm{p}=0.0009$ ), in stages 2 and 3 than in the SNAP group (stage $2 \mathrm{p}=0.0021$ and stage $3 \mathrm{p}=0.0048$ ), and in stage 3 compared with 
stage 1 ( $\mathrm{p}=0.037$; table 4 ; appendix). The annual rate of decrease in the MMSE was higher in participants in stages 2 and 3 than in participants in the normal group (stage $2 p=0.0021$, stage $3 \mathrm{p}=0.023$ ) and in those in the SNAP group (stage $2 \mathrm{p}=0.0047$, stage $3 \mathrm{p}=0.028$; table 4; appendix). The appendix shows individual cognitive trajectories on the CDR-SB and MMSE.
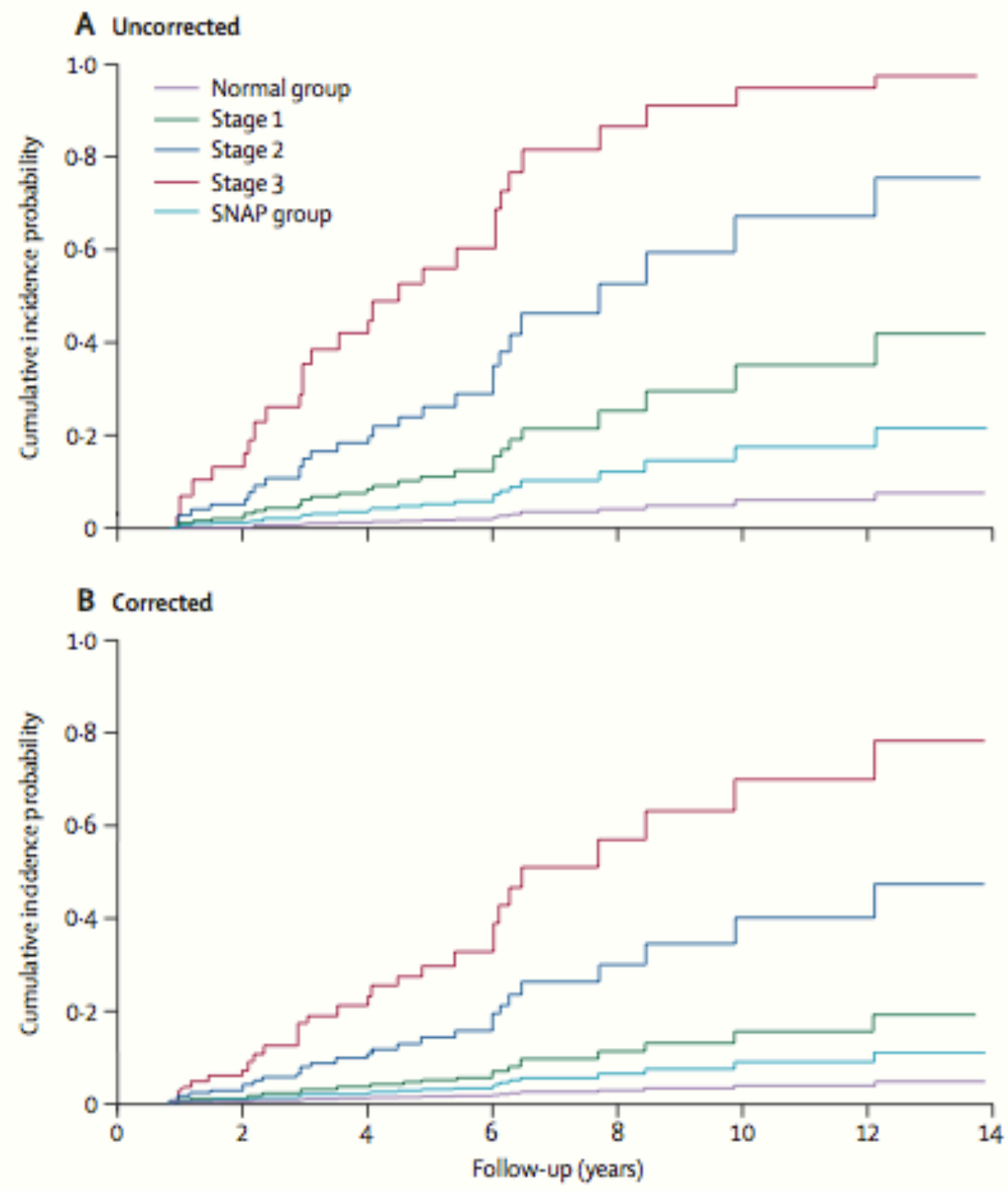

Number of participants

$\begin{array}{rrrrrrrrr}\text { Normal } & 129 & 112 & 73 & 29 & 10 & 4 & 1 & 0 \\ \text { Stage 1 } & 47 & 36 & 28 & 16 & 4 & 3 & 0 & 0 \\ \text { Stage 2 } & 36 & 31 & 15 & 9 & 5 & 1 & 1 & 0 \\ \text { Stage 3 } & 13 & 10 & 5 & 2 & 0 & 0 & 0 & 0 \\ \text { SNAP } & 72 & 61 & 28 & 15 & 11 & 6 & 6 & 2\end{array}$

\section{Figure. Progression to CDR at least 0.5, symptomatic AD by preclinical AD stage}

Cumulative incidence probability of $\mathrm{CDR}$ at least $0 \cdot 5$, symptomatic AD for each preclinical AD stage (A) uncorrected for covariates and (B) corrected for age, sex, education, and APOE genotype. $\mathrm{AD}=$ Alzheimer's disease. $\mathrm{CDR}=$ clinical dementia rating scale (range $0-3$, with 0 as the best score). SNAP=suspected non-Alzheimer pathophysiology. 


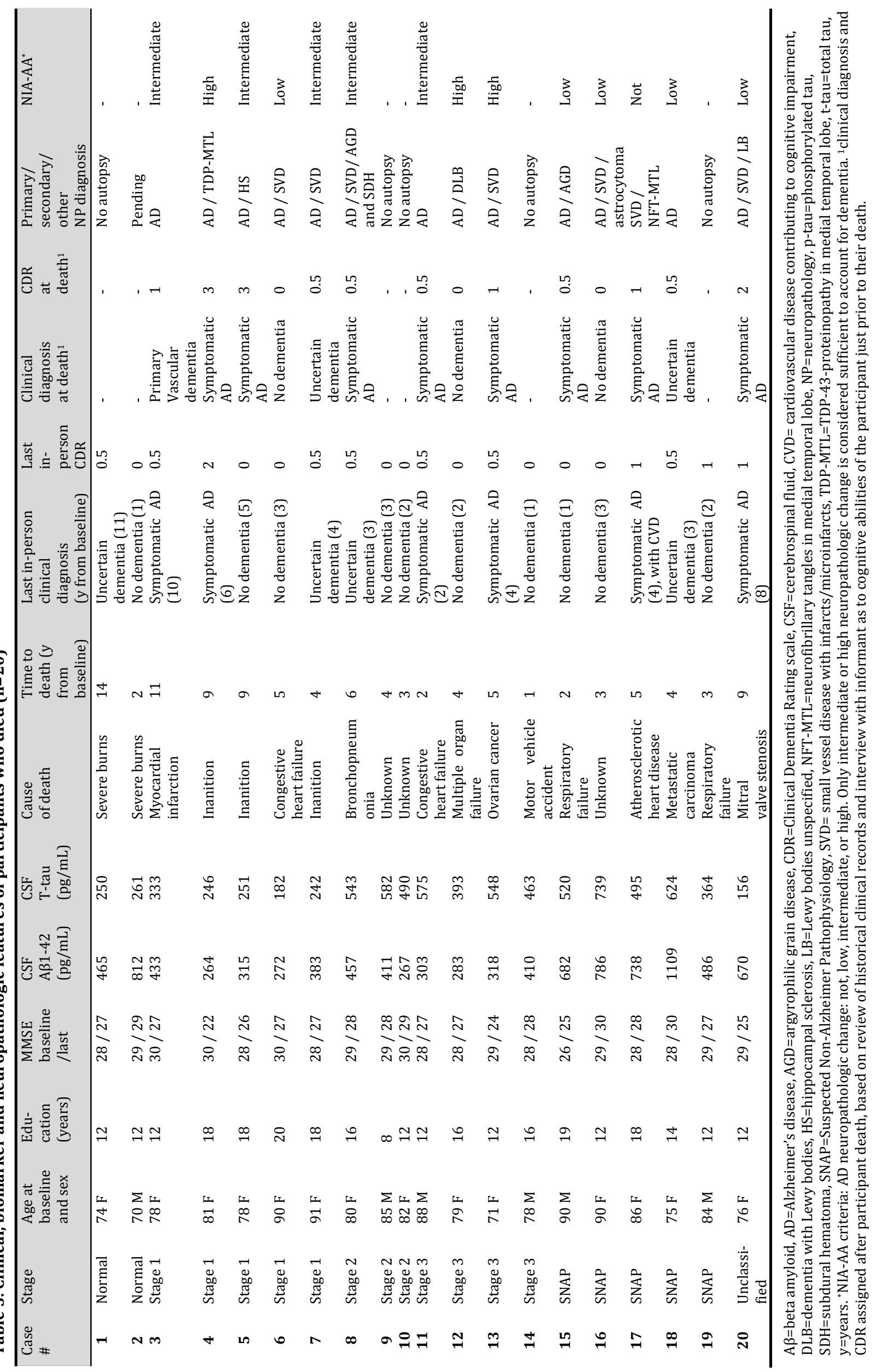


Table 4. Annual rate of change in CDR-SB and MMSE according to preclinical AD stage

\begin{tabular}{|c|c|c|c|c|c|c|}
\hline Stages & $\begin{array}{l}\text { Slope } \\
\text { CDR-SB }\end{array}$ & $\begin{array}{l}\text { P-value } \\
\text { slope }\end{array}$ & $\begin{array}{l}\text { Difference } \\
\text { compared to } \\
\text { other groups }\end{array}$ & $\begin{array}{l}\text { Slope } \\
\text { MMSE }\end{array}$ & $\begin{array}{l}\text { P-value } \\
\text { slope }\end{array}$ & $\begin{array}{l}\text { Difference } \\
\text { compared } \\
\text { other groups }\end{array}$ \\
\hline \multirow[t]{4}{*}{ Normal group } & $0.03(0.03)$ & $\mathrm{P}=0 \cdot 2784$ & $1: p=0 \cdot 0289$ & $-0.01(0.03)$ & $\mathrm{P}=0.6955$ & $1: p=0 \cdot 2324$ \\
\hline & & & $2: p<0.0001$ & & & $2: p=0.0021$ \\
\hline & & & $3: p=0.0009$ & & & $3: p=0.0233$ \\
\hline & & & $S: p=0 \cdot 3870$ & & & $S: p=0.9857$ \\
\hline \multirow[t]{3}{*}{ Stage 1} & $0.15(0.04)$ & $\mathrm{P}=0 \cdot 0014$ & $2: p=0 \cdot 0638$ & $-0.09(0.05)$ & $\mathrm{P}=0.0966$ & $2: p=0.0655$ \\
\hline & & & $3: p=0 \cdot 0373$ & & & $3: p=0 \cdot 1019$ \\
\hline & & & $S: p=0 \cdot 2096$ & & & $S: p=0 \cdot 2925$ \\
\hline \multirow[t]{3}{*}{ Stage 2} & $0.28(0.05)$ & $P<0 \cdot 0001$ & $1: p=0.0638$ & $-0.24(0.06)$ & $P=0 \cdot 0003$ & $1: p=0.0655$ \\
\hline & & & $3: p=0 \cdot 3848$ & & & $3: p=0.6227$ \\
\hline & & & $S: p=0 \cdot 0021$ & & & $S: p=0 \cdot 0047$ \\
\hline \multirow[t]{3}{*}{ Stage 3} & $0 \cdot 37(0 \cdot 10)$ & $\mathrm{P}=0 \cdot 0002$ & $1: p=0 \cdot 0373$ & $-0 \cdot 31(0 \cdot 12)$ & $\mathrm{P}=0 \cdot 0145$ & $1: p=0 \cdot 1019$ \\
\hline & & & $2: p=0 \cdot 3848$ & & & $2: p=0.6227$ \\
\hline & & & $S: p=0 \cdot 0048$ & & & $S: p=0 \cdot 0279$ \\
\hline \multirow[t]{3}{*}{ SNAP group } & $0.07(0.04)$ & $P=0 \cdot 0685$ & $1: p=0.2096$ & $-0.01(0.04)$ & $P=0.7578$ & $1: p=0 \cdot 2925$ \\
\hline & & & $2: p=0 \cdot 0021$ & & & $2: p=0 \cdot 0047$ \\
\hline & & & $3: p=0 \cdot 0048$ & & & $3: p=0 \cdot 0279$ \\
\hline
\end{tabular}

Data are slopes (SE) corrected for age, gender, education, and APOE genotype and comparison to other groups. $\mathrm{AD}=$ Alzheimer's disease, $\mathrm{CDR}-\mathrm{SB}=$ Clinical Dementia Rating scale Sum of Boxes (range 0-18, with 0 as the best score), MMSE=Mini Mental State Examination (range 0-30, with 30 as the best score), SNAP=Suspected NonAlzheimer Pathophysiology. N=normal group, 1=stage 1, 2=stage 2, 3=stage 3, S=SNAP group.

\section{DISCUSSION}

In this study, we show that preclinical AD can be defined by CSF markers, is common in individuals aged at least 65 years, and is associated with an increased risk of cognitive decline, progression to CDR at least 0.5 , symptomatic AD, and mortality (panel 2).

$31 \%$ of participants in our cohort had preclinical AD (stages 1-3), which is consistent with findings from clinicopathological studies ${ }^{1-3}$ and the population-based Mayo Clinic Study of Aging (MCSA), which used imaging measures (appendix). ${ }^{16}$ The validity of our biomarkerbased diagnosis of preclinical AD was further supported by the finding that eight of nine participants with preclinical $A D$ who underwent autopsy had intermediate-to-high $A D$ neuropathological changes. The distribution across the preclinical AD stages was also similar to that reported in the MCSA. ${ }^{16}$

Individuals with preclinical $\mathrm{AD}$ progressed faster to $\mathrm{CDR}$ at least 0.5 , symptomatic $\mathrm{AD}$ than did those in the normal and SNAP groups. Progression rates differed between the preclinical AD stages; thus, stages 1, 2, and 3 represent different and progressive disease severities. Findings from the MCSA also showed an increased rate of cognitive decline with advancing stage, although only 1 year of follow-up data were reported. ${ }^{6}$ 
Mortality risk was higher in participants with preclinical AD than in those in the normal group and also increased with advancing stage. To our knowledge, no other studies have examined mortality risk in preclinical $\mathrm{AD}$, but our findings are consistent with clinical studies in individuals with incident or very mild AD dementia. ${ }^{26,27}$ There is no clear explanation for the increased mortality risk. Risk factors for AD might also be associated with other life-threatening diseases. Alternatively, AD-related cognitive impairments might increase mortality risk because they may hamper diagnosis and management of other diseases or increase the risk of accidents. ${ }^{28,29}$ Increased mortality risk might also have resulted from AD pathological abnormalities, which might compromise the physiological response to other illnesses. ${ }^{30}$ However, further research is needed to understand the relation between preclinical AD and mortality.

The proportion of participants with preclinical AD was higher in older individuals and in APOE $\varepsilon 4$ carriers than in younger individuals and non-carriers, which is in line with findings from previous studies. ${ }^{31}$ However, neither age ( $<72$ years vs $\geq 72$ years) nor APOE genotype predicted rate of decline, although these subanalyses had limited statistical power owing to the small sample sizes. Although APOE $\varepsilon 4$ is often a good predictor of cognitive decline in unselected populations, the absence of its prognostic utility in individuals with $\mathrm{AD}$ pathological abnormalities is consistent with findings from previous studies. ${ }^{32}$

$23 \%$ of participants in this study had SNAP, in line with findings from the MCSA. ${ }^{16}$ Cognitive decline in the SNAP group was similar to that in normal individuals, although there was weak evidence of increased mortality. There were no to low AD pathological changes on autopsy, suggesting that these individuals might have other diseases.

The selection of cutoffs is crucial for categorisation of NIA-AA stages. ${ }^{5}$ We used the Youden index to define the CSF cutoffs. These values were lower than those previously used in a similar cohort $\left(\mathrm{A} \beta_{1-42}<500 \mathrm{pg} / \mathrm{mL}\right.$, t-tau $>440 \mathrm{pg} / \mathrm{mL}$, and p-tau $\left.181>78 \mathrm{pg} / \mathrm{mL}\right) .{ }^{33}$ Use of the previous cutoffs would lead to a slightly higher proportion of preclinical AD (40\%), but the progression to symptomatic AD remained the same (appendix). Our cognitive cutoff at the tenth percentile was in line with that used in the MCSA.6,16

Also, the choice of cognitive tests might affect the NIA-AA staging and outcome. We defined subtle cognitive changes as low scores on a memory composite test. If subtle cognitive change was defined as a low score in any cognitive domain (episodic memory, semantic memory, working memory, or visuospatial score, as described by Johnson and colleagues ${ }^{15}$ ), the number of individuals in stage 3 and the unclassified group would increase. However, progression rates to $\mathrm{CDR}$ at least 0.5 , symptomatic $\mathrm{AD}$ in these groups would be lower (appendix). Although we used a composite score of three memory tests on the basis of factor analyses, the use of a specific memory test could have led to different results.

Participants in stage 3 differed from those with MCI or early dementia in that they had a CDR score of 0 and therefore no change in cognitive function and no interference in activities of daily living. Still, some of these participants might have met psychometric criteria for MCI. Findings from a study in autosomal dominant AD mutation carriers ${ }^{34}$ 
showed that individuals with preclinical AD might have cognitive impairments without disturbance in functional abilities, and thus appear clinically normal.

14 participants remained unclassified and their outcome has not been investigated previously. They had an increased risk of progression to symptomatic AD but not of mortality compared with the normal group (appendix). Although amyloid pathology might be present in these individuals, future studies are needed to clarify their characteristics and outcome.

Our results are consistent with those recently reported in the MCSA,6,16 although there were important differences in study design. The MCSA used imaging markers for staging individuals and cognitive tests to define clinical diagnosis rather than the CDR. Furthermore, the biomarker cutoffs were defined as those yielding $90 \%$ sensitivity for diagnosing AD dementia from a separate AD cohort and a global cognitive test score was used to define subtle cognitive change. The similarity in findings between the studies suggests that CSF and imaging markers might be equally effective for identification of individuals with preclinical $\mathrm{AD}$ and prediction of clinical outcome. However, this suggestion does not imply that CSF and imaging makers are equivalent. Head-to-head comparison might yield a different conclusion.

The major strengths of this study are the large sample size of well-characterised participants and the long follow-up period of up to 15 years (mean 4 years). However, our study has several limitations. Because participants agreed to take part in a longitudinal study, including multiple neuroimaging procedures and serial lumbar punctures, they are unlikely to be representative of the general population; nor were they selected at random from the population. However, our sample is similar to other research samples of cognitively normal older adults and people with early symptomatic AD. ${ }^{35}$ Also, the number of participants who progressed to symptomatic AD in each stage was small and results should therefore be interpreted carefully. Furthermore, AD clinical diagnosis at follow-up was neuropathologically validated in only a small subset of participants. Thus, some participants might have been misclassified, although the rate of confirmation of $A D$ diagnosis post mortem at the KADRC is high (93\%). ${ }^{7}$ Similar to participants in the MCSA, participants in our study were mainly white and highly educated, and findings might not apply to individuals with other backgrounds. Although we included cognitively normal individuals (CDR 0), 18 of them had a CDR-SB score of 0.5 (one score of 0.5 in a non-memory domain) and could be considered suspicious because these people might not be truly unimpaired. However, analyses without these participants revealed similar results (appendix).

Although we regard this study as preliminary and hypothesis generating, our findings have several important implications. First, these findings show that preclinical AD is common and can be diagnosed by CSF markers, as shown by neuropathological validation in eight of nine participants who underwent autopsy. The strong association between preclinical AD and future cognitive decline and mortality makes preclinical AD an important target for therapeutic intervention. Second, they show that the proposed NIA-AA staging of preclinical $A D$ represents different disease stages in view of differences in rate of 
progression. Third, the findings from this study have implications for the design of secondary prevention trials. Screening of individuals for biomarker assessment according to their age and APOE genotype might be useful, and trials could stratify individuals by preclinical AD stage. The rate of cognitive decline was low compared with that in individuals with $\mathrm{MCI}^{36}$ or dementia. ${ }^{37}$ Thus, trials of preclinical AD need large sample sizes or a longer follow-up to identify effects on cognitive outcome measures. Furthermore, mortality should be considered as an endpoint in trials. Fourth, both occurrence and outcome of preclinical $\mathrm{AD}$ are dependent on tests and CSF cutoffs used, which highlights the need for standardisation. 


\section{REFERENCES}

1. Price J, Morris JC. Tangles and plaques in nondemented aging and "preclinical" Alzheimer's disease. Ann Neurol 1999; 45: 358-68.

2. Hulette C, Welsh-Bohmer K, Murray M, Saunders A, Mash D, Mcintyre L. Neuropathological and neuropsychological changes in "normal" aging: evidence for preclinical Alzheimer disease in cognitively normal individuals. J Neuropathol Exp Neurol 1998; 57: 1168-74.

3. Braak H, Braak E. Frequency of stages of Alzheimer-related lesions in different age categories. Neurobiol Aging 1997; 18: 351-57.

4. Bateman RJ, Xiong C, Benzinger TL, et al. Clinical and biomarker changes in dominantly inherited Alzheimer's disease. N Engl J Med 2012; 367: 795-804.

5. Sperling RA, Aisen PS, Beckett LA, et al. Toward defining the preclinical stages of Alzheimer's disease: recommendations from the National Institute on Aging and the Alzheimer's Association workgroup. Alzheimers Dement 2011; 7: 280-92.

6. Knopman DS, Jack CR, Wiste HJ, et al. Short-term clinical outcomes for stages of NIA-AA preclinical Alzheimer disease. Neurology 2012; 78: 1576-82.

7. Berg L, McKeel DW, Miller JP, et al. Clinicopathologic studies in cognitively healthy aging and Alzheimer's disease: relation of histologic markers to dementia severity, age, sex, and ApoE genotype. Arch Neurol 1998; 55: 326-35.

8. Morris JC. The clinical dementia rating (CDR): current version and scoring rules. Neurology 1993; 43: 2412-14.

9. Johnson DK, Storandt M, Morris JC, Langford ZD, Galvin JE. Cognitive profiles in dementia: Alzheimer disease versus nondemented aging. Neurology 2008; 71: 1783-89.

10. Berg L, Miller JP, Baty J, Rubin EH, Morris JC, Figiel G. Mild senile dementia of the Alzheimer type. 4. Evaluation of intervention. Ann Neurol 1992; 31: 242-49.

11. Storandt M, Grant EA, Miller JP, Morris JC. Longitudinal course and neuropathologic outcomes in original vs. revised MCI and in pre-MCI. Neurology 2006; 67: 467-73.

12. Bondi MW, Jak AJ, Delano-Wood L, Jacobson MW, Delis DC, Salmon DP. Neuropsychological contributions to the early identification of Alzheimer's disease. Neuropsychol Rev 2008; 18: 73-90.

13. Grober E, Buschke H, Crystal H, Bang S. Screening for dementia by memory testing. Neurology 1988; 38: 900-03.

14. Wechsler D. Manual: Wechsler memory scale-revised. San Antonio, TX: Psychological Corporation, 1987.

15. Johnson DK, Storandt M, Morris JC, Galvin JE. Longitudinal study of the transition from healthy aging to Alzheimer's disease. Arch Neurol 2009; 66: 1254-59.

16. Jack CR, Knopman DS, Weigand SD, et al. An operational approach to National Institute on AgingAlzheimer's Association criteria for preclinical Alzheimer disease. Ann Neurol 2012; 71: 765-75.

17. McKhann G, Drachman D, Folstein M, Katzman R, Price D, Stadlan EM. Clinical diagnosis of Alzheimer's disease: report of the NINCDS-ADRDA Work-Group under the auspices of the Department of Health and Human Services Task Force on Alzheimer's disease. Neurology 1984; 34: 939-44.

18. Albert MS, DeKosky ST, Dickson D, et al. The diagnosis of mild cognitive impairment due to Alzheimer's disease: recommendations from the National Institute on Aging and Alzheimer's Association workgroup. Alzheimers Dement 2011; 7: 270-79.

19. Dubois B, Feldman HH, Jacova C, et al. Research criteria for the diagnosis of Alzheimer's disease: revising the NINCDS-ADRDA criteria. Lancet Neurol 2007; 6: 734-46. 
20. Cairns NJ, Taylor-Reinwald L, Morris JC, and the Alzheimer's Disease Neuroimaging Initiative. Autopsy consent, brain collection, and standardized neuropathologic assessment of ADNI participants: the essential role of the neuropathology core. Alzheimers Dement 2010; 6: 274-79.

21. Montine TJ, Phelps $\mathrm{CH}$, Beach TG, et al. National Institute on Aging-Alzheimer's Association guidelines for the neuropathologic assessment of Alzheimer's disease: a practical approach. Acta Neuropathol 2012; 123: 1-11.

22. Castellano JM, Kim J, Stewart FR, et al. Human APOE isoforms differentially regulate brain amyloid$\beta$ peptide clearance. Sci Transl Med 2011; 3: 89ra57.

23. Fine J, Gray R. A proportional hazards model for the subdistribution of a competing risk. J Amer Statist Assoc 1999; 94: 496-509.

24. Diggle PJ, Heagerty P, Liang K-Y, Zeger SL. Analysis of longitudinal data, 2nd edn. New York: Oxford University Press, 2002.

25. Akaike H. A new look at the statistical model identifi cation. IEEE Trans Automat Contr 1974; 19: 716-23.

26. L.nnroos E, Kyyr.nen P, Bell JS, van der Cammen TJ, Hartikainen S. Risk of death among persons with Alzheimer's disease: a national register-based nested case-control study. J Alzheimers Dis 2013; 33: 157-64.

27. Wallin AK, Blennow K, Zetterberg H, Londos E, Minthon L, Hansson O. CSF biomarkers predict a more malignant outcome in Alzheimer disease. Neurology 2010; 74: 1531-37.

28. Lopponen M, Raiha I, Isoaho R, Vahlberg T, Puolijoki H, Kivela SL. Dementia associates with undermedication of cardiovascular diseases in the elderly: a population-based study. Dement Geriatr Cogn Disord 2006; 22: 132-41.

29. Wadley VG, Okonkwo O, Crowe M, et al. Mild cognitive impairment and everyday function: an investigation of driving performance. J Geriatr Psychiatry Neurol 2009; 22: 87-94.

30. Simic G, Stanic G, Mladinov M, Jovanov-Milosevic N, Kostovic I, Hof PR. Does Alzheimer's disease begin in the brainstem? Neuropathol Appl Neurobiol 2009; 35: 532-54.

31. Morris JC, Roe CM, Xiong C, et al. APOE predicts amyloid-beta but not tau Alzheimer pathology in cognitively normal aging. Ann Neurol 2010; 67: 122-31.

32. Desikan RS, McEvoy LK, Holland D, et al. Apolipoprotein E $\varepsilon 4$ does not modulate amyloid- $\beta$ associated neurodegeneration in preclinical Alzheimer disease. Am J Neuroradiol 2013; 34: 505-10.

33. Tarawneh R, D’Angelo G, Macy E, et al. Visinin-like protein 1: diagnostic and prognostic biomarker in Alzheimer's disease. Ann Neurol 2011; 70: 274-85.

34. Acosta-Baena N, Sepulveda-Falla D, Lopera-Gomez GM, et al. Pre-dementia clinical stages in presenilin 1 E280A familial early-onset Alzheimer's disease: a retrospective cohort study. Lancet Neurol 2011; 10: 213-20.

35. Villareal DT, Grant E, Miller JP, Strorandt M, McKeel DW, Morris JC. Clinical outcomes of possible versus probable Alzheimer's disease. Neurology 2003; 61: 661-67.

36. Vos S, van Rossum I, Burns L, et al. Test sequence of CSF and MRI biomarkers for prediction of AD in subjects with MCI. Neurobiol Aging 2012; 33: 2272-81.

37. Han L, Cole M, Bellavance F, McCusker J, Primeau F. Tracking cognitive decline in Alzheimer's disease using the mini-mental state examination: a meta-analysis. Int Psychogeriatr 2000; 12: 23147. 


\section{SUPPLEMENTAL DATA}

Supplemental Table 1. Baseline demographics of the independent CDR 0.5 symptomatic AD sample ( $\mathrm{N}=\mathbf{7 4})$

Age

Female, $\mathrm{n}$

Education, y

Race, $\mathrm{n}$

Caucasian

African American

Native Hawaiian and Pacific Islander

$A P O E-\varepsilon 4+, \mathrm{n}$

MMSE

CDR-SB

Episodic memory, z-score

$\mathrm{A} \beta 1-42, \mathrm{pg} / \mathrm{mL}$

T-tau, pg/mL

P-tau181, pg/mL
$74 \cdot 5(5 \cdot 4)$

$36(49 \%)$

$14 \cdot 5(3 \cdot 1)$

$71(96 \%)$

$2(3 \%)$

$1(1 \%)$

$51(69 \%)$

$25 \cdot 9(3 \cdot 0)$

$2 \cdot 5(1 \cdot 0)$

$-1 \cdot 6(1 \cdot 0)$

424 (219)

607 (293)

97 (48)

Results are mean (SD) or number (\%). Episodic memory is a composite score of the Associate Learning Test, Logical Memory Test, and Selective Reminding Test. AD=Alzheimer's Disease, APOE=Apolipoprotein E, MMSE=Mini Mental State Examination (range 0-30, with 30 as the best score), CDR-SB=Clinical Dementia Rating scale Sum of Boxes (range $0-16$, with 0 as the best score), $A \beta=$ beta amyloid, $\mathrm{p}$-tau=phosphorylated tau, $\mathrm{t}$-tau=total tau.

Supplementary Table 2. Overview of concepts for mild cognitive impairments

\begin{tabular}{|c|c|c|c|c|}
\hline \multirow[t]{2}{*}{ Construct } & \multicolumn{4}{|l|}{ Definition } \\
\hline & $\begin{array}{l}\text { Psychometric test } \\
\text { impairment }\end{array}$ & Functional impairment & $\begin{array}{l}\text { Clinical dementia } \\
\text { diagnosis } \\
\text { according to } \\
\text { DSM IV or ICD-10 }\end{array}$ & $\begin{array}{l}\text { Biomarker } \\
\text { status }\end{array}$ \\
\hline $\begin{array}{l}\mathrm{CDR} 0 \cdot 5 \\
\text { symptomatic } \\
\mathrm{AD}^{1}\end{array}$ & $\begin{array}{l}\text { Defined cut-offs } \\
\text { not utilized }\end{array}$ & $\begin{array}{l}\text { Very mild to mild change } \\
\text { in daily functioning in } \\
\text { memory and at least } 1 \\
\text { non-memory domain }\end{array}$ & Some & Not needed \\
\hline $\begin{array}{l}\text { Amnestic } \\
\mathrm{MCI}^{2}\end{array}$ & $\begin{array}{l}\text { Yes, in memory } \\
\text { domain }\end{array}$ & $\begin{array}{l}\text { Subjective report of } \\
\text { cognitive decline }\end{array}$ & No & Not needed \\
\hline $\begin{array}{l}\text { MCI due to } \\
\mathrm{AD}^{3}\end{array}$ & $\begin{array}{l}\text { Yes, in any } \\
\text { cognitive domain }\end{array}$ & $\begin{array}{l}\text { Cognitive concern } \\
\text { reflecting a change in } \\
\text { cognition }\end{array}$ & No & Abnormal \\
\hline $\begin{array}{l}\text { Prodromal } \\
\mathrm{AD}^{4}\end{array}$ & $\begin{array}{l}\text { Yes, in memory } \\
\text { domain }\end{array}$ & $\begin{array}{l}\text { Complaints of memory } \\
\text { decline }\end{array}$ & No & Abnormal \\
\hline
\end{tabular}

References: 1. Morris JC. The Clinical Dementia Rating (CDR): current version and scoring rules. Neurology 1993; 43: 2412-14. 2. Petersen RC. Mild cognitive impairment as a diagnostic entity. Journal of Internal Medicine 2004; 256: 183-94. 3. Albert MS, DeKosky ST, Dickson D, Dubois B, Feldman HH, Fox NC, et al. The diagnosis of mild cognitive impairment due to Alzheimer's disease: Recommendations from the National Institute on Aging and Alzheimer's Association workgroup. Alzheimers Dement 2011; 7: 270-9. 4. Dubois B, Feldman HH, Jacova C, Dekosky ST, Barberger-Gateau P, Cummings J, et al. Research criteria for the diagnosis of Alzheimer's disease: revising the NINCDSADRDA criteria. Lancet Neurol 2007; 6: 734-46. 


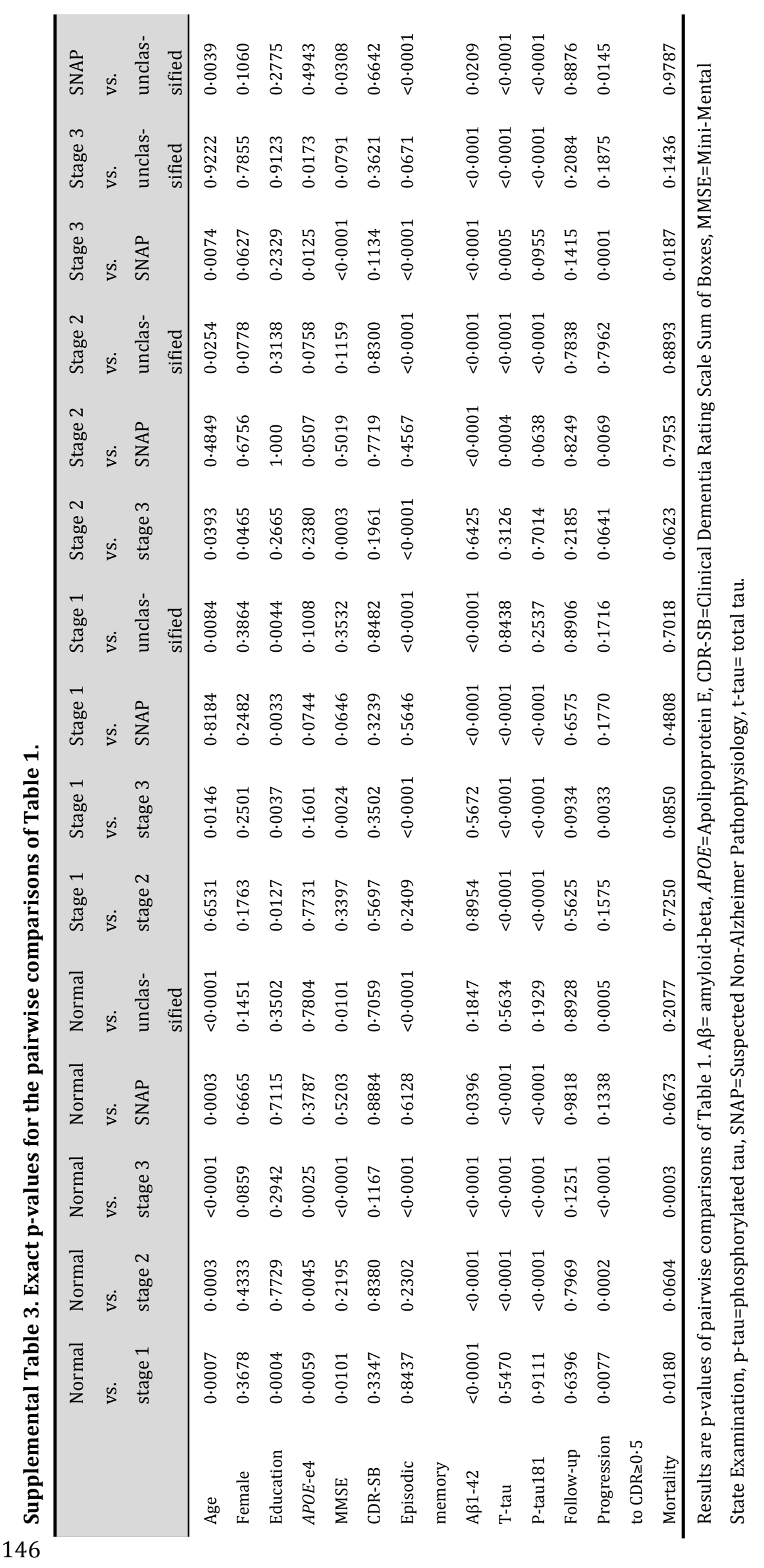


Supplemental Table 4. Distribution of preclinical AD stages

\begin{tabular}{|c|c|c|c|c|c|c|}
\hline Stage & $\begin{array}{l}\text { CSF A } \beta 1-42 \\
<459 \\
\mathrm{pg} / \mathrm{mL}\end{array}$ & $\begin{array}{l}\text { CSF t-tau } \\
>339 \\
\mathrm{pg} / \mathrm{mL}\end{array}$ & $\begin{array}{l}\text { CSF p-tau181 } \\
>67 \mathrm{pg} / \mathrm{mL}\end{array}$ & $\begin{array}{l}\text { Cognition } \\
<-1 \cdot 25 \text { SD }\end{array}$ & $\mathrm{N}(\%)$ & $\begin{array}{l}\text { Overall N } \\
(\%)\end{array}$ \\
\hline $\begin{array}{l}\text { Normal } \\
\text { group }\end{array}$ & - & - & - & - & $\begin{array}{l}129 \\
(41 \cdot 5)\end{array}$ & $129(41 \cdot 5)$ \\
\hline Stage 1 & + & - & - & - & $47(15)$ & $47(15)$ \\
\hline \multirow[t]{3}{*}{ Stage 2} & + & + & - & - & $8(3)$ & $36(12)$ \\
\hline & + & - & + & - & $5(2)$ & \\
\hline & + & + & + & - & $23(7)$ & \\
\hline \multirow[t]{2}{*}{ Stage 3} & + & + & - & + & $1(0 \cdot 5)$ & $13(4)$ \\
\hline & + & + & + & + & $12(3 \cdot 5)$ & \\
\hline \multirow[t]{5}{*}{ SNAP group } & - & + & - & - & $14(4 \cdot 5)$ & $72(23)$ \\
\hline & - & + & - & + & $2(0 \cdot 5)$ & \\
\hline & - & - & + & - & $9(3)$ & \\
\hline & - & + & + & - & $45(14 \cdot 5)$ & \\
\hline & - & + & + & + & $2(0 \cdot 5)$ & \\
\hline \multirow[t]{2}{*}{ Unclassified } & - & - & - & + & $11(3 \cdot 5)$ & $14(4 \cdot 5)$ \\
\hline & + & - & - & + & $3(1)$ & \\
\hline
\end{tabular}

Results are number (\%) of participants identified in the different stages based on CSF markers with optimal Youden cut-offs: Abnormal CSF A $31-42<459$ pg/mL, t-tau >339 pg/mL, p-tau181 >67 pg/mL. Cognition was an episodic memory composite score of the Associate Learning Test, Logical Memory Test, and Selective Reminding Test, with a cut-off at the lowest $10^{\text {th }}$ percentile: $-1 \cdot 25 \mathrm{SD} . \mathrm{A} \beta=$ beta amyloid, $\mathrm{AD}=$ Alzheimer's Disease, $\mathrm{p}$-tau=phosphorylated tau, SNAP=Suspected Non-Alzheimer Pathophysiology, t-tau=total tau. 


\section{Supplemental Table 5. Preclinical AD and its outcome according to age and $A P O E$ genotype}

\begin{tabular}{|c|c|c|c|c|}
\hline \multirow[t]{2}{*}{ A } & \multicolumn{2}{|c|}{ Baseline prevalence } & \multicolumn{2}{|c|}{$\begin{array}{l}\text { Progression to } C D R \geq 0 \cdot 5 \text { symptomatic } \\
A D\end{array}$} \\
\hline & $\begin{array}{l}\text { Age } \leq 72 \\
(n=169)\end{array}$ & $\begin{array}{l}\text { Age }>72 \\
(n=142)\end{array}$ & $\begin{array}{l}\text { Age } \leq 72 \\
(n=6)\end{array}$ & $\begin{array}{l}\text { Age }>72 \\
(n=25)\end{array}$ \\
\hline No preclinical AD & $125(74 \%)$ & $90(64 \cdot 5 \%)^{*}$ & $1(1 \%)$ & $9(10 \%)$ \\
\hline Normal group & $90(53 \cdot 5 \%)$ & $39(27 \cdot 5 \%)$ & $0(0 \%)$ & $2(5 \%)$ \\
\hline SNAP group & $31(18 \cdot 5 \%)$ & $41(29 \%)$ & $1(3 \%)$ & $3(7 \%)$ \\
\hline Unclassified & $4(2 \%)$ & $10(7 \%)$ & $0(0 \%)$ & $4(40 \%)$ \\
\hline Preclinical AD & $44(26 \%)$ & $52(36 \cdot 5 \%)$ & $5(11 \%)$ & $17(33 \%)$ \\
\hline Stage 1 & $25(15 \%)$ & $22(15 \cdot 5 \%)$ & $1(4 \%)$ & $5(23 \%)$ \\
\hline Stage 2 & $17(10 \%)$ & $19(13 \%)$ & $2(12 \%)$ & $7(37 \%)$ \\
\hline Stage 3 & $2(1 \%)$ & $11(8 \%)$ & $2(100 \%)$ & $5(45 \cdot 5 \%)$ \\
\hline \multirow[t]{2}{*}{ B } & \multicolumn{2}{|c|}{ Baseline prevalence } & \multicolumn{2}{|c|}{$\begin{array}{l}\text { Progression to } \mathrm{CDR} \geq 0 \cdot 5 \text { symptomatic } \\
\mathrm{AD}\end{array}$} \\
\hline & $\begin{array}{l}A P O E-\varepsilon 4- \\
(\mathrm{n}=205)\end{array}$ & $\begin{array}{l}A P O E-\varepsilon 4+ \\
(\mathrm{n}=106)\end{array}$ & $\begin{array}{l}A P O E-\varepsilon 4- \\
(\mathrm{n}=18)\end{array}$ & $\begin{array}{l}A P O E-\varepsilon 4+ \\
(\mathrm{n}=13)\end{array}$ \\
\hline No preclinical AD & $158(77 \%)$ & $57(53 \%)^{* * *}$ & $7(4 \%)$ & $3(5 \%)$ \\
\hline Normal group & $97(47 \%)$ & $32(30 \%)$ & $2(2 \%)$ & $0(0 \%)$ \\
\hline SNAP group & $50(24 \cdot 5 \%)$ & $22(21 \%)$ & $2(4 \%)$ & $2(9 \%)$ \\
\hline Unclassified & $11(5 \cdot 5 \%)$ & $3(2 \%)$ & $3(27 \%)$ & $1(33 \%)$ \\
\hline Preclinical AD & $47(23 \%)$ & $49(47 \%)$ & $11(23 \%)$ & $11(22 \%)$ \\
\hline Stage 1 & $25(12 \%)$ & $22(21 \%)$ & $2(8 \%)$ & $4(18 \%)$ \\
\hline Stage 2 & $18(9 \%)$ & $18(17 \%)$ & $6(33 \%)$ & $3(17 \%)$ \\
\hline Stage 3 & $4(2 \%)$ & $9(9 \%)$ & $3(75 \%)$ & $4(44 \%)$ \\
\hline
\end{tabular}

Data are baseline number (\%) of participants with and without preclinical AD, and number (\%) of these participants that progressed to $\mathrm{CDR} \geq 0 \cdot 5$ symptomatic $\mathrm{AD}$ by dichotomous age (A) and $A P O E$ genotype (B) grouping. $\mathrm{AD}=$ Alzheimer's Disease, $A P O E=$ Apolipoprotein E, CDR=Clinical Dementia Rating scale, SNAP=Suspected NonAlzheimer Pathophysiology. ${ }^{*} \mathrm{P}<0 \cdot 05$ compared to age $\leq 72,{ }^{* * *} \mathrm{p}<0 \cdot 001$ compared to APOE-e4-, based on Chi-squared tests for 2 by 2 tables. 
A

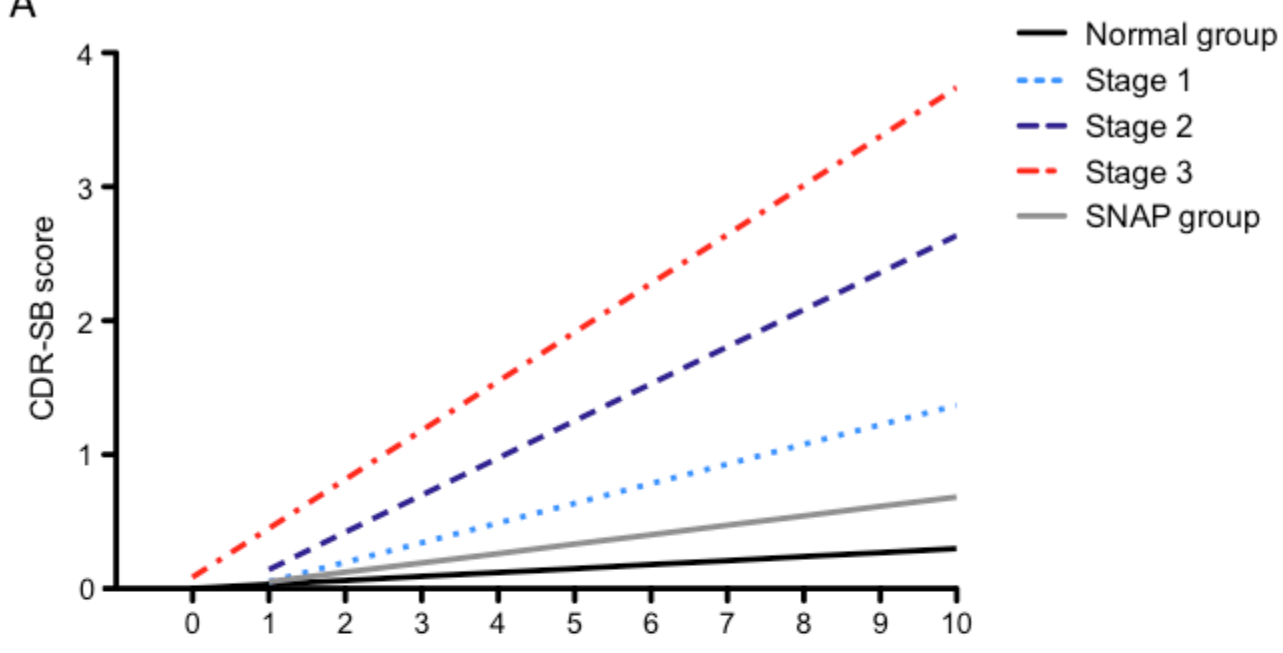

B

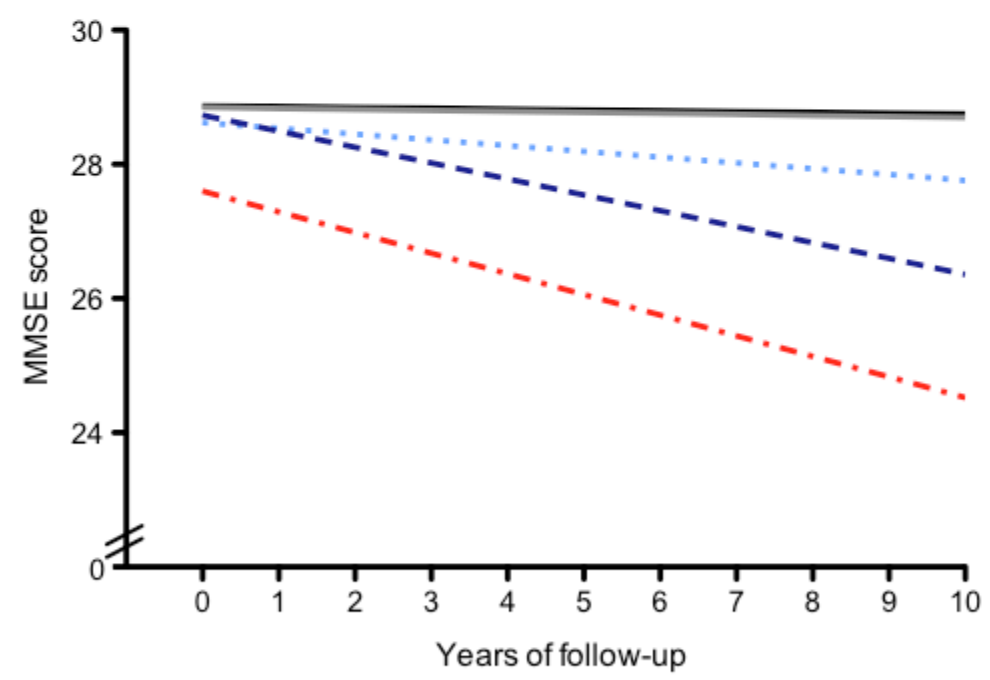

\section{Supplemental Figure 1. Annual rate of change in CDR-SB and MMSE by preclinical AD stage}

Graphs show the estimated annual rate of change in CDR-SB (A) and MMSE (B), based on slopes according to each preclinical $\mathrm{AD}$ stage, corrected for age, gender, education, and $A P O E$ genotype. The black line represents participants in the normal group; light blue, stage 1; dark blue, stage 2; red, stage 3; and grey, SNAP. CDR-SB=Clinical Dementia Rating scale Sum of Boxes (range 0-18, with 0 as the best score), MMSE=Mini Mental State Examination (range 0-30, with 30 as the best score). 
CHAPTER 8

A

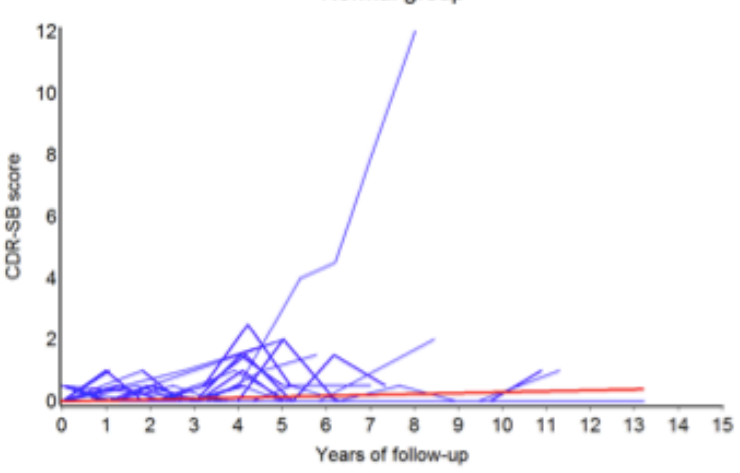

Stage 2

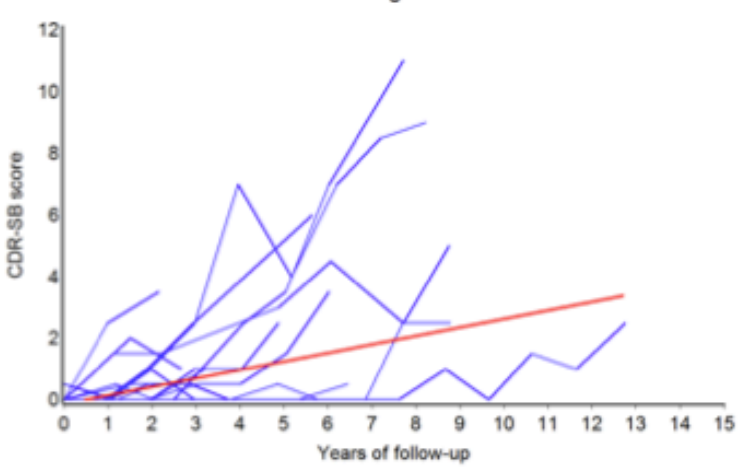

SNAP group

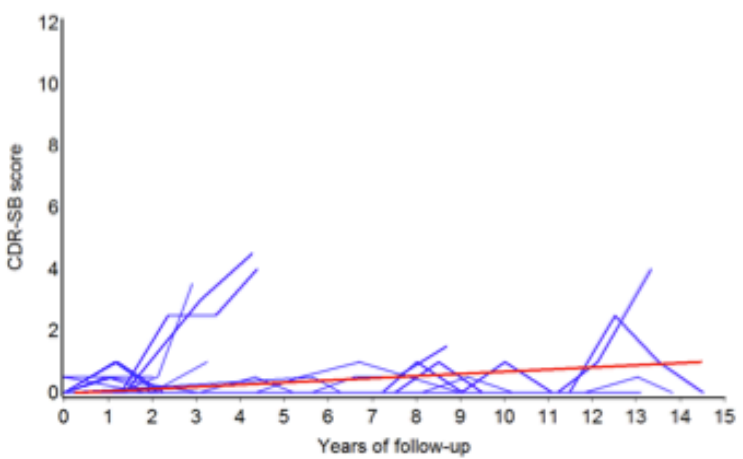

Stage 1

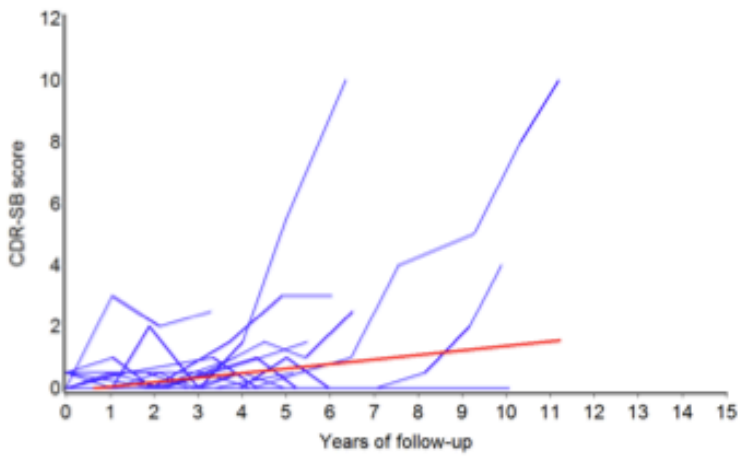

Stage 3

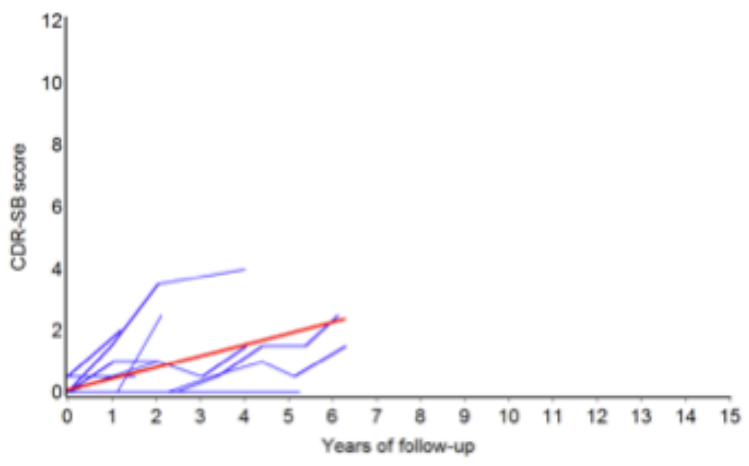

150 

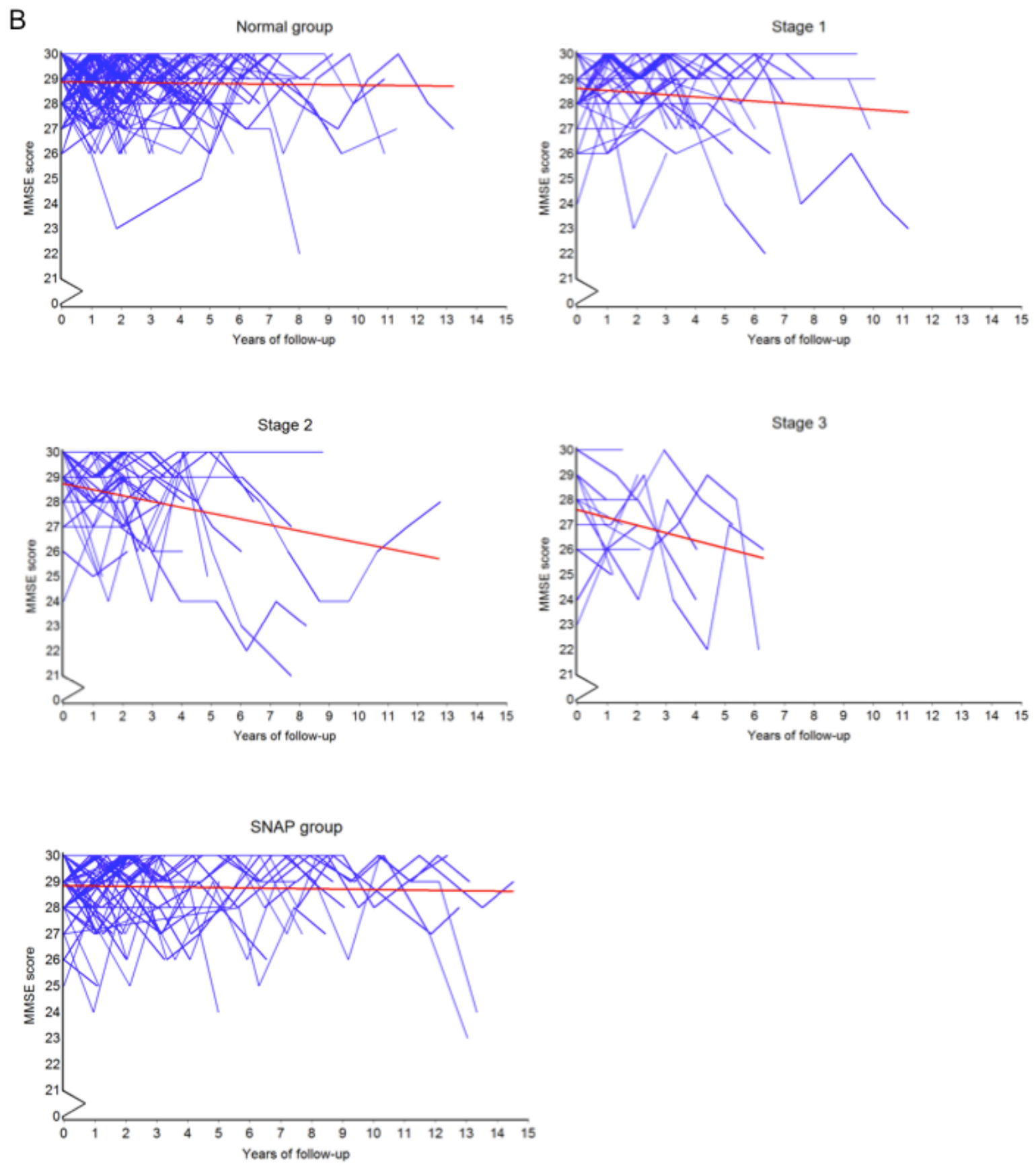

\section{Supplemental Figure 2. Individual cognitive trajectories for CDR-SB and MMSE by preclinical AD stage}

Graphs show individual cognitive trajectories (blue) and the overall estimated annual rate of change (red) for CDR-SB (A) and MMSE (B) by preclinical AD stage. CDR-SB=Clinical Dementia Rating scale Sum of Boxes (range 0-18, with 0 as the best score), MMSE=Mini Mental State Examination (range 0-30, with 30 as the best score), SNAP= Suspected NonAlzheimer Pathophysiology. 


\section{Supplemental Text 1. Cohort information}

The overall rate of progression from CDR 0 to CDR 0.5 or greater in our sample was $10 \%$ $(32 / 311)$ over an average of 4 years of follow-up. This percent is comparable to the Mayo Clinic Study of Aging (MCSA), a population based longitudinal program in which many participants have multiple imaging procedures and some have lumbar puncture. The overall rate of progression from cognitive normality to MCI in the MCSA sample was $20 \%$ (296/1450) with a median follow-up of 3.4 years, but the MCI construct in the MCSA program was unstable as $34 \%$ of $\mathrm{MCI}$ individuals reverted to cognitive normality at subsequent follow-up. ${ }^{1}$ Removing the MCSA individuals who later reverted to normal leaves 196 of the $1450(13 \cdot 5 \%)$ cognitively normal persons who developed MCI/symptomatic AD, very similar to our $10 \%$.

\section{Reference}

1. Roberts RO, Geda YE, Knopman DS, et al. The incidence of MCI differs by subtype and is higher in men: the Mayo Clinic Study of Aging. Neurology 2012; 78: 342-51. 


\section{Supplemental Table 6. Preclinical AD and its outcome using different classification approaches}

A) Use of different CSF cutoffs; $\mathrm{A} \beta 1-42<500$, t-tau $<440$, p-tau181 $<78 \mathrm{pg} / \mathrm{mL}$

\begin{tabular}{lllllll}
\hline & $\begin{array}{l}\text { Normal } \\
\text { group }\end{array}$ & Stage 1 & Stage 2 & Stage 3 & $\begin{array}{l}\text { SNAP } \\
\text { group }\end{array}$ & $\begin{array}{l}\text { Unclassified } \\
\text { group }\end{array}$ \\
\hline $\begin{array}{l}\text { Proportion stages, n } \\
\begin{array}{l}\text { Progression to } \\
\text { CDR } \geq 0 \cdot 5\end{array}\end{array}$ & $131(42 \%)$ & $85(27 \%)$ & $28(9 \%)$ & $13(4 \%)$ & $37(12 \%)$ & $17(6 \%)$ \\
$\begin{array}{l}\text { symptomatic AD, } \mathrm{n} \\
\text { Mortality, } \mathrm{n}\end{array}$ & & $9(11 \%)$ & $7(25 \%)$ & $7(54 \%)$ & $2(5 \%)$ & $5(29 \%)$ \\
\hline
\end{tabular}

B) Stage 3 defined as a score in the lowest $10^{\text {th }}$ percentile of any cognitive domain

\begin{tabular}{|c|c|c|c|c|c|c|}
\hline & $\begin{array}{l}\text { Normal } \\
\text { group }\end{array}$ & Stage 1 & Stage 2 & Stage 3 & $\begin{array}{l}\text { SNAP } \\
\text { group }\end{array}$ & $\begin{array}{l}\text { Unclassified } \\
\text { group }\end{array}$ \\
\hline Proportion stages, $\mathrm{n}$ & $104(33 \%)$ & $37(12 \%)$ & $28(9 \%)$ & $21(7 \%)$ & $71(23 \%)$ & $50(16 \%)$ \\
\hline $\begin{array}{l}\text { Progression to } \\
\mathrm{CDR} \geq 0 \cdot 5 \\
\text { symptomatic } \mathrm{AD}, \mathrm{n}\end{array}$ & $2(2 \%)$ & $6(16 \%)$ & $8(29 \%)$ & $8(38 \%)$ & $4(6 \%)$ & $4(8 \%)$ \\
\hline Mortality, n & $0(0 \%)$ & $5(14 \%)$ & $1(4 \%)$ & $6(29 \%)$ & $4(6 \%)$ & $4(8 \%)$ \\
\hline
\end{tabular}

C) Participants with a baseline CDR-SB of $0 \cdot 5$ excluded ( $\mathrm{n}=293)$

\begin{tabular}{lllllll}
\hline & $\begin{array}{l}\text { Normal } \\
\text { group }\end{array}$ & Stage 1 & Stage 2 & Stage 3 & $\begin{array}{l}\text { SNAP } \\
\text { group }\end{array}$ & $\begin{array}{l}\text { Unclassified } \\
\text { group }\end{array}$ \\
\hline Proportion stages, n & $123(42 \%)$ & $43(15 \%)$ & $34(12 \%)$ & $11(4 \%)$ & $69(24 \%)$ & $13(4 \%)$ \\
$\begin{array}{l}\text { Progression to } \\
\text { CDR } \geq 0 \cdot 5\end{array}$ & $2(2 \%)$ & $6(14 \%)$ & $9(27 \%)$ & $6(55 \%)$ & $4(6 \%)$ & $3(23 \%)$ \\
$\begin{array}{l}\text { symptomatic AD, n } \\
\text { Mortality, n }\end{array}$ & $2(2 \%)$ & $5(12 \%)$ & $2(6 \%)$ & $4(36 \%)$ & $4(6 \%)$ & $0(0 \%)$ \\
\hline
\end{tabular}

Results are number (\%) for (A) previously applied CSF cut-offs of our center, (B) a different definition of stage 3 based on the episodic memory, semantic memory, working memory, and visuospatial composite score (described in Johnson

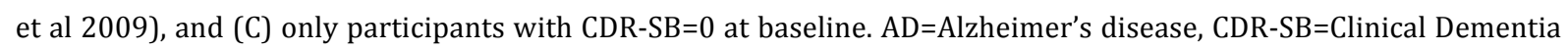
Rating Scale Sum of Boxes, CSF=cerebrospinal fluid, SNAP= Suspected Non-Alzheimer Pathophysiology. 


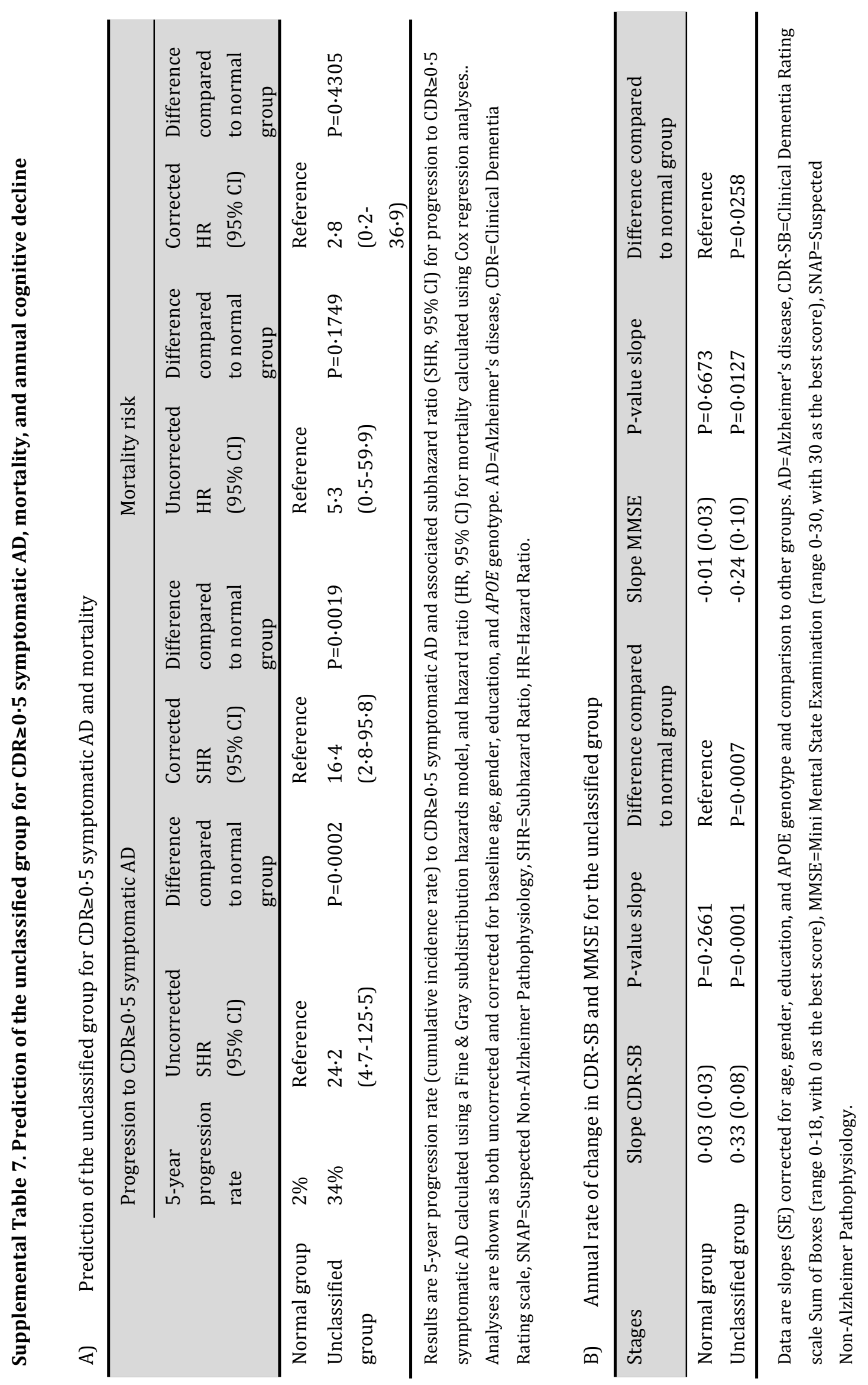




\section{CHAPTER 9}

PREVALENCE AND PROGNOSIS OF PRODROMAL ALZHEIMER'S DISEASE:

A COMPARISON OF THE INTERNATIONAL WORKING GROUP CRITERIA AND NATIONAL INSTITUTE OF AGING-ALZHEIMER'S ASSOCIATION

CRITERIA

Stephanie J.B. Vos, Frans Verhey, Andy Simmons, Michaela Litchmore Dunbar, Giovanni Frisoni, Cristina Muscio, Hilkka Soininen, Sanna-Kaisa Herukka, Erik Salmon, Christine Bastin, Sebastiaan Engelborghs, Stefan Van der Mussele, Isabel Santana, Raquel Lemos, Alexandre Mendonça, Dina Silva, Flavio Nobili, Silvia Morbelli, Robert Perneczky, Mira Didic, Bart van Berckel, Lars-Olof Wahlund, Harald Hampel, Magda Tsolaki, Lennart Minthon, Philip Scheltens, Pieter Jelle Visser and the Alzheimer's Disease Neuroimaging Initiative 


\section{ABSTRACT}

\section{Objective}

Our aim was to compare the International Working Group (IWG) criteria and the National Institute of Aging-Alzheimer Association (NIA-AA) criteria for prodromal AD.

\section{Methods}

1243 subjects were recruited from the EADC network and ADNI. Inclusion criteria were baseline MCI diagnosis and data of at least one AD biomarker (CSF A $\beta$, CSF tau, FDG-PET or MRI) and one clinical follow-up available. 494 (40\%) subjects had both an amyloid and neural injury marker.

\section{Results}

According to the IWG criteria, 670 (54\%) subjects were classified as having prodromal AD. They had at least one abnormal biomarker and impairment in episodic memory. 353 (53\%) of these subjects progressed to AD-type dementia compared to $128(22 \%)$ subjects in the normal group. According to the NIA-AA criteria, 74 (15\%) were classified in the low-ADlikelihood group (no amyloid deposition or neural injury), 250 (51\%) in the high-ADlikelihood group (amyloid deposition and neural injury), 29 (6\%) in the conflicting biomarker group with amyloid deposition without neural injury, and 141 (29\%) in the Suspected Non-Alzheimer Pathology group (SNAP; neural injury without amyloid deposition). At follow-up, 10 (14\%) subjects in the low-AD-likelihood group, 155 (62\%) in the high-AD-likelihood group, 7 (24\%) in the group with amyloid deposition and neural injury, and $42(30 \%)$ in the SNAP group progressed to AD-type dementia.

\section{Conclusions}

Our findings support the use of biomarkers in subjects with MCI according to the IWG and NIA-AA criteria and highlight the importance of differentiating between amyloid and neural injury markers to give an optimal prognosis. 


\section{INTRODUCTION}

Mild cognitive impairment (MCI) usually represents the stage between normal cognitive functioning and dementia and often Alzheimer's disease (AD)-type dementia. ${ }^{1}$ However, not all subjects with $\mathrm{MCI}$ have $\mathrm{AD}$ pathology. ${ }^{2,3}$ Increasing knowledge gained on the underlying pathophysiology of $\mathrm{AD}$ in the last years has led to the development of two sets of research criteria for prodromal AD: those by the International Working Group (IWG) 4, $^{5}$ and those by the National Institute of Aging and the Alzheimer Association (NIA-AA). ${ }^{6}$

These new research criteria allow detection of $\mathrm{AD}$ before the dementia stage and assume that $\mathrm{AD}$ pathophysiological changes in the MCI stage increase the confidence that these subjects will progress to AD-type dementia. The IWG criteria require memory impairment and at least one abnormal AD biomarker for prodromal AD, whereas the NIAAA criteria did not specify cognitive impairment and related the number of positive biomarkers to the likelihood that the diagnosis of $\mathrm{MCI}$ is due to AD. Recent studies showed that these criteria have a fair to good predictive ability for the progression to AD-type dementia. $^{7-9}$ However, it is unknown how these sets of criteria differ in identification of prodromal $\mathrm{AD}$ and in prediction of $\mathrm{AD}$-type dementia.

The aim of the present study was to compare the prevalence and prognostic value of these two sets of diagnostic criteria in subjects with MCI by means of a large multicenter sample and well-established AD biomarkers.

\section{METHODS}

\section{Subjects}

Subjects were recruited from several independent multicenter studies: the DESCRIPA study, ${ }^{10}$ AddNeuroMed study, ${ }^{11}$ the European Alzheimer's Disease Consortium (EADC) PET project, ${ }^{12}$ and the American Alzheimer's Disease Neuroimaging Initiative (ADNI) study ${ }^{13}$ (downloaded in February 2012); and from 7 cohorts of the EADC (Amsterdam, Antwerp, Brescia, Coimbra, Kuopio, Liege, Lisbon). All studies are described more in detail elsewhere. Inclusion criteria of the present study were baseline diagnosis of MCI, availability of at least one of the following biomarker data at baseline: beta amyloid (A $\beta) 1-42$ in cerebrospinal fluid (CSF), tau in CSF, qualitative or quantitative measures of medial temporal lobe atrophy (MTA) or hippocampal volume (HCV) on magnetic resonance imaging (MRI), or cerebral glucose metabolism on brain 18F-fluorodeoxyglucose positron emission tomography (18F FDG PET), and at least one clinical follow-up assessment. Exclusion criteria were diagnosis of dementia at baseline or any other somatic, psychiatric or neurological disorder that might have caused the cognitive complaints or impairments.

\section{Clinical and cognitive assessment}

Clinical and cognitive assessments were performed according to the routine protocol at each site, including a clinical interview, scoring on the MMSE, neuropsychological assessment and biomarker assessment. 
Baseline diagnosis of MCI was made according to the criteria of Petersen et al. ${ }^{1}$ Raw scores on neuropsychological tests were converted to z scores or equivalent scores at each center. Cognitive impairment was defined as z score $<-1.5$ SD or equivalent score on at least one cognitive test. Subjects with a z score $<-1.5$ SD on a memory test were classified as having amnestic MCI. Supplementary Table 1 describes the neuropsychological tests that were used at each center. Decline in cognition was calculated as the difference between the MMSE score at baseline and the score at last follow-up. Primary outcome measures were prevalence of prodromal $\mathrm{AD}$ and progression to AD-type dementia according to the DSMIV $^{14}$ and NINCDS-ADRDA ${ }^{15}$ criteria. Secondary outcome measure was cognitive decline on the MMSE. The study was approved by the Medical Ethics Committee of each center and all the recruited subjects provided written informed consent.

\section{Biomarker assessment}

Biomarker assessment was performed according to the routine protocol at each site. We used routine biomarker cut-offs of each center to define abnormal biomarkers. All biomarker cut-offs are listed in Supplemental Table 2.

\section{Classification according to the IWG and NIA-AA criteria}

For the IWG criteria, subjects were classified in 2 groups. Subjects were classified in the prodromal AD group if they had memory impairment and at least one abnormal biomarker. They were classified in the normal group if all biomarkers were normal or if they had no memory impairment.

For the NIA-AA criteria, we differentiated between amyloid (e.g. CSF A $\beta 1-42$ ) and neuronal injury markers (e.g. CSF tau, FDG-PET, MTA or HCV) for classification. Of the subjects who had an amyloid and neuronal injury marker, subjects were classified in the low-AD-likelihood group if both amyloid and neuronal injury markers were normal, in the high-AD-likelihood group if both amyloid and at least one neuronal injury marker were abnormal, in the conflicting biomarker Isolated Amyloid Pathology (IAP) group if the amyloid marker was abnormal and injury marker normal, and in the conflicting biomarker Suspected Non-Alzheimer Pathophysiology (SNAP) group if the injury marker was abnormal and amyloid marker normal.9, 16 of the subjects who had only one biomarker available, subjects were classified in the intermediate-AD-likelihood group if the marker that was tested was abnormal. Biomarker info was considered uninformative/inconclusive if only one of them was tested and normal.

\section{Statistical analyses}

Statistical analyses were done with SPSS version 19.0 (Chicago, IL, USA) with significance set at $\mathrm{p}<0.05$. Baseline differences between the biomarker subgroups were analyzed using ANOVA for continuous variables and $\chi^{2}$ tests or logistic regression models for categorical variables. Standard Cox proportional hazards models were used to test the predictive ability of the IWG and NIA-AA criteria for AD-type dementia. Analyses were corrected for age, 
gender, and center. The relation between biomarker groups of the IWG and NIA-AA criteria and change on the MMSE was assessed by slope analyses with general linear mixed models. The analyses included the baseline score and last follow-up score (up to 9 years after baseline). The model was specified with a random intercept and slope and with center as a random effect because this model provided the best -2 log likelihood compared with models with simpler covariance structures. Analyses were corrected for age and gender.

\section{RESULTS}

\section{Sample demographics}

We included 1243 subjects. 494 subjects had both an amyloid and neuronal injury marker (CSF A 1-42 and tau/MRI) and 749 subjects had only a neuronal injury marker (FDG-PET $\mathrm{n}=192$; MRI $\mathrm{n}=557$ ). Supplemental Table 3 shows the number of subjects for each biomarker by center and Supplemental Table 4 presents baseline sample characteristics for the total sample and the separate biomarker samples.

\section{IWG criteria}

Of the 1243 subjects, 670 (54\%) were classified as having prodromal AD according to the IWG criteria. Differences in baseline demographics and outcome between the prodromal AD group and the normal group are presented in Table 1. Subjects with prodromal AD were more likely to progress to AD-type dementia than subjects in the normal group with normal biomarkers (HR=3.7, p<0.001; Figure 1, Table 2). Subjects with prodromal AD also showed a larger decline on the MMSE than subjects in the normal group ( $\mathrm{p}<0.001$; Figure 2, Table 3 ). Of the subjects in the normal group with abnormal biomarkers ( $n=163), 52(32 \%)$ progressed to AD-type dementia compared to 75 (18\%) of the subjects in the normal group with normal biomarkers ( $\mathrm{n}=410 ; \mathrm{HR}=1.9, \mathrm{p}=0.001)$.

\section{NIA-AA criteria}

Of the subjects with both an amyloid and neuronal injury marker available ( $n=494), 74$ $(15 \%)$ were classified in the low-AD-likelihood group according to the NIA-AA criteria, 250 (51\%) in the high-AD-likelihood group, 29 (6\%) in the conflicting IAP group, and 141 (29\%) in the conflicting SNAP group. Of the subjects with only a neuronal injury marker available ( $n=749), 413$ (55\%) were classified in the intermediate-AD-likelihood group, and 336 $(45 \%)$ in the inconclusive group. Baseline demographics and outcome of the biomarker groups are presented in Table 1. The high-AD-likelihood, intermediate-AD-likelihood, and SNAP group had a higher risk of progression to AD-type dementia compared to the low-ADlikelihood group (high HR=7.8, $\mathrm{p}<0.001$; intermediate HR=6.4, $\mathrm{p}<0.001$; SNAP HR=2.5, $\mathrm{p}=0.02$; Figure 1, Table 2). The IAP group tended to have a higher risk of progression to ADtype dementia than the low-AD-likelihood group ( $\mathrm{HR}=2.3, \mathrm{p}=0.10)$. Decline on the MMSE showed a similar pattern for the biomarker groups (Figure 2, Table 3). 


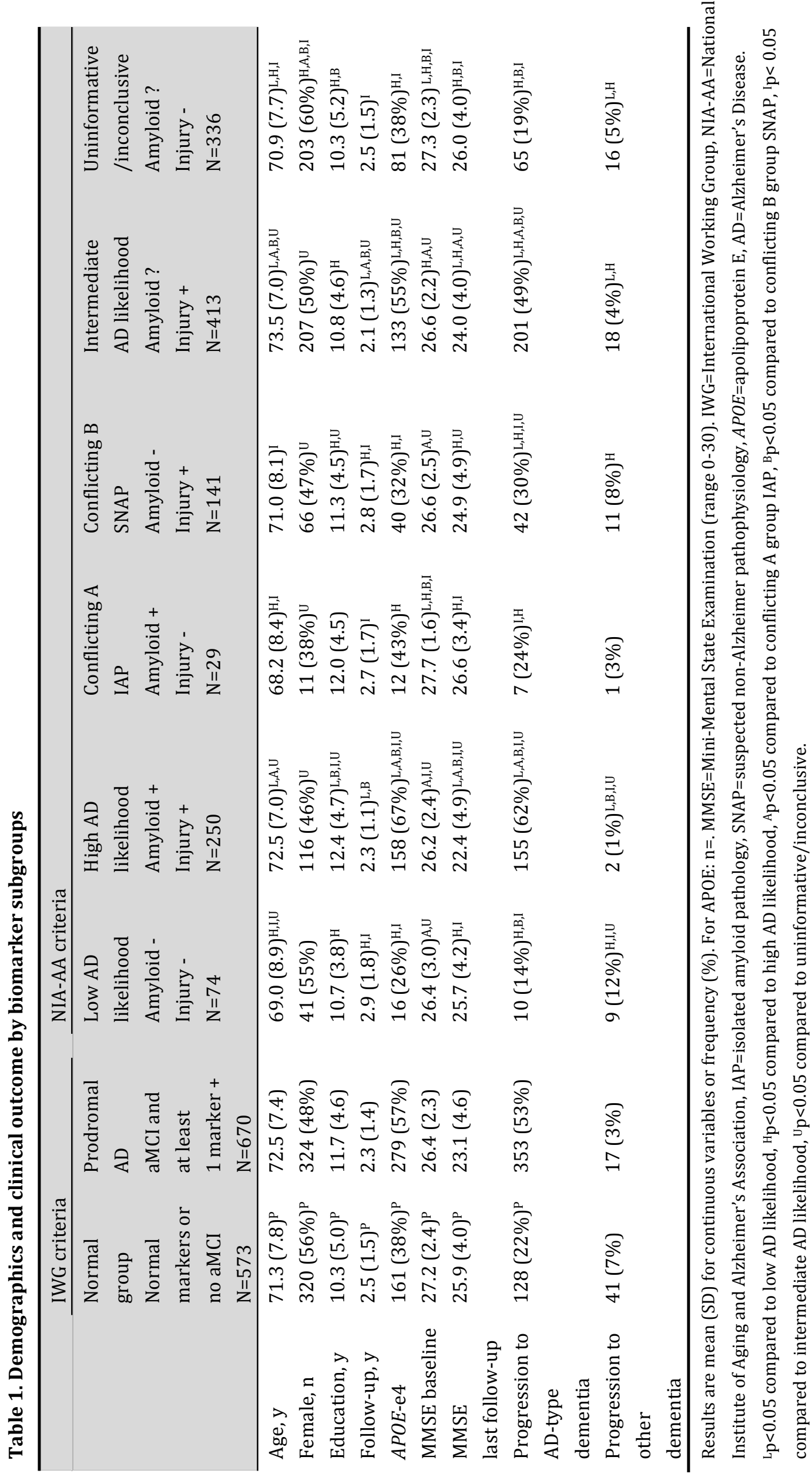



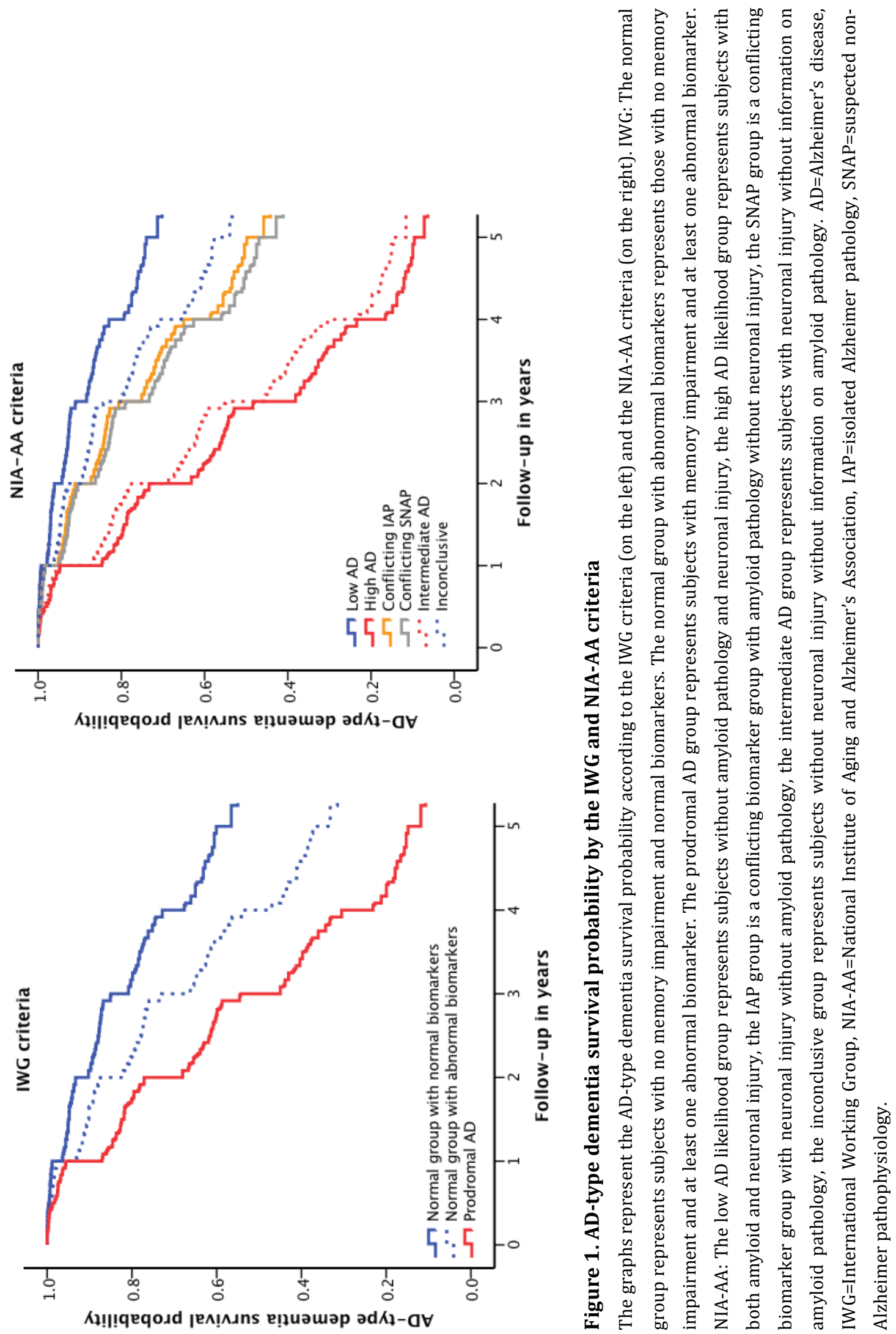
Table 2. AD-type dementia survival probability by the IWG and NIA-AA criteria

\begin{tabular}{lll}
\hline & Hazard Ratio (95\% CI) & P-value \\
\hline IWG & & \\
Normal group with normal biomarkers & Reference & \\
Normal group with abnormal biomarker(s) & $1.9(1.3-2.8)$ & 0.001 \\
Prodromal AD & $3.7(2.9-4.9)$ & $<0.001$ \\
NIA-AA & & \\
Low AD likelihood & Reference & \\
High AD likelihood & $7.8(3.7-16.3)$ & $<0.001$ \\
Conflicting IAP & $2.3(0.8-6.3)$ & 0.10 \\
Conflicting SNAP & $2.5(1.2-5.2)$ & 0.02 \\
Intermediate AD likelihood & $6.4(3.0-13.5)$ & $<0.001$ \\
Uninformative/inconclusive & $1.8(0.8-4.0)$ & 0.13 \\
\hline
\end{tabular}

Results are Hazard Ratios (95\% CI) for progression to AD-type dementia calculated using Cox regression analyses and corrected for age, gender, and center for all the biomarker groups. IWG=International Working Group, NIA$\mathrm{AA}=$ National Institute of Aging and Alzheimer's Association, IAP=isolated amyloid pathology, SNAP=suspected nonAlzheimer pathophysiology, $\mathrm{AD}=$ Alzheimer's Disease.

\section{NIA-AA subanalyses}

The NIA-AA criteria do not differentiate between the several neuronal injury markers. We tested whether the use of different injury markers resulted in different findings. When MRI and CSF tau were used separately in combination with CSF A $\beta 1-42$, results were generally the same (Supplemental Table 5). However, analyses based on CSF tau showed higher progression rates for the SNAP group and lower progression rates for the high-ADlikelihood group compared to the analyses based on MRI (SNAP: 36 vs. 28\%, high: 64 vs. $71 \%)$.

In the subgroup of subjects who had an amyloid marker (CSF A $31-42$ ) and two injury markers (CSF tau and MRI; $n=408$ ), we found that an abnormal amyloid marker in combination with two abnormal injury markers was better predictive for progression to ADtype dementia than an abnormal amyloid marker in combination with only one abnormal injury marker (74\% vs. 41-44\%; Supplemental Table 6). Subjects with conflicting injury markers in combination with amyloid deposition had similar progression rates (41 vs. 44\%; Supplemental Table 6).

The conflicting IAP and SNAP groups unexpectedly showed similar progression rates to AD-type dementia. We compared CSF A 1-42 levels subjects with SNAP who progressed to AD-type dementia with those who did not progress. For ELISA analyses $(n=116)$, we found that SNAP progressors had lower CSF A $\beta 1-42$ levels compared to non-progressors (607 [95\% CI 574-641] vs. 847 [95\% CI 791-904] pg/mL, p<0.001). For xMAP analyses (ADNI, $n=35$ ), we did not find a statistically significant difference between the progressors and non-progressors although this could be a power issue (231 [95\% CI 202-260] vs. 248 [95\% CI 237-259] pg/mL, p=0.22). 


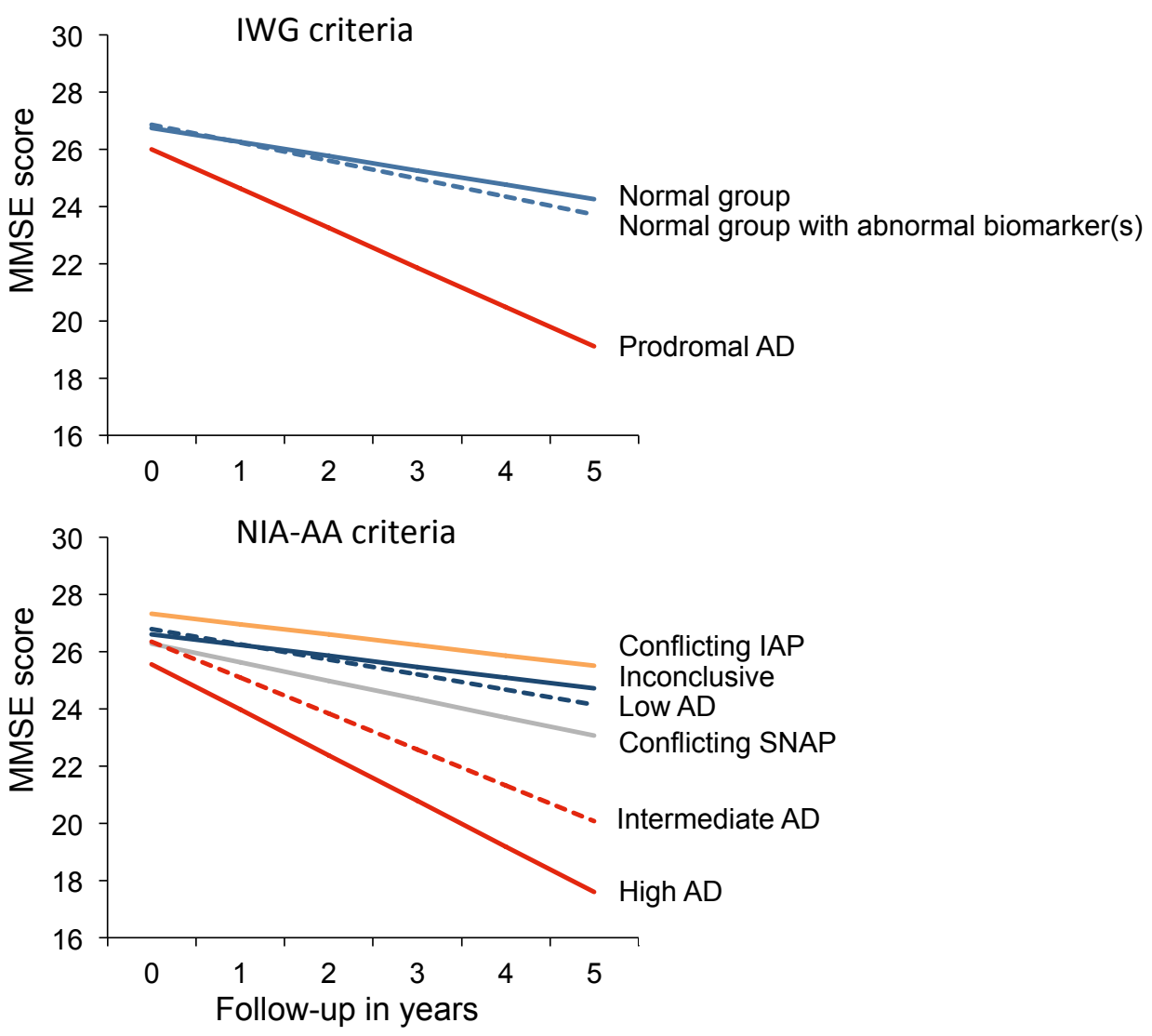

Figure 2. Annual MMSE change for each biomarker group by the IWG and NIA-AA criteria IWG: The normal group represents subjects with no memory impairment and normal biomarkers. The normal group with abnormal biomarkers represents those with no memory impairment and at least one abnormal biomarker. The prodromal AD group represents subjects with memory impairment and at least one abnormal biomarker NIA-AA: The low AD likelihood group represents subjects without amyloid pathology and neuronal injury, the high AD likelihood group represents subjects with both amyloid and neuronal injury, the IAP group is a conflicting biomarker group with amyloid pathology without neuronal injury, the SNAP group is a conflicting biomarker group with neuronal injury without amyloid pathology, the intermediate AD group represents subjects with neuronal injury without information on amyloid pathology, the inconclusive group represents subjects without neuronal injury without information on amyloid pathology. $\mathrm{AD}=$ Alzheimer's disease, $\mathrm{IWG=International} \mathrm{Working} \mathrm{Group,} \mathrm{NIA-AA=National} \mathrm{Institute} \mathrm{of} \mathrm{Aging}$ and Alzheimer's Association, IAP=isolated amyloid pathology, SNAP=suspected non-Alzheimer pathophysiology. 
Table 3. Annual MMSE change for each biomarker group by the IWG and NIA-AA criteria

\begin{tabular}{lll}
\hline & Slope MMSE & P-value slope \\
\hline IWG criteria & & \\
Normal group with normal biomarkers & $-0.50(0.10)$ & $\mathrm{P}<0.001$ \\
Normal group with abnormal biomarkers & $-0.63(0.16)$ & $\mathrm{P}<0.001$ \\
Prodromal AD & $-1.38(0.08)^{* *}$ & $\mathrm{P}<0.001$ \\
& & \\
NIA-AA criteria & & \\
Low AD & $-0.38(0.23)$ & $\mathrm{P}=0.10$ \\
High AD & $-1.59(0.13)^{* *}$ & $\mathrm{P}<0.001$ \\
Conflicting IAP & $-0.36(0.39)$ & $\mathrm{P}=0.35$ \\
Conflicting SNAP & $-0.64(0.17)$ & $\mathrm{P}<0.001$ \\
Intermediate AD & $-1.25(0.12)^{*}$ & $\mathrm{P}<0.001$ \\
Inconclusive & $-0.53(0.11)$ & $\mathrm{P}<0.001$ \\
\hline
\end{tabular}

${ }^{*} \mathrm{P}<0.05$ compared to the normal group/low $\mathrm{AD},{ }^{* *} \mathrm{p}<0.001$ compared to the normal group with normal biomarkers/low $\mathrm{AD}$. AD=Alzheimer's disease, IAP=isolated amyloid pathology, SNAP=suspected non-Alzheimer pathophysiology.

\section{DISCUSSION}

This study is the first large-scale study to compare the recently published IWG and NIA-AA criteria for MCI. Our findings showed that subject classifications based on the IWG and NIAAA criteria were good predictors of likelihood of future cognitive decline and progression to AD-type dementia in subjects with MCI.

The IWG criteria required one abnormal biomarker and episodic memory impairment for classification of prodromal $\mathrm{AD}$. We found that these subjects were 3 times more likely to develop AD-type dementia compared to those classified as being normal. One of the drawbacks of the IWG classification is that the normal group also includes subjects with abnormal biomarkers with non-memory impairment, as they may progress to AD-type dementia. ${ }^{17}$ We found that the use of any impairment instead of memory impairment to define prodromal AD led to an increase in prevalence of prodromal AD of more than $10 \%$. However, progression rates remained similar (data not shown).

The NIA-AA criteria differentiated between amyloid markers and neuronal injury markers and related the number of abnormal biomarkers to the likelihood of that MCI is due to AD. Our results showed that this is beneficial for diagnosis and prognosis since subjects with both abnormal amyloid and neuronal injury markers were almost eight times more likely to develop AD-type dementia compared to those with both normal amyloid and neuronal injury markers. Subjects with one of the two biomarkers abnormal were two to three times more likely to develop AD-type dementia than subjects with both normal biomarkers. Some subjects only had an injury marker available (intermediate or inconclusive group). In that case, the outcome can be very heterogeneous because some have $\mathrm{AD}$, i.e. amyloid pathology, and others have not. In our study, the intermediate group 
showed a progression rate to AD-type dementia that was similar to that of the conflicting biomarker groups together.

The similar progression rates to AD-type dementia of the conflicting amyloid and neuronal injury groups were particularly surprising, as subjects with SNAP (abnormal injury marker and normal amyloid marker) are expected to have lower progression rates than those with an abnormal amyloid marker and normal injury marker. ${ }^{18}$ Interestingly, subjects with SNAP who progressed to AD-type dementia had CSF A $\beta 1-42$ levels closer to the cut-off value. It could be that these subjects have more comorbidities so that less amyloid pathology is needed to progress to AD-type dementia. ${ }^{19}$ Alternatively, SNAP could be an atypical form of $\mathrm{AD}$ with less pronounced amyloid pathology. It is also possible that the CSF A 1-42 cut-offs used at each center were not optimal to identify all subjects with prodromal AD.

The NIA-AA criteria do not differentiate between the several neuronal injury markers. We showed that subjects with abnormal MRI in combination with an abnormal amyloid marker had higher progression rates to AD-type dementia than subjects with abnormal CSF tau in combination with abnormal amyloid marker. This is in line with the hypothetical model of AD biomarkers, ${ }^{20,21}$ which states that subjects with abnormal MRI are further in the disease process than those with abnormal CSF tau. Moreover, in a subgroup of subjects who had two injury markers available, we found that abnormality of both CSF tau and MRI in combination with an abnormal amyloid marker led to higher progression rates compared to only one abnormal injury marker in combination with an abnormal amyloid marker, suggesting that the former group is even further in the disease process. When we compared the predictive ability of conflicting injury markers, we found that abnormal CSF tau and normal MRI in combination with an abnormal amyloid marker had a similar predictive ability for AD-type dementia as normal tau and abnormal MRI in combination with abnormal amyloid marker. It may be that subjects with abnormal MRI had CSF tau levels just below the cut-off. Alternatively, tau and MRI abnormality could be parallel rather than serial pathways in the disease process.

In order to apply the IWG or NIA-AA criteria, the use of cut-offs for biomarkers and cognition is required. We chose to define cognitive impairment as 1.5 SD below the mean or equivalent score and used routine biomarker cut-offs of each center to define abnormal biomarkers. However, the use of different cut-off could have led to different results, which is a well-known drawback of using dichotomous data and also highlights the need for standardization of cut-offs.

This study had several limitations. No autopsy data were available, which might have led to diagnostic AD misclassifications. Furthermore, different centers used different cognitive tests and biomarker assessment protocols. This might have introduced heterogeneity between centers but findings may better reflect current clinical practice. Moreover, we corrected for center in statistical analyses and used Z-scores for cognitive tests defined at each center. The major strength of our study is the large sample size of wellcharacterized subjects. 
Our results have several implications. First, they show that prodromal AD can be identified based on biomarkers and the IWG and NIA-AA criteria. As these two sets of criteria showed good predictive ability for future cognitive decline they may be useful for selection of subjects for therapeutic trials. Second, knowledge about both amyloid pathology and neuronal injury is preferred to increase the certainty whether someone will develop ADtype dementia or not. Although a longer follow-up is needed to see whether all subjects with only amyloid pathology will become demented. Moreover, the use of multiple neuronal injury markers in combination with an amyloid marker can help to identify the subjects that will soon progress to AD-type dementia. Third, our findings suggest that a more lenient CSF A 1-42 cut-off may be needed to identify subjects with SNAP who have prodromal AD.

Together, our findings support the use of biomarker subgroups of the IWG and NIA-AA criteria for MCI, given its good predictive ability for future cognitive decline. Our results suggest that knowledge about both amyloid pathology and neuronal injury is preferred to give an optimal prognosis. More research on conflicting biomarkers and on longitudinal biomarker data is needed for final validation of the use of biomarkers in clinical practice. 


\section{REFERENCES}

1. Petersen RC. Mild cognitive impairment as a diagnostic entity. J Intern Med 2004;256:183-194.

2. Schneider JA, Arvanitakis Z, Leurgans SE, Bennett DA. The neuropathology of probable Alzheimer disease and mild cognitive impairment. Annals of neurology 2009;66:200-208.

3. Visser PJ, Verhey F, Knol DL, et al. Prevalence and prognostic value of CSF markers of Alzheimer's disease pathology in patients with subjective cognitive impairment or mild cognitive impairment in the DESCRIPA study: a prospective cohort study. Lancet neurology 2009;8:619-627.

4. Dubois B, Feldman HH, Jacova C, et al. Research criteria for the diagnosis of Alzheimer's disease: revising the NINCDS-ADRDA criteria. Lancet neurology 2007;6:734-746.

5. Dubois B, Feldman HH, Jacova C, et al. Revising the definition of Alzheimer's disease: a new lexicon. Lancet neurology 2010;9:1118-1127.

6. Albert MS, DeKosky ST, Dickson D, et al. The diagnosis of mild cognitive impairment due to Alzheimer's disease: recommendations from the National Institute on Aging-Alzheimer's Association workgroups on diagnostic guidelines for Alzheimer's disease. Alzheimer's \& dementia : the journal of the Alzheimer's Association 2011;7:270-279.

7. Oksengard AR, Cavallin L, Axelsson R, et al. Lack of accuracy for the proposed 'Dubois criteria' in Alzheimer's disease: a validation study from the Swedish brain power initiative. Dementia and geriatric cognitive disorders 2010;30:374-380.

8. Prestia A, Caroli A, van der Flier WM, et al. Prediction of dementia in MCI patients based on core diagnostic markers for Alzheimer disease. Neurology 2013;80:1048-1056.

9. Petersen RC, Aisen P, Boeve BF, et al. Criteria for mild cognitive impairment due to alzheimer's disease in the community. Annals of neurology 2013.

10. Visser PJ, Verhey FR, Boada M, et al. Development of screening guidelines and clinical criteria for predementia Alzheimer's disease. The DESCRIPA Study. Neuroepidemiology 2008;30:254-265.

11. Lovestone S, Francis P, Kloszewska I, et al. AddNeuroMed--the European collaboration for the discovery of novel biomarkers for Alzheimer's disease. Annals of the New York Academy of Sciences 2009;1180:36-46.

12. Morbelli S, Piccardo A, Villavecchia G, et al. Mapping brain morphological and functional conversion patterns in amnestic MCI: a voxel-based MRI and FDG-PET study. European journal of nuclear medicine and molecular imaging 2010;37:36-45.

13. Mueller SG, Weiner MW, Thal LJ, et al. Ways toward an early diagnosis in Alzheimer's disease: the Alzheimer's Disease Neuroimaging Initiative (ADNI). Alzheimer's \& dementia : the journal of the Alzheimer's Association 2005;1:55-66.

14. American Psychiatric Association. Diagnostic and Statistical Manual of Mental Disorders: DSM-IV. Washington, DC: American Psychiatric Association; 1994.

15. McKhann G, Drachman D, Folstein M, Katzman R, Price D, Stadlan EM. Clinical diagnosis of Alzheimer's disease: report of the NINCDS-ADRDA Work Group under the auspices of Department of Health and Human Services Task Force on Alzheimer's Disease. Neurology 1984;34:939-944.

16. Jack CR, Jr., Knopman DS, Weigand SD, et al. An operational approach to National Institute on AgingAlzheimer's Association criteria for preclinical Alzheimer disease. Annals of neurology 2012;71:765-775.

17. Vos SJ, van Rossum IA, Verhey F, et al. Prediction of Alzheimer disease in subjects with amnestic and nonamnestic MCI. Neurology 2013;80:1124-1132.

18. Knopman DS, Jack CR, Wiste HJ, et al. Short-term clinical outcomes for stages of NIA-AA preclinical Alzheimer disease. Neurology 2012;78:1576-1582. 
19. Jack CR, Wiste HJ, Vemuri P, et al. Brain beta-amyloid measures and magnetic resonance imaging atrophy both predict time-to-progression from mild cognitive impairment to Alzheimer's disease. Brain 2010;133:3336-3348.

20. Jack CR, Jr., Knopman DS, Jagust WJ, et al. Hypothetical model of dynamic biomarkers of the Alzheimer's pathological cascade. Lancet neurology 2010;9:119-128.

21. Jack CR, Jr., Knopman DS, Jagust WJ, et al. Tracking pathophysiological processes in Alzheimer's disease: an updated hypothetical model of dynamic biomarkers. Lancet neurology 2013;12:207216. 


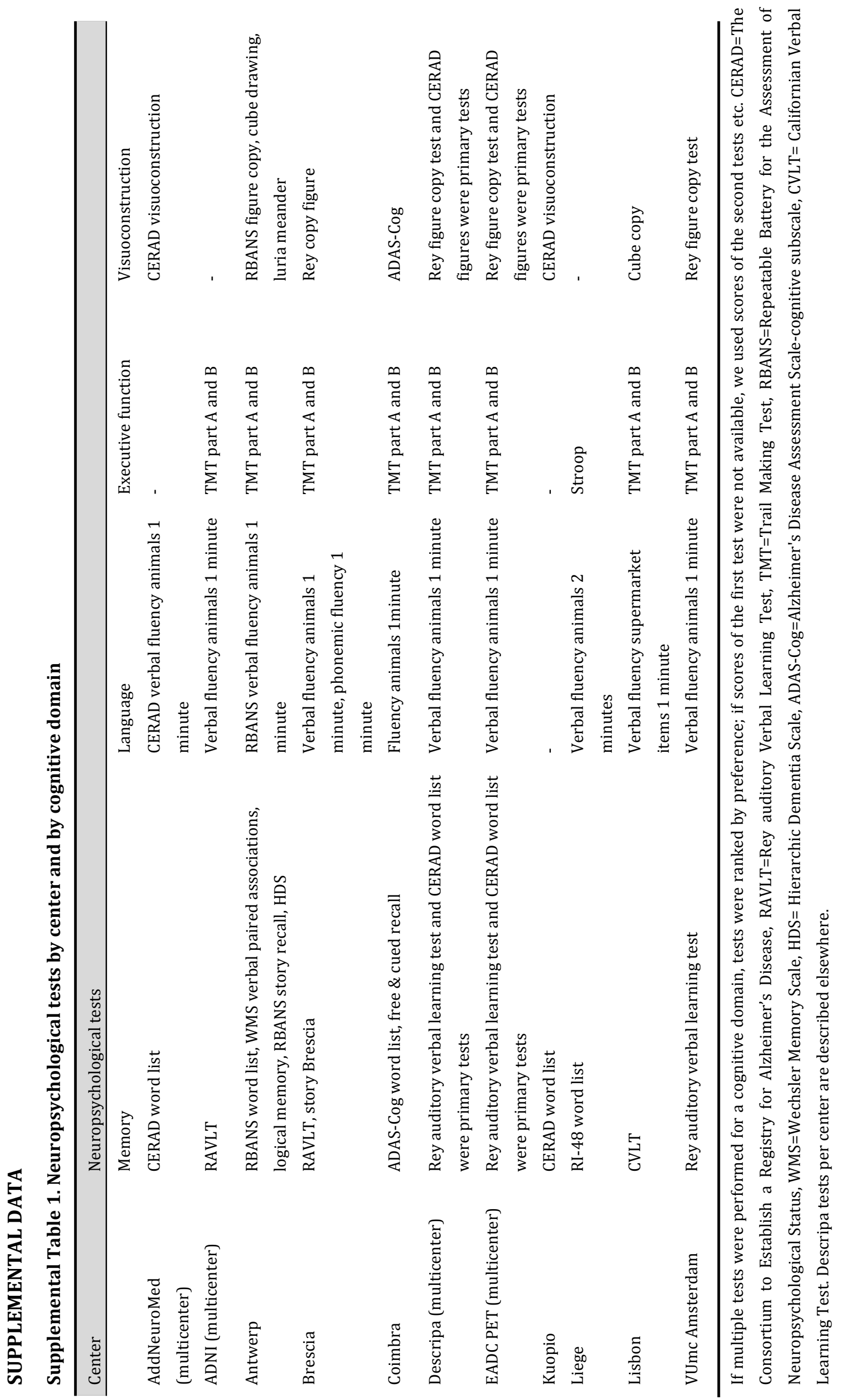




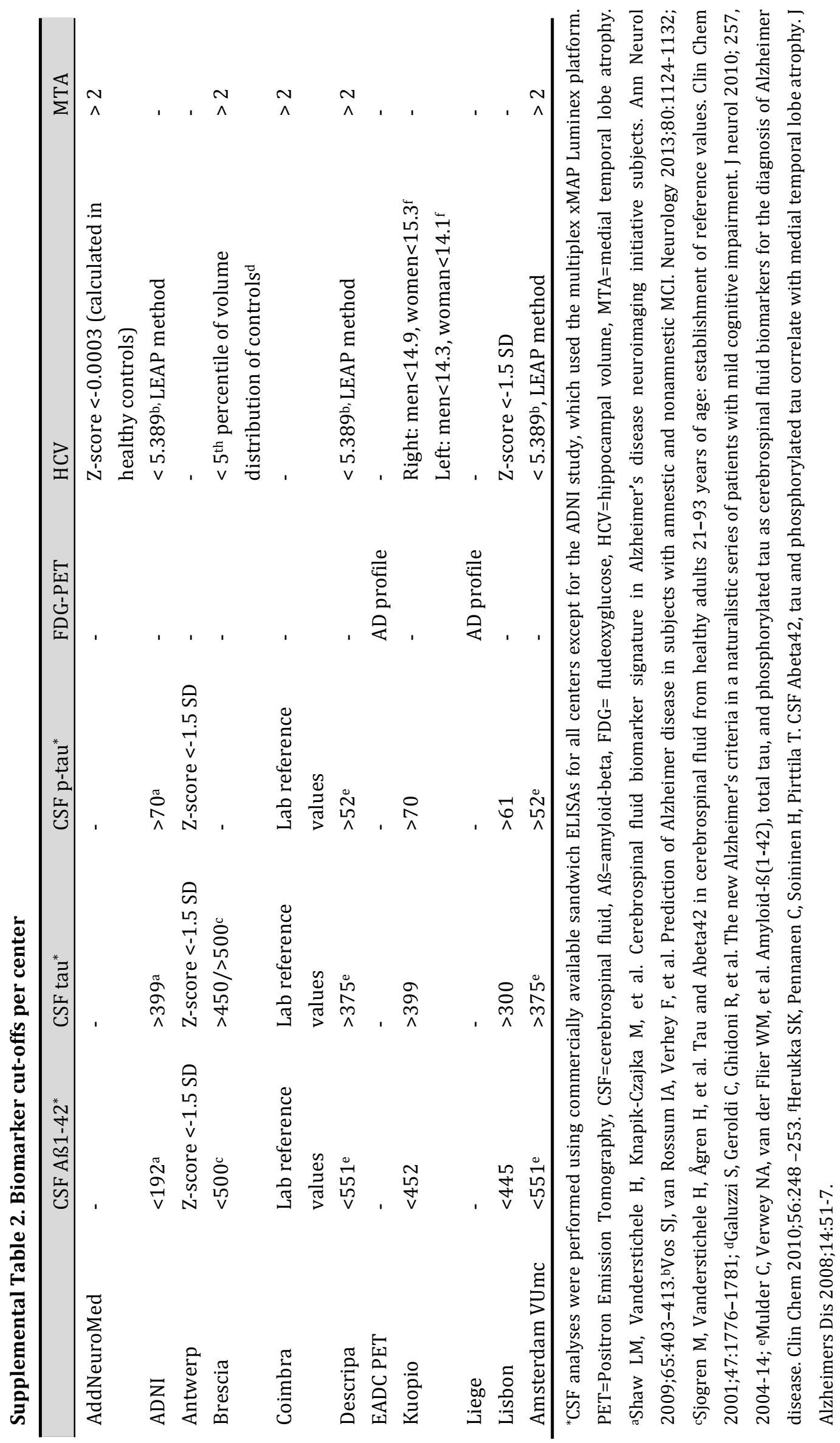


Supplemental Table 3 . Number of subjects by center and by biomarker

\begin{tabular}{|c|c|c|c|c|c|c|}
\hline \multirow[t]{2}{*}{ Center } & \multirow[t]{2}{*}{ Total N } & \multirow{2}{*}{$\begin{array}{l}\begin{array}{l}\text { Amyloid } \\
\text { marker }\end{array} \\
\text { CSF Aß1-42 }\end{array}$} & \multicolumn{4}{|c|}{ Injury marker } \\
\hline & & & CSF tau & $\begin{array}{l}\text { FDG- } \\
\text { PET }\end{array}$ & $\mathrm{HCV}$ & MTA \\
\hline AddNeuroMed & 99 & - & - & - & 98 & 85 \\
\hline ADNI & 300 & 155 & 155 & - & 300 & - \\
\hline Antwerp & 41 & 41 & 41 & - & - & - \\
\hline Brescia & 94 & 66 & 66 & - & 90 & 70 \\
\hline Coimbra & 37 & 17 & 17 & - & - & 36 \\
\hline Descripa & 270 & 104 & 104 & - & 214 & 235 \\
\hline EADC PET & 143 & - & - & 143 & - & - \\
\hline Kuopio & 50 & 19 & 19 & - & 48 & - \\
\hline Liege & 49 & - & - & 49 & - & - \\
\hline Lisbon & 36 & 2 & 2 & - & 36 & - \\
\hline Amsterdam VUmc & 126 & 90 & 90 & - & 98 & 112 \\
\hline
\end{tabular}

$\mathrm{CSF}=$ cerebrospinal fluid, $\mathrm{A} ß=$ amyloid-beta, $\mathrm{FDG}=$ fludeoxyglucose, $\mathrm{PET}=$ Positron Emission Tomography, $\mathrm{HCV}=$ hippocampal volume, MTA=medial temporal lobe atrophy, EADC=European Alzheimer's Disease Consortium. 


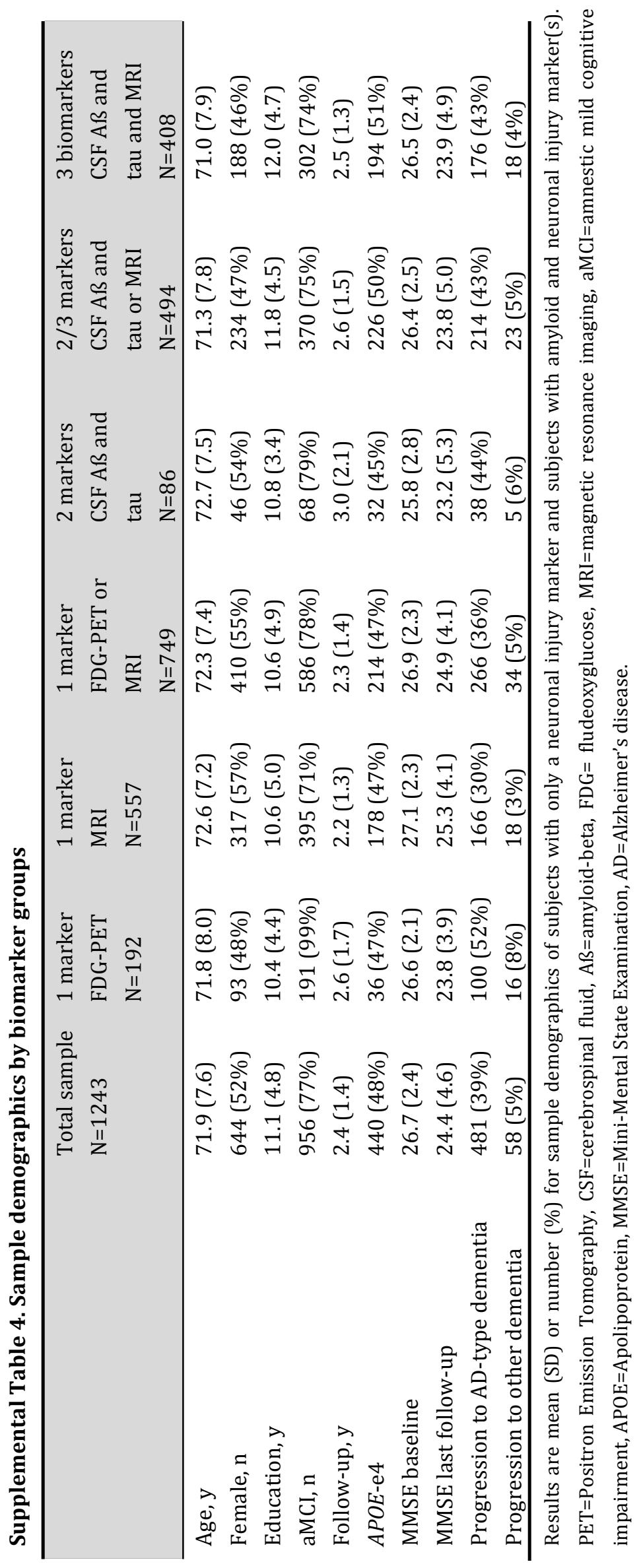




\title{
CHAPTER 10
}

\author{
GENERAL DISCUSSION \\ SUMMARY \\ NEDERLANDSE SAMENVATTING \\ AUTHOR AFFILIATIONS \\ PUBLICATIONS \\ AUTHOR INFORMATION \\ ACKNOWLEDGEMENTS
}




\section{GENERAL DISCUSSION}

The Alzheimer's disease field is experiencing an exciting evolution with biomarkers at the forefront. For a long time, AD has been considered a clinicopathological disease and the clinical diagnosis has not been taken into account the long preclinical and prodromal stages of AD. Thanks to advances in the AD biomarker field, our understanding of the early course of $\mathrm{AD}$ has substantially improved over the past years. $\mathrm{AD}$ is now regarded as a clinicobiological disease that can be identified in vivo prior to the onset of dementia.

The aim of this thesis was to investigate how we should use biomarkers for early diagnosis and prognosis of Alzheimer's disease. For this purpose, in part I of this thesis, we examined the role of AD biomarkers in subjects with MCI. In part II of this thesis, we studied the prevalence and prognosis of preclinical and prodromal $\mathrm{AD}$, according to the recently proposed IWG and NIA-AA criteria for AD. We will now discuss our findings and the implications for research, AD trials and clinical practice. We will conclude with recommendations for future research.

\section{Diagnostic and prognostic value of AD biomarkers in subjects with MCI}

$A D$ markers for prediction of AD-type dementia in subjects with $M C I$

Several AD markers are available but it is still unclear which is the optimal biomarker for predicting progression from MCI to AD-type dementia. Our studies indicated that the CSF Aß1-42/tau ratio was a better predictor than CSF Aß1-42, CSF tau, and HCV. In chapter 2, we compared the predictive ability of CSF Aß1-42, $t$-tau, $p$-tau, and a combined measure of Aß1-42 and tau for AD-type dementia in subjects with MCI by means of a meta-analysis. We found that all single markers had a similar predictive value with an odds ratio around 7 , whereas the predictive value of the combination of CSF Aß1-42 and tau was more than twice as high (odds ratio 18). This is in line with previous studies. ${ }^{1,2}$ We also compared the predictive ability of CSF Aß1-42/tau ratio with that of HCV on MRI for AD-type dementia after 2 years and found that CSF and MRI markers were both good predictors, independent of cognition and APOE genotype (chapter 3). However, the CSF Aß1-42/tau ratio had a higher sensitivity and an overall predictive value that was more than twice as high than that of HCV. Several previous studies also found that CSF markers were a better predictor for progression to AD-type dementia than MRI markers ${ }^{3-6}$ while other studies found that MRI markers were the best predictor. ${ }^{7-9}$ In chapter 4 , we showed that CSF Aß1-42, CSF tau, and HCV all had a similar predictive accuracy for AD-type dementia and the CSF Aß1-42/tau ratio was again the best predictor. The better performance of the CSF Aß1-42/tau ratio compared to other CSF markers and HCV is probably because information on an early AD process (amyloid pathology) will help to identify those with $\mathrm{AD}$ and additional information on a more downstream process (tau pathology) will help to give an appropriate prognosis. ${ }^{10}$, 11

In Chapter 4, we compared the predictive ability of CSF and MRI markers and APOEgenotype for AD-type dementia in subjects with aMCI and naMCI because AD pathology may 
present differently in both MCI subgroups and therefore AD biomarkers may have a different predictive accuracy for AD-type dementia. While progression rates to AD-type dementia are known to be higher in aMCI,12,13 we demonstrated that AD markers were equally predictive in both MCI subgroups. However, sensitivity of CSF and MRI markers was higher and specificity lower in subjects with aMCI. This was mainly because subjects with aMCI had more abnormal biomarker scores than subjects with naMCI. AD biomarkers may therefore not be as sensitive for early diagnosis of AD in subjects with naMCI compared to aMCI when the same cut-offs are applied in each subgroup. This is particularly interesting in light of the proposed NIA-AA criteria for MCI due to $\mathrm{AD},{ }^{14}$ as they consider both aMCI and naMCI as possible prodromal stages of AD-type dementia.

\section{Combination of $A D$ markers for prediction of AD-type dementia in subjects with $M C I$}

Instead of investigating single markers for $A D$, it may be more beneficial to combine several markers for optimal diagnosis and prognosis of AD. While the CSF Aß1-42/tau ratio was the best predictor in our MCI studies, the combination with HCV increased predictive accuracy for AD-type dementia (chapter 3), as also shown in other studies., 4, 7-9 This is likely because both markers reflect different underlying $\mathrm{AD}$ pathology and become abnormal at different stages of the disease. Our test sequence analyses suggested that it is most efficient to perform first a CSF assessment for prediction of AD-type dementia after 2 years. Volumetric assessment of hippocampal volume may be considered in subjects who have a normal CSF Aß1-42/tau, mainly because we found a small group of subjects with a normal CSF ratio and abnormal HCV that progressed to AD-type dementia ( $\mathrm{n}=5)$.

In chapter 5, we reported that abnormal CSF t-tau, CSF p-tau, and HCV are good markers of faster future cognitive decline and progression to AD-type dementia in subjects with MCI and CSF amyloid pathology, i.e. prodromal AD. This is in line with a previous study ${ }^{15}$ and highlights again the importance of combining neuronal injury markers with amyloid markers for better prognosis. We did not find large differences in predictive ability between injury markers but results in chapter 5 and 9 showed that subjects with two abnormal injury markers and amyloid pathology were more likely to progress to AD-type dementia compared to those with only one abnormal injury marker and amyloid pathology. Overall, because of the early position of amyloid markers in the AD pathological cascade, they may have a better diagnostic value, whereas neuronal injury markers are later markers and may therefore have a better prognostic value. ${ }^{16-19}$

\section{New diagnostic criteria for preclinical and prodromal Alzheimer's disease}

The introduction of the concepts preclinical and prodromal AD in the revised criteria for $\mathrm{AD}^{14,20-22}$ is interesting both from a diagnostic and therapeutic perspective, as we can identify AD now before the dementia stage, i.e. at the predementia stages. The classification of subjects based on both cognitive and biomarker status helps to increase the certainty of whether a subject has underlying AD pathology or not and, if so, determines what disease stage the subject is at and aids to make a prognosis. 


\section{NIA-AA criteria for preclinical $A D$}

In chapter 8, we showed that the use of CSF markers and episodic memory according to the NIA-AA criteria for preclinical AD enables identification of subjects with preclinical AD. We found that $31 \%$ of the cognitively healthy participants met criteria for preclinical AD, with $15 \%$ of the subjects in stage $1,12 \%$ in stage 2 , and $4 \%$ in stage 3 . These findings together with our autopsy data provide support for the accumulation of AD pathology in cognitively healthy elderly already. ${ }^{23-25}$ Furthermore, the preclinical AD stages strongly predicted cognitive decline, progression to CDR 0.5 that clinicians believed was due to AD, and also mortality. Subjects in a more advanced preclinical AD stage were more likely to progress to CDR 0.5 symptomatic AD, as also shown in a previous study. ${ }^{26}$

Even though these criteria seem promising for identifying preclinical $A D$, they have some limitations. Not all subjects with $\mathrm{AD}$ fit within the proposed classification groups. ${ }^{27} 5 \%$ of the subjects remained unclassified based on the NIA-AA criteria (chapter 8). The majority of these subjects had subtle cognitive changes in the presence of normal biomarkers whereas a few had also amyloid pathology. As this group was likely to progress to AD-type dementia (29\%), the underlying pathology of these subjects needs further investigation. Furthermore, the criteria did not specify subtle cognitive decline in detail to define preclinical AD stage 3, which leads to possible variability. Using any cognitive domain versus episodic memory to define stage 3 led to different results in chapter 8 . The number of subjects in stage 3 and the unclassified group was larger if any cognitive domain was used but progression rates were lower than if episodic memory was used. Another problem is the need to use cut-offs to define biomarkers as normal vs. abnormal, as the use of different cutoffs may lead to different findings. Results in chapter 8 showed that a more lenient cut-off will lead to more subjects identified with preclinical $\mathrm{AD}$, however progression rates remained essentially the same.

\section{IWG and NIA-AA criteria for prodromal AD}

In chapter 9, we showed that prodromal AD was common and that these subjects showed much higher progression rates to AD-type dementia compared to those without prodromal AD. According to the IWG criteria, $54 \%$ of the subjects had prodromal AD and these subjects showed more cognitive decline than those with normal biomarkers. According to the NIAAA criteria, $51 \%$ of the subjects were in the high-AD-likelihood group (both amyloid pathology and neuronal injury), 15\% in the low-AD-likelihood group (normal markers), 6\% in the IAP conflicting group (amyloid pathology without neuronal injury), and 29\% were in the SNAP conflicting group (neuronal injury without amyloid injury). As expected, those with the highest likelihood showed the highest progression rates for AD-type dementia $(62 \%)$ and those with the lowest likelihood the lowest progression rates (14\%). Subjects in the IAP and SNAP groups showed intermediate and similar progression rates (24-30\%). This latter finding was unexpected given their underlying pathology.

In their present form, the NIA-AA criteria may be favored over the IWG criteria for a few reasons. The NIA-AA criteria suppose that an increased number of abnormal 
biomarkers is related to an increased likelihood that $\mathrm{MCI}$ is due to $\mathrm{AD},{ }^{14}$ which was indeed the case in our study. In contrast, the IWG criteria require only one abnormal biomarker (any) for prodromal AD and only an abnormal amyloid marker for asymptomatic subjects at risk for $\mathrm{AD},{ }^{20,21}$ which makes identification of subjects who are most likely to progress soon to AD-type dementia more difficult. For instance, we cannot tell whether subjects with amyloid pathology without information on neuronal injury are at an early or later process of the disease. Also, subjects with neuronal injury without information on amyloid pathology may have $\mathrm{AD}$ but may also have other diseases. Another important difference is the definition of cognition. The IWG criteria require episodic memory impairment while the NIA-AA criteria do not specify cognitive impairment. Our results in chapter 4 show that also subjects with naMCI can progress to AD-type dementia, although the progression rates are lower. Again, the use of biomarker cut-offs is also a limitation for these both sets of criteria.

\section{Standardization of AD biomarkers}

AD biomarkers are increasingly being used in clinical practice but the absence of standardized analyses for biomarkers constitutes a major deficiency and may influence the diagnostic AD classification of subjects. Findings in chapter 6 show that there is still considerable intra-and interlaboratory variability of CSF analyses, consistent with previous reports. ${ }^{28-32} \mathrm{~A}$ substantial part of the observed variability may be explained by lot-to-lot variation of analytical kits but future studies on lot-to-lot comparison will need to confirm this suggestion. Moreover, we found that the variability frequently influences AD diagnosis when predefined cut-offs were used to classify CSF biomarkers as normal or abnormal. Importantly, also a diagnosis of a CSF AD profile based on A $\beta 1-42$ and t-tau or p-tau changed in $12-16 \%$ of the cases. While most of the subjects in our study with a change in AD classification after reanalysis had CSF biomarker values around the cut-off points, the variability may have a large impact on CSF-based AD diagnosis or treatment decisions in clinical settings, implying that we should be careful when interpreting CSF findings and always interpret them within a clinical context. Another point of concern is the use of cutoffs to define the continuous biomarker scores as normal or abnormal. An agreement on universal cut-offs is needed, ${ }^{33}$ because the use of different cut-offs can lead to different diagnoses in patients (chapter 6,8). Other studies have shown that also imaging markers still suffer from poor standardization and reproducibility. ${ }^{34}$ Ongoing ${ }^{30,} 35$ and future worldwide standardization projects will help to move towards a standardized and harmonized implementation of AD biomarkers in clinical routine.

\section{Methodological considerations}

Strengths

The use of large sample sizes increased the power of our studies and allowed us to perform multivariate analyses. The longitudinal study designs with up to 2 (chapter 3), 5 (chapter 4 , 5), 9 (chapter 9), and 15 years (chapter 8) of follow-up gave us the opportunity to investigate the short-term as well as long-term prognosis of subjects. By means of a meta- 
analysis we could get insight into the predictive ability of CSF biomarkers by pooling data from previous studies and taking into account the sample size of all studies (chapter 2).

\section{Limitations}

Our studies had several limitations. Because subjects agreed to take part in longitudinal studies, including neuroimaging procedures and lumbar punctures, they are unlikely to be representative of the general population. Subjects were also relatively young elderly and findings may therefore not apply to the oldest old. The DESCRIPA study (chapter 3-5) and EADC study (chapter 9) were multi-center memory clinic-based studies, which allowed generalization to other memory clinics but not to the general population. In contrast, data from the community-dwelling-volunteer-based study of Washington University in St. Louis (chapter 8) allowed generalization to a broader population although it may be restricted to Caucasians and highly educated people. Furthermore, the DESCRIPA and EADC study were based on clinical routine rather than standardized protocols. This might have introduced heterogeneity between centers but findings may better reflect current clinical practice. Moreover, we corrected for center in statistical analyses and used Z-scores or equivalent scores for cognitive tests defined at each center. In the DESCRIPA study, biomarker analyses were also performed at one center.

Other limitations were the loss to follow-up and missing data, because of which valuable information could have been missing. Although follow-up was relatively long, a longer follow-up would have helped to investigate whether all subjects with abnormal biomarkers eventually will develop AD-type dementia. Moreover, we used clinical diagnoses as outcome measure instead of autopsy diagnoses (except for a small subgroup in chapter 8), which may have led to misclassification of subjects and which could have hampered the validation of biomarkers. Furthermore, multiple testing could be a problem in some studies but we mostly provided p-values to correct for multiple testing. In addition, estimation of temporal dynamics in biomarkers changes was based on cross-sectional biomarker studies but longitudinal biomarker data are needed to examine this thoroughly.

\section{IMPLICATIONS OF AD BIOMARKERS}

\section{Amyloid cascade hypothesis}

Findings in this thesis may have implications for the amyloid cascade hypothesis, ${ }^{36,37}$ which has dominated research for the past two decades. This hypothesis states that AD pathophysiology starts with amyloid deposition already 10 to 15 years before the first clinical symptoms appear, which initiates multiple downstream events leading to neuronal injury and eventually to clinical symptoms. In line with this, Jack and colleagues recently proposed a hypothetical AD biomarker model, which suggests that abnormal CSF Aß1-42 can be detected first, followed by amyloid PET, CSF tau, and MRI and FDG PET. ${ }^{38,} 39$ Our findings are partially in favour of the amyloid cascade and the hypothetical biomarker model. We indeed found that subjects with both amyloid deposition and neuronal injury 
showed more cognitive decline than those with only amyloid pathology, likely because they are further in the disease process. Furthermore, the higher sensitivity of the CSF Aß142/tau ratio for progression to AD-type dementia than that of HCV may indicate that CSF markers become abnormal at an earlier stage than MRI biomarkers. However, we also recognized some inconsistencies with regard to the model. We did not find a difference in overall predictive accuracy between the single CSF markers and HCV. Several subjects had hippocampal atrophy but normal CSF tau, which may suggests that pathways are not the same for all subjects. Also, subjects with normal CSF Aß1-42 and abnormal CSF tau or HCV had an increased risk for AD-type dementia, but as discussed below this CSF Aß1-42 classification may partly be a cut-off issue. Therefore, while the cascade is a useful model, careful interpretation of amyloid and neuronal injury markers is likely needed for diagnosing and staging AD.

\section{Isolated neuronal injury}

Some of the subjects in our study did not fit within the amyloid cascade because they had neuronal injury without amyloid pathology. At present, they are therefore suggested to have suspected non-Alzheimer pathophysiology (SNAP). ${ }^{27}$ Both in cognitively healthy controls and in subjects with MCI, the SNAP group represented a large group of subjects (23-29\%). Interestingly, cognitively healthy controls with SNAP did not progress to AD-type dementia or showed substantial cognitive decline at follow-up (chapter 8), consistent with a previous study. ${ }^{26}$ They were less likely to be APOE- $\varepsilon 4$ carriers than subjects with preclinical AD but they do not appear to differ regarding cerebrovascular imaging lesions or vascular risk factors. ${ }^{26}$ Because of low progression rates to AD-type dementia, they may have other neurodegenerative diseases. Our autopsy data showed that they had no to low AD pathology change at autopsy and other pathologies included argyrophilic grain disease, neurofibrillary tangles in medial temporal lobe, or small vessel disease with infarcts/microinfarcts. These pathologies were however not sufficient to account for cognitive impairments. It could also be that tau pathology reflects normal cognitive aging in these subjects.

We and others ${ }^{40}$ found that subjects with MCI and SNAP showed progression rates to AD-type dementia similar to those with isolated amyloid pathology but they tended to be less likely $A P O E-\varepsilon 4$ carriers than those with prodromal AD with amyloid pathology (chapter 9). Intriguingly, subjects with MCI and SNAP who progressed to AD-type dementia had intermediate CSF Aß1-42 levels (just above the predefined cut-off), and they may therefore reflect a subgroup of subjects for whom less amyloid pathology is needed to develop ADtype dementia (chapter 9). This could also explain our findings in chapter 3 , where MRI analyses could only add to the predictive accuracy for AD-type dementia in a small group of subjects with MCI with a normal CSF Aß1-42/tau ratio. Remarkably, most of these subjects that progressed to AD-type dementia also appeared to have rather intermediate CSF Aß1-42 levels in the presence of abnormal tau. The intermediate CSF Aß1-42 levels in subjects with MCI and SNAP and relatively high progression rates to AD-type dementia (30\%) after a mean follow-up of 3 years suggest that they may have atypical AD. This could be explained 
by the variety in CSF Aß1-42 levels in cognitively healthy elderly or by the variability in type of aggregated $A{ }^{41}{ }^{41}$ It may also result from lower cognitive reserve ${ }^{42}$ or from pathological heterogeneity because of multicomorbitidy. ${ }^{17,43}$ Overall, the high prevalence of SNAP in healthy controls as well as in subjects with MCI and the high progression rate to AD-type dementia in this latter group highlight the importance to further explore the comorbid diseases, underlying pathology, clinical characteristics and outcome of these subjects.

\section{Clinical trials}

The identification of preclinical and prodromal AD is particularly interesting for AD trials, as development of therapeutic strategies is now aimed at truly modifying the underlying disease pathogenic processes rather than simply ameliorating dementia symptoms. Inclusion of subjects with preclinical and prodromal AD will ensure appropriate therapeutic targeting and will lead to higher progression rates to AD-type dementia and therefore smaller sample sizes (chapter 2). Age and APOE genotype may be useful for prescreening of individuals for biomarker assessment, ${ }^{44}$ given the higher prevalence of $A D$ pathology in $A P O E-\varepsilon 4$ carriers $^{45}$ or older subjects, ${ }^{46}$ as also shown in chapter 8 . Furthermore, trials may stratify individuals based on their predementia $\mathrm{AD}$ stage $\mathrm{e}^{47}$ and number of abnormal biomarkers since their prevalence and prognosis differ (chapter 8, 9). We showed that subjects at a more advanced stage will progress faster to AD-type dementia and therefore a smaller sample size is needed for trials. However, a larger number will be required for screening given the lower prevalence with advancing stage (chapter 3, 8, 9). Also, neuronal injury markers could be used as outcome in AD trials $48,49,50$ since they are closely related to cognition and may be more reliable measures of AD pathophysiological changes than cognitive outcomes. Amyloid markers could be used as outcome to provide efficacy of the intervention. ${ }^{51,52}$ Moreover, our study in preclinical AD suggests that mortality should be considered as an outcome in trials, as subjects with preclinical AD had an increased risk to die at follow-up, with a higher risk at more advanced stages (chapter 8).

\section{Clinical practice}

Biomarker assessment is currently complementary and recommended in case of substantial clinical uncertainty after other diagnostic tests. ${ }^{53}$, 54 However, as biomarker research progresses, also biomarker assessment in early $\mathrm{AD}$ stages becomes more common in clinical practice, albeit still controversial. An important advantage of diagnosis at the prodromal AD stage is that it helps the patient and family to understand and accept the underlying cause of their cognitive problems, which reduces uncertainty and in this way may increase quality of life. Patients will also be more likely to get earlier appropriate care and they can anticipate the future. However, there are also several disadvantages. The main drawback is that there is currently no effective treatment. ${ }^{55}$ An early diagnosis my also provoke stress and catastrophic reactions. Furthermore, no clear prognosis can be given to patients with prodromal $\mathrm{AD}$, as the time to dementia is uncertain and not all subjects may become demented. ${ }^{56}$ Both compensatory mechanisms and cognitive reserve may influence the 
progression to dementia. ${ }^{42,57}$ Moreover, the prevalence of other brain pathologies could have impact on the relationship between AD pathology and clinical symptoms, 58 59, 60 which is especially common at an older age. ${ }^{61}$

A diagnosis of $\mathrm{AD}$ at the preclinical stage is currently restricted to research settings. As these subjects have no cognitive problems and there is no effective treatment available, an early diagnosis raises lots of ethical questions because it may do more harm than good to these subjects. Once preventive treatments become available, biomarkers will start playing a more prominent role in preclinical AD. A plasma biomarker for AD would be ideal then but so far attempts to develop an accurate plasma AD marker have been unsuccessful (chapter 2). ${ }^{62}$

Our data suggest that we should differentiate between upstream and more downstream markers of $\mathrm{AD}$ to give the best diagnosis and prognosis (chapter 3-5,8,9). For amyloid diagnosis, amyloid PET imaging or CSF Aß1-42 can be used. When we compare both markers, amyloid imaging may be less invasive and there may be less variation across analyses, while CSF analyses are less expensive and subjects are not exposed to radiation. Importantly, some studies have shown that amyloid pathology can be detected in CSF before it is seen on amyloid PET scans ${ }^{63,64}$ but more research is needed to confirm this. It should also be noted that PET amyloid markers primarily measure insoluble fibrillar forms of $A B$, while reduced $A ß$ in CSF may also reflect aggregation of other $A ß$ species such as oligomers. Besides, clinicians should bear in mind that amyloid pathology is not specific for AD, as it may also be seen in subjects with Lewy body dementia or vascular dementia. ${ }^{65,66}$ Results should therefore always be carefully interpreted in clinical context. Furthermore, an injury marker should be used to give a prognosis. The advantage of using CSF markers is that they give info on both amyloid pathology $(\mathrm{A} \beta$ ) and neuronal injury (tau), and we showed that CSF tau is a good prognostic marker (chapter 5). The advantage of MRI is that it is often already routinely done to exclude other diseases and additional volumetric assessment of the hippocampus may be useful to predict outcome as shown in chapter 3 and 5. Our test sequence analyses suggest that CSF assessment should be performed first for short-term prediction of AD-type dementia and that HCV assessment may be considered in subjects who have a normal CSF Aß1-42 /tau ratio.

At present, the application of the new criteria for prodromal AD in clinical practice comes with several problems. It is for instance unclear what we should tell patients with conflicting biomarker results. We do not know yet whether all subjects with a SNAP biomarker profile (neuronal injury without amyloid pathology) have AD and will progress to AD-type dementia or not. This is also true for patients with isolated amyloid pathology, since maybe not all of them will progress to AD-type dementia over time. Moreover, we found that several subjects have values around the biomarker cut-off, which may influence their biomarker classification and diagnosis. The CSF Aß1-42/tau ratio may be a good alternative because it may catch more people with AD that just fall below the Aß1-42 or tau cut-offs when these markers are used separately. Before implementation of biomarkers in routine clinical practice, standardized and harmonized procedures for biomarker 
assessment need to be developed (chapter 6) and the encountered difficulties with the revised AD criteria must be resolved (chapter $8 \& 9$ ).

The shift from diagnosing $\mathrm{AD}$ at the dementia stage towards diagnosing $\mathrm{AD}$ at the predementia stage might raise the question what $\mathrm{AD}$ actually is, i.e. the underlying pathophysiological disease process or the clinical syndrome of progressive cognitive decline. Until recently, the clinical diagnosis of probable AD-type dementia was the best alternative for definite AD diagnosis at autopsy. Now, biomarkers allow us to provide an earlier AD diagnosis independent of clinical expression of disease severity. However, several individuals with amyloid pathology will never develop any AD symptoms or AD-type dementia. ${ }^{56}$ Given the lack of effective treatment and individual risk prediction for AD-type dementia, one may wonder whether we should label someone with an AD diagnosis before they are demented, i.e. in need of care. For clinicians it is important to keep in mind that patients do not suffer from the pathology but from the cognitive impairments and that a diagnosis at the preclinical stage is therefore currently unwarranted in clinical settings. In contrast, a diagnosis of $\mathrm{AD}$ at the prodromal stage can help to explain the cause of their cognitive problems, even though several uncertainties about the future may persist. Nonetheless, biomarker use can lead to an incorrect classification of patients as being at high risk for AD-type dementia, i.e. false positives, which may have an adverse effect on the patient's well being. At present, the main advantage is that we can rule out AD in several patients with MCI that have normal biomarkers, which will provide relief in these patients. Overall, for each patient with MCI with uncertain etiology, the potentials and limitations of biomarker use should always be carefully discussed before application. ${ }^{67}$

\section{FUTURE DIRECTIONS}

The last decade, large improvements have been made in the AD field thanks to biomarker developments but several questions remain unanswered. It is not known yet which set of biomarkers can best diagnose AD and predict AD-type dementia over time and what would be the optimal test sequence. More studies on head-to-head comparison of markers in longitudinal clinical studies with an extensive follow-up and preferably also post-mortem examination will be needed. Also more longitudinal biomarker studies are required to get better insight into the temporal dynamics of AD biomarkers because currently most studies are based on cross-sectional biomarker analyses. Furthermore, the time to AD-type dementia differs between subjects and not all subjects with AD pathology develop AD-type dementia. This needs further exploration and may for instance relate to mixed pathologies and cognitive reserve. Also the factors that predispose someone to develop AD pathology should be studied more in detail, as vascular factors and lifestyle factors likely play a role in midlife already. In addition, isolated tau pathology needs more investigation, especially in light of our SNAP findings. All of this will help to better understand preclinical and prodromal AD, which may lead to better treatment opportunities in the future. 


\section{REFERENCES}

1. Hansson O, Zetterberg H, Buchhave P, Londos E, Blennow K, Minthon L. Association between CSF biomarkers and incipient Alzheimer's disease in patients with mild cognitive impairment: a followup study. Lancet neurology 2006;5:228-234.

2. Mattsson N, Zetterberg H, Hansson O, et al. CSF biomarkers and incipient Alzheimer disease in patients with mild cognitive impairment. JAMA : the journal of the American Medical Association 2009;302:385-393.

3. Bouwman FH, Schoonenboom SN, van der Flier WM, et al. CSF biomarkers and medial temporal lobe atrophy predict dementia in mild cognitive impairment. Neurobiology of aging 2007;28:10701074.

4. Eckerstrom C, Andreasson U, Olsson E, et al. Combination of hippocampal volume and cerebrospinal fluid biomarkers improves predictive value in mild cognitive impairment. Dementia and geriatric cognitive disorders 2010;29:294-300.

5. Galluzzi S, Geroldi C, Ghidoni R, et al. The new Alzheimer's criteria in a naturalistic series of patients with mild cognitive impairment. Journal of neurology 2010;257:2004-2014.

6. Landau SM, Harvey D, Madison CM, et al. Comparing predictors of conversion and decline in mild cognitive impairment. Neurology 2010;75:230-238.

7. Ewers M, Walsh C, Trojanowski JQ, et al. Prediction of conversion from mild cognitive impairment to Alzheimer's disease dementia based upon biomarkers and neuropsychological test performance. Neurobiology of aging 2012;33:1203-1214.

8. Vemuri P, Wiste HJ, Weigand SD, et al. MRI and CSF biomarkers in normal, MCI, and AD subjects: predicting future clinical change. Neurology 2009;73:294-301.

9. Heister D, Brewer JB, Magda S, Blennow K, McEvoy LK. Predicting MCI outcome with clinically available MRI and CSF biomarkers. Neurology 2011;77:1619-1628.

10. Fagan AM, Holtzman DM. Cerebrospinal fluid biomarkers of Alzheimer's disease. Biomarkers in medicine 2010;4:51-63.

11. Blennow K, Hampel H. CSF markers for incipient Alzheimer's disease. Lancet neurology 2003;2:605-613.

12. Petersen RC, Smith GE, Waring SC, Ivnik RJ, Tangalos EG, Kokmen E. Mild cognitive impairment: clinical characterization and outcome. Archives of neurology 1999;56:303-308.

13. Jungwirth S, Zehetmayer S, Hinterberger M, Tragl KH, Fischer P. The validity of amnestic MCI and non-amnestic MCI at age 75 in the prediction of Alzheimer's dementia and vascular dementia. International psychogeriatrics / IPA 2012;24:959-966.

14. Albert MS, DeKosky ST, Dickson D, et al. The diagnosis of mild cognitive impairment due to Alzheimer's disease: recommendations from the National Institute on Aging-Alzheimer's Association workgroups on diagnostic guidelines for Alzheimer's disease. Alzheimer's \& dementia : the journal of the Alzheimer's Association 2011;7:270-279.

15. Schott JM, Bartlett JW, Fox NC, Barnes J. Increased brain atrophy rates in cognitively normal older adults with low cerebrospinal fluid Abeta1-42. Annals of neurology 2010;68:825-834.

16. Ingelsson M, Fukumoto H, Newell KL, et al. Early Abeta accumulation and progressive synaptic loss, gliosis, and tangle formation in AD brain. Neurology 2004;62:925-931.

17. Jack CR, Wiste HJ, Vemuri P, et al. Brain beta-amyloid measures and magnetic resonance imaging atrophy both predict time-to-progression from mild cognitive impairment to Alzheimer's disease. Brain 2010;133:3336-3348. 
18. van Rossum IA, Visser PJ, Knol DL, et al. Injury markers but not amyloid markers are associated with rapid progression from mild cognitive impairment to dementia in Alzheimer's disease. Journal of Alzheimer's disease : JAD 2012;29:319-327.

19. Buchhave P, Minthon L, Zetterberg H, Wallin AK, Blennow K, Hansson O. Cerebrospinal fluid levels of beta-amyloid 1-42, but not of tau, are fully changed already 5 to 10 years before the onset of Alzheimer dementia. Archives of general psychiatry 2012;69:98-106.

20. Dubois B, Feldman HH, Jacova C, et al. Research criteria for the diagnosis of Alzheimer's disease: revising the NINCDS-ADRDA criteria. Lancet neurology 2007;6:734-746.

21. Dubois B, Feldman HH, Jacova C, et al. Revising the definition of Alzheimer's disease: a new lexicon. Lancet neurology 2010;9:1118-1127.

22. Sperling RA, Aisen PS, Beckett LA, et al. Toward defining the preclinical stages of Alzheimer's disease: recommendations from the National Institute on Aging-Alzheimer's Association workgroups on diagnostic guidelines for Alzheimer's disease. Alzheimer's \& dementia : the journal of the Alzheimer's Association 2011;7:280-292.

23. Price JL, Morris JC. Tangles and plaques in nondemented aging and "preclinical" Alzheimer's disease. Annals of neurology 1999;45:358-368.

24. Hulette CM, Welsh-Bohmer KA, Murray MG, Saunders AM, Mash DC, McIntyre LM. Neuropathological and neuropsychological changes in "normal" aging: evidence for preclinical Alzheimer disease in cognitively normal individuals. Journal of neuropathology and experimental neurology 1998;57:1168-1174.

25. Braak H, Braak E. Frequency of stages of Alzheimer-related lesions in different age categories. Neurobiology of aging 1997;18:351-357.

26. Knopman DS, Jack CR, Jr., Wiste HJ, et al. Short-term clinical outcomes for stages of NIA-AA preclinical Alzheimer disease. Neurology 2012;78:1576-1582.

27. Jack CR, Jr., Knopman DS, Weigand SD, et al. An operational approach to National Institute on AgingAlzheimer's Association criteria for preclinical Alzheimer disease. Annals of neurology 2012;71:765-775.

28. Lewczuk P, Beck G, Ganslandt 0, et al. International quality control survey of neurochemical dementia diagnostics. Neuroscience letters 2006;409:1-4.

29. Verwey NA, van der Flier WM, Blennow K, et al. A worldwide multicentre comparison of assays for cerebrospinal fluid biomarkers in Alzheimer's disease. Annals of clinical biochemistry 2009;46:235-240.

30. Mattsson N, Andreasson U, Persson S, et al. The Alzheimer's Association external quality control program for cerebrospinal fluid biomarkers. Alzheimer's \& dementia : the journal of the Alzheimer's Association 2011;7:386-395 e386.

31. Dumurgier J, Vercruysse O, Paquet C, et al. Intersite variability of CSF Alzheimer's disease biomarkers in clinical setting. Alzheimer's \& dementia : the journal of the Alzheimer's Association 2013;9:406-413.

32. Mattsson N, Andreasson U, Persson S, et al. CSF biomarker variability in the Alzheimer's Association quality control program. Alzheimer's \& dementia : the journal of the Alzheimer's Association 2013;9:251-261.

33. Hort J, Bartos A, Pirttila T, Scheltens P. Use of cerebrospinal fluid biomarkers in diagnosis of dementia across Europe. European journal of neurology : the official journal of the European Federation of Neurological Societies 2010;17:90-96. 
34. Boccardi M, Ganzola R, Bocchetta M, et al. Survey of protocols for the manual segmentation of the hippocampus: preparatory steps towards a joint EADC-ADNI harmonized protocol. Journal of Alzheimer's disease : JAD 2011;26 Suppl 3:61-75.

35. Jack CR, Jr., Barkhof F, Bernstein MA, et al. Steps to standardization and validation of hippocampal volumetry as a biomarker in clinical trials and diagnostic criterion for Alzheimer's disease. Alzheimer's \& dementia : the journal of the Alzheimer's Association 2011;7:474-485 e474.

36. Hardy JA, Higgins GA. Alzheimer's disease: the amyloid cascade hypothesis. Science 1992;256:184185.

37. Hardy J, Selkoe DJ. The amyloid hypothesis of Alzheimer's disease: progress and problems on the road to therapeutics. Science 2002;297:353-356.

38. Jack CR, Jr., Knopman DS, Jagust WJ, et al. Hypothetical model of dynamic biomarkers of the Alzheimer's pathological cascade. Lancet neurology 2010;9:119-128.

39. Jack CR, Jr., Knopman DS, Jagust WJ, et al. Tracking pathophysiological processes in Alzheimer's disease: an updated hypothetical model of dynamic biomarkers. Lancet neurology 2013;12:207216.

40. Petersen RC, Aisen P, Boeve BF, et al. Criteria for mild cognitive impairment due to alzheimer's disease in the community. Annals of neurology 2013.

41. Morgan C, Colombres M, Nunez MT, Inestrosa NC. Structure and function of amyloid in Alzheimer's disease. Progress in neurobiology 2004;74:323-349.

42. Stern Y. Cognitive reserve in ageing and Alzheimer's disease. Lancet neurology 2012;11:1006-1012.

43. Jicha GA, Parisi JE, Dickson DW, et al. Neuropathologic outcome of mild cognitive impairment following progression to clinical dementia. Archives of neurology 2006;63:674-681.

44. Salmon DP, Ferris SH, Thomas RG, et al. Age and apolipoprotein E genotype influence rate of cognitive decline in nondemented elderly. Neuropsychology 2013;27:391-401.

45. Vemuri P, Wiste HJ, Weigand SD, et al. Effect of apolipoprotein E on biomarkers of amyloid load and neuronal pathology in Alzheimer disease. Annals of neurology 2010;67:308-316.

46. Ferri CP, Prince M, Brayne C, et al. Global prevalence of dementia: a Delphi consensus study. Lancet 2005;366:2112-2117.

47. Hampel H. Current insights into the pathophysiology of Alzheimer's disease: selecting targets for early therapeutic intervention. International Psychogeriatrics 2012;24:S10-S17.

48. Fox NC, Cousens S, Scahill R, Harvey RJ, Rossor MN. Using serial registered brain magnetic resonance imaging to measure disease progression in Alzheimer disease - Power calculations and estimates of sample size to detect treatment effects. Archives of neurology 2000;57:339-344.

49. Fox NC, Black RS, Gilman S, et al. Effects of Abeta immunization (AN1792) on MRI measures of cerebral volume in Alzheimer disease. Neurology 2005;64:1563-1572.

50. Reiman EM, Caselli RJ, Chen K, Alexander GE, Bandy D, Frost J. Declining brain activity in cognitively normal apolipoprotein E epsilon 4 heterozygotes: A foundation for using positron emission tomography to efficiently test treatments to prevent Alzheimer's disease. Proceedings of the National Academy of Sciences of the United States of America 2001;98:3334-3339.

51. Jack CR, Lowe VJ, Weigand SD, et al. Serial PIB and MRI in normal, mild cognitive impairment and Alzheimers disease: implications for sequence of pathological events in Alzheimers disease. Brain 2009;132:1355-1365.

52. Rinne JO, Brooks DJ, Rossor MN, et al. 11C-PiB PET assessment of change in fibrillar amyloid-beta load in patients with Alzheimer's disease treated with bapineuzumab: a phase 2, double-blind, placebo-controlled, ascending-dose study. Lancet neurology 2010;9:363-372. 
53. Hort J, O'Brien JT, Gainotti G, et al. EFNS guidelines for the diagnosis and management of Alzheimer's disease. European journal of neurology : the official journal of the European Federation of Neurological Societies 2010;17:1236-1248.

54. Jack CR, Jr., Albert MS, Knopman DS, et al. Introduction to the recommendations from the National Institute on Aging-Alzheimer's Association workgroups on diagnostic guidelines for Alzheimer's disease. Alzheimer's \& dementia : the journal of the Alzheimer's Association 2011;7:257-262.

55. Anand R, Gill KD, Mahdi AA. Therapeutics of Alzheimer's disease: Past, present and future. Neuropharmacology 2013.

56. Savva GM, Wharton SB, Ince PG, Forster G, Matthews FE, Brayne C. Age, neuropathology, and dementia. The New England journal of medicine 2009;360:2302-2309.

57. Morbelli S, Perneczky R, Drzezga A, et al. Metabolic networks underlying cognitive reserve in prodromal Alzheimer disease: a European Alzheimer disease consortium project. Journal of nuclear medicine : official publication, Society of Nuclear Medicine 2013;54:894-902.

58. Schneider JA, Arvanitakis Z, Leurgans SE, Bennett DA. The neuropathology of probable Alzheimer disease and mild cognitive impairment. Annals of neurology 2009;66:200-208.

59. Snowdon DA, Greiner LH, Mortimer JA, Riley KP, Greiner PA, Markesbery WR. Brain infarction and the clinical expression of Alzheimer disease. The Nun Study. JAMA : the journal of the American Medical Association 1997;277:813-817.

60. Esiri MM, Nagy Z, Smith MZ, Barnetson L, Smith AD. Cerebrovascular disease and threshold for dementia in the early stages of Alzheimer's disease. Lancet 1999;354:919-920.

61. James BD, Bennett DA, Boyle PA, Leurgans S, Schneider JA. Dementia from Alzheimer disease and mixed pathologies in the oldest old. JAMA : the journal of the American Medical Association 2012;307:1798-1800.

62. Bazenet C, Lovestone S. Plasma biomarkers for Alzheimer's disease: much needed but tough to find. Biomarkers in medicine 2012;6:441-454.

63. Fagan AM, Mintun MA, Shah AR, et al. Cerebrospinal fluid tau and ptau(181) increase with cortical amyloid deposition in cognitively normal individuals: implications for future clinical trials of Alzheimer's disease. EMBO molecular medicine 2009;1:371-380.

64. Morris JC, Roe CM, Xiong C, et al. APOE predicts amyloid-beta but not tau Alzheimer pathology in cognitively normal aging. Annals of neurology 2010;67:122-131.

65. Harding AJ, Halliday GM. Cortical Lewy body pathology in the diagnosis of dementia. Acta neuropathologica 2001;102:355-363.

66. Kalaria RN, Ballard C. Overlap between pathology of Alzheimer disease and vascular dementia. Alzheimer disease and associated disorders 1999;13 Suppl 3:S115-123.

67. Visser PJ, Wolf H, Frisoni G, Gertz HJ. Disclosure of Alzheimer's disease biomarker status in subjects with mild cognitive impairment. Biomarkers in medicine 2012;6:365-368. 


\section{SUMMARY}

The advances in $\mathrm{AD}$ biomarker research over the last years have improved our understanding of the early course of $A D$ and allow us now to identify subjects with $A D$ before they are demented, which opens new opportunities for AD treatment and prevention. At present, it is still unclear how biomarkers or biomarker combinations should be used for diagnosis and prognosis of AD for clinical and research purposes. In this thesis, we studied the role of biomarkers for diagnosis and prognosis of Alzheimer's disease in cognitively normal subjects, subjects with amnestic MCI (aMCI), and subjects with non-amnestic MCI (naMCI). In part I, we described the diagnostic and prognostic ability of single and combined AD biomarkers in subjects with MCI. In part II, we focused on the recently proposed diagnostic criteria for preclinical and prodromal AD. These criteria highlight the use of biomarkers to increase the certainty of whether a subject has underlying AD pathology or not.

In Chapter 2, we provided a review and meta-analysis of the use of CSF and plasma AD biomarkers for the prediction of AD-type dementia in subjects with MCI. We showed that the combination of A $\beta 1-42$ and tau in CSF had a better predictive accuracy for AD-type dementia than single CSF $A \beta 1-42$, t-tau or p-tau markers. Plasma levels of $A \beta 1-40$ tended to predict progression to $A D$-type dementia, while plasma levels of $A \beta 1-42$, the ratio $A \beta 1$ 42/A $\beta 1-40$ and homocysteine did not predict outcome. Furthermore, in a fictive trial design with drugs that aim to prevent the progression from MCI to AD-type dementia, use of the combination of CSF A $\beta 1-42$ and tau markers in the selection of subjects could substantially reduce the sample size and trial costs.

In Chapter 3, we compared the predictive ability of the CSF A $\beta 1-42 /$ tau ratio and HCV on MRI for AD-type dementia in subjects with MCI of the European multi-center memoryclinic-based DESCRIPA study and determined the optimal test sequence of these biomarkers. While both markers were good predictors for progression to AD-type dementia and cognitive decline in subjects with $\mathrm{MCI}$, we showed that the CSF A $\beta 1-42 /$ tau ratio was the best predictor for progression. Test sequence analyses demonstrated that HCV could increase the predictive accuracy of the CSF $A \beta 1-42 /$ tau ratio. This increase in predictive accuracy for AD-type dementia and cognitive decline was because HCV predicted AD-type dementia and cognitive decline in subjects with a normal CSF A $\beta 1-42 /$ tau ratio.

In Chapter 4, the predictive accuracy of CSF A $\beta 1-42$, CSF tau, CSF A $\beta 1-42 /$ tau, HCV on MRI, and APOE genotype for AD-type dementia was compared in subjects with aMCI and naMCI of the DESCRIPA study. We found that the overall predictive accuracy for AD-type dementia was similar for subjects with aMCI and naMCI, although sensitivity of CSF and MRI markers was higher and specificity lower in subjects with aMCI. Biomarkers also predicted decline on the MMSE in both MCI groups and decline in memory and overall cognition in subjects with naMCI. While there are differences in the relationship of biomarkers to outcome in aMCI and naMCI, our findings suggest that the symptomatic manifestations of 
the $\mathrm{AD}$ pathophysiological process is broader than the amnestic presentation at the $\mathrm{MCI}$ stage and also includes non-amnestic MCI.

In Chapter 5, we assessed the progression rate to AD-type dementia and identified prognostic markers in subjects with MCI and evidence of abnormal amyloid- $\beta$ in CSF from the DESCRIPA study. In these subjects with prodromal $\mathrm{AD} / \mathrm{MCI}$ due to $\mathrm{AD}$, we found that $57 \%$ of the subjects progressed to AD-type dementia after a mean follow up of 2.2 years. High CSF levels of t-tau and p-tau and hippocampal atrophy predicted progression to ADtype dementia and decline in MMSE score. Our results implicate that markers of AD-related neuronal injury could help to identify those subjects with $\mathrm{MCI}$ due to AD that will more rapidly progress to dementia.

In Chapter 6, we investigated the influence of CSF intralaboratory and interlaboratory variability on diagnostic CSF-based AD classification in a memory-clinic population of the Dutch multi-center LeARN study. We observed clear variability in CSF AD biomarker levels between repeated analyses in the same laboratory as well as between two different laboratories, in particular for A 1-42 and p-tau. A substantial part of this variability could be explained by lot-to-lot variation of analytical kits. Our findings showed that the variability frequently led to a change in CSF-based AD diagnosis when predefined cut-offs for abnormal CSF values were applied, which may have a large impact on treatment decisions in clinical settings. Together, our results indicate that we should be careful when interpreting CSF findings and always interpret them within a clinical context.

In Chapter 7, we compared the theoretical framework of the International Working Group (IWG) criteria and National Institute on Aging-Alzheimer's Association (NIA-AA) criteria for Alzheimer's disease. The criteria revealed differences in approach, terminology and use of cognitive markers and biomarkers. Most persons who meet the IWG criteria will also meet the NIA-AA criteria and vice versa. However, the NIA-AA criteria allow for a subclassification of persons based on biomarker results within each diagnostic category. Validation and likely also modification of these criteria are needed before they can be applied in clinical practice.

In Chapter 8, we described the prevalence and prognosis of subjects with preclinical $\mathrm{AD}$ according to CSF biomarkers, episodic memory and the proposed NIA-AA criteria. Subjects were recruited from longitudinal studies of memory and aging in cognitively normal community-dwelling volunteers at the Knight Alzheimer's Disease Research Center (ADRC) of the Washington University School of Medicine in St. Louis (USA). We showed that preclinical AD can be defined by CSF markers, is common in individuals aged at least 65 years, and is associated with an increased risk of cognitive decline, progression to CDR at least 0.5 symptomatic AD, and mortality. Progression rates differed between the preclinical AD stages; thus, stages 1,2 , and 3 likely represent different and progressive disease severities. Hence, preclinical AD could be an important target for therapeutic intervention.

In Chapter 9, the prevalence and prognosis of prodromal AD was examined based on well-established biomarkers and the proposed IWG and NIA-AA criteria. Subjects were recruited from multiple centers of the European Alzheimer's Disease Consortium (EADC) 
and the Alzheimer's Disease Neuroimaging Initiative (ADNI). Our findings showed that subject classifications based on the IWG and NIA-AA criteria were both good predictors of likelihood of future cognitive decline and progression to AD-type dementia in subjects with MCI. However, our results indicated that knowledge about both amyloid pathology and neuronal injury is preferred to give an optimal prognosis. Moreover, the use of multiple neuronal injury markers in combination with an amyloid marker can help to identify the subjects that will soon progress to AD-type dementia. Also, a more lenient CSF A $\beta 1-42$ cutoff may be needed to identify subjects with SNAP who have prodromal AD.

In Chapter 10, we provided a general discussion of our findings, its implications for research, AD trials and clinical practice, and recommendations for future research. Furthermore, this last chapter includes a summary of the findings, list of author affiliations, list of publications, information about the author, and acknowledgements. 



\section{SAMENVATTING}

De vooruitgang de afgelopen jaren in biomarker onderzoek bij de ziekte van Alzheimer (AD) heeft ons begrip van de vroege fase van $A D$ verbeterd en staat ons toe om nu mensen te identificeren met $\mathrm{AD}$ vooraleer ze dement zijn. Dit zorgt voor nieuwe mogelijkheden voor behandeling en preventie van AD. Op dit moment is het nog steeds onduidelijk hoe biomarkers of biomarker combinaties gebruikt moeten worden voor de diagnose en prognose van $\mathrm{AD}$, zowel voor klinische als wetenschappelijke doeleinden. In dit proefschrift hebben we de rol van biomarkers voor de diagnose en prognose van AD bestudeerd bij cognitief normale mensen, mensen met milde amnestische stoornissen en mensen met milde non-amnestische stoornissen. In deel I hebben we de diagnostische en prognostische waarde van aparte of gecombineerde biomarkers voor AD bij mensen met milde cognitive stoornissen (MCI) beschreven. In deel II hebben we ons gericht op de recent voorgestelde diagnostische criteria voor preklinische en prodromale AD. Deze criteria benadrukken het gebruik van biomarkers om meer zekerheid te krijgen of er al dan niet sprake is van onderliggende $\mathrm{AD}$ pathologie.

In Hoofdstuk 2 hebben we een overzicht en meta-analyse gepresenteerd van het gebruik van $\mathrm{AD}$ biomarkers in hersenvocht (CSF) en plasma. We toonden aan dat de combinatie van $A \beta 1-42$ en tau in CSF een betere voorspeller was voor AD-type dementie dan $A \beta 1-42$, t-tau of p-tau apart. $A \beta 1-40$ in plasma toonde een tendens om progressie naar $A D$-type dementie te voorspellen, terwijl plasma waardes van $A \beta 1-42$, de ratio $A \beta 1-42 / A \beta 1$ 40 , en homocysteïne de uitkomst niet voorspelden. In een fictief trial design met medicatie om progressie van $\mathrm{MCI}$ naar AD-type dementia te voorkomen vonden we dat het gebruik van de combinatie van CSF A $\beta 1-42$ en tau markers voor de selectie van mensen het aantal mensen nodig voor de trial evenals de kosten voor de trial aanzienelijk kon verminderen.

In Hoofdstuk 3 hebben we de voorspellende waarde van de CSF A $\beta 1-42 /$ tau ratio en hippocampus volume (HCV) vergeleken voor progressie naar AD-type dementie bij mensen met MCI binnen de Europese DESCRIPA studie waaraan meerdere Europese centra (geheugenpoliklinieken) deelnamen. Daarnaast hebben we de optimale testvolgorde van deze biomarkers bepaald. Ondanks dat beide markers goede voorspellers waren voor progressie naar $\mathrm{AD}$-type dementie en cognitieve achteruitgang vonden we dat de CSF A $\beta 1$ $42 /$ tau ratio de beste voorspeller was voor progressie. Analyses voor testvolgorde toonden aan dat HCV de voorspellende waarde van de CSF A $\beta 1-42 /$ tau ratio kon doen toenemen. Deze toename in voorspellende accuraatheid voor AD-type dementie en cognitive achteruitgang was er doordat HCV progressie voorspelde bij mensen met een normale CSF A $\beta 1-42 /$ tau ratio.

In Hoofdstuk 4 werd de voorspellende accuraatheid van CSF A $\beta 1-42$, CSF tau, CSF A $\beta 1-42 /$ tau, HCV, en APOE genotype voor AD-type dementie vergeleken bij mensen met amnestische MCI (aMCI) en non-amnestische MCI (naMCI). We vonden dat de algemene voorspellende waarde voor AD-type dementie vergelijkend was bij mensen met aMCI en naMCI, hoewel de sensitiviteit van CSF en MRI markers hoger was en de specificiteit lager 
was bij mensen met aMCI. Biomarkers voorspelden ook achteruitgang op de MMSE in beide MCI groepen en achteruitgang in geheugen en algemene cognitie bij mensen met naMCI. Ondanks dat er verschillen waren in de relatie tussen biomarkers en uitkomst bij aMCI en naMCI tonen onze resultaten aan dat de symptomatische manifestaties van het AD pathofysiologisch proces breeder zijn dan de amnestische presentatie in de MCI fase en ook non-amnestische MCI bevat.

In Hoofdstuk 5 onderzochten we het aantal mensen dat AD-type dementie ontwikkelde en identificeerden we prognostische markers bij mensen met MCI en abnormaal amyloid- $\beta$ in CSF van de DESCRIPA study. Bij deze mensen met prodromale $\mathrm{AD} / \mathrm{MCI}$ als gevolg van $\mathrm{AD}$ vonden we dat $57 \% \mathrm{AD}$-type dementie kreeg na een gemiddelde follow-up van 2.2 jaar. Hoge CSF waardes van t-tau en p-tau en hippocampus atrofie voorspelden progressie naar AD-type dementie en achteruitgang op de MMSE. Onze resultaten impliceren dat markers voor AD-gerelateerde neuronale schade zouden kunnen helpen om mensen met MCI als gevolg van AD te identificeren wat sneller AD-type dementie zullen krijgen.

In Hoofdstuk 6 onderzochten we de invloed van CSF variabiliteit tussen en binnen laboratoria op de diagnostische classificatie van AD gebaseerd op CSF data in een geheugenpolipopulatie van de Nederlandse LeARN studie waaraan meerdere centra deelnamen. We observeerden duidelijke variabiliteit in CSF AD biomarker waardes tussen herhaalde metingen in hetzelfde laboratorium evenals tussen twee verschillende laboratoria, in het bijzonder voor $A \beta 1-42$ en p-tau. Een substantieel gedeelte van deze variabiliteit zou kunnen verklaard worden door variatie tussen lots van analytische kits. Onze bevindingen toonden aan dat de variabiliteit vaak leidde tot verandering van AD diagnose wanneer vooraf gedefineerde afkappunten gebruikt werden om abnormale CSF waardes te bepalen, hetgeen een grote impact kan hebben op behandelbeslissingen in klinische settings. Algeheel tonen onze resultaten aan dat we voorzichtig moeten zijn bij het interpreteren we CSF waardes en dat we CSF waardes altijd in een klinische context moeten interpreteren.

In Hoofdstuk 7 vergeleken we het theoretische kader van de International Working Group (IWG) criteria en de National Institute on Aging-Alzheimer's Association (NIA-AA) criteria. Deze criteria tonen verschillen in aanpak, terminologie en gebruik van cognitieve markers en biomarkers. De meeste mensen wat aan de IWG criteria voldoen zullen ook aan de NIA-AA criteria voldoen en andersom. Hoe dan ook, de NIA-AA criteria staan subclassificatie van mensen toe gebaseerd op biomarker resultaten binnen elke diagnostische categorie. Validatie en wellicht ook aanpassingen van deze criteria zijn nodig alvorens deze toegepast kunnen worden in de klinische praktijk.

In Hoofdstuk 8 beschreven we de prevalentie en prognose van mensen met preklinische AD gebaseerd op CSF biomarkers, episodisch geheugen, en de voorgestelde NIA-AA criteria. Deelnemers werden gerecruteerd van longitudinale studies naar geheugen en veroudering bij cognitief normale vrijwilligers uit de samenleving aan het Knight Alzheimer's Disease Research Center (ADRC) van Washington University School of Medicine 
in St. Louis (VS). We toonden aan dat preklinische AD gemeten kan worden met CSF markers, veel voorkomt bij mensen van 65 jaar en ouder, en geassocieerd is met een verhoogd risico op cognitieve achteruitgang, progressie naar een CDR van 0.5 of groter met symptomatische AD, en mortaliteit. Progressie verschilde tussen de preklinische AD fases; dus fase 1, 2, en 3 representeren wellicht een verschillende en progressieve ziekte-ernst. Dit geeft aan dat preklinische $\mathrm{AD}$ een belangrijk doelwit kan zijn voor therapeutische interventie.

In Hoofdstuk 9 onderzochten we de prevalentie en prognose van prodromale AD door het gebruik van erkende biomarkers en de voorgestelde IWG en NIA-AA criteria. Mensen werden geworven van verschillende centra van het European Alzheimer's Disease Consortium (EADC) en Alzheimer's Disease Neuroimaging Initiative (ADNI). Onze bevindingen toonden aan dat classificaties van individuen gebaseerd op de IWG en NIA-AA criteria goede voorspellers waren voor de kans op cognitieve achteruitgang of progressie naar AD-type dementie in de toekomst. Desalniettemin toonden onze resultaten aan dat kennis over zowel amyloïde pathologie als neuronale schade de voorkeur heeft om een optimale prognose te geven. Ook kan het gebruik van meerdere neuronale schade markers in combinatie met een amylö̈de marker helpen om de mensen te identificeren wat sneller AD-type dementie zullen ontwikkelen. Daarnaast kan een liberaler CSF A $\beta 1-42$ afkappunt nodig zijn om de mensen met enkel neuronale schade en prodromale AD (SNAP) te identificeren .

Hoofdstuk 10 bevat een algemene discussie van onze bevindingen, de implicaties hiervan voor onderzoek en kliniek en aanbevelingen voor verder onderzoek. Dit laastse hoofdstuk bevat tevens een samenvatting van de resultaten, overzicht van auteursaffiliaties, lijst van publicaties, informatie over de auteur en dankwoord. 



\section{AUTHOR AFFILIATIONS}

Pauline Aalten, $P h D$

Department of Psychiatry and Neuropsychology, Maastricht University, School for Mental Health and Neuroscience, Alzheimer Center Limburg, Maastricht, the Netherlands

Frederik Barkhof, $M D, P h D$

Department of Radiology, VU University Medical Center, Amsterdam, the Netherlands

Christine Bastin, $\mathrm{PhD}$

Cyclotron Research Centre, University of Liège, Belgium

Bart N.M. van Berckel, MD, PhD

Department of Nuclear Medicine and PET Research, VU University Medical Center, Amsterdam, The Netherlands

Kaj Blennow, $M D, P h D$

Clinical Neurochemistry Laboratory, Göteborg University, Sahlgrenska University Hospital, Mölndal, Sweden

Leah Burns, MPH

Global Health Economics \& Outcomes Research, Bristol-Myers Squibb Company, Wallingford, Connecticut, USA

Nigel J. Cairns, $P h D$

Department of Neurology, Department of Pathology and Immunology, The Knight Alzheimer's Disease Research Center, Washington University School of Medicine, St. Louis, Missouri, USA

Mira Didic, $M D P h D$

Service de Neurologie and Neuropsychologie, Pôle de neurosciences cliniques, AP-HM Timone, INSERM U751, Université de la Méditerranée, Marseille, France

Sebastiaan Engelborghs, $M D, P h D$

Reference Center for Biological Markers of Dementia (BIODEM), Laboratory of Neurochemistry and Behavior, Institute Born-Bunge, University of Antwerp, Antwerp, Belgium; and Department of Neurology and Memory Clinic, Hospital Network Antwerp, Middelheim and Hoge Beuken, Antwerp, Belgium 


\section{Anne M. Fagan, $P h D$}

Department of Neurology, Washington University School of Medicine, The Knight Alzheimer's Disease Research Center, St. Louis, Missouri, USA.

\section{Giovanni B. Frisoni, MD}

IRCCS San Giovanni di Dio Fatebenefratelli, Laboratory of Epidemiology and Neuroimaging and Translational Research Unit, Brescia, Italy

\section{Lutz Froelich, $M D, P h D$}

Department of Geriatric Psychiatry, Zentralinstitut für Seelische Gesundheit, University of Heidelberg, Mannheim, Germany

Wiesje van der Flier, $P h D$

Alzheimer center \& Department of Neurology, Neuroscience Campus Amsterdam, VU University Medical Center, Amsterdam, The Netherlands

\section{Elizabeth A. Grant, PhD}

Division of Biostatistics, The Knight Alzheimer's Disease Research Center, Washington University School of Medicine, St. Louis, Missouri, USA

\section{Harald Hampel, MD, PhD}

Department of Psychiatry, Psychosomatic Medicine and Psychotherapy, Goethe-University, Frankfurt, Germany

Ron Handels, MSc

Department of Psychiatry and Neuropsychology, Maastricht University, School for Mental Health and Neuroscience, Alzheimer Center Limburg, Maastricht, the Netherlands

\section{Jason Hassenstab, PhD}

Department of Psychology, The Knight Alzheimer's Disease Research Center, Washington University School of Medicine, St. Louis, Missouri, USA

Sanna-Kaisa Herukka, MD, PhD

Department of Neurology, School of Medicine, University of Eastern Finland and Kuopio University Hospital, Kuopio, Finland

David M. Holtzman, MD, PhD

Department of Neurology, The Knight Alzheimer's Disease Research Center, Hope Center for Neurological Disorders, Washington University School of Medicine, St. Louis, Missouri, USA 
Mateusz S. Jasielec, MS

Division of Biostatistics, Washington University School of Medicine, St. Louis, Missouri, USA

Dirk L. Knol, PhD

Department of Epidemiology and Biostatistics, VU University Medical Center, Amsterdam, the Netherlands

Raquel Lemos, $P h D$

Faculty of Psychology and Educational Sciences, University of Coimbra, Coimbra, Portugal

Gilbert L'Italien, PhD

Global Health Economics \& Outcomes Research, Bristol-Myers Squibb Company, Wallingford, Connecticut, USA

Michaela Litchmore-Dunbar, BSc

Department of Education Support, King's College London, Institute of Psychiatry, London, UK

Alexandre de Mendonça, MD

Neurological Clinical Research Unit, Instituto de Medicina Molecular, Lisboa, Portugal

Lennart Minthon, $M D, P h D$

Clinical Memory Research Unit, Department of Clinical Sciences Malmö, Lund University, Malmö, Sweden

Silvia Morbelli, $M D, P h D$

Nuclear Medicine, Department of Internal Medicine, University of Genoa, Genoa, Italy

John C. Morris, $M D, P h D$

Department of Neurology, Department of Pathology and Immunology, The Knight Alzheimer's Disease Research Center, Washington University School of Medicine, St. Louis, Missouri, USA

Cristina Muscio, PhD

IRCCS San Giovanni di Dio Fatebenefratelli, Laboratory of Epidemiology, Neuroimaging and telemedicine, Brescia, Italy

Flavio Nobili, $M D$

Clinical Neurophysiology Service, Department of Neurosciences, Ophthalmology and Genetics, University of Genoa, Genoa, Italy 
Marcel G.M. Olde Rikkert, $M D, P h D$

Department of Geriatric Medicine, Radboud University Nijmegen Medical Centre, Nijmegen, The Netherlands; and Donders Institute for Brain, Cognition and Behaviour, Radboud University Nijmegen and Radboud Alzheimer Centre, Nijmegen, The Netherlands

Robert Perneczky, MD, PhD

Department of Psychiatry and Psychotherapy, Technische Universität München, Munich, Germany

Inez Ramakers, $P h D$

Department of Psychiatry and Neuropsychology, Maastricht University, School for Mental Health and Neuroscience, Alzheimer Center Limburg, Maastricht, the Netherlands

Ineke A. van Rossum, $M D, P h D$

Alzheimer center \& Department of Neurology, Neuroscience Campus Amsterdam, VU University Medical Center, Amsterdam, The Netherlands

Daniel Rueckert, PhD

Department of Computing, Imperial College London, London, UK

Erik Salmon, $M D, P h D$

Memory Clinic, Department of Neurology, CHU Liège, Belgium; and Cyclotron Research Centre, Liège, Belgium

Isabel Santana, $M D, P h D$

Department of Neurology, Coimbra University Hospital, Coimbra, Portugal

Philip Scheltens, $M D, P h D$

Alzheimer center \& Department of Neurology, Neuroscience Campus Amsterdam, VU University Medical Center, Amsterdam, The Netherlands

Dina Silva, PhD

Dementia Clinics, Faculty of Medicine, University of Lisbon, Lisbon, Portugal; and Instituto de Farmacologia e Neurociências, Edifício Egas Moniz, Faculdade de Medicina de Lisboa, Lisboa, Portugal

Andy Simmons, PhD

Department of Clinical Neuroscience, Centre for Neuroimaging Science, King's College London, Institute of Psychiatry, London, UK 
Hilkka Soininen, $M D, P h D$

Department of Neurology, University and University Hospital of Kuopio, Kuopio, Finland

Charlotte E. Teunissen, $P h D$

Department of Clinical Chemistry, VU University Medical Center, Amsterdam, the Netherlands

Magda Tsolaki, $M D, P h D$

Aristotle University of Thessaloniki, Memory and Dementia Center, 3rd Department of Neurology, “G Papanicolaou” General Hospital, Thessaloniki, Greece

Stefan Van der Mussele, MSc

Reference Center for Biological Markers of Dementia (BIODEM), Laboratory of Neurochemistry and Behavior, Institute Born-Bunge, University of Antwerp, Antwerp, Belgium; and Department of Nursing and Midwifery Sciences, Faculty of Medicine and Health Sciences, University of Antwerp, Antwerp, Belgium

Marcel M. Verbeek, PhD

Department of Neurology and Department of Laboratory Medicine, Radboud University Nijmegen Medical Centre, Nijmegen, The Netherlands; and Donders Institute for Brain, Cognition and Behaviour, Radboud University Nijmegen and Radboud Alzheimer Centre, Nijmegen, The Netherlands

Frans R.J. Verhey, MD, PhD

Department of Psychiatry and Neuropsychology, Maastricht University, School for Mental Health and Neuroscience, Alzheimer Center Limburg, Maastricht, the Netherlands

Pieter Jelle Visser, $M D, P h D$

Department of Psychiatry and Neuropsychology, Maastricht University, School for Mental Health and Neuroscience, Alzheimer Center Limburg, Maastricht, the Netherlands; and Alzheimer center \& Department of Neurology, Neuroscience Campus Amsterdam, VU University Medical Center, Amsterdam, The Netherlands

Lars-Olof Wahlund, $M D, P h D$

Department of NVS, Section of Clinical Geriatrics, Karolinska Institutet, Karolinska University Hospital, Huddinge, Sweden

Robin Wolz, PhD

Department of Computing, Imperial College London, London, UK 
Chengjie Xiong, PhD

Division of Biostatistics, The Knight Alzheimer's Disease Research Center, Washington University School of Medicine, St. Louis, Missouri, USA 


\section{PUBLICATIONS}

\section{International peer reviewed journals}

Vos SJB, Xiong C, Visser PJ, Jasielec MS, Hassenstab J, Grant EA, Cairns NJ, Morris JC, Holtzman DM, Fagan AM. Preclinical Alzheimer's disease and its outcome: a longitudinal cohort study. The Lancet Neurology 2013;12(10):957-65.

Vos SJB, van Rossum IA, Verhey F, Knol DL, Soininen H, Wahlund L-O, Hampel H, Tsolaki M, Minthon L, Frisoni GB, Froelich L, Nobili F, van der Flier W, Blennow K, Wolz R, Scheltens P, Visser PJ. Prediction of Alzheimer's disease in subjects with amnestic and non-amnestic MCI. Neurology 2013; 80(12):1124-1132.

Visser PJ, Vos S, van Rossum I, Scheltens P. Comparison of International Working Group criteria and National Institute on Aging-Alzheimer's Association criteria for Alzheimer's disease. Alzheimer's \& Dementia 2012;8:560-563.

van Rossum IA, Vos SJB, Burns MPH, Knol DL, Scheltens P, Soininen H, Wahlund L-O, Hampel H, Tsolaki M, Minthon L, L'Italien G, van der Flier WM, Teunissen CE, Blennow K, Barkhof F, Rueckert D, Wolz R, Verhey F, Visser PJ. Injury markers predict cognitive decline in subjects with MCI and amyloid pathology. Neurology 2012;79:1809-1816.

Vos S, van Rossum I, Burns L, Knol D, Scheltens P, Soininen H, Wahlund L-O, Hampel H, Tsolaki M, Minthon L, Handels R, L'Italien G, van der Flier W, Aalten P, Teunissen C, Barkhof F, Blennow K, Wolz R, Rueckert D, Verhey F, Visser PJ. Test sequence of CSF and MRI biomarkers for prediction of AD in subjects with MCI. Neurobiology of Aging 2012;33:22722281.

Van Rossum I, Vos S, Handels R, Visser PJ. Biomarkers as predictor for conversion from MCI to Alzheimer-type dementia. Implications for trial design. Journal of Alzheimer's Disease 2010;20:881-891.

Vos SJB, Visser PJ, Verhey F, Aalten P, Ramakers I, Scheltens P, Olde-Rikkert MGM, Verbeek MM, Teunissen CE. Variability of CSF Alzheimer's disease biomarkers: implications for clinical practice. Submitted.

\section{National peer reviewed journals}

Vos SJ, Visser PJ, \& Verhey FRJ. The role of CSF biomarkers in the early diagnosis of Alzheimer's disease. Tijdschrift voor Psychiatrie 2011;53(9): 647-653. 


\section{Abstracts}

Vos SJB, Xiong C, Grant EA, Visser PJ, Cairns NJ, Morris JC, Holtzman DM, Fagan AM. Using CSF markers to identify stages of NIA-AA preclinical Alzheimer's disease and its prognosis. Alzheimer's Association International Conference (AAIC) 2013, Boston (USA).

Vos SJB, van Rossum I, Verhey F, Soininen H, Wahlund L-O, Hampel H, Tsolaki M, Minthon L, van der Flier W, Teunissen C, Blennow K, Scheltens P, Visser PJ. CSF markers for predicting Alzheimer-type dementia in subjects with amnestic versus non-amnestic mild cognitive impairment. Alzheimer's Association International Conference (AAIC) 2012, Vancouver (Canada).

Vos SJB, Verhey F, Scheltens P, Soininen H, Wahlund L-O, Hampel H, Tsolaki M, Minthon L, Frisoni G, Frolich L, Nobili F, van der Flier W, Blennow K, Wolz R, Visser PJ. Biomarkers for prediction of AD-type dementia in subjects with amnestic versus non-amnestic MCI. International Psychogeriatric Association (IPA) 2011, den Haag (the Netherlands).

Vos SJB, Burns L, Scheltens P, Soininen H, Wahlund L-O, Hampel H, Tsolaki M, Minthon L, Verhey F, L'Italien G, van der Flier W, Teunissen C, Blennow K, Wolz R, Visser PJ. CSF and MRI biomarkers as predictors for Alzheimer-type dementia in subjects with MCI. Alzheimer's Association International Conference (AAIC) 2011, Paris (France).

Vos SJB, Verhey F, Scheltens P, Soininen H, Wahlund L-O, Hampel H, Tsolaki M, Minthon L, Frisoni G, Frolich L, Nobili F, van der Flier W, Blennow K, Wolz R, Visser PJ. CSF, MRI, and APOE biomarkers as predictors for Alzheimer-type dementia in subjects with amnestic versus non-amnestic mild cognitive impairment. Alzheimer's Association International Conference (AAIC) 2011, Paris (France).

Vos SJB, Elias-Sonnenschein L, Verhey F, Visser PJ. Meta-analysis of CSF biomarkers as predictor of conversion of mild cognitive impairment to Alzheimer-type dementia. Alzheimer's Association International Conference (AAIC) 2010, Honolulu (USA).

Vos SJB, van Rossum I, Verhey F, Wahlund L-O, Tsolaki M, Minthon L, Hampel H, Bürger K, Pirttila T, Soininen H, Olde-Rikkert M, Verbeek M, Spiru L, Blennow K, van der Flier WM, Blankenstein M, Scheltens P, Visser PJ. CSF and MRI biomarkers as predictors for conversion from mild cognitive impairment to Alzheimer-type dementia. Alzheimer's Association International Conference (AAIC) 2010, Honolulu (USA). 


\section{AUTHOR INFORMATION}

Stephanie Vos is born in Tongeren on July 301986 and grew up in Riemst (Belgium). After graduating from high school at the Heilig Graf-instituut Bilzen in 2004, she started to study Psychology at Maastricht University. During her training she got selected to follow an Honors program in Psychology. In 2007, Stephanie started the Research Master Neuropsychology, for which she performed a research and clinical internship at the Department of Psychiatry and Neuropsychology of the Academical Hospital of Maastricht. In 2009, she graduated cum laude and started working as a PhD candidate at the Department of Psychiatry and Neuropsychology under supervision of Prof. dr. Frans Verhey and Dr. Pieter Jelle Visser. As part of her PhD project, she worked for 3 months at Washington University in St. Louis to collaborate with Prof. dr. Anne Fagan and colleagues. Presently, Stephanie Vos works as a postdoctoral researcher at the Department of Psychiatry and Neuropsychology.

Stephanie Vos is geboren in Tongeren op 30 juli 1986 en groeide op in Riemst (België). In 2004 rondde ze haar middelbare school af aan het Heilig Graf-instituut te Bilzen, waarna ze aan haar opleiding Psychologie begon aan de Universiteit Maastricht. Tijdens deze opleiding werd ze geselecteerd om een Honors programma in Psychologie te volgen. In 2007 begon ze aan de onderzoeksmaster Neuropsychologie en in kader hiervan deed ze een stage op de afdeling Psychiatrie en Neuropsychologie van het Academisch ziekenhuis Maastricht. In 2009 studeerde ze cum laude af en startte ze haar werk als promovendus op de afdeling Psychiatrie en Neuropsychologie van de Universiteit Maastricht onder leiding van Prof. dr. Frans Verhey en Dr. Pieter Jelle Visser. Als onderdeel van haar promotieproject heeft ze 3 maanden gewerkt aan Washington University in St. Louis om samen te werken met Prof. dr. Anne Fagan en collega's. Stephanie Vos werkt momenteel als postdoc onderzoeker op de afdeling Psychiatrie en Neuropsychologie. 



\section{DANKWOORD}

Vier jaar later en aan het einde van dit proefschrift wil ik graag even terugblikken op de afgelopen tijd en iedereen bedanken die, op welke manier dan ook, een bijdrage heeft geleverd aan het tot stand komen van dit proefschrift.

Allereest wil ik graag alle patiënten en mantelzorgers bedanken voor hun deelname aan onze onderzoeken. De klinische werkvloer was steeds een aangename afwisseling voor mij en heeft tevens voor een berg aan onderzoeksdata gezorgd.

Bijzondere dank gaat uit naar mijn promotor, Prof. dr. Verhey, and co-promotor, Dr. Visser, voor de prettige samenwerking de afgelopen jaren.

Beste Frans, ik ben blij dat je me de kans hebt gegeven om me als wetenschapper te ontwikkelen en te verdiepen in de intrigerende Alzheimer-wereld. Bedankt voor het vertrouwen en voor de getoonde belangstelling in mijn onderzoek. Jouw toegankelijkheid, betrokkenheid en klinische blik op mijn onderzoek heb ik zeer op prijs gesteld.

Beste Pieter Jelle, ik heb ontzettend veel van jou geleerd de afgelopen jaren. Bedankt voor het delen van jouw grote netwerk van contacten, de mini-colleges op het krukje, jouw aanstekelijke enthousiasme en jouw kritische kijk op mijn onderzoek. Ik waardeer dat je, ondanks je drukke agenda, zowat op elk tijdstip van de dag bereikbaar was voor mij. Ik kijk uit naar onze verdere samenwerking, evenals naar de Belgenmoppen en mijn fles wijn.

Dr. Aalten en Dr. Ramakers, bedankt voor jullie waardevolle hulp langs de zijlijn.

Beste Pauline, ik bewonder jouw management skills. Dank om het gehele overzicht en verloop van de LeARN studie strak in de gaten te houden.

Beste Inez, het is mooi om te zien hoe jij kliniek en onderzoek weet te combineren. Bedankt dat ik steeds voor alle ins en outs van de biobank en patiënten follow-up bij jou terecht kon.

Prof. dr. Fagan, dear Anne, it was great working with you and the biomarker core in St. Louis. You are a wonderful person and great scientist. Thanks for your hospitality and for making me feel home. I am glad that our work resulted in a terrific publication and I am grateful to Prof. dr. Holtzman, Prof. dr. Morris, Prof. dr. Xiong, Prof. dr. Cairns, Dr. Grant, Dr. Hassenstab, and Matt for their scientific inputs. I am looking forward to our further collaborations. Aarti, Sushi, Matt, and particularly Courtney and Jane, thanks for hanging out with me and showing me around St. Louis. It was a wonderful experience!

All members of the reading committee: Prof. dr. Ponds, Prof. dr. Jessen, Prof. dr. van Oostenbrugge, Dr. Köhler, and Dr. Schoonenboom, thank you for taking the time to read and judge my dissertation. 
All co-authors of my articles, thank you for your thoughtful comments, which provided significant contributions to the quality of this dissertation. Ineke, jij in het bijzonder bedankt voor de fijne samenwerking de afgelopen jaren.

Dikke merci aan mijn lieve paranimfen, Lies en Sanne. Lies, we zijn samen aan dit LeARN avontuur begonnen en ik had me de afgelopen 4 jaar geen betere buddy kunnen voorstellen. Fijn dat we al onze promotiefrustraties en -successen konden delen en bedankt voor de gezelligheid. Veel succes in Jülich en ik ga extra uitkijken naar de maandagen nu.

Sanne, jij bent al een goede vriendin sinds onze studie en ik ben blij dat we nu ook collega's zijn. Dank voor de fijne tijd als PhD vertegenwoordigers, jouw interesse in mijn onderzoek en de nodige afleiding hiervan. Veel succes met het afronden van je proefschrift.

Bedankt aan alle lieve collega's voor de onmiskenbare hulp en fijne sfeer op de afdeling. Ron, mijn trouwe kamergenoot, jij wist onze productiviteit te maximalizeren door onze bureau goed fris te houden en van voldoende lekkers te voorzien. Dank voor de fijne samenwerking en de vele inspirerende discussies en succes met het vaderschap. Renske, bedankt voor de belangrijke ondersteuning tijdens de piekmomenten van klinisch werk en voor de vele gezellige koffiepauzes en overheerlijke muffins. Heidi, Inez, Lies, Lyzel, Pauline, Pieter Jelle, Renske, Saartje en Willemijn: bedankt voor het aangename gezelschap tijdens de vele congressen. Astrid, dank voor de zorgvuldige planning van onze patiënten, de logistiek verliep erg soepel dankzij jou. Carla en Tanja, het was prettig om met jullie samen te werken, bedankt voor het uitvoeren van de vele mantelzorggesprekken. Suzanne, jij was er aan het begin bij om alles nauwkeurig op te starten, bedankt daarvoor. Elsa en Els, dank voor de administratieve ondersteuning wanneer nodig. Nico, bedankt voor de datainvoer, technische ondersteuning van de datasets en de oplossingen die je, samen met Ron, had voor al mijn Mac-problemen. Alle stagiaires: Susanne, Rens, Christy, Caroline, Lorraine, Iris, Anneloes, Nele, Eefje en Mijke, dank voor jullie onmisbare hulp en inzet bij de patiënten follow-up en datainvoer. Mede ex-PhD vertegenwoordigers Sanne P., Martine, Jo, Caroline, Romina, Sanne S., het was steeds een aangename afwisseling om met jullie de MHeNS AIO activiteiten te organiseren en pizza te eten.

Mijn lieve vrienden en familie wil ik bedanken voor de steun en interesse in mijn onderzoek de afgelopen jaren, evenals voor de leuke feestjes, etentjes, en uitstapjes.

Mama en papa, bedankt dat jullie er altijd voor me zijn en me steunen met alles wat ik doe. Dankzij jullie ben ik geworden wie ik ben en doe ik wat ik graag doe, merci voor alles! Véronique, jij bruist van energie en weet me steeds aan het lachen te brengen. Wij zijn altijd al 2 handen op één buik geweest; ik ben trots om je zus te zijn!

Fabien, faire un doctorat ensemble a ses avantages et désavantages. Je suis heureuse d'avoir pu toujours trouver la paix avec toi et que tu étais toujours là pour moi, même dans les moments vraiment stressant pour toi. Merci Fab, je t'aime! 

